\title{
PIERRE ROBERT
}

\section{Sur l'axiomatique des systèmes générateurs, des rangs,} Applications à certains problèmes combinatoires

\author{
Mémoires de la S. M. F., tome 14 (1968) \\ <http://www.numdam.org/item?id=MSMF_1968_14_3_0>
}

CC Mémoires de la S. M. F., 1968, tous droits réservés.

L'accès aux archives de la revue « Mémoires de la S. M. F. » (http://smf. emath.fr/Publications/Memoires/Presentation.html) implique l'accord avec les conditions générales d'utilisation (http://www.numdam.org/conditions). Toute utilisation commerciale ou impression systématique est constitutive d'une infraction pénale. Toute copie ou impression de ce fichier doit contenir la présente mention de copyright.

\section{Numdam}


Mémoire 14, 1968, $192 \mathrm{p}$.

Sur I'axiomatique des systèmes générateurs, des rangs, ...

Applications à certains problèmes combinatoires

par Pierre ROBERT (*)

Table des matières

Introduction $\ldots \ldots \ldots \ldots \ldots \ldots \ldots \ldots \ldots \ldots \ldots \ldots \ldots \ldots \ldots \ldots \ldots \ldots \ldots \ldots \ldots \ldots \ldots \ldots \ldots \ldots \ldots \ldots \ldots \ldots$

Planches I, II et III $\quad \ldots \ldots \ldots \ldots \ldots \ldots \ldots \ldots \ldots \ldots \ldots \ldots \ldots \ldots \ldots \ldots \ldots \ldots \ldots 12$

Chapitre I Axiomatique sur les parties libres et les parties ou

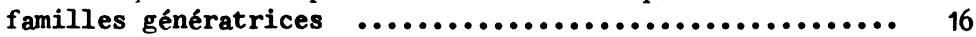

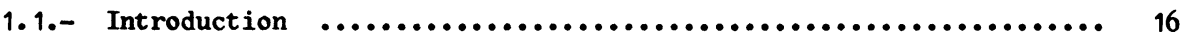

1.2.- Parties libres, rangs, parties liées, bases et ciricuits ........ 17

1.3.- Fonctions génératrices et parties libres .................... 26

1.4.- Foncteur de fermeture. Régularisation. Treillis de sous-espaces. $\varphi$-espaces $\quad$........................................ 32

Chapitre II Les structures de $\mathscr{L}$-espace et de $\varphi$-espace .......... 43

2.1.- Généralités - Comparaisons de structures .................. 43

2.2.- Structures initiales dans les $\mathscr{L}$-espaces et les $\varphi$-espaces ..... 54

2.3.- Structures finales dans les $\mathscr{L}$-espaces et les $\varphi$-espaces ....... 57

2.4.- Irréductibilités - Isomorphismes ......................... 59

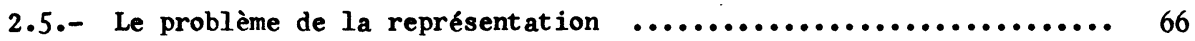

Chapitre III La théorie de la disjonction ...................... 69

3.1.- Définition et propriétés générales ...................... 70

3.2.- Les théorèmes fondamentaux sur la disjonction dans les $\varphi$-espaces.. 76

3.3.- Caractérisation des familles disjointes avec le rang ( $\varphi$-espace)... 86

3.4.- La disjonction dans une $\mathscr{L}$-algèbre $\ldots \ldots \ldots \ldots \ldots \ldots \ldots \ldots \ldots \ldots \ldots . . . . . . \ldots 0$

(*) Thèse Sci. math. Rennes, 1966 
Chapitre IV Existence de familles disjointes incluses dans une famille donnée .............................. 99

4.1.- Notations et terminologies générales ..................... 99

4.2.- Cas de familles finies de cardinaux finis ................. 100

4.3.- Application à la résolution pratique du cas fini ............ 105

4.4.- Propriétés générales des familles de type $A$. Applications ... 108

4.5.- Cas de familles finies de Cardinaux quelconques ............ 110

4.6.- Non-extension de ces résultats aux $\mathscr{L}$-espaces $\ldots \ldots \ldots \ldots \ldots \ldots \ldots .113$

Chapitre $v$ Existence de familles disjointes incluses dans des familles infinies de parties d'un $\varphi$-espace ........... 115

5.1.- Généralités ...................................... 115

5.2.- Familles $t$ de cardinaux finis ......................... 118

5.3.- Réduction de l'étude d'une famille $\left(x_{i}\right)_{i e I}$ quelconque $\ldots . . . . . .124$

5.4.- Familles $t$ de cardinaux infinis ...................... 127

5.5.- Familles dénombrables quelconques $\ldots \ldots \ldots \ldots \ldots \ldots \ldots \ldots \ldots \ldots \ldots \ldots . \ldots 152$

Chapitre VI Un théorème d'échange ; circuits généralisés ; Applications à une conjecture de Rado .................. 154

6.1.- Le théorème d'échange pour un élément .................... 154

6.2.- Circuits généralisés ; La fonction $\mathrm{L}_{\mathbf{X}}$; Bases d'un circuit

6.3.- Un algorithme pour résoudre une conjecture de Rado ......... 166

Chapitre VII Changements de bases. Applications aux matrices ..... 175

7.1.- Lemmes et remarques préliminaires ...................... 175

7.2.- Changements de bases ................................ 178

7.3.- Propriétés des C.d.b. ................................. 180

7.4.- Applications aux matrices ............................. 184

7.5.- Notions plus fortes ................................ 185

Index terminologique $\quad \ldots \ldots \ldots \ldots \ldots \ldots \ldots \ldots \ldots \ldots \ldots \ldots \ldots \ldots \ldots \ldots \ldots \ldots \ldots \ldots \ldots \ldots$

Index des notations $\ldots \ldots \ldots \ldots \ldots \ldots \ldots \ldots \ldots \ldots \ldots \ldots \ldots \ldots \ldots \ldots \ldots \ldots \ldots \ldots \ldots \ldots$

Bibliographie $\ldots \ldots \ldots \ldots \ldots \ldots \ldots \ldots \ldots \ldots \ldots \ldots \ldots \ldots \ldots \ldots \ldots \ldots \ldots \ldots \ldots \ldots \ldots \ldots \ldots$ 
Introduction

Ce travail reprend en partie la rédaction de ma thèse. $\mathrm{Il}$ contient des résultats obtenus depuis, notamment dans les chapitres I, II, III et $\mathrm{V}$ qui ont été entièrement refondus.

L'objet primitif de ce travail a été l'étude du problème de géométrie algébrique suivant :

Soient pour $i=1, \ldots, n, M_{i}=\left(x_{i}^{j}\right)$ n points de l'espace affine $K^{p}$ sur le corps $K$ et $k$ un sous-corps de $K$. A quelles conditions peut-on choisir pour chaque point $M_{i}$ une de ses coordonnées $x_{i}^{j(i)}$, de sorte que les $n$ éléments de $\mathrm{K}$ ainsi choisis soient algébriquement libres sur $\mathrm{k}$ ?

La propriété :

$\forall J \subset\{1, \ldots, n\} \quad, \operatorname{dimal}_{k} k\left(M_{i} / i \in J\right) \geqslant \operatorname{Card} J$

s'est avérée nécessaire et suffisante pour qu'il en soit ainsi, d'où l'intérêt d'étudier cette propriété combinatoire associée à la dimension algébrique dans le cas d'autres dimensions.

Dans BOURBAKI $[\mathrm{C}, \mathrm{II}, 5], \S 5$, exercice $14, \mathrm{p} .108$ et dans Commutative Algebra de P. SAMUEL et 0. ZARISKI [L], chap. I, $\S 21$ et chap. II, $\S 12$ et 17 , est définie la structure de $\varphi$-espace pour étudier les propriétés communes des espaces vectoriels, des extensions de corps et des p-bases, propriétés associées aux notions de parties libres, de bases et de dimension.

La première partie de ce travail a donc pour but d'étudier les espèces de structures qui peuvent être définies en partant des notions de parties libres, bases, rang ou dimension, fonctions génératrices, fermetures algébriques et sous-espaces. Le langage fonctoriel étant désormais classique (voir J. BENABOU [P] et C. EHRESMANN [R]), et les espèces de structures, qui seront définies, étant 
espèces de structure au sens de BOURBAKI $[C, I, 4]$, le chapitre I a pour objet de dégager les "bonnes" structures grâce aux équivalences et aux déductions, qui sont des cas particuliers d'isomorphismes fonctoriels. Deux espèces de structure sont ainsi mises en évidence :

a) La structure de $\mathscr{L}$-espace : ensemble $\mathrm{E}$ muni de $\mathscr{L} \subset \dot{\beta}(\mathrm{E})$, de caractère fini (les éléments de $\mathscr{L}$ sont les parties libres).

b) La structure de $\varphi$-espace : $\mathscr{L}$-espace $E$ tel que, pour toute partie finie $X$ de $E$, les parties libres maximales incluses dans $X$ ont toutes le même nombre d'éléments.

Les planches I et II résument les systèmes d'axiomes équivalents et les équivalences canoniques de structures pour les $\mathscr{L}$-espaces et les $\varphi$-espaces. La planche III est un diagramme commutatif contenant un certain nombre de foncteurs fondamentaux définis dans le chapitre $I$.

Voici un bref historique concernant ces questions : Hassler WHITNEY dans [11] a étudié pour les ensembles finis des systèmes d'axiomes équivalents pour les notions de rang, base, partiesłibres, parties liées et bases complémentaires. En 1948, Richard RADO a etendu la notion de rang aux ensembles infinis dans [10] . Les structures définies sont essentiellement équivalentes à celle de $\varphi$-espace. Récemment P. SAMUEL et 0 . ZARISKI dans Commutative Algebra ont étudié une-structure équivalente définie à partir d'une fonction génératrice. Par ailleurs, l'étude des treillis géométriques ([E], IIIe Partie) et d'une manière générale les recherches sur les fondements de la géométrie (voir E.ARTIN [A] et R. BAER [0]) ont conduits à définir des espèces de structure plus ou moins équivalentes en partant des notions de sous-variétés ou de sous-espaces. Indiquons en outre les articles de HAUPT, NOBELING et PAUL [3] et de Richard RADO [9] où sont introduits des systèmes d'axiomes équivalents à ceux des $\varphi$-espaces, et le livre de Marshall HALL [F] chapitre 20, où sont étudiées en détail les géométries projectives planes. 
La notion de circuit (partie liée minimale), introduite par Hassler WHITNEY dans [11], simplifiera beaucoup de démonstrations. Cette terminologie est issue de la théorie des graphes (les graphes planaires sont canoniquement munis d'une structure de $\varphi$-espace, voir [12]).

Le chapitre II consiste en l'étude de ces deux espèces de structures grâce aux morphismes qui ont été définis au chapitre I. Les structures image réciproque et somme directe existent toujours, par contre, pour les $\varphi$-espaces les structures produit ou quotient n'existent pas en général. La réduction d'un $\varphi$-espace associée à l'image réciproque permet de préciser les liens entre les $\varphi$-espaces et les treillis géométriques. Quelques énoncés sur le problème de représentation terminent ce chapitre.

Le chapitre III est un chapitre Charnière entre cette première partie et les problèmes combinatoires. L'on rencontre le mot "disjonction" en théorie des ensembles (sous-ensembles disjoints), en théorie des corps (disjonction linéaire ou algébrique)... . Ces disjonctions sont de deux types : la disjonction linéaire est de nature initiale, i.e. est liée à la notion de produit, alors que la disjonction algébrique est de nature finale, i.e. est liée à la notion de réunion ou somme. D'où deux types de disjonction :

a) La disjonction simple, qui contient les disjonctions ensembliste et algébrique. Après avoir étudié cette disjonction dans les $\mathscr{L}$-espace, et notamment y avoir analysé la propriété de translation, l'on démontre des propriétés particulières à la disjonction dans les $\varphi$-espaces, propriétés qui sont déjả combinatoires

b) La disjonction double, qui contient la disjonction linéaire.

La deuxième partie a pour objet la résolution de problèmes combinatoires, finis ou infinis, sur les $\varphi$-espaces, voire les $\mathscr{L}$-espaces. Les chapitres IV et $\mathrm{V}$ sont consacrés au : 
Problème Etant donnée une famille de cardinaux $\left(a_{i}\right)_{i \in I}$, caractériser les familles $\left(x_{i}\right)_{i \in I}$ de parties d'un $\varphi$-espace $E$, pour qu'il existe une famille $\varphi$-disjointe $\left(\mathrm{Y}_{i}\right)$ incluse dans $\left(\mathrm{X}_{i}\right)$ (i.e. pour tout $\left.i, \mathrm{Y}_{i} \subset \mathrm{X}_{\mathbf{i}}\right)$ et telle que pour tout $i, \operatorname{dim} \varphi\left(Y_{i}\right)=a_{i} ;$ et construire une telle famille $\left(Y_{i}\right)$ quand elle existe.

$$
\begin{aligned}
& \text { Une condition nécessaire d'existence s'impose : } \\
& \forall \mathrm{J} \subset \mathrm{I}, \operatorname{dim} \varphi\left(\mathrm{x}_{\mathrm{J}}\right) \geq \mathrm{a}_{\mathrm{J}}
\end{aligned}
$$

où l'on écrit : $x_{J}=U x_{i}[i \in J]$ et $\quad a_{J}=\sum a_{i}[i \in J]$. L'on dit qu'une famille $\left(x_{i}\right)$ est de type $\left(a_{i}\right)$ quand la condition (1) est réalisée.

Au chapitre IV l'on démontre :

$\left.1^{\circ}\right)$ Si I est fini, toute famille $\left(x_{i}\right)$ de parties d'un $\varphi$-espace $E$, de type $\left(a_{i}\right)$ contient une famille $\psi$-disjointe de type $\left(a_{i}\right)$.

Quand les $a_{i}$ sont finis, deux démonstrations sont données, d'où deux méthodes (suppressibilité et empilement) pour construire une famille disjointe incluse et de type $\left(a_{i}\right)$

Ce résultat a pour cas particuliers le théorème de KONIG-HALL et un théorème de Richard RADO (voir $[10]$ ).

$2^{\circ}$ ) Le résultat $1^{\circ}$ ) est vrai dans un $\mathscr{L}$-espace $E$ si et seulement si $\mathrm{E}$ est un $\varphi$-espace.

Les familles infinies sont étudiées au chapitre $\mathrm{V}$. Voici les principaux théorèmes :

$\left.3^{\circ}\right)$ Toute famille $\left(x_{i}\right)$ de parties d'un $\varphi$-espace $E$, telle que pour tout $i$ $\operatorname{dim} \varphi\left(x_{i}\right)$ soit finie, contient une famille $\varphi$-disjointe de type $\left(a_{i}\right)$ si et seulement si :

$$
\forall J \in f(I) \quad, \quad \operatorname{dim} \varphi\left(x_{J}\right) \geqslant a_{J}
$$


où $\mathcal{F}_{(I)}$ est l'ensemble des parties finies de I.

Ceci généralise un théorème de Richard RADO (lemme 1 de [10])

$\left.4^{\circ}\right)$ Toute famille dénombrable $\left(x_{i}\right)$ telle que, pour tout $i \operatorname{dim} \varphi\left(x_{i}\right)$ soit infinie, contient une famille disjointe $\left(Y_{i}\right)$ telle que pour tout $i$,

$$
\operatorname{dim} \varphi\left(\mathrm{Y}_{\mathbf{i}}\right)=\operatorname{dim} \varphi\left(\mathrm{X}_{\mathbf{i}}\right)
$$

(Pour les familles telles que Card $I \geq \chi_{1}$, cet énoncé est faux en général).

$\left.5^{\circ}\right)$ Si l'on admet 'seulement l'axiome du choix, l'on a : Soit pour une famille $\left(a_{i}\right)_{i \in I}$ de cardinaux infinis, $L$ l'ensemble des $a_{i}$ et les propriétés :

(a) Pour tout cardinal $l \in \mathrm{L}$ tel que $l=U_{0}$ ou $l$ soit singulier, l'on a : Card $\left\{i \mid a_{i}=\ell\right\} \leq \hat{\ell}$

(b) Pour tout cardinal $l \in \mathrm{L}$ tel que $l \neq U_{0}$ et $l$ soit régulier, l'on a : Card $\left\{\mathbf{i} \mid \mathbf{a}_{\mathbf{i}}=\ell\right\}<\ell$

(c) Toute partie $X$ de $L$ telle que :

$$
\forall \mathrm{Y} \subset \mathrm{X} \quad, \quad \sup \mathrm{Y} \in \mathrm{X} \cup\{\operatorname{Sup} \mathrm{X}\}
$$

vérifie nrd $(X)<\omega_{1}$

(d) L'ensemble des cardinaux $\ell$ de $L$ tels que :

$$
U_{0}<\bar{l}<l \text { et } \sup \mathrm{L} \cap(] \leftarrow, \ell[)=l
$$

où $\bar{l}$ est le cardinal de cofinalité de $l$ et $]_{-}, l[l$ 'ensemble des cardinaux $<\ell$, a un cardinal strictement inférieur à $U_{2}$.

Toute famille $\left(X_{i}\right)$ de parties d'un $\varphi$-espace $E$ telle que dim $\varphi\left(X_{i}\right)$ soit infinie pour tout $i$ et telle que $\left(\operatorname{dim} \varphi\left(X_{i}\right)\right)_{i \in I}$ vérifie les propriétés (a), (b), (c) et (d), contient une famille $\varphi$-disjointe $\left(Y_{i}\right)$ telle que : pour tout $i \in I, \quad \operatorname{dim} \varphi\left(\mathrm{Y}_{\mathbf{i}}\right)=\operatorname{dim} \varphi\left(\mathrm{X}_{\mathbf{i}}\right)$ 
(Les conditions (a), (b), (c) et (d) sont restrictives, et seuls des cas marginaux signalés au passage, sont restés ouverts).

$\left.6^{\circ}\right)$ Si l'on admet l'axiome du choix et $l^{\prime h y p o t h e ̀ s e ~ d u ~ c o n t i n u ~ g e ́ n e ́ r a l i s e ́, ~}$ toute famille $\left(x_{i}\right)_{i \in I}$ de parties d'un $\varphi$-espace $E$ telle que, pour tout $i \in I$, $\operatorname{dim} \varphi\left(\mathbf{X}_{\mathbf{i}}\right)$ soit infinie, et telle que la famille $\left(\operatorname{dim} \varphi\left(\mathbf{X}_{\mathbf{i}}\right)\right)_{\mathbf{i} \in I}$ vérifie les propriétés (c), (d) et :

$$
\text { (a') } \forall l \in \mathrm{L}, \operatorname{Card}\left\{\mathbf{i} \mid \mathbf{a}_{\mathbf{i}}=\ell\right\} \leq l
$$

contient une famille $\varphi$-disjointe $\left(\mathrm{Y}_{i}\right)$ telle que :

pour tout $\mathbf{i} \in \mathrm{I}, \operatorname{dim} \varphi\left(\mathbf{X}_{\mathbf{i}}\right)=\operatorname{dim} \varphi\left(\mathbf{Y}_{\mathbf{i}}\right)$.

Ce chapitre se termine par une étude complète des familles dénombra-

bles.

Au chapitre suivant, après avoir examiné la manière dont peut s'échanger un élément d'une base donnée avec un autre élément pour obtenir une nouvelle base, l'on étudie les circuits généralisés, i.e. les ensembles $\mathscr{C}$ de circuits tels qu'il existe une partie libre $L$ pour laquelle les circuits de $\mathscr{C}$ ont au plus un élément en dehors de $L$. Quand $L$ est fixée, la correspondance galoisienne $: \mathscr{C} \longrightarrow \cup \mathscr{C}_{-} \mathrm{L}$ est un isomorphisme de l'ensemble des circuits généralisés définis par $L$ sur $\beta(\varphi(L)-L)$ ordonnés par l'inclusion. D'où la fonction $\mathrm{L}_{\mathrm{X}}=\cup \mathrm{L}_{\mathbf{X}}[\mathrm{x} \in \mathrm{X}]$. L'on a ainsi une généralisation des "Systèmes fondamentaux de circuits" de Hassler WHITNEY. Une propriété caractéristique combinatoire des circuits généralisés est exposée par le théorème 4. Des applications à des constructions de $\varphi$-espaces permettent d'améliorer un résultat de $\mathrm{H}$. WHITNEY ([11], Appendice) concernant les $\varphi$-espaces $\mathbb{F}_{2}$-représentables. Avec ces techniques, l'on démontre alors :

Soit $E$ un $\varphi$-espace et $k$ un entier $\geqslant 1$. Pour qu'une partie $S$ de $E$ admette une partition formée de $k$ parties libres, il faut et il suffit que $S$ vérifie l'une des propriétés équivalentes :

$\left(P_{k}\right) \quad$ Card $S \geq k$ et pour tout $x \in F(S), \operatorname{Card} X \leq k \cdot \operatorname{dim} \varphi(x)$

$\left(P_{k}^{\prime}\right)$ Card $S \geqslant k$ et pour tout sous-espace A de dimension finie, Card $(A \cap S) \leqslant k$. $\operatorname{dim} \varphi(A \cap S)$

Ayant démontré ce théorème sur les espaces vectoriels, $M$. RADO se demandait si une condition nécessaire et suffisante de représentabilité d'un $\psi$-espace comme partie d'un espace vectoriel n'était pas la validité de ce théorème. 
Le dernier chapitre est une théorie constructive des changements de base. Pour les définir, il a été nécessaire d'établir le leme combinatoire de 7.1. L'importance des changements de base faibles est qu'on peut en démontrer l'existence pour tout ordinal dénombrable....

Plus précisément :

Soient $X$ et $L$ deux bases d'un $\varphi$-espace $E$, $\leq$ un bon ordre sur $X$. Si Ord $(x, \leq)<\omega_{1}$, alors il existe au moins une injection $u$ de $x$ dans $L$ telle que : $\mathrm{L}-\mathrm{u}(\mathrm{X})+\mathrm{X}$ soit une base de $\mathrm{E}$ et $\forall \mathbf{x} \in \mathrm{X},\left(\mathrm{L}-\mathrm{u}\left(J_{\leftarrow, \mathbf{x}}[)\right) \cup(\rfloor_{\leftarrow,} \mathbf{x}\right)$ soit une base de $E$.

Quelques considérations sur des notions plus fortes et des exemples sur les matrices montrent la complexité de ces questions.

Je tiens à remercier vivement Monsieur le Professeur SAMUEL, mon directeur de thèse, pour les conseils avisés et les encouragements qu'il m'a constamment prodigués, et pour la confiance qu'il m'a toujours témoignée. Ce travail lui doit beaucoup et je le prie d'accepter ma sincère gratitude, ainsi que Monsieur le Professeur BENZECRI, qui m'a engagé dans la recherche.

Je prie Monsieur le Recteur MARTIN et Monsieur le Doyen BOCLE, mes premiers maîtres, qui m'ont conseillés depuis mes débuts, d'agréer l'hommage de mon profond respect et de ma sincère reconnaissance.

J'exprime ma sincère gratitude à Messieurs les Professeurs GUERINDON et GLAESER, et à Monsieur BENABOU pour leur aide et pour l'intérêt qu'ils ont porté à mon travail.

Enfin je ne saurais non plus oublier l'aide et les encouragements de Messieurs les Professeurs ANTOINE et METIVIER, ainsi que de tous ceux qui m'ont entouré au cours de mes études. Que tous veuillent bien croire à ma reconnaissance. 


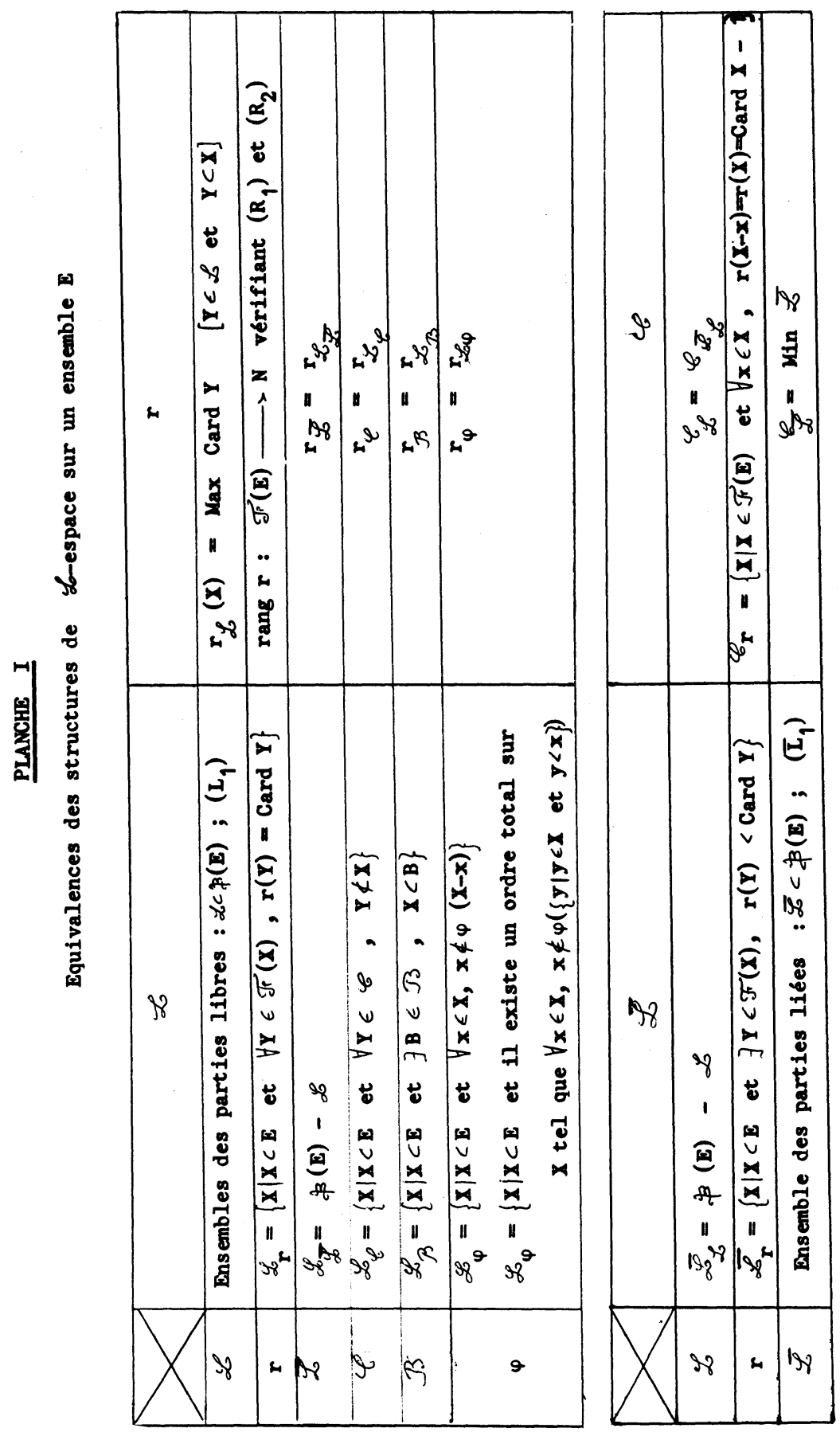



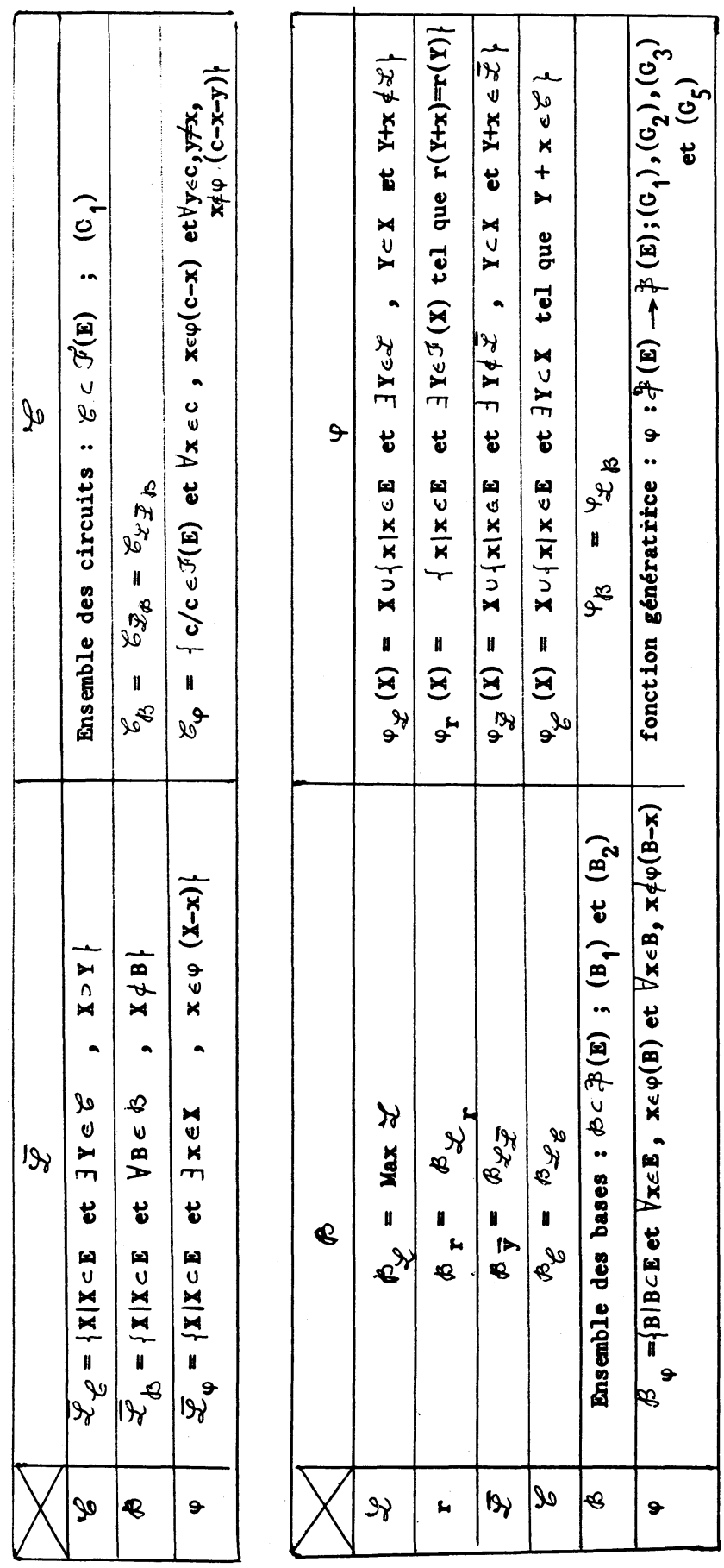

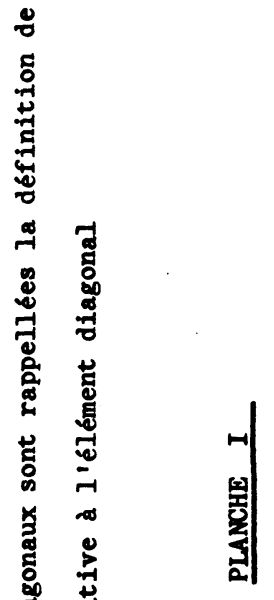



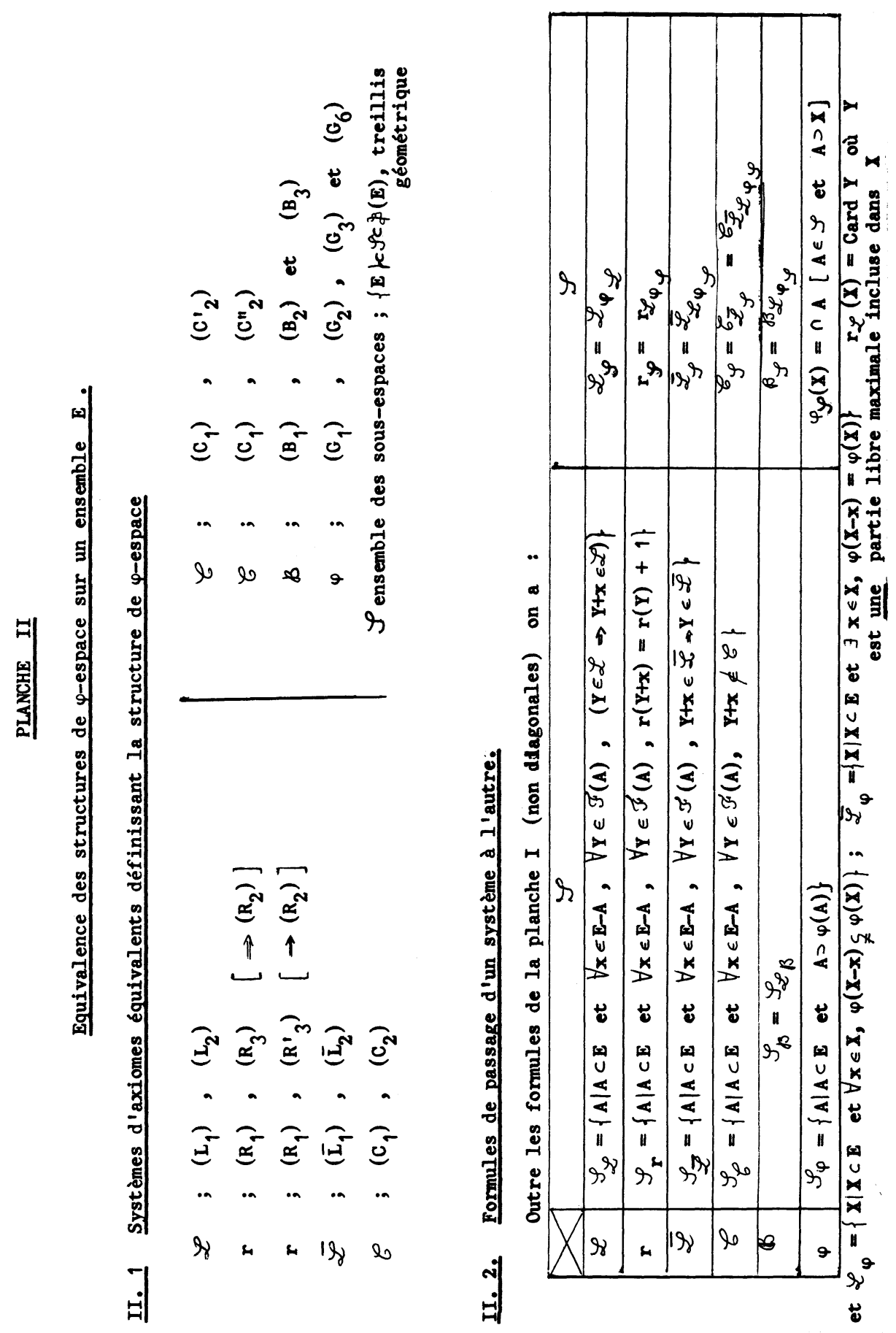


\section{Diagramme récapitulatif des principaux facteurs définis} au chapitre $\mathbf{I}$.

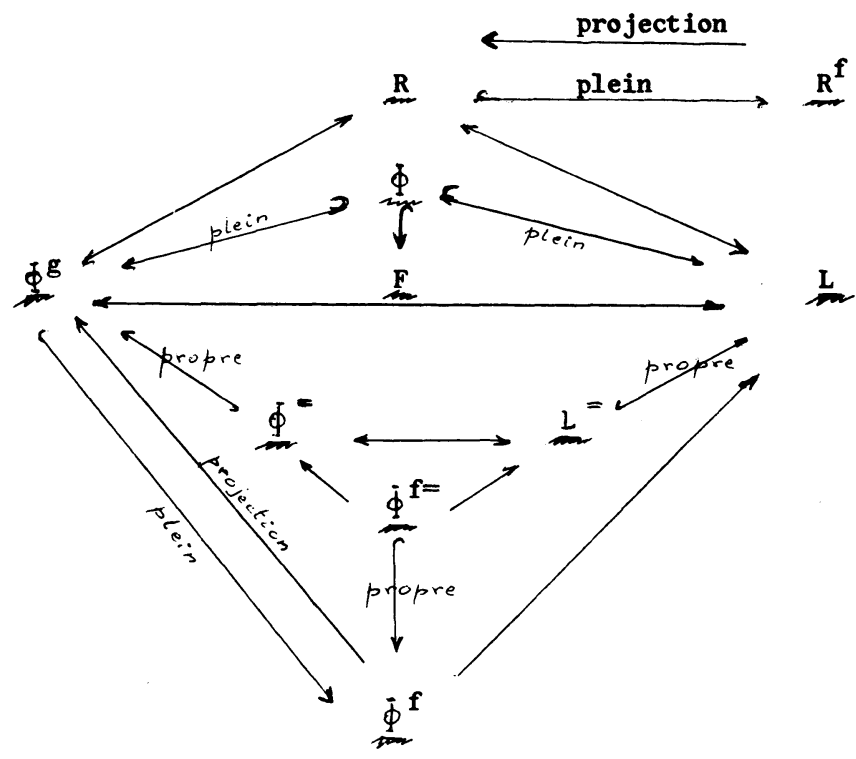

Ce diagramme est commutatif, les flèches représentant des fonct eurs ensemblistes.

$\longleftrightarrow$ sont des isomorphismes

$\longleftrightarrow$ sont des foncteurs d'inclusion, ou des foncteurs injectifs 
Chap. I Axiomatique sur les parties libres

et les parties ou familles génératrices

\subsection{Introduction}

Les notions de parties libres, rang, partie liée, circuit, fonctions génératrices, fermetures et treillis sont du langage courant de nombre de théories dans la Mathématique. L'objet de cette première section est de définir les structures naturelles obtenues en généralisant ces notions dont les cas particuliers sont bien connus et de comparer les diverses généralisations obtenues. Il se dégage de cette étude deux structures importantes :

10) $\mathscr{L}$-espace : couple $(E, \mathscr{L})$ formé d'un ensemble $E$ et d'une partie $\mathscr{L}$ de $\beta(E)$, de caractère fini.

$\left.2^{\circ}\right) \varphi$-espace : couple $(E, \varphi)$ formé d'un ensemble $E$ et d'une fermeture $\varphi$, de caractère fini et vérifiant un axiome d'échange qui' sera précisé.

Certaines des équivalences de structure qui sont présentées ont été étudiées par $H$. Whitney $\left[{ }^{11}\right]$ pour les ensembles finis, R. Rado [10] pour les rangs, B. Jönsson [4] pour les fermetures et les treillis, ... •

Voici quelques précisions sur le langage utilisé pour les équivalences de structures et de catégories

Pour éviter toute difficulté de logique, tous les ensembles considérés sont supposés appartenir à un mème ensemble universel dans lequel on peut faire les opérations ensemblistes usuelles (voir C. Ehresmann [ $R]$, introduction, pp. VIII et IX), et Ens est de la catégorie des applications entre ces ensembles.

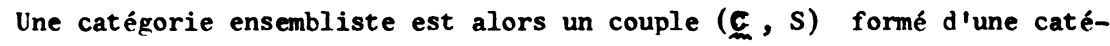
gorie $\mathbb{C}$ et d'un foncteur fidèle $S$ de $\underset{\mathbb{C} \text { dans }}{\text { Ens }}$ appellé foncteur d'oubli. Un foncteur ensembliste de $(\mathscr{L}, S)$ dans $\left(\mathfrak{w}^{\prime}, S^{\prime}\right)$ est alors un foncteur $F$ de $\underset{c}{c}$

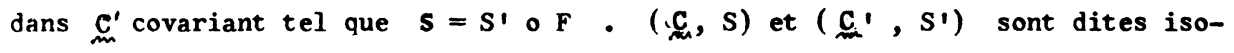
morphes si et seulement si il existe un isomorphisme ensembliste de sur

Si les objets de $\underset{m}{c}$ (resp. $\left.\mathcal{C}^{\prime}\right)$ sont des ensembles structurés par une

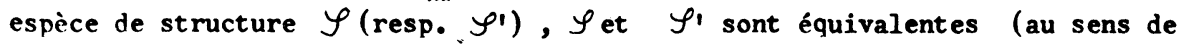
Bourbaki $\left.[C, I, 4], \oint_{1, n^{\circ}} 7\right)$ si et seulement si (I c 
sont isomorphes (I 대 sous-catégorie des isomorphismes de $\underset{m}{\mathrm{e}}$ ). Voici en outre quelques propriétés :

1) Un foncteur ensembliste est déterminé par les transformés des objets (on en déduit aisément alors les transformés des morphismes).

2) Si $F$ et $G$ sont ensemblistes et si $F \circ G$ existe, $F \circ G$ l'est aussi.

3) Si $F$ est ensembliste et inversible, alors $F^{-1}$ est ensembliste.

Ces notions s'étendent aisément aux foncteurs ensemblistes contravariants.

Pour tout ensemble E, l'on écrit C (E) l'ensemble ordonné des objets au-dessus de $E$ : $A \geqslant B$ si et seulement si l'application identique de $E$ est un morphisme de A dans $B$ ( $s i{ }_{E}$ isomorphisme de $A$ sur $B$ entraine $A=B$ sinon préordre seulement).

\section{2. - Parties libres, rangs, parties liées, bases et circuits}

\subsection{1.- $\mathscr{L}$-espaces}

Définition 1.- L'on appelle $\mathscr{L}$-espace tout couple $(E, \mathscr{L})$ formé d'un ensemble E et d'une partie $\mathcal{L}$ de $\xi(E)$, de caractère fini, i.e. vérifiant l'axiome * :

$$
\left(\mathrm{L}_{1}\right) \quad(\forall \mathrm{X} \in \mathbb{B}(\mathrm{E})) \quad\left((\mathrm{X} \in \mathcal{Z}) \Longleftrightarrow\left(\forall \mathrm{Y} \in \mathcal{F}^{(\mathrm{X})}, \mathrm{Y} \in \mathcal{Z}\right)\right)
$$

Les $\mathrm{x} \in \mathcal{Z}$ sont alors appellées des parties libres (ou $\mathscr{L}$-libres)

Si $X$ est libre, toute partie de $\mathrm{X}$ est libre.

Exemples :

$1^{\circ)}$ Tout A-module à gauche $M$. L'on peut définir entre autres les ensembles de parties libres suivant :

$$
\text { 1 a) } \mathscr{L}_{0}=\{x \mid x \in M \quad \text { et } \quad \forall x \in X, x \notin \overline{x-\{x}\}
$$

où $\overline{\mathrm{X}-\{\mathrm{x}\}}$ est le sous-A-module engendré par $\mathrm{X}-\{\mathrm{x}\}$. Ce sont les parties líbreses_classsiques.

* $f(x)$ est l'ensemble des parties finies de $x$

$f_{p}(x)$ est l'ensemble des parties de cardinal $p$, de $x$ 
1 b) $\mathscr{L}_{1}=\left\{x \mid x \in M \quad\right.$ et $\left.\quad\left(\left(\sum \lambda_{x} x[x \in X]=0\right) \rightarrow\left(\forall x \in X, \lambda_{x} x=0\right)\right)\right\}$ où $\sum$ est une combinaison A-linéaire.

Quand $M$ est unitaire, $\mathscr{L}_{1} \supset \mathscr{L}_{0} ;$ mais $\mathscr{L}_{1}=\mathscr{L}_{0}$ si et seulement si $\mathrm{A} / \mathrm{I}$ est un corps où $\mathrm{I}$ est l'annulateur de $M$. (voir $[\mathrm{C}, \mathrm{II}, 2], \mathrm{n}^{\circ} 12, \mathrm{p} .45$ ).

$2^{\circ}$ ) Soit B sous-anneau de $\mathrm{A}, \mathscr{L}_{0}$ (resp. $\mathscr{L}_{1}$ ) l'ensemble des parties X de A telles que **:

$$
\begin{aligned}
& \forall \mathrm{y}=\left\{\mathrm{y}_{1}, \ldots, \mathrm{y}_{\mathrm{n}}\right\} \in \mathcal{F}(\mathrm{x}), \forall \mathrm{f} \in \mathrm{B} \quad\left[\mathrm{x}_{1}, \ldots, \mathrm{x}_{\mathrm{n}}\right] \\
& \mathbf{f}\left(\mathrm{y}_{1}, \ldots, \mathrm{y}_{\mathbf{n}}\right)=0 \quad \text { entraine } \mathrm{f}=0
\end{aligned}
$$

(resp. $\forall x \in X, \quad \forall y=\left\{y_{1}, \ldots, y_{n}\right\} \in \zeta^{\prime}(X-\{x\})$, il $n$ 'existe pas de polynômes $f \in B \quad\left[x_{0}, x_{1}, \ldots, x_{n}\right]$ distingué en $x_{0}$ tel que $: f\left(x, y_{1}, \ldots, y_{n}\right)=0$ (l'on suppose ici que $B$ a une unité e qui est le coefficient du terme de plus haut degré en $x_{0}$ ) ).

Les parties dans $\mathscr{L}_{0}$ sont dites algèbriquement libres sur B, celles dans $\mathscr{L}_{1}$ intégralement libres sur B.

$3^{\circ}$ ) De tout schema simplicial S (ou complexe simplicial) (voir[M], chap. I, $3.2 .$, p. 37) on déduit canoniquement un $\mathscr{L}$-espace : soit $\mathcal{Y}$ l'ensemble des simplexes de $S, \mathscr{L}$ est $l$ 'ensemble des parties $X$ de $S$ telles que :

$\forall \mathrm{Y} \in \mathscr{F}(\mathrm{X}),(\mathrm{y})_{\mathrm{y} \in \mathrm{Y}}$ soit un simplexe.

Une famille $\left(x_{i}\right)_{i \in I}$ d'éléments d'un $\mathscr{L}$-espace est dite libre si et seulement si les $x_{i}$ sont distincts deux à deux et $\left\{x_{i} \mid i \in I\right\}$ est une partie $\mathscr{L}$-libre.

L'on désigne par $\underset{m}{\mathrm{~m}}$ la catégorie dont les objets sont les $\mathscr{Z}$-espaces, et dont les morphismes sont les applications $f$ de $(E, \mathscr{L})$ dans $\left(E^{\prime}, \mathscr{L}^{\prime}\right)$ telles que :

$\left(h_{q}\right)$ toute famille $\left(x_{i}\right)$ d'éléments de F. telle que $\left(f\left(x_{i}\right)\right)$ soit LL-libre, est $\mathscr{L}$-libre

(on généralise ainsi la notion d'application linéaire).

* * $B\left[x_{1}, \ldots, x_{n}\right]$ est l'anneau des polỵnômes ̀ $n$ indéterminées $x_{1}, \ldots, x_{n}$ sur $B$ 


\subsection{2.- rang sur $(E, \mathscr{L})$. Rangs faibles.} nombre entier :

L'application $r_{\mathscr{L}}\left(\right.$ ou $\left.r_{\mathscr{L}}\right)$ qui à toute partie $X$ finie de $E$ associe le

$$
\mathbf{r}_{\mathscr{L}}(\mathrm{X})=\mathbf{r}(\mathrm{X})=\operatorname{Max}(|\mathrm{Y}|) \quad[\mathrm{Y} \in \mathcal{L} \quad \text { et } \quad \mathrm{Y} \subset \mathrm{X}]
$$

vérifie les axiomes *

$$
\begin{aligned}
& \left(R_{1}\right) \quad r(\varnothing)=0 \text { et } \forall x \in \mathscr{F}(E), \forall x \in E, r(x+x)=r(x)+h \text { où } h=0 \\
& \text { ou } h=1 \\
& \left(R_{2}\right) \quad \forall x \in \mathscr{F}(E), \quad \exists y \in \mathscr{F}(x), \quad r(X)=r(Y)=|y|
\end{aligned}
$$

Définition 2.- On appelle rang (resp. rang faible) sur un ensemble E toute application $r$ de $\mathcal{F}^{\prime}(E)$ dans $l$ 'ensemble $N$ des entiers naturels vérifiant les axiomes $\left(R_{q}\right)$ et $\left(R_{2}\right) \quad\left(\right.$ resp. $\left.\left(R_{q}\right)\right)$ ci-dessus.

Il résulte de l'axiome $\left(R_{1}\right)$ que $: \forall x \in \mathcal{F}(E), 0 \leq r(x) \leq|x|$

L'on désigne par $\underset{m}{\mathrm{R}}$ (resp. R) $^{\mathrm{R}}$ la catégorie dont les objets sont les ensembles $(E, r)$ munis d'un rang faible (resp. rang) $r$, les morphismes de $(E, r)$ dans $\left(E^{\prime}, r^{\prime}\right)$ étant les applications $f$ de $E$ dans $E^{\prime}$ qui diminuent le rang, i.e. telles que :

$$
\forall x \in F^{\prime}(E), \quad r^{\prime}(f(x)) \leqslant r(x)
$$

Théorème 1.- $(E, r) \longrightarrow\left(E, \mathscr{L}_{r}\right)$

$$
\text { où } \quad \mathscr{L}_{\mathbf{r}}=\{\mathrm{X} \mid \mathrm{X} \subset \mathrm{E} \quad \text { et } \quad \forall \mathrm{Y} \in \mathscr{F}(\mathrm{X}), \mathrm{r}(\mathrm{Y})=|\mathrm{Y}|\}
$$

définit un foncteur ensembliste de $\underset{m}{\underset{f}{f}}$ sur $\underset{m}{L}$ dont la restriction à $\underset{\sim}{\mathrm{R}}$ est un isomorphisme de $\underset{m}{R}$ sur $\mathrm{Lm}_{\mathrm{m}} \mathrm{L}^{\prime}$ isomorphisme inverse est défini par la relation (1).

Démonstration : On vérifie que :

$\left.1^{\circ}\right)$ E étant un ensemble donné, $r \rightarrow \mathscr{L}_{r}$ défini par (2) est une application de $\underset{m}{\mathbf{f}}(E)$ dans $\underset{m}{\mathbf{L}}(E)$ qui $n$ 'est pas croissante en général, mais dont la restriction à $\underset{m}{R}(E)$ est un isomorphisme d'ensembles ordonnés de $\underset{m}{R}(E)$ sur $\underset{m}{L}(E)$, ce dernier point étant d'ailleurs une conséquence de :

L'on écrit $|Y|$ au lieu de Card $Y$ quand aucune confusion n'est à craindre avec une valuation. 


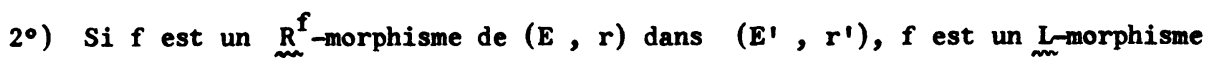
de $\left(E, \mathscr{L}_{r}\right)$ dans $\left(E^{\prime}, \mathscr{L}_{r^{\prime}}\right)$ (réciproque fausse en général), mais $f$ est un R-morphisme si et seulement si $f$ est un $\underset{m}{\mathrm{~L}}$-morphisme

D'où la correspondance galoisienne :

Corollaire 1.- Soit E un ensemble $r \longrightarrow \mathscr{L}_{r}$ et $\mathscr{L} \longrightarrow r \mathscr{L}$ sont des isomorphismes inverses l'un de l'autre de l'ensemble $\underset{m}{\mathrm{R}(E)}$ ordonné par $\mathbf{r}_{1} \leqslant \mathbf{r}_{2}$ si et seulement si $\forall x \in F^{\prime}(E), r_{1}(X) \leq r_{2}(X)$, sur $L(E)$ ordonné par : $\mathscr{L}_{1} \subset \mathscr{L}_{2}$ (inclusion dans $₹(\xi(E))$ ).

Corollaire 2.- Les espèces de structure définies respectivement par : $\left(E, \mathcal{L},\left(L_{1}\right)\right)$ et $\left(E, r,\left(R_{1}\right)\right.$ et $\left.\left(R_{2}\right)\right)$ sont équivalentes.

L'énoncé du théorème 1 s'exprime aussi en disant que la diagramme (I) est commutatif, où $I$ et $I^{-1}$ sont les isomorphismes entre $\underset{m}{R}$ et $\underset{m}{L}, \quad F$ le foncteur ensembliste de $\underset{m}{f}$ sur $I, P=I$ o $F$ le foncteur projection de $\mathrm{R}^{f}$ sur $\underset{m}{R}(P \circ P=P) e t \hookrightarrow$ le foncteur d'inclusion de $R$ dans $\mathbf{R}_{\mathrm{m}}^{\mathbf{f}}$

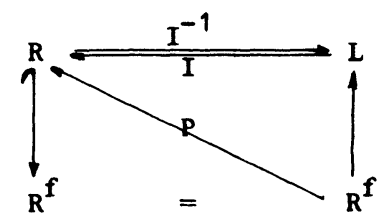

(I)

En particulier notons le :

Corollaire 3.- Soit $E$ un ensemble. $\mathbf{r} \longrightarrow \hat{r}=\mathbf{r}_{\mathcal{L}_{\mathbf{r}}}$ est une projection croissante de $\underset{m}{R^{f}}(E)$ sur $\underset{n}{R}(E)$. $\hat{r}$ est appellé le rang associé au rang faible $r$.

Prolongement du rang aux parties infinies :

La formule (1) de 1.2.2. permet de prolonger $r$ en une application de $\$$ (E) dans $I^{\prime}$ 'ensemb]e des cardinaux $\leqslant|E|$ :

$$
r(X)=\operatorname{Max}|Y| \quad[Y \subset X \quad \text { et } \quad Y \in \mathscr{Z}]
$$

c'est le prolongement canonique de $r$ à $\not(E)$. Les propriétés $\left(R_{1}\right)$ et $\left(R_{2}\right)$ restert vraies en remplaçant $\mathcal{F}(E)$ et $\mathcal{F}(X)$ par $\beta(E)$ et $\xi(X)$ respectivement. Par contre la propriété : "une partie finie $X$ est libre si et seulement si $r(X)=|X| "$, n'est plus vraie pour les parties infinies.

\subsection{3.- Parties liées, circuits et bases d'un $\mathscr{L}$-espace}

Définition 3.- Soit $\mathrm{E}$ un $\mathscr{L}$-espace. On appelle partie (resp. famille) $\mathscr{L}$-liée (ou plus simplement liée) toute partie (resp. famille) non $\mathscr{L}$-libre. L'on appelle circuit toute partie $\mathscr{L}$-liée minimale et base toute partie $\mathscr{L}$-libremaximale. 


$$
\begin{gathered}
\overline{\mathscr{L}}=\bar{\beta}(\mathrm{E})-\mathscr{L} \text { est l'ensemble des parties liées et vérifie : } \\
\left(\overline{\mathrm{L}}_{q}\right) \overline{\mathscr{L}} \subset \bar{\beta}(\mathrm{E})-\{\varnothing\} \text { et }(\forall \mathrm{x} \in \bar{\not}(\mathrm{E})) \quad((\mathrm{x} \in \overline{\mathcal{L}}) \Longleftrightarrow(\mathcal{F}(\mathrm{x}) \cap \overline{\mathcal{L}} \neq \varnothing))
\end{gathered}
$$

Alors toute partie liée contient une partie liée finie, donc une partie liée minimale. L'ensemble $\mathscr{C}$ des circuits vérifie :

(c, $) \quad \mathscr{C} \subset \mathscr{F}(E)-\{\varnothing\}$ et deux éléments distincts de $\mathscr{C}$ ne sont pas comparables (pour l'inclusion).

$\mathscr{L}$ étant de caractère fini est inductif pour l'inclusion (voir par exemple Bourbaki $[\mathrm{C}, \mathrm{I}, 3],\{4,5, \mathrm{p} .70)$, donc admet des éléments maximaux et toute partie libre est incluse dans une base. L'ensemble $B$ des bases vérifie l'axiome : $\left(B_{1}\right) \quad \beta \neq \varnothing, \quad B \subset \bar{\beta}(E)$ et deux éléments distincts ne sont pas comparables.

Cet axiome ne suffit pas pour reconstruire une $\mathscr{L}$ de caractère fini. En effet $B$ est tel que, pour tout $\mathrm{X} \in \mathrm{E}$,

$$
\beta_{x}=\mathscr{L} \cap \beta(x)
$$

est inductif pour l'inclusion, et il est aisé de construire des $B$ vérifiant $\left(B_{1}\right)$ et ne possèdant pas cette dernière propriété. D'où la nécessité du second axiome :

$\left(B_{2}\right) \quad\left(B\right.$ est de caractère fini) : $\forall x \in \beta(E), B_{X}=\{B \cap x \mid B \in B\}$ est inductif pour l'inclusion.

Il est aisé de vérifier que, sur un ensemble $E$ quelconque, les structures suivantes sont équivalentes :

1) L vérifiant $\left(L_{q}\right)$

2) $r$ rang sur $E$

3) $\bar{\Psi}$ vérifiant $\left(\overline{\mathrm{L}}_{q}\right)$

4) $€$ vérifiant $\left(C_{1}\right)$

$5)^{*} B$ vérifiant $\left(B_{1}\right)$ et $\left(B_{2}\right)$

* L'équivalence utilise le résultat suivant :

soit $\mathscr{L} \subset \beta(E)$ telle que : $\forall \mathrm{X} \in \mathscr{L}, \forall \mathrm{y} \in \not{\beta}(\mathrm{x}), \quad \mathrm{y} \in \mathscr{L}$

Alors $\mathscr{L}$ est inductive pour l'inclusion si et seulement si $\mathscr{L}$ est de caractère

fini. (Démonstration aisée par récurrence transfinie pour la condition nécessaire) 
ces équivalences : $\mathscr{L}_{\mathbf{r}}, \mathscr{L}_{\mathcal{Z}}, \mathscr{L}_{\mathscr{L}}, \mathscr{L}_{\beta}, \mathbf{r}_{\mathscr{L}}, \mathbf{r}_{\bar{L}}, \ldots \quad$ étant explicitées sur la planche $I$.

Résumons les résultats les plus intéressants :

proposition 1.- Dans un $\mathscr{L}$-espace $E$, une partie est liée si et seulement si elle contient un circuit ; une partie est libre si et seulement si elle est incluse dans une base; une partie finie $X$ est un circuit si et seulement si :

$$
\forall x \in X \quad, \quad r(x-x)=r(x)=|x|-1
$$

Les $\underset{m}{L}$-morphismes sont en outres caractérisés par l'une des conditions équivalentes :

$$
\begin{array}{llllll}
\left(h_{3}\right) & \text { si }\left(x_{i}\right) \text { est } \mathscr{L} \text {-liée, }\left(f\left(x_{i}\right)\right) \text { est } \mathscr{L}^{\prime} \text {-liée } \\
\left(h_{4}\right) \text { si }\left(x_{i}\right) \text { est un } \mathscr{L} \text {-circuit, }\left(f\left(x_{i}\right)\right) \text { est } \mathscr{L}^{\prime} \text {-liée }
\end{array}
$$

\subsection{4.- Rangs réguliers. Axiomes de Régularités.}

L'on constate pour l'instant une analogie assez complète entre les notions définies sur un $\mathscr{L}$-espace et celles qui sont couramment utilisées dans un espace vectoriel par exemple. Cependant la propriété fondamentale du rang dans un espace vectoriel n'est pas vérifiée en général. Plus précisément :

Définition 4.- Un rang faible $r$ sur un ensemble $E$ est dit régulier s'il vérifie la propriété suivante :

$$
\forall x \in \mathcal{F}(\mathrm{E}) \text {, toute partie libre incluse dans } \mathrm{x} \text { et maximale a pour }
$$
cardinal $\mathbf{r}(\mathrm{X})$.

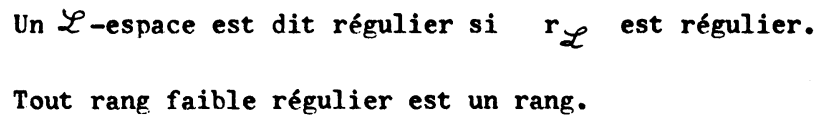

Si $r$ est un rang régulier, pour calculer $r(X)$, il suffit de construire une partie libre maximale incluse dans $X$. Le rang de $X$ est alors le cardinal de cette partie libre maximale.

Théorème 2.- Soit $r$ un rang faible sur un ensemble $E$. Les propriétés suivantes sont équivalentes :

$(R R) \quad r$ est un rang régulier

(R 3) $\forall X \in \mathcal{X}(E), \forall Y \in f(E)$ tels que $r(X \cup Y)=r(X)+1, \exists$ yeY tel que $r(X+y)=r(X)+1$

( $\left.R^{\prime}, 3\right) \forall x \in \mathcal{F}(E), \forall x \in E$ et $\forall y \in E$ tels que $r(x+x)=r(x+y)=r(X)$, on a $r(x+x+y)=r(x)$. 


\section{Démonstration}

10) Si $r$ est un rang faible vérifiant ( $\left.R^{\prime} 3\right)$ :

$\forall x \in F(E), \forall z \in F(x)$ tels que $\forall x \in X-z, \quad r(z+x)=r(z)$, on $a$ $r(X)=r(z) \quad$ (raisonner par récurrence sur $|X-z|)$. En particulier pour toute partie libre maximale $z$ incluse dans $x, r(x)=r(z)=|z|$. Donc $r$ est un rang régulier, et l'on démontre de même l'axiome $\left(R_{3}\right)$.

$\left.2^{\circ}\right)$ Si $r$ est un rang faible vérifiant $\left(R_{3}\right)$ :

Soit $X \in \mathcal{F}(E)$ et $Y$ une partie libre maximale incluse dans $X$. Alors si $r(X)>|Y|$, il existe $T \subset X-Y$ tel que $r(Y+T)=r(Y)+1=|Y|+1$, donc il existerait $y \in T$ tel que $r(Y+y)=|Y|+1: Y+y$ serait libre, ce qui contredit le caractère maximal de $Y$. Donc $r(X)=|Y|=r(Y)$ et $r$ est un rang régulier.

$\left.3^{\circ}\right)$ Si $r$ est un rang régulier :

$\forall \mathrm{x} \in \mathbb{F}(\mathrm{E}), \forall \mathrm{x} \in \mathrm{E}, \forall \mathrm{y} \in \mathrm{E}$ tels que $\mathrm{r}(\mathrm{X}+\mathrm{x})=\mathrm{r}(\mathrm{X}+\mathrm{y})=\mathrm{r}(\mathrm{X})$, on $\mathrm{a}:$ soit $E$ une partie libre maximale incluse dans $X: r(X)=|z|=r(z)$. Alors $r(z+x)=r(z+y)=r(x)=|z|$, donc $z+x$ et $z+y$ sont liés, et $z$ est une partie libre maximale incluoe dans $x+x+y$

D'où $r(x+x+y)=|z|=r(x)$ : $r$ vérifie $\left(R_{3}\right)$

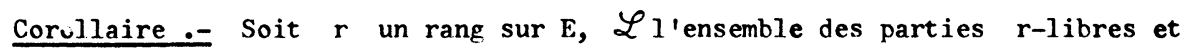
l'ensemble des parties r-liées. Les propriétés suivantes sont équivalentes

$(R R) \quad r$ est un rang régulier

$\left(\mathrm{L}_{2}\right) \quad \forall \mathrm{x} \in \mathscr{L}, \forall \mathrm{y} \in \mathscr{L}$ tels que $|\mathrm{X}|=|\mathrm{y}|+1$ soit fini, $\exists \mathrm{x} \in \mathrm{X}-\mathrm{Y}$ tel que $\mathrm{y}+\mathrm{x} \in \mathscr{L}$

$\left(\overline{\mathrm{I}}_{2}\right) \quad \forall \mathrm{X} \in \mathcal{F}(\mathrm{E}), \forall \mathrm{Y} \in \mathcal{F}(\mathrm{E})$ tels que $|\mathrm{X}|=|\mathrm{Y}|+1$ et $\mathrm{Y} \notin \overline{\mathscr{L}}$, on a : $(\forall \mathbf{X} \in \mathrm{X}, \mathrm{Y}+\mathbf{x} \in \bar{L}) \Longrightarrow(\mathrm{X} \in \overline{\mathscr{L}})$

Voici maintenant d'autres systèmes l'axiomes équivalents : 
Théorème 3.- Soit $r$ un rang sur $E$ et $\mathscr{C}$ l'ensemble des r-circuits, les propriétés suivantes sont équivalentes :

( $R$ R) $\quad r$ est un rang régulier

$\left(\mathrm{c}_{2}\right) \quad \forall \mathrm{x} \in \mathscr{C}, \forall \mathrm{Y} \in \mathscr{C}, \mathrm{X} \neq \mathrm{Y}, \forall \mathrm{x} \in \mathrm{X} \cap \mathrm{Y}, \exists \mathrm{z} \in \mathscr{\zeta}$ tel que $z c \mathrm{X} \cup \mathrm{Y}-\{\mathrm{x}\}$

$\left(c^{\prime}{ }_{2}\right) \quad \forall \mathrm{X} \in \mathscr{C}, \forall \mathrm{Y} \in \mathscr{C}, \forall \mathrm{a} \in \mathrm{X} \cap \mathrm{Y}, \forall \mathrm{x} \in \mathrm{X}-\mathrm{Y}, \exists Z \in \mathscr{C}$ tel que $\mathrm{x} \in Z$ et $Z \subset \mathrm{X} \cup \mathrm{Y}-\{\mathrm{A}$ $\left(C_{2}\right)$ Toute partie $X \in \mathcal{f}(E)$ telle que $r(X)=|X|-1$ contient un circuit et un seul

\section{Démonstration}

On vérifie comme ci-dessus : $(R R) \Longrightarrow\left(C_{2}\right) \Longrightarrow\left(C^{\prime}{ }_{2}\right) \Longrightarrow(R R)$ et $\left(C_{2}\right) \longleftrightarrow\left(C_{2}\right)$. Les seuls points délicats sont :

$\left.1^{\circ}\right)\left(C{ }_{2}\right) \longleftrightarrow(R R) \quad$ : Soit $E$ vérifiant $\left(C{ }_{2}\right), F \in F(E)$ est dite régulière si les parties libres maximales incluses dans $F$ ont toutes même cardinal. Si toute $F \in \mathcal{F}(E)$ est régulière, $r$ est régulier. Nous allons le démontrer par récurrence sur $|F|$. C'est trivial pour $|F|=0$ et supposons le vrai pour $|F| \leqslant n-1$. Soit $F \in \mathcal{F}(E)$ avec $|F|=n$

$$
\begin{gathered}
\text { Si } r(F)=n \quad F \text { est libre, donc régulière } \\
\text { Si } r(F)=n-1 \quad F \text { contient un seul circuit, donc est régulière } \\
\text { Si } r(F) \leq n-2, \quad \text { soit } Y \text { une partie libre maximale incluse dans } F \text {. } \\
\forall x \in F-Y \quad \text { tel que }\{x\} \in \mathscr{L}, \quad Y+x \text { est lié, donc (Y+x régulière), } \\
\exists y \in Y \text {, } Y-y+x \in \mathcal{L} \text {. } Y-y+x \text { est libre maximale car : } \\
\text { ou bien }|Y|=|Y-y+x|=n-2 \quad \text { et } r(F)=n-2 \\
\text { ou bien }|Y|<n-2 \quad \text { et si } Y-y+x+z \supsetneqq Y-y+x \text { et est libre, }
\end{gathered}
$$
on contredit le fait que $Y+x+z$ soit régulière.

Alors $Y$ et $Z$ étant deux parties libres maximales de $F$, on peut passer de $Y$ à $Z$ en échangeant les éléments un par un, d'où $|Y|=|Z|$, et $F$ régulière.

20) $\left(\mathrm{C}_{2}\right) \Longrightarrow\left(\mathrm{C}_{2}{ }_{2}\right):$ Nous allons démontrer par récurrence sur $|\mathrm{X} \cup \mathrm{Y}|$ que $\forall \mathbf{X} \in \mathbf{X} \cap \mathbf{Y}, \forall a \in X-Y, \exists Z \in \mathscr{C}, Z \subset X \cup Y-\{x\} \quad$ et $a \in Z$, si $X \in \mathcal{C}$ et $Y \in \ell$. C'est trivial si $|X \cup Y|=0,1$ ou 2 . Supposons le vrai si $|X \cup Y| \leqslant n-1$ et soit $\mathrm{X} \in \mathscr{C}, \mathrm{Y} \in \mathscr{C}$ te]s que $|\mathrm{X} \cup \mathrm{Y}|=\mathrm{n}, \quad a \in \mathrm{X} \cap \mathrm{Y} \quad$ et $\mathrm{X} \in \mathrm{X}-\mathrm{Y}$. 
S'il existe $z \in \mathscr{C}, \quad Z \subset X \cup Y-\{x\}$ tel que $|Z \cup X|<n$, il suffit de raisonner sur $X$ et $Z$ et d'utiliser 1 'hypothèse de récurrence.

Sinon, tout $Z \in \mathscr{C}$ tel que $Z \subset X \cup Y-\{X\}$ vérifie : $Z \supset Y-X$. Alors, d'après $\left(C_{2}\right)$ il existe au moins un tel $z$, et $\forall t \in Y-X$, il existe $T \in \mathscr{C}$, $T \subset Y \cup Z-\{t\}$

ou bien $x \in Z$

ou bien $x \notin z$, alors $T \neq X,|X \cup T|<n$ et on a $Z \prime \subset X \cup T-\{a\}$ et $\mathbf{X} \in \mathbf{Z}$ ' dans $X \cup T$

Théorème 4.- Soit $r$ un rang sur $E$ et $B$ l'ensemble des bases. $r$ est régulier si et seulement si $B$ vérifie les axiơnes $\left(B_{1}\right),\left(B_{2}\right)$ et :

$\left(B_{3}\right) \quad \forall B \in B, \forall B^{\prime} \in \mathcal{B}, \forall x \in B^{\prime}-B$, les seules bases contenant $x$ et incluses lans $B+x$ sont de la forme $B-y+x$ où $y \in B_{x}$ partie finie non vide de $B$.

Alors toutes les bases de $\mathbf{E}$ ont meme cardinal, et ce cardinal est appellé la dimension de $E$ : le rang infini est lui-même régulier.

Si $r$ est un rang régulier, pour toute base $B$, pour tout $x \in E$ tel que $\{x\} \in \mathcal{L}$ (donc $x \in B^{\prime}$ pour une base $B^{\prime}$ au moins);

ou bien $x \in B$ et $B_{x}=\{x\} \quad$ ( $l$ 'échange doit être effectif).

ou bien $x \notin B$ et, d'après $\left(C_{2}^{\prime}\right)$ et $\left(C_{2}{ }_{2}\right), B+x$ contient un circuit et un seul $C$, et $B-y+x \in B$ si et seulement $s i \quad y \in B=B \cap C$.

Réciproquement soit $r$ un rang tel que $B$ vérifie les axiomes $\left(B_{1}\right),\left(B_{2}\right)$ et $\left(B_{3}\right)$. Soient $F \in \mathcal{F}(E)$, $X$ et $Y$ parties libres maximales incluses dans $F$, et $Y-X=\left\{y_{1}, \ldots, y_{m}\right\}$. Il existe $B^{0} \in B^{3}$ tel que $X \subset B^{0}$ et $B^{\prime} \in B$ tel que $Y \subset B^{\prime}$

Comme $\mathrm{X}+\mathrm{y}_{1}$ est liée, $\mathrm{B}_{\mathrm{y}_{1}} \cap(\mathrm{X}-\mathrm{Y}) \neq \varnothing$. Soit $\mathrm{x}_{1} \in \mathrm{B}^{\circ} \mathrm{y}_{1} \cap(\mathrm{X}-\mathrm{Y})$ $B^{1}=B^{0}-x_{1}+y_{1} \in B$ et il suffit d'itérer ceci avec $x^{1}=x-x_{1}+y_{1}$ et $\mathbf{Y}^{1}=\mathrm{Y}$. Après $\mathrm{m}$ itérations, on $\mathrm{a}: \mathrm{Y}^{\mathrm{m}}=\mathrm{Y}, \mathrm{X}^{\mathrm{m}}=\mathrm{X}-\left\{\mathrm{x}_{1}, \ldots, \mathrm{x}_{\mathrm{m}}\right\}+(\mathrm{Y}-\mathrm{X})$ où les $x_{i}$ sont tous différents, et $B^{m}=B^{0}-\left\{x_{1}, \ldots, x_{m}\right\}+(Y-X) \in B$ or $\quad B^{m} \supset X^{m}$, et $Y$ étant libre maximale dans $F, \quad X^{m}=Y:|X|=|Y|$.

Soient B et B' deux parties libres maximales incluses dans F. Si B 
est fini, $B^{\prime} l^{\prime}$ 'est aussi et $|B|=\left|B^{\prime}\right|$ (raisonner sur des parties finies contenant $B)$; si $B$ est infini, alors $\left(B_{x}\right)_{x \in B}$, est un recouvrement de $B$ : en effet pour tout $y \in B$,

$$
\begin{aligned}
& \text { ou bien } y \in B^{\prime} \quad \text { et } \quad B_{y}=\{y\} \\
& \text { ou bien } y \notin B^{\prime} \quad \text { et } \quad B^{\prime}+y \text { est lié. L'ensemble des circuits }
\end{aligned}
$$

inclus dans $B \cup B^{\prime}$ et contenant $y$ est non vide et ceux de ces circuits tels que $|C \cap B|$ soit minimal, vérifie $\left|C \cap B^{\prime}\right|=1$. Donc $C \cap B^{\prime}=\{t\}$ et $C=B_{t}+t$ contient $y: y \in B_{t} \cdot$ Alors $^{(*)}$ :

$$
|B| \leq \sum\left|B_{x}\right| \quad\left[x \in B^{\prime}\right]=\left|B^{\prime}\right|
$$

et inversant les rôles de $B^{\text {et }} B^{\prime},\left|B^{\prime}\right|=\left|B^{\prime}\right|$.

\section{3.- Fonctions génératrices et parties libres}

\subsection{1.- Fonctions génératrices minces}

Soit $E$ un $\mathscr{L}$-espace. L'application $\varphi$ (ou mieux $\varphi_{\mathscr{L}}$ ) de $\dot{\beta}(E)$ dans lui-même, qui à tout $x \in \mathcal{\beta}$ (E) associe :

$$
\varphi_{\mathscr{L}}(\mathrm{X})=\varphi(X)=\{\mathbf{x} \mid \mathbf{x} \in X \text { ou } \mathbf{x} \in \mathbf{E} \text { et } \exists \mathbf{Y} \in \mathscr{L}, \mathbf{Y} \subset \mathrm{X} \text { et } \mathrm{Y}+\mathrm{X} \notin \mathscr{L}\}
$$

vérifie les propriétés :

$\left(G_{1}\right) \quad$ (caractère fini) $: \forall x \in \mathcal{F}(E), \varphi(X)=U_{\varphi(Y)}[y \in \mathcal{F}(\mathrm{X})]$

$\left(\mathrm{G}_{2}\right) \quad \forall \mathrm{x} \in \mathcal{\beta}(\mathrm{E}), \mathrm{x} \subset \varphi(\mathrm{x})$

(G) (échange) $\quad \forall x \in \ngtr(E), \forall x \in E, \forall y \in E$ tels que $y \notin \varphi(x)$, si $y \in \varphi(x+x)$, alors $x \in \varphi(x+y)$

Mais en général $\varphi$ n'est pas une fermeture (i.e. ne vérifie pas $\varphi \circ \varphi=\varphi)$. L'on se propose maintenant de définir les parties libres à partir des fonctions génératrices.

(*) Un résultat classique de la théorie des cardinaux infinis dit que si $\left(a_{i}\right)_{i \in I}$ est une famille de cardinaux telle que : il existe un cardinal infini a pour lequel : $\forall i \in I, \quad 1 \leqslant a_{i} \leqslant a \quad$ et $|I| \geqslant a$, alors $\sum a_{i}[i \in I]=|I|$ 
Définition 5.- L'on appelle fonction génératrice mince (en abrégé : $\underline{\mathrm{f}} \mathrm{g}$ ) d'un ensemble $E$ dans un ensemble $F$ (resp. sur un ensemble $E$ ) toute application $\varphi$ de $\beta(E)$ dans $\beta(F)$ (resp. dans lui-mème) vérifiant l'axiome $(G)$.

En particulier toute $f \mathrm{~g} \mathrm{~m}$ est croissante.

exemples : 1) Soit $M$ un A-module à gauche, $\varphi(X)$ l'ensemble des combinaisons linéaires d'éléments de $X . \varphi$ est une $f \mathrm{gm}$ sur $M$

qui vérifie $\left(\mathrm{G}_{2}\right)$ si $M$ est un module unitaire qui vérifie $\left(G_{2}\right)$ et $\left(G_{3}\right)$ si $M$ est un espace vectoriel sur le corps $A$.

2) Soit - une loi de composition interne sur $E$ et $A C E$. $\varphi(X)=A X \quad($ resp. $\varphi(X)=X . X)$ est une $f g$ m sur $E$.

3) Soit $\phi$ une correspondance de E dans F $\quad X \longrightarrow \phi(X)$ est une $f \mathrm{~g} m$ de $E$ dans $F$, en particulier quand $\oint$ est le graphe d'une application.

Voici en outre la :

Proposition2 : Tout produit fini de $f \mathrm{~g} \mathrm{~m}$ est une $\mathrm{f} \mathrm{g}$.

\subsubsection{Parties et familles exactement libres d'une $f \mathrm{~g} \mathrm{~m}$.}

Définition 6.- Soit $\varphi$ une $f \mathrm{~g} m$ de $E$ dans $F$ et $A C F$. On dit que $X$ $\varphi$-engendre $A$; ou encore que $X$ est un système générateur de $A$ si $\varphi(X)=A$. En outre une partie $X$ de $F$ est dite exactement libre si $X$ est un système générateur minimal de $\varphi(X)$. Sinon $X$ est dite exactement liée. $X$ est une base de A si $\varphi(X)=A$ et $X$ est exact ement libre.

Une famille $\left(x_{i}\right)_{i \in I}$ d'éléments de $E \quad \varphi$-engendre A si $A=\varphi\left(\left\{x_{i} \mid i \in I\right\}\right)$ elle est exactement libre $s$ 'il $n$ 'existe pas de sous-famille stricte qui engendre $\varphi\left(\left\{x_{i} \mid i \in I\right\}\right) ;$ sinon elle est exactement liée.

$$
\begin{aligned}
& \text { L'ensemble } \mathcal{L}_{\varphi}^{\mathbf{e}} \text { des parties exactement libres est : } \\
& \mathscr{L}_{\varphi}^{\mathbf{e}}=\{\mathrm{x} \mid C \mathrm{CE} \text { et } \forall \mathbf{x} \in \mathrm{X}, \quad \varphi(\mathrm{x}-\mathrm{x}) \underset{\ddagger}{\subsetneq} \varphi(\mathrm{x})\}
\end{aligned}
$$


et $\left(x_{i}\right)_{i \in I}$ est exactement libre si et seulement si $\left\{x_{i} \mid i \in I\right\}$ l'est et les $x_{i}$ sont distincts deux à deux.

$$
\begin{aligned}
& \varphi \text { est "caractérisée" par ses "valeurs" sur } \mathscr{L}_{\varphi}^{\mathrm{e}} \cap \mathcal{F}(\mathrm{E}) \text { : } \\
& \forall \mathrm{X} \in \mathcal{F}(\mathrm{E}), \quad \varphi(\mathrm{X})=\cup \varphi(\mathrm{Y}) \quad\left[\mathrm{Y} \in \mathcal{F}(\mathrm{X}) \cap \mathscr{L}_{\varphi}^{\mathrm{e}}\right]
\end{aligned}
$$
on a :

$\mathscr{L}_{\varphi}^{e} n^{\text {'est }}$ ni de caractère fini, ni même inductif en général. Cependant

$$
\emptyset \in \mathscr{L}_{\varphi}^{\mathrm{e}} \quad \text { et } \quad \forall \mathrm{X} \in \mathscr{L}_{\varphi}^{\mathrm{e}} \quad, U \mathrm{Y} \quad\left[\mathrm{Y} \in \mathbb{F}^{\mathrm{f}}(\mathrm{x}) \cap \mathscr{L}_{\varphi}^{\mathrm{e}}\right]=\mathrm{x}
$$

exemple.- Soit $E$ un ensemble, $\mathscr{L} \subset F^{\prime}(E)$ et $\emptyset \in \mathscr{L}$. Alors pour tout $X \subset E$ : $\forall x \in Z(E), \varphi(x)=\operatorname{Sup} n-1\left[n \in \omega_{0}\right.$ et $\exists\left(x_{i}\right)_{i \in n} \quad$ strictement croissante

$$
\text { telle que } \left.\forall i \in n, x_{i} \in \mathscr{F}(x) \cap \mathscr{L}\right]
$$

est une $f \mathrm{~g} m$ de $E$ dans $\omega_{0}+1$ et l'on a :

$$
\mathscr{L}_{4}^{\mathrm{e}} \cap \mathscr{I}^{\prime}(\mathrm{E})=\mathscr{L}
$$

Fn général $\mathscr{L}_{\varphi}^{e} n^{\prime}$ est pas inductif. Il l'est cependant si et seulement si: il n'existe pas de familles $\left(x_{n}^{m}\right)_{n \in \omega_{0}}^{m \in \omega_{0}}$ d'éléments de $\mathscr{L}$ telles que : $\forall m \in \omega_{0}, \quad\left(x_{n}^{m}\right)_{n \in \omega_{0}} \quad$ est strictement croissante à partir d'un certain rang,

$$
\text { et } \cup x_{n}^{m} \quad\left[n \in \omega_{0}\right] \supsetneqq \quad \cup \quad x_{n}^{m+1} \quad\left[n \in \omega_{0}\right]
$$

Alors dans ce cas :

$$
\mathscr{L}_{\varphi}^{\mathbf{e}}=\{\mathrm{x} \mid \mathrm{x} \subset \mathrm{E} \quad \text { et } \quad \forall \mathrm{Y} \in \mathcal{H}(\mathrm{x}), \exists \mathrm{z} \in \mathfrak{F}(\mathrm{x}) \cap \mathscr{L}, \quad \mathrm{z} \supset \mathrm{Y}\}
$$

4 n'est pas un rang faible en général.

\subsubsection{Diagonalisation d'une f.g.m.}

Il est bon de donner à tous les ensembles considérés un élément nul universel 0 et de supposer que $\varphi(\phi) \supset 0$ pour toute $f \mathrm{~g} \mathrm{~m} \varphi$.

Soit $\varphi$ une $f \mathrm{~g} m$ de $E$ dans $F$ et $\Phi$ une correspondance de $E$ dans $F$. $\mathcal{L}_{\varphi}^{\Phi}=\{\mathbf{X} \mid \forall \mathbf{x} \in \mathbf{X}, \Phi(\mathbf{x}) \cap \varphi(\mathbf{x}) \neq \varnothing$ et $\Phi(\mathbf{x}) \cap(\varphi(\mathbf{X})-\varphi(\mathbf{X}-\mathbf{x})) \neq \varnothing\}$ 
vérifie

$\mathscr{L}_{\varphi}^{\Phi} \subset \mathscr{L}_{\varphi}^{\mathbf{e}}$

Si l'on prend $\Phi=\{(\mathrm{x}, \mathrm{y}) \mid \mathrm{x} \in \mathrm{E} \quad$ et $\mathrm{y} \in U(\varphi(\mathrm{X})-\varphi(\mathrm{X}-\mathrm{x})) \quad[\mathrm{X} \subset \mathrm{E}]\}$, on a $\mathscr{L}_{\varphi}^{\phi}=\mathscr{L}_{\varphi}^{\mathrm{e}}$

Si $\Phi$ est une application de $E$ dans $F$, alors $\mathscr{L}_{\varphi}^{\Phi}$ est de caractère fini.

On appelle diagonale de $\varphi$ toute application $\sigma$ de $E$ dans $F$ telle que $\mathscr{L}_{\varphi}^{\sigma}=\mathscr{L}_{\varphi}^{\mathrm{e}}$ et $\sigma(\mathbf{x})=0$ pour tout $\mathbf{x} \in \mathrm{E}$ tel que $\varphi(\mathbf{x})=\varphi(\phi)$. Une $\mathbf{f} \mathbf{g}$ $\varphi$ est dite diagonalisable si elle admet une diagonale.

or $\varphi$ est diagonalisable si et seulement si :

$$
\forall \mathbf{x} \in \mathbf{E}, \quad \mathbf{F}_{\mathbf{X}}=n(\varphi(\mathbf{Y})-\varphi(\mathbf{Y}-\mathbf{x}))\left[\mathbf{X} \in \mathscr{L}_{\varphi}^{\mathbf{e}}, \mathbf{Y} \in \mathcal{F}^{(X)} \text { et } \mathbf{x} \in \mathbf{Y}\right] \neq \varnothing
$$

en particulier si $\varphi(x)=\varphi(\phi)$, pour tout $x \in \mathscr{L}_{\varphi}^{\mathbf{e}}, \mathbf{x} \notin X$. On pose alors $F_{x}=0$. Toute application $\sigma$ de $E$ dans $F$ telle que $\sigma(x) \in F_{x}$ pour tout $x \in E$ est une diagonale pour $\varphi$, et on les a toutes ainsi. En outre dans ce cas :

$\psi=\sigma^{-1} \circ \varphi$ est une $\mathrm{f} \mathrm{g} \mathrm{m}$ sur $\mathrm{E}$ qui vérifie $\left(\mathrm{G}_{1}\right)$.

On a $\mathscr{L}_{\psi}^{\mathrm{e}} \subset \mathscr{L}_{\varphi}^{\mathrm{e}}$, et si $\sigma$ est injective pour $\{\mathbf{x} \mid \sigma(\mathbf{x}) \neq 0\}$, $\mathscr{L}_{\psi}^{\mathbf{e}}=\mathscr{L}_{\varphi}^{\mathrm{e}}=\mathscr{L}_{\psi}^{1} \mathbf{E}$ est de caractère fini, $1_{\mathrm{E}}$ est une diagonale pour $\psi$ et l'on a :

$$
\mathscr{L}_{\psi}^{e}=\{x \mid x \in E \quad \text { et } \forall x \in X \quad, \quad x \notin \Psi(x-x)\}=\mathscr{L}_{\varphi}^{e}
$$

et

$\forall \mathrm{X} \subset \mathrm{E}, \Psi(\mathrm{X}) \subset\left\{\mathrm{x} \mid \mathrm{x} \in \mathrm{X}\right.$ ou $\mathrm{X} \in \mathrm{E}$ et $\left.\exists \mathrm{Y} \in \mathscr{F}(\mathrm{X}) \cap \mathscr{L}_{\varphi}^{\mathrm{e}}, \mathrm{Y}+\mathrm{x} \notin \mathscr{L}_{\varphi}^{\mathrm{e}}\right\}$

l'inclusion étant stricte en général, sauf si $\psi$ vérifie $\left(G_{3}\right)$.

Dans ce dernier cas, on dit que $\varphi$ est injectivement diagonalisable.

Théorème 50- Soit $\varphi$ une $f \mathrm{~g} \mathrm{~m}$ de $E$ dans $F$ telle que $\varphi(x)-\varphi(\varnothing)$ soit fini pour tout $x \in E$. Alors $\varphi$ est diagonalisable (resp. injectivement) si et seulemert si il existe un ensemble $\mathscr{F}_{C} \mathscr{F}(E)$ et cofinal à $\mathscr{F}(E)$ telle que :

$$
\forall x \in f,\left.\varphi\right|_{x}: \not(x) \longrightarrow \xi(F) \quad \text { (restriction de } \varphi \text { ) }
$$

soit diagonalisable (resp. injectivement). 
La démonstration se fait en utilisant la limite projective : Soit pour tout $X \in F(E), \quad \Sigma_{X}$ l'ensemble des diagonales de $\varphi / X \cdot$ Si $X \subset Y$ et $Y \in \mathscr{F}_{(E)}, u_{X Y}: \Sigma_{Y} \longrightarrow \Sigma_{X}$ où $u_{X Y}(\sigma)=\left.\sigma\right|_{X}$ $\left(\Sigma_{X}, u_{X Y}\right) \quad\left[X, Y \in \Psi^{\prime}(E)\right]$ est un système projectif d'ensembles finis et non vides, donc est non vide (voir $[\mathrm{C}, \mathrm{I}, 3], \oint 7, \mathrm{n}^{\circ} 4$ par exemple), et alors toute $\sigma \in$ lim. $_{.}\left(\Sigma_{X}, u_{X Y}\right)$ est diagonale de $(E, \varphi, F)$. D'où le théorème 5 sachant que :

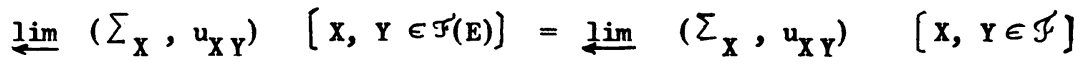

On fait le même raisonnement pour les diagonales injectives.

\subsection{4.- Fonctions génératrices.}

Outre les propriétés $\left(G_{1}\right),\left(G_{2}\right)$ et $\left(G_{3}\right)$ de 1.3.1., une application $\varphi$ de $\beta(E)$ dans $\beta(E)$ peut vérifier les propriétés :

$\left(G_{4}\right) 1_{E}$ est une diagonale pour $\varphi$, i.e. $\mathscr{L}_{\varphi}^{1_{E}} \supset \mathscr{L}_{\varphi}^{e}$

$\left(G_{5}\right) \quad \forall x \in f(E)-\mathscr{L}_{\varphi}^{1} E \quad, \varphi(x)=U \varphi(X-x) \quad[x \in X]$

$\left(\mathrm{G}_{6}\right) \quad$ (fermeture) $\varphi \circ \varphi=\varphi$

Définition 7.- On appelle fonction génératrice faible (en abrégé : $f \mathrm{~g} f$ ) sur un ensemble $E$ toute application $\varphi$ de $\$(E)$ dans lui-même qui vérifie les axiomes $\left(G_{1}\right)$ et $\left(G_{2}\right)$. Une $f \mathrm{~g} f$ est dite échangiste (resp. diagonale ; resp. exacte) si elle vérifie l'axiome $\left(G_{3}\right)\left(\right.$ resp. $\left(G_{4}\right)$; resp. $\left(G_{5}\right)$ ) • Une fermeture sur $E$ est une $f \mathrm{~g} f$ qui vérifie $l^{\text {taxiome }}\left(G_{6}\right)$.

L'on désigne par $\Phi^{f=}$ la catégorie dont les objets sont les couples $(E, \varphi)$ où $\varphi$ est une $f \mathrm{~g} f$ sur $E$, et dont les morphismes de $(E, \varphi)$ dans $\left(E^{\prime}, \varphi^{\prime}\right)$ sont les applications $f$ de $E$ dans $E^{\prime}$ telles que :

$$
\left(H_{1}\right) \quad f \circ \varphi=\varphi^{\prime} \circ f
$$

F : $\quad(E, \varphi) \longrightarrow\left(E, \mathscr{L}_{\varphi}\right)$ où

(4) $\mathscr{L}_{\varphi}=\{x \mid x \subset E$ et $\forall x \in X, \quad x \notin \varphi(x-x)\}=\mathscr{L}_{\varphi}^{1} E$

est un foncteur ensembliste de $\Phi^{f=}$ dans $L_{\text {. }}$ 
D'où la sur-catégorie propre ${ }^{(*)}$ de $\Phi^{f=}: \Phi^{f}$ où les morphismes de $(E, \varphi)$ dans $\left(E^{\prime}, \varphi^{\prime}\right)$ sont les applications $u$ de $E$ dans $E^{\prime}$, morphismes de $\left(E, \mathscr{L}_{\varphi}\right)$ dans $\left(E, \mathscr{L}^{\prime}{ }_{\varphi}\right)$. F s'étend alors en un foncteur ensembliste de $\Phi_{m}^{f}$ dans $\underset{m}{L}$ qui sera aussi noté $F$.

G : $(E, \mathscr{L}) \longrightarrow\left(E, \varphi_{\mathscr{L}}\right) \quad$ où $\varphi_{\mathscr{L}}$ est défini par (3) est un foncteur ensembliste de $\underset{m}{L}$ dans $\underset{\Phi^{f}}{\Phi^{f}}$. Soit $G(L)=\underset{m}{g}$

F et $\mathrm{G}$ induisent sur $\underset{\mathrm{L}}{\mathrm{L}}$ et $\Phi^{\mathrm{g}}$ des isomorphismes inverses l'un de l'autre : L'on écrit $\underset{m}{L}=F\left(\Phi^{f=}\right)=F\left(\Phi_{m}^{\Phi}\right)$.

Définition 7'.- On appelle fonction génératrice sur un ensemble $E$ toute fonction génératrice faible $\varphi$ sur $\mathrm{E}$ vérifiant l'une des propriétés équivalentes cidessous :

1) Il existe $\mathscr{L} \subset \bar{\beta}(\mathrm{E})$, de caractère fini telle que $\varphi_{\mathscr{L}}=\varphi$ (voir (3))

2) $\varphi_{\mathscr{L}_{\varphi}}=\varphi \quad$ (voir (3) et (4))

3) $\varphi$ est échangiste et exacte

4) : $\forall \mathbf{X} \subset \mathbf{E}, \varphi(\mathrm{X})=U \varphi(\mathrm{Y}) \quad\left[\mathrm{Y} \in \oiint_{(}(\mathrm{X}) \cap \mathscr{L}_{\varphi}\right]$, et $\varphi$ est échangiste.

La structure d'ensemble muni d'une fonction génératrice est donc équivalente à celle de $\mathscr{L}$-espace. Voici quelques relations liant $\varphi, \mathscr{L}, \ldots$

$$
\begin{aligned}
& \forall \dot{X} \subset \mathrm{E}, \quad \varphi_{\mathscr{C}}(\mathrm{x})=\mathrm{X} \cup\left\{\mathrm{x} \mid \mathrm{x} \in \mathrm{E} \text { et } \exists \mathrm{Y} \in \mathcal{F}^{\prime}(\mathrm{x}), \mathrm{Y}+\mathrm{x} \in \mathcal{C}\right\} \\
& \text { ou } \varphi_{\mathbf{r}}(\mathrm{X})=\{\mathbf{x} \mid \mathbf{X} \in \mathbf{E} \text { et } \exists \mathrm{Y} \in \mathcal{F}(\mathrm{X}), \mathbf{r}(\mathrm{Y}+\mathbf{x})=\mathbf{r}(\mathrm{Y})\} \\
& (\forall \mathrm{x} \in \mathscr{L}, \forall \mathrm{x} \in \mathrm{E}-\mathrm{X}) \quad\left(( \mathrm { x } + \mathrm { x } \in \mathcal { L } ) \Longleftrightarrow \left(\mathrm{x} \notin \varphi_{\left.\mathcal{L}^{(x)}\right)}\right.\right.
\end{aligned}
$$

(*) La terminologie employée pour les catégories est:

1) $\subseteq$ sous-catégorie propre de $\varsigma^{\prime}$ si et seulement si $\subseteq$ et $\varsigma^{\prime}$ ont les memes objets.

2) $\subseteq$ sous-catégorie pleine de $S^{\prime}$ si et seulement si pour tout couple $(A, B)$ d'objets de $\subseteq, \quad \leq(A, B)=\leqslant$, $(A, B)$. 
En particulier, $X \subset E$ est libre si et seulement si il existe un ordre total sur $X$ tel que, pour tout $x \in X, \quad x \in \varphi(] \leftarrow, x[)$. Alors cette propriété est vraie pour tous les ordres totaux.

La planche I donne les équivalences complètes.

1.4.- Foncteurs de fermeture. Régularisation. Treillis des sous-espaces. $\varphi$-espaces

$\underset{m}{\Phi^{f}}<\left(\right.$ resp. $\underset{\phi_{m r}}{f=}$ ) est la catégorie dont les objets sont les couples $(E, \varphi)$ où $\varphi$ est $f \mathrm{~g} f$ sur $E$, les morphismes de $(E, \varphi)$ dans $(F, \psi)$ étant les applications $f$ de $E$ dans $F$ telles que :

$$
\left(H_{1}^{<}\right) \quad f \circ \varphi \subset \psi \circ f \quad\left(\operatorname{resp} .\left(H_{1}\right) \quad f \circ \varphi=\psi \circ f\right)
$$

$\underset{m}{\Phi<}$ (resp. $\Phi^{\Phi=}$ ) en est la sous-catégorie pleine "des fonctions génératrices",
$\underset{\mathrm{m}}{\mathrm{F}}<($ resp. $\underset{\mathrm{m}}{\mathrm{F}}=$ ) en est la sous-catégorie pleine "des fermetures",
et $\underset{m}{\Phi} \quad$ (resp. $\underset{m}{\Phi}$ ) en est la sous-catégorie pleine "des fonctions généra- trices régulières".
$\underset{m}{\mathrm{~F}}$ (resp. $\underset{m}{\Phi}$ ) est la sous-catégorie pleine de $\underset{m}{\Phi^{\mathbf{f}}} \operatorname{dont}$ les objets sont "les fermetures" (resp. "les fonctions génératrices régulières")

\subsection{1.- Le foncteur de fermeture. $\varphi$-espaces .}

$$
P:(E, \varphi) \longrightarrow\left(E, \omega_{\varphi}\right) \text { où } \quad \omega_{\varphi}=U_{\varphi}{ }^{n} \quad\left[n \in \omega_{0}\right]
$$

(pour tout entier $n \geqslant 1, \varphi^{n}$ est la $n$-ième itérée de $\varphi$ )

est un foncteur ensembliste de $\Phi^{f}$ sur $F^{<}$tel que $P$ o $P=P$

$P$ projette $\underset{m}{\Phi} f=$ sur $\underset{m}{F}=$ et se réduit à l'identité sur $\underset{m}{F^{<}}$.

$\omega_{\varphi}$ est la fermeture la moins $\Phi_{m}^{f^{<}}$-fine sur $E$ telle que $\varphi \subset \omega_{\varphi}$.

P est le foncteur de fermeture. 
Renarque $P n^{\prime}$ 'est plus un foncteur ensembliste de $\underset{\Phi^{f}}{\mathbf{s u r}} \underset{\mathrm{Nm}}{\mathrm{F}}$

Toute fermeture $\varphi$ est une $f$ g diagonale, mais $n$ 'est pas échangiste en général. Si elle est échangiste, alors $\zeta_{\varphi}$ est régulier (vérifier $\left(c_{2}\right)$ par exemple). On a :

$$
\underset{m}{\Phi}=\underset{m}{\mathbf{F}} \cap \underset{m}{\Phi}
$$

et on pose la:

Definition 8.- On appelle $\varphi$-espace; tout couple $(E, \varphi)$ où $\varphi$ est une fonction génératrice régulière sur $\mathrm{E}$ (i.e. $\mathcal{L}_{\varphi}$ est régulière).

Soit $\varphi$ une application de $\xi(E)$ dans lui-meme. $(E, \varphi)$ est un $\varphi$-espece si et seulement si $\varphi$ vérifie les axiomes $\left(G_{1}\right),\left(G_{2}\right),\left(G_{3}\right)$ et $\left(G_{6}\right)$, i.e. si et seulement si $\varphi$ est une fermeture échangiste.

\subsection{2.- Régularisation d'un $\mathscr{L}$-espace. Cloture}

Mére si $(E, \varphi)$ est une fonction génératrice, $\left(E, \omega_{\varphi}\right)$ n'est pas en général un $\varphi$-espace. L'on va cépendant associer canoniquement à toute fgf $\varphi$ sur $E$ une fg régulière $\varphi R$ sur $E$ ainsi :

$$
\begin{aligned}
& \text { Soit } \varphi_{0}=\varphi \\
& \varphi_{1}=\omega_{\varphi_{0}} \quad, \varphi_{2}=\varphi_{\mathscr{L}} \varphi_{1} \\
& \text {.... } \quad \cdots \\
& \text { et pour tout entier } n \geqslant 0, \quad \varphi_{2 n+1}=\omega_{\varphi 2 n}, \varphi_{2 n+2}=\varphi_{2 \varphi_{2 n+1}} \\
& \text { et } \varphi R=U \varphi_{n} \quad\left[n \in \omega_{0}\right] \\
& \text { où } \mathscr{L}_{\varphi}=\{x / x \in E \text { et } \forall x \in X, \quad x \notin \varphi(x-x)\} \\
& \text { et, } \forall x \in \beta(E), \varphi \mathscr{L}(x)=x \cup\left\{x / x \in E \text { et } \exists y \in F^{\mathscr{T}}(x), \quad y \in \mathcal{L} \text { et } y+x \notin \mathscr{L}\right\} \\
& \varphi R \text { est la fonction génératrice régularisée de } \varphi
\end{aligned}
$$




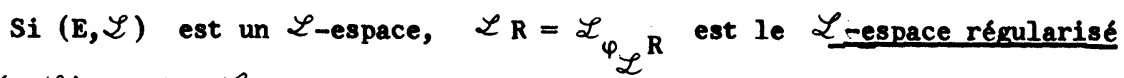
de $(E, \mathscr{L})$, d'où $\mathscr{C}_{\mathbf{R}} \ldots$

Pour tout $X \subset E, \varphi R(X)$ est la $\varphi$-cloture de $X$

et $\omega_{\varphi}(\mathrm{X})$ est la fermeture de $\mathrm{X}$

L'on a : $\omega_{\varphi} \subset \varphi R$ et $\omega_{\varphi} R=\varphi R$.

\section{Remarques}

$\left.1^{\circ}\right)(E, \varphi) \longrightarrow(E, \varphi R)$ ne définit pas un foncteur ensembliste de $\Phi_{m}^{f^{<}}$

(resp. $\Phi_{m}^{f=}$ ) dans $\Phi_{m}^{<}$(resp. dans $\underset{. m}{\Phi}$ ).

$\left.2^{\circ}\right) \varphi R \quad n$ 'est pas en général la structure de $\varphi$-espace la moins $\Phi^{f<}$-fine sur E telle que $\varphi \subset \varphi R$. Plus précisément, si $\psi$ est une structure de $\varphi$-espace telle que $\varphi \subset \psi$, en général $\varphi R \notin \psi$

$\left.3^{\circ}\right)$ Si $\varphi \subset \varphi^{\prime} \quad$ (resp. si $\mathscr{L}_{\varphi^{\prime}} \subset \mathscr{L}_{\varphi}$ ) en général $\varphi R$ et $\varphi^{\prime} R$ ne sont pas comparables dans $\Phi_{m}^{<}(E)$ (resp. dans $\Phi_{m}(E)$ ).

\subsubsection{Ensembles de sous-espaces et fermetures}

Soit $\varphi$ une $f g$ sur $E$. L'on appelle sous-espace de $E$ tout couple $\left(A /\left.A\right|_{A}\right)$ où $A \subset E$ et où $\left.\varphi\right|_{A}$, restriction de $\varphi$ à $\beta(A)$, est une application de $\beta$ (A) dans Iui même.

L'ensemble $\mathscr{S}=\mathrm{T}(E, \varphi)$ des sous-espaces est :

$$
T(E, \varphi)=\{A / A \subset E \text { et } A=\varphi(A)\}
$$

et vérifie :

$$
T(E, \varphi)=T\left(E, \omega_{\varphi}\right)=\left\{\omega_{\varphi}(\mathbf{X}) / \mathbf{X} \subset E\right\}=\mathscr{Y}
$$

$\left(s_{1}\right) E \in \mathcal{J}$ et toute intersection d'éléments de $\mathcal{J}$ appartient à $\mathcal{J}$

$\left(\mathrm{s}_{2}\right) \quad \mathcal{Y}$ est inductif pour l'inclusion 
$\mathcal{Y}$ est un treillis complet inductif pour l'inclusion et l'on a :

$$
\wedge A_{i}=\cap A_{i} \quad \text { et } \quad \vee A_{i}=\omega_{\varphi}\left(U A_{i}\right)
$$

le sous-espace engendré par X étant $\omega_{\varphi}(\mathrm{X})$.

E est élément universel et $\omega_{\varphi}(\varnothing)$ élément nul, mais les atomes ou points n'existent pas toujours, les sous-espaces de la forme $\omega_{\varphi}(x)$ pouvant etre inclus les uns dans les autres suivant des chaines descendantes infinies.

Soit $\underset{\mathrm{m}}{S}<$ la catégorie dont les objets sont les couples $(E, \mathcal{J})$ où $\mathcal{J}_{\subset} \$(E)$ vérifie les axiomes $\left(S_{1}\right)$ et $\left(S_{2}\right)$, les morphismes de $\left(E, \varphi^{\prime}\right)$ dans $\left(E^{\prime}, \mathcal{Y}^{\prime}\right)$ étant les applications $f$ de $E$ dans $E^{\prime}$ telles que :

$$
\forall A^{\prime} \in \varphi^{\prime} \quad, \quad f^{-1}\left(A^{\prime}\right) \in \mathcal{P}
$$

Théorème 6 (Schmidt - P. Hall)

$F:(E, \varphi) \longrightarrow(E, T(E, \varphi)) \quad$ définit un foncteur ensembliste de $\underset{m}{\Phi} \mathbf{f}^{<}$sur $\underset{m}{S^{<}}$, et $\quad \mathrm{G}:(E, \varphi) \longrightarrow\left(E, \varphi_{\varphi}\right)$ où :

$$
\forall x \in \beta(E), \varphi_{\mathcal{S}}(\mathrm{X})=\bigcap_{\mathrm{A}} \quad[\mathrm{A} \in \mathcal{S} \text { et } \mathrm{X} \subset \mathrm{A}]
$$

définit un foncteur ensembliste de $\underset{\mathrm{m}}{S}<$ sur $\underset{\mathrm{min}}{F^{\alpha}}$. Soit $F_{0}$ la restriction de $F$ a $\underset{m}{F^{<}}, F_{0}$ et $G$ sont des isomorphismes inverses l'un de l'autre entre $\underset{m}{F_{m}}$ et $\underset{\mathrm{m}}{S^{<}}$

(*) Ce résultat a été démontré sous une forme un peu plus faible (équivalence de structures) par J. Schmidt [22] et P. Hall (non publié) (voir [Q], chap. II, 5., p. 81) . 


\section{Démonstration :}

Il est aisé de démontrer que $\varphi_{\mathcal{Y}}$ vérifie $\left(G_{2}\right)$ et $\left(G_{6}\right)$. L'on démontre que, pour tout $X \in E, \varphi_{\mathcal{Y}}(X)=U_{\varphi_{\mathcal{Y}}}(\mathrm{Y}) \quad[\mathrm{Y} \in \mathfrak{T}(\mathrm{X})]$, par récurrence transfinie sur Card X. C'est trivial si $X$ est fini. Supposant ceci vrai si Card $X<a$, soit $z \subset E$ tel que $C a r d Z=a$. Alors il existe au moins une famille $\left(z_{i}\right)_{i \in I}$ striotement croissante de parties de $z$ telles que :

$$
\forall i \in I \quad \text { Card } z_{i}<a \quad \text { et } \quad z=\underset{i}{\cup_{i}} z_{i}
$$

Alors :

$$
\varphi_{\mathscr{Y}}(z)=\bigcup_{i} \varphi_{\mathcal{Y}}\left(z_{i}\right)=\bigcup_{i} \bigcup_{Y \in \mathcal{F}^{\mathcal{F}}\left(z_{i}\right)} \varphi_{\mathcal{Y}}(Y)=\bigcup_{Y \in \mathcal{I}^{P}(X)} \varphi_{\mathcal{J}}(Y)
$$

L'on vérifie aisément que $F$ et $G$ sont des foncteurs ensemblistes en remarquant que :

$$
\left(f \circ \varphi \subset \varphi^{\prime} \circ f\right) \Longleftrightarrow\left(\varphi \circ f^{-1} \subset f^{-1} \circ \varphi^{\prime}\right)
$$

Mais $F$ n'est plus un foncteur ensembliste de $\underset{m}{\Phi}$ dans $\underset{m}{S}$, surcatégorie de $\underset{m}{S}$ obtenue en transportant les morphismes de $\underset{m}{F}$ (F est isomorphe au foncteur de fermeture).

$$
\begin{aligned}
& \text { Soit } \varphi \text { une } f \text { g sur } E \text {. L'on pose : } \\
& \mathscr{L}_{\varphi}=\{X / X \subset E \text { et } \forall x \in X, x \notin \varphi(X-x)\}
\end{aligned}
$$

et

$\mathscr{L}_{\varphi}^{<}=\{X / X \subset E$ et il existe un ordre total sur $X$ tel que :

$$
\forall x \in X \quad, \quad x \notin \varphi(] \leftarrow, x[)\}
$$

$\mathscr{L}_{\varphi}$ et $\mathscr{L}_{\varphi}^{<}$sont de caractère fini et $\mathscr{L}_{\varphi} \subset \mathscr{L}_{\varphi}^{<}$

$$
\mathbf{r}=\mathbf{r}_{\mathscr{L}}^{<} \text {est le rang associé à } \varphi
$$

et $\quad r S=r_{\mathscr{L}_{\varphi}}$ est le rang symétrique associé $\varphi$. 
Soit $X \subset E . \quad \varphi_{X}: Y \subset E \longrightarrow \varphi_{X}(Y)=\varphi(X \cup Y)$ est une $f g f \cdot$ Si $\varphi$ est une fermeture, $\varphi_{X}$ en est une aussi. L'on écrit $r_{X}$ et $r S_{X}$ les rangs associés à $\varphi_{\mathbf{X}} \cdot$

$(E, \varphi)$ est dit semi-régulière si, pour toute partie $X$ de $E$, pour tout sous $\varphi_{X}$-espace A de $E$, pour toute partie $\mathscr{L}_{\varphi_{X}}^{<}$-libre maximale $Y$ incluse dans $A$, on a Card $Y=r_{X}(A)$

L'on appelle $\varphi$-base de A toute partie $Y \mathscr{L}_{\varphi}^{<}$-libre maximale de A. Alors $\varphi(Y)=A$. Mais en général il existe des parties $\mathscr{L}_{\varphi}^{<}$-libre $Z$ de $A$ telles que $\varphi(z)=$ A. $z$ sera alors appellé une base faible de A.

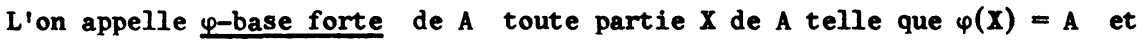
$\forall x \in X, \varphi(X-x) \subsetneq A$ (alors $\left.X \in \mathscr{L}_{\varphi}\right)$. Un sous-espace $A n^{\prime}$ admet pas en général de base forte. L'on a cependant la :

Proposition $3\left(^{*}\right)$ Soit $\varphi$ une fermeture sur E et A un sous-espace. Si A admet une base forte finie, toutes les bases fortes de A sont finies (mais r S (A) peut être dénombrable). Si A admet une base forte infinie B, toutes les bases fortes de A sont infinies et ont pour cardinal rS $(A)=$ Card B.

\section{démonstration :}

Soient $L$ et $M$ deux bases fortes de A. Si $L$ est infinie, on a Card $M \geqslant$ Card $L$. En effet, pour tout $l \in L$, il existe $M_{\ell} \in \mathcal{G}(M)$ tel que $l \in \varphi(M)$. Or ;

$$
\forall l \in L \quad L_{0}=\left\{h / h \in L \quad \text { et } M_{h}=M_{l}\right\} \quad \text { est fini. }
$$

(*) Enoncé analogue à celui de $[Q]$, chap II , 5., p. 82 
En effet pour tout $m \in M_{\ell}$, il existe $L_{m} \Leftarrow f(L)$ tel que $m \in \varphi\left(L_{m}\right)$. Done $L_{M_{\ell}}$ est fini et $\varphi\left(L_{M_{\ell}}\right) \supset M_{\ell}$, d'où $\varphi\left(L_{M_{\ell}}\right)>\varphi\left(L_{0}\right)$,

$$
\varphi\left(\mathrm{L}_{M_{\ell}} \cup \mathrm{L}_{0}\right)=\varphi\left(\mathrm{L}_{0}\right) \quad \text { et } \quad \mathrm{L}_{0} \subset \mathrm{L}_{M_{l}}
$$

Donc $\mathcal{f}(M)$ est infini et :

Card $M=\operatorname{Card} \mathcal{F}(M) \geq U_{0} \times \operatorname{Card} L=\operatorname{Card} L$

Comme Card $M$ est infini, on a de même Card $L \geqq$ Card $M$ d'où égalité.

Remarque : En général si A est un sous-espace de $(E, \varphi)$, rS $(A)<r(A)$, même quand $r(A)$ est infini.

$(E, \varphi)$ est un $\varphi$-espace si et seulement si $T(E, \varphi)$ est un treillis géométrique (voir [E], IIIe partie).

Inversement à tout treillis géométrique $T$ correspond le $\varphi$-espace $P(T)$ des points de $T$, où pour tout $X \subset P(T)$,

$$
\varphi(X) \text { est l'ensemble des points } \quad x \leqslant \vee y \quad[y \in X]
$$

(L'on verra au chapitre II, 2.4.1 les foncteurs canoniques qui lient ces structures).

\subsection{4.- Autres systèmes d'axiomes équivalents pour les $\varphi$-espaces.}

Voici des axiomes concernant les familles libres ou liées :

$1^{\circ}$ ) Axiomes de Steinitz-Van der Waerden. (voir $[\mathrm{I}]$ et $(\mathrm{J}]$ ) sont les axiomes des $\varphi$-espaces.

$2^{\circ}$ ) Axiomes de Haupt Otto, Nöbeling et Pauc. (voir [3]). Soit F un ensemble et $\mathcal{F}$ l'ensemble des familles finies $\left(x_{1}, \ldots, x_{n}\right)$ d'éléments de $E$. On considère

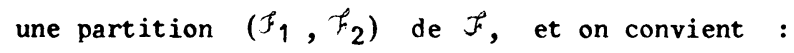


$\left(x_{1}, \ldots, x_{n}\right) \in \mathcal{F}_{1} \quad$ s'écrit $A\left[x_{1}, \ldots, x_{n}\right] \quad$ (parties liées) (abhängig)

$\left(x_{1}, \ldots, x_{n}\right) \in \Im_{2} \quad s^{\prime}$ 'écrit $U\left[x_{1}, \ldots, x_{n}\right] \quad$ (parties libres) (unabhängig)

avec les axiomes :

(K) si $x_{1}=x_{2}$, on a $A\left[x_{1}, x_{2}\right]$

(I) si $A\left[x_{1}, \ldots, x_{n}\right]$, alors pour tout $y \in E$, on a $A\left[x_{1}, \ldots, x_{n}, y\right]$

(A) si $U\left[x_{1}, \ldots, x_{p}\right], \quad A\left[x_{1}, \ldots, x_{p}, y\right]$ et $A\left[x_{1}, \ldots, x_{p}, z\right]$

on a $A\left[x_{2}, \ldots, x_{p}, y, z\right]$.

$3^{\circ}$ ) Axiomes de Rado (voir [9])

Soient $\mathrm{F}, \mathcal{F}$ (comme en 2$)$ et $\underline{I}$ une relation d'indépendance :

I application de $\mathcal{F}$ dans $\{0,1\}$ telle que :

1) - I est décroissante : $I\left(x_{1}, \ldots, x_{n}\right) \geq I\left(x_{1}, \ldots, x_{n}, x_{n+1}\right)$

2) - I est commutative : $I\left(x_{1}, \ldots, x_{n}\right)=I\left(x_{\sigma(1)}, \ldots, x_{\sigma(n)}\right)$

$\forall \sigma \in \mathbf{n}$ !

3) - I est non reflexive : $I(x, x)=0$

4) - I est distributive; i.e.

$I\left(x_{1}, \ldots, x_{n}\right) I\left(y_{1}, \ldots, y_{n+1}\right)=\sum_{k=1}^{n+1} I\left(x_{1}, \ldots, x_{m}, y_{k}\right)$

\subsection{5.- Principales propriétés des $\varphi$-espaces}

(a) Toute partie libre maximale $\mathrm{Y}$ de $\mathrm{A}$ est appellée base de $\mathrm{A}$. Toute base de A est une base de $\varphi(A)$

(b) (Théorème d'ŕchange). Si $\varphi(Y)=A$ et si X est une partie libre de $A$, il existe $Y^{\prime} \subset Y$ telle que $Y^{\prime} \cap X=\varnothing$ et $Y^{\prime} U X$ soit une base de $A$. 
(c) Soit B une base de A. Toute base de A a même cardinal que B. Card $B=\operatorname{dim}_{\varphi} A=r_{\varphi} A$ est appellé la dimension de $A$. En outre, si $Y$-engendre $A$, Card $Y \geq \operatorname{dim}_{\varphi} A$; et si $Y$ est une partie libre de $A, \operatorname{Card} Y \leqslant \operatorname{dim}_{\varphi} A$; et quand $\operatorname{dim}_{\varphi}$ A est fini (resp. infini), l'égalité dans l'un ou l'autre de ces cas entraîne (resp. n'entraîne pas en général) que A soit une $\varphi$-base de $A$.

(d) Soit $L$ une partie libre et $x \in E . L+x$ contient un circuit et un seul :

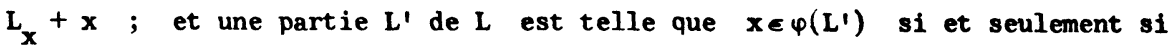
$\mathbf{x} \in \varphi(\mathrm{L})$ et $\mathrm{L}^{\prime} \supset \mathrm{L}_{\mathbf{x}}$.

(e) Si $\left(L_{i}\right)_{i \in I}$ est une famille de parties d'une partie libre $L$,

$$
\varphi\left(\cap L_{i}\right)=\cap \varphi\left(L_{i}\right)
$$

\section{5.- Applications et exemples}

\subsection{1.- A-modules et groupes}

Soit $M$ un A-module à gauche unitaire et, pour tout $X \subset M, \varphi_{0}(X)$ le sous-A-module engendré par $X$

$$
\mathscr{L}_{0}=\mathscr{L}_{\varphi_{0}} \quad \text { est } 1 \text { 'ensemble des parties libres usuelles, et } \varphi_{0} R(X) \text { est la }
$$
cloture de X

Soit $\mathscr{L}_{<} l^{\prime}$ 'ensemble des parties $X$ de $M$ telles qu'il existe un ordre total sur $\mathrm{X}$ pour lequel :

$$
\begin{aligned}
& \forall x \in X \quad, \quad x \notin \varphi_{0} \quad\left(x_{] \leftarrow,} \quad x[)\right. \\
& \left(E, \mathscr{L}_{c}\right) \text { est un } \mathscr{L}_{\text {-espace semi-régulier. }}
\end{aligned}
$$

En effet $\mathscr{L}_{<}$est de caractère fini (raisonner par limite projective de manière analogue au théorème 5 de 1.3.3.). Le fait que $\left(E, \mathscr{L}_{<}\right)$soit semi-régulier résulte du théorème de Jordan-Holder (voir par exemple Bourbaki $[\mathrm{C}, \mathrm{II}, 1], \S 6$, n० 14, p. 85) 


$$
\forall x \in \beta(M), \quad r_{<}(x)=\text { long }\left(\varphi_{0}(x)\right)
$$

est définie même quand la longueur est infinie (voir Bourbaki $[\mathrm{C}, \mathrm{II}, 2], \S 1$, $\mathrm{n}^{\circ} 10$, p. 30 )

$M$ est un espace vectoriel si et seulement si $\mathscr{L}_{0}=\mathscr{L}_{<}$, et alors $\left(M, \mathscr{L}_{0}\right)$ est un $\varphi$-espace.

L'on a des résultats analogues pour les groupes à opérateurs.

1.5.2.- Fnsembles ordonnés et treillis.

Soit $(E, \leq)$ un ensemble ordonné et $\mathscr{L}$ l'ensemble des parties $X$ de $E$ telles que $(\mathrm{x}, \leq$ induit $)$ soit totalement ordonné. $\mathcal{L}$ est de caractère fini et $\mathscr{C}$ est l'ensemble des $\{x, y\}$ tels que $x$ et $y$ ne soient pas comparables.

$(\mathrm{E}, \mathcal{L})$ est un $\underline{\varphi \text {-espace }}$ si et seulement si la relation "x et $y$ ne sont pas comparables" sur $E$ est transitive. $(E, \leq)$ est alors dit régulier.

\subsection{3.- Corps}

Soit $K$ un corps, $k$ un sous-corps de $K$ et $\varphi(X)$ la clôture algébrique de $\underline{k(X) \text { dans } K}$ (voir $[L]$, vol $I$, chapitre II, $\S 12) .(K, \varphi)$ est régulière et l'on retrouve ici les propriétés classiques des parties algébriquement libres et du degré de transcendance. 
Soit A un anneau unitaire et pour tout $X \subset E, \varphi_{1}(x)$ l'idéal bilatère engendré par $x$. Soit $\mathscr{L}_{<}$défini comme en 1.5.1. pour $\varphi_{1} \cdot\left(E, \mathscr{L}_{<}\right)$est semi-régulier (voir A-module).

Soit $k$ un corps de caractéristique $p>0$ et pour tout $x<E, \varphi(x)=k^{P}(X)$. $(k, \varphi)$ est un $\varphi$-espace (voir $[L]$, vol. I, chapitre II, $\S 17$ ). Les bases sont alors appellées les p-bases.

\subsection{4.- Les "Géométries".}

Toute géométrie $G$ est telle que l'ensemble de ses points soit canoniquement un $\varphi$-espace. En particulier :

a. Tout espace affine sur un corps $K$

b. Tout espace projectif sur un corps $K$ (voir [2], §9, pp. 189-205 ou [E], III, chap. IV, pp. 354 à 373)

c. Toute géométrie plane affine, de translation ou non, desarguesienne ou non, pascalienne ou non (voir[E], III, chap V, pp. 318-353)

d. Toute géométrie plane projective (voir [F], chap. 20, pp. 346-420). 
Chap. II LES STRUCTURES DE $\mathscr{L}$-ESPACE ET DE $\varphi$-ESPACE

\section{1.- Généralités. Comparaisons de Structures}

\subsubsection{Rappels sur les catégories de base.}

L'on entend par $\mathscr{L}$-espace la donnée d'un ensemble muni de l'une des structures équivalentes de la planche $I$, et l'on désigne par L la catégorie dont les objets sont les $\mathscr{L}$-espaces et dont les morphismes de $(\mathrm{F}, \mathscr{L})$ dans $\left(E^{\prime}, \varphi^{\prime}\right)$ sont définis par l'une des propriétés équivalentes :

$$
\left(h_{1}\right),\left(h_{2}\right),\left(h_{3}\right) \text { ou }\left(h_{4}\right)
$$

( L'on identifie ainsi les catégories $L_{m}, \underset{m}{R}$ et $\Phi_{m}^{g}$ ).

Pour differencier plusieurs structures on pourra employer des indices : ainsi $\mathscr{L}_{2}^{\prime}$ est $l^{\prime}$ ensemble des parties $\varphi_{2}^{\prime}$-libres de $\left(E, r_{2}^{\prime}\right)$.

Du point de vue des fonctions génératrices, il est naturel de définir la catégorie $\underset{\mathrm{m}}{\mathrm{L}}=\left(\operatorname{resp} . \underset{\mathrm{m}}{L^{<}}\right)$ayant pour objets les $\mathscr{L}$-espaces, les morphismes de $(E, \varphi)$ dans $\left(E^{\prime}, \varphi^{\prime}\right)$ étant les applications $f$ de $(E, \varphi)$ dans $\left(E^{\prime}, \varphi^{\prime}\right)$ telles que :

$$
\begin{array}{ll}
\left(h_{1}^{=}\right) & \text {f० } \varphi=\varphi^{\prime} \circ f \\
\left(\operatorname{resp} .\left(h_{1}^{<}\right)\right. & \text {f } \left.\circ \varphi \subset \psi^{\prime} \circ f\right)
\end{array}
$$

On a

$$
\underset{m m}{\mathbf{L}} \subset \underset{m}{\mathbf{L}}<\underset{m}{\mathbf{L}}
$$

La première inclusion est évidente. Pour la seconde : si $f$ est un $L_{\text {mm-morphisme }}$ de $(E, \mathscr{L})$ dans $\left(E^{\prime}, \mathscr{L}^{\prime}\right)$, soit $\left(x_{i}\right)_{i \in I}$ une famille d'élćments de $E$ telle que $\left(f\left(x_{i}\right)\right)$ soit $\mathscr{L}^{\prime}$-libre. Alors les $x_{i}$ sont distincts deux à deux et, considérant un bon ordre sur I : 


$$
\begin{array}{ll}
\forall i \in I & f\left(x_{i}\right) \notin \varphi^{\prime} \quad\left(\left\{f\left(x_{j}\right) / j \in I \text { et } j<i\right\}\right) \\
\text { donc } & f\left(x_{i}\right) \notin f \circ \varphi\left(\left\{x_{j} / j \in I \text { et } j<i\right\}\right) \\
\text { et à fortiori } & x_{i} \notin \varphi\left(\left\{x_{j} / j \in I \text { et } j<i\right\}\right) \\
\text { donc }\left(x_{i}\right) \text { est } \mathcal{L} \text {-libre. }
\end{array}
$$

De même l'on entend par $\varphi$-espace la donnée d'un ensemble muni de l'une des structures équivalentes de la planche II, et l'on désigne par $\oint_{\text {m }}$ (resp. $\Phi_{m}^{=}$; resp. $\underset{m}{\Phi^{<}}$) la sous-catégorie pleine de $\underset{m}{L}$ (resp. $\underset{m}{L}=$; resp. $\mathrm{L}_{m}^{<}$) dont les objets sont les $\varphi$-espaces. On a :

$$
\Phi_{m}=c \Phi_{m}^{<}<\underset{m}{\Phi}
$$

Propriétés caractéristiques des L-morphismes (resp. des $\Phi$-morphismes)

$$
\text { Une application } f \text { de }(E, \mathscr{L}) \text { dans }\left(E^{\prime}, \mathscr{L}^{\prime}\right) \text { est un } \underline{L}-(\text { resp. } \Phi) \text {-mor- }
$$
phisme si et seulement si elle vérifie l'une des propriétés équivalentes suivantes $\left(h_{1}\right)$ toute famille $\left(x_{i}\right)$ telle que $\left(f\left(x_{i}\right)\right)$ soit $\mathscr{L}^{\prime}$-libre, est $\mathscr{L}$-libre

$$
\left(h_{2}\right) \quad \forall x \in \mathcal{F}(E), \quad r^{\prime}(f(x)) \leq r(x)
$$

$\left(h_{3}\right)$ Si $\left(x_{i}\right)$ est $\mathscr{L}$-liée, $\quad\left(f\left(x_{i}\right)\right)$ est L''-liée

$\left(h_{4}\right) \quad \forall c \in C \quad, \quad(f(x))_{x \in C} \quad$ est $\mathscr{L}^{\prime}$-liée

$\left(h_{5}\right) \quad \forall x \in f(E)$ tel que $f / x$ injective et $f(x)$ base de $f(E), \quad x \in \mathcal{Z}$

(h6) $\forall x \in G$ tel que $\left.f\right|_{x}$ injective, $f(x)$ contient au moins un circuit.

$\left(h_{7}\right) \quad \forall x \in f(E)$ tel que : $\exists x \in X, \quad x \in \varphi(X-x) ; \exists y \in X, \quad f(y) \in \varphi^{\prime} \quad(f(X-y))$ 
Propriétés caractéristiques des $L^{<}$-morphismes (resp. des $\Phi$-morphismes).

La notion suivante est souvent utile : Une famille $\left(x_{i}\right)_{i \in I}$ d'éléments d'un $\mathscr{L}$-espace est appellée un pseudo-circuit si :

$\forall i \in I \quad x_{i} \in \varphi \quad\left(\left\{x_{j} \mid j \in I-i\right\}\right)$

En particulier une partie $X$ de $E$ est un pseudo-circuit si $(x)_{x \in X}$ en est un. Il est clair qu'alors, pour $\mathrm{X} \in \mathrm{E}$, les propriétés suivantes sont équivalentes :

1) $X$ est un pseudo-circuit

2) $\forall x \in X, \quad x \in \varphi(X-x)$

3) $X$ est réunion de circuits

L'on désignera par $P \mathscr{C}$ ou $P(\mathscr{C})$ l'ensemble des $\mathscr{C}$-pseudo-circuits.

Une application $f$ de $(E, \mathscr{L})$ dans $\left(E^{\prime}, \mathscr{L}^{\prime}\right)$ est un $\underline{L}^{<}-\left(\right.$resp. $\left.\Phi^{<}-\right)$morphisme si et seulement si elle vérifie l'une des propriétés équivalentes suivantes:

$\left(h_{1}^{<}\right)$ $f \circ \varphi<\varphi^{\prime} \circ f$

$\left(h_{1}^{<} f\right)$

$\forall x \in \mathcal{I}^{(E)}$,

$f \circ \varphi(X) \subset \varphi^{\prime} \circ f(X)$

$\left(h_{2}^{<}\right)$

$\varphi \circ f^{-1} \subset f^{-1} \circ \varphi^{\prime}$

$\left(h_{3}^{<}\right)$

$\forall x \in \zeta \quad, \quad(f(x))_{x \in X}$ est un pseudo-circutt de $E^{\prime}$

$\left(h_{4}^{\leqslant}\right)$

$\forall x \in \beta(E)$ tel que : $\forall x \in X, \quad x \in \varphi(x-x)$, on a : $\forall y \in X$, $y \in \varphi^{\prime}(f(X-y)) \quad$ (comparer avec $\left.\left(h_{7}\right)\right)$

et (pour les $\varphi$-espaces seulement) :

$\left(h_{5}^{<}\right) \quad \forall A^{\prime} \in \mathcal{S}^{\prime} \quad, f^{-1}\left(A^{\prime}\right) \in \mathcal{J}$ 
Propriétés caractéristiques des $\Phi^{-}$-morphismes

$$
\text { Une application } f \text { de }\left(E, \mathscr{L}^{\prime}\right) \text { dans }\left(E^{\prime}, \mathscr{L}^{\prime}\right) \text { est un } \underline{\Phi}^{=} \text {-morphisme }
$$

si et seulement si elle vérifie l'une des propriétés équivalentes suivantes :

$\left(h_{1}^{=}\right) \quad$ f० $\varphi=\varphi^{\prime} \circ f$

$\left(\mathrm{h}_{2}\right) \quad \forall \mathrm{A}^{\prime} \in \mathcal{Y}^{\prime}, \quad \mathrm{f}^{-1}\left(\mathrm{~A}^{\prime}\right) \in \mathcal{S} \quad$ et $\forall \mathrm{A} \in \mathcal{S}, \mathrm{f}(\mathrm{A}) \in \mathcal{S}^{\prime}$.

L'on peut aussi les caractériser à l'aide des circuits. Les structures image réciproque et image directe permettront de préciser ces morphismes.

\section{$\underline{\text { 2.1.2.- Comparaisons de structures }}$}

L'ensemble $\underset{\operatorname{mim}}{L(E)}$ des structures de $\mathscr{L}$-espace sur $E$ est ordonné (voir Bourbaki [C, I, 4], $\S 2, \mathrm{n}^{\circ} 2$, p. 25) par l'une des propriétés équivalentes ci-dessous :

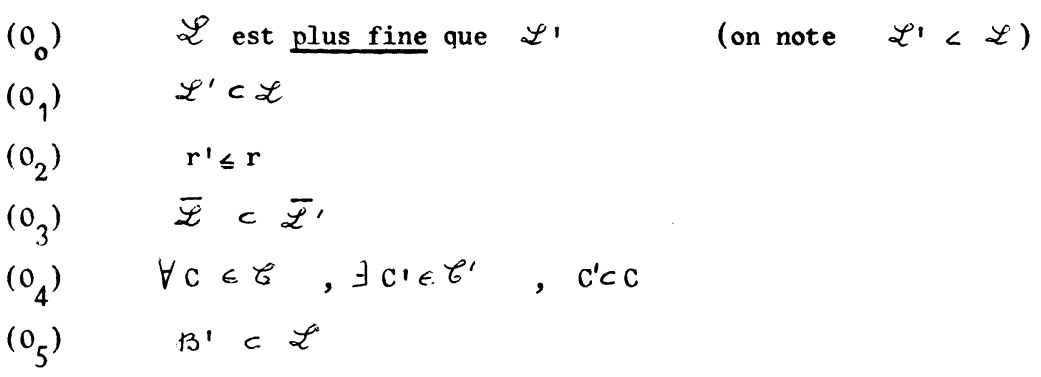

De même l'ensemble ordonné $L_{m}^{<}(E)$ est ordonné par l'une des propriétés équivalentes ci-dessous : 
$\left(0 \mathrm{~F}_{\mathrm{o}}\right) \quad \mathscr{L}$ est fortement plus fine que $\mathscr{L}^{\prime} \quad$ (on note $\mathscr{L}^{\prime} \ll \mathscr{L}$ )

$\left(0 F_{1}\right) \quad \varphi \subset \varphi^{\prime}$

$\left(0 \mathrm{~F}_{2}\right) \quad \forall \mathrm{x} \subset \ell, \mathrm{X}$ est réunion de $\mathscr{C}^{\prime}$-circuits (i.e. $\mathscr{C} \subset \mathrm{P} \mathscr{C}^{\prime}$ )

et, pour les $\varphi$-espaces seulement :

$(0 \mathrm{~F} \Phi) \quad \varphi^{\prime} \subset \mathcal{Y}$

L'ordre de $\underset{m}{L}=(E)$ se réduit à l'égalité .

Si $\mathscr{L}$ est fortement plus fine que $\mathscr{L}^{\prime}, \mathscr{L}$ est plus fine que $\mathscr{L}^{\prime}$.

Propriétés de l'ensemble ordonné I(E).

La structure la plus fine est caractérisée par :

$\mathscr{L}=\beta(E) ; \mathscr{C}=\varnothing ; \forall \mathbf{X} \in \beta(E), \mathbf{r}(\mathrm{X})=\operatorname{Card} \mathbf{X}$ et $\varphi(\mathrm{X})=\mathbf{X} ; \beta=\{E\}$.

c'est la structure de $\varphi$-espace discret sur E.

La structure la moins fine est caractérisée par :

$\mathscr{L}=\{\varnothing\} ; \mathscr{C}=\mathcal{F}_{1}(\mathrm{E}) ; \forall \mathbf{X} \in \beta(E), \quad \mathbf{r}(\mathbf{X})=0 \quad$ et $\varphi(X)=\mathbf{E} ; \beta=\{\varnothing\}$

c'est la structure de $\varphi$-espace grossier sur $\mathrm{E}$.

L'on démontre alors sans difficultés le :

Théorème 1.- $L(E)$ est un treillis complet. Plus précisément :

(1) $\wedge \mathscr{L}_{i}=\cap \mathscr{L}_{i} \quad ; \quad$ Si I est fini, $\underset{i \in I}{\vee} \mathscr{L}_{i}=\bigcup_{i \in I} \mathscr{L}_{i}$

et dans le cas général :

$$
\checkmark \mathscr{L}_{i}=\left\{x \mid x \subset E \text { et } \forall y \in \mathscr{F}(x), \quad \exists i \in I, \quad Y \in \mathscr{L}_{i}\right\}
$$

(2)

$$
\forall x \in \mathcal{F}(E) \quad, \quad\left(\underset{i}{V} r_{i}\right)(x)=\operatorname{Max}_{i} r_{i}(x)
$$




$$
\text { et soit } \quad \rho(x)=\underset{i}{\operatorname{Min}} \mathbf{r}_{\mathbf{i}}(\mathrm{x}),
$$

$\rho$ est un rang faible, mais non un rang en général. Donc :

$$
\hat{\mathbf{i}} \quad \mathbf{r}_{\mathbf{i}}=\hat{p}=\mathbf{r}_{\mathcal{L}_{p}} \quad \text { (voir corollaire } 3 \text { du théorème } 1 \text { de 1.1.) }
$$

(3) (*) $\wedge \mathscr{C}_{i}=\operatorname{MIN}\left(\cup_{i} \mathscr{C}_{i}\right) \quad$ et $\vee \mathscr{C}_{i}=\operatorname{MIN}\left(\cap_{i} \bar{L}_{i}\right) \cap$ fr (E)

Ce treillis est distributif, mais non complémenté.

Propriétés des ensembles ordonnés $h^{<}(E)$ et $\Phi^{<}(E)$

Pour ces ensembles ordonnés, la structure la moins fortement fine est la structure grossière, et la structure la plus fortement fine est la structure discrète.

L'on démontre aisément la :

Proposition 1.- $\underline{L}^{<}(E)$ et $\Phi^{<}(E)$ sont des ensembles ordonnés inductifs pour leur ordre et pour leur ordre inverse. Plus précisément, $\left(\mathscr{C}_{i}\right)$ étant une famille

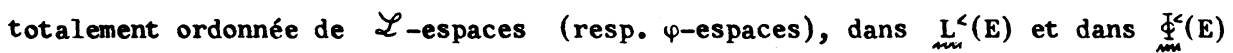
on a :

$$
\begin{array}{rlrl}
\vee & \mathscr{C}_{i}=\vee \vee \mathscr{C}_{i} & \wedge \mathscr{C}_{i}=\wedge \mathscr{C}_{i} \\
p\left(\vee \mathscr{C}_{i}\right) & =\cap p \quad \mathscr{C}_{i} & & \text { et } p\left(\wedge \mathscr{C}_{i}\right)=\vee p \mathscr{C}_{i} \\
\vee \varphi_{i} & =\cup \varphi_{i} &
\end{array}
$$

(*) Si X est une partie d'un ensemble ordonné, I'on désigne par MIN (X) (resp. MAX (X)) l'ensemble des éléments minimaux (resp. maximaux) de X. 
où

$$
\forall \mathrm{X} \in \mathrm{E}, \quad \boldsymbol{\theta}(\mathrm{X})=\mathrm{U}\left(\cap \varphi_{\mathrm{i}}(\mathrm{Y})\right) \quad[\mathrm{Y} \in \mathcal{F}(\mathrm{X})]
$$

(en général $\cap \varphi_{i}$ ne vérifie pas $\left(G_{1}\right)$ ).

Ces ensembles ordonnés ne sont plus des treillis, les bornes supérieures ou inférieures n'existant plus en général, même pour deux éléments.

2.1.3. - Exemple fondamental : $\mathscr{L}_{\mathrm{A}}$ ou $\mathscr{L}[\mathrm{A}], \mathrm{r}_{\mathrm{A}} \cdots$

\section{Théorème et définition 2.-}

Soit $\mathscr{C}$ une structure de $\mathscr{L}$-espace sur $\mathrm{E}$ et ACE. Alors :

$\left(\mathrm{a}_{1}\right) \quad \mathscr{C}_{\mathrm{A}}=\mathscr{C}[\mathrm{A}]=\mathcal{F}_{1}(\mathrm{~A}) \cup \operatorname{MIN} \quad(\{\mathrm{C}-\mathrm{A} \mid \mathrm{C} \in \mathscr{C}\}-\{\varnothing\})$

définit sur $E$ une structure de $\mathscr{L}$-espace moins fine que $\mathscr{C}$ appellée la translatée de $C_{\text {par A }}$ et ayant les propriétés suivantes :

$$
\begin{aligned}
& \left(\mathrm{a}_{2}\right) \quad \mathscr{L}_{\mathrm{A}}=\mathscr{L}_{[\mathrm{A}]}=\{\mathrm{x} \mid \mathrm{x} \subset \mathrm{E}, \mathrm{x} \cap \mathrm{A}=\varnothing \text { et } \forall \mathrm{C} \in \mathcal{C}, \quad \mathrm{c} \notin \mathrm{x} \cup \mathrm{A} \text { ou } \mathrm{c} \cap \mathrm{X}=\varnothing\} \\
& \left(\mathrm{a}_{3}\right) \quad \forall \mathrm{X} \in \beta(\mathrm{E}), \quad \varphi(\mathrm{A}) \cup \mathrm{X} \subset \varphi_{\mathrm{A}}(\mathrm{X}) \quad \subset \quad \varphi(\mathrm{A} \cup \mathrm{X}) \\
& \left(a_{4}\right) \quad\left(\forall x \in \beta(E), \quad \varphi_{A}(x)=\varphi(A \cup X)\right) \Longleftrightarrow\left(\zeta_{[A]} \ll \zeta\right) \\
& \left(a_{5}\right) \quad \forall x \in \mathcal{F}(E), \quad r_{A}(X) \leq \operatorname{Min}(r(X \cup B)-r(B)) \quad[B \in \mathcal{F}(A)]
\end{aligned}
$$

si $\mathscr{C}$ est une structure de $\varphi$-espace, $\mathscr{C}[\mathrm{A}]$ est aussi un $\varphi$-espace et alors $\mathscr{C}[\mathrm{A}]$ est fortement moins fine que $\mathscr{C}$ et, outre $\left(\mathrm{a}_{4}\right)$, on a l'égalité dans $\left(a_{5}\right)$.

\section{Démonstration}

$$
\left(a_{1}\right) \text { définit } \zeta_{A} \text { et } \mathscr{C}_{A}<\zeta \text { est trivial. On utilisera } \mathscr{C}[\mathrm{A}] \text { pour }
$$
éviter les indices inférieurs. 
L'on vérifie $\left(\mathrm{a}_{2}\right)$ en démontrant d'abord que :

$$
\overline{\mathscr{P}}_{\mathrm{A}}=\{\mathrm{X} \mid \mathrm{X} \subset \mathrm{E}, \quad \mathrm{X} \cap \mathrm{A} \neq \varnothing \text { ou } \exists \mathrm{c} \in \mathscr{C} \text { tel que } \mathrm{C} \in \mathrm{X} \cup \mathrm{A} \text { et } \mathrm{C} \cap \mathrm{X} \neq \emptyset\}
$$

Il est en général difficile de calculer $\varphi_{A}$ et $r_{A}$ autrement que par les formules de la planche $I$. Cependant $\left(a_{3}\right)$ et $\left(a_{5}\right)$ donnent des bornes remarquables et $\left(a_{4}\right)$ donne $\varphi_{A}$ quand $\mathscr{C}_{A}$ est fortement plus fine que $\mathscr{C}$.

$$
\begin{array}{ll}
\text { D'après } & \varphi_{\mathscr{C}}, \varphi_{A}(\varnothing)=\varphi(A) \text {. D'où : } \\
\forall X \in \beta(E), & \varphi_{A}(X) \supset \varphi(A) \cup X .
\end{array}
$$

Soit maint enant :

$$
\mathscr{C}_{1}=\mathrm{MIN} \quad\left(\{\mathrm{C}-\mathrm{A} \mid \mathrm{c} \in \zeta\}-\{\phi) \quad \text { et } \quad \zeta_{0}=\left\{\mathrm{c} \mid \mathrm{c} \in C^{-} \text {et } \mathrm{C}-\mathrm{A} \in \mathscr{C}_{1}\right\}\right.
$$

on a :

$$
\begin{aligned}
& \forall X \in \beta(E), \quad \varphi_{A}(X)=A \cup X \cup\left\{x \mid X \in E \text { et } \exists Y \in \mathcal{F}_{(A \cup X),} Y+x \in C_{0}\right\} \\
& \text { or } \mathscr{C}_{0} \subset \mathcal{C} \text {, donc }: \varphi_{A}(\mathrm{X}) \subset \varphi(\mathrm{A} \cup \mathrm{X})
\end{aligned}
$$

si $\mathscr{C}_{\mathrm{A}} \ll \mathscr{C}, \quad \mathrm{Y} x \in \varphi(\mathrm{A} \cup \mathrm{X})-\mathrm{A} \cup \mathrm{X}, \exists \mathrm{Y} \in \mathcal{F}(\mathrm{A} \cup \mathrm{X}), \quad \not+\mathrm{x} \in \mathscr{C}$. Comme $\mathrm{Y}+\mathrm{X}$ est réunion de circuits de $\mathscr{C}_{\mathrm{A}}$, il existe $Z \in \mathcal{F}(\mathrm{Y})$ tel que $z+x \in \mathscr{C}_{A}$. Donc $\mathrm{X} \in \varphi_{\mathrm{A}}(\mathrm{X})$ et $\quad \varphi_{\mathrm{A}}(\mathrm{X}) \supset \varphi(\mathrm{A} \cup \mathrm{X})$.

si $\mathscr{C}_{\mathrm{A}}</<\mathscr{C}, \exists \mathrm{c} \in \mathscr{C}, \exists \mathrm{x} \in \mathrm{C}$ tels que $\mathrm{x}$ n'appartienne à aucun $\mathscr{C}_{A}$-circuit inclus dans c. Donc :

$$
\begin{aligned}
& x \in \varphi(A \cup(C-x)), \quad \text { mais } \quad x \notin \varphi_{A}(C-x) . \\
& \text { D' où }\left(a_{3}\right) \text { et }\left(a_{4}\right)
\end{aligned}
$$

$\begin{aligned} & \text { En général } \varphi(\mathrm{X}) \notin \varphi_{\mathrm{A}}(\mathrm{X}) \text {. On a d'ailleurs d'après }\left(\mathrm{a}_{4}\right) \text { : } \\ &\left(\varphi \subset \varphi_{\mathrm{A}}\right) \longleftrightarrow\left(\varphi \ll \varphi_{\mathrm{A}}\right) \Longleftrightarrow\left(\mathrm{X} \in \beta(\mathrm{E}), \varphi_{\mathrm{A}}(\mathrm{X})=\varphi(\mathrm{A} \cup \mathrm{X})\right)\end{aligned}$ 
Avant de démontrer $\left(a_{5}\right)$, il est bon d'énoncer les :

Corollaire 1.- Soient $M$ et $N$ deux parties libres d'un $\mathscr{Z}$-espace E. Les propriétés suivantes sont équivalentes :
a) $M \cup N \in \mathcal{L} \quad$ et $M \cap N=\varnothing$
b) $\mathrm{M} \in \mathscr{L}_{\mathrm{N}}$ et $\mathrm{N} \in \mathscr{L}$
c) $\mathrm{N} \in \mathscr{L}_{M}$ et $\mathrm{M} \in \mathscr{L}$

Corollaire 2.- Soit E un $\mathscr{L}$-espace, ACE et B une base de A. Pour toute partition $(M, N)$ de $B, M$ est une $\mathscr{L}_{N}$-base de A. Réciproquement, si $N$ est une partie libre de $A$ et $M$ une $\mathscr{L}_{N}$-base de $A, M \cap N=\emptyset$ et $M \cup N$ est une base de $A$.

Ces corollaires se démontrent sans difficultés à partir de la propriété $\left(a_{2}\right)$. Démontrons $\left(a_{5}\right)$ :

$$
\begin{gathered}
\forall x \in \mathcal{F}(\mathrm{E}) \quad, \quad \forall \mathrm{z} \in \mathcal{J}(\mathrm{A}) \cap \mathscr{L}, J \mathrm{Y} \subset \mathrm{X}, \quad \mathrm{Y} \in \mathscr{L}_{\mathrm{z}} \text { tel que : } \\
\mathbf{r}_{\mathrm{z}}(\mathrm{X})=\operatorname{Card} \mathrm{Y}=\mathrm{r}_{\mathrm{z}}(\mathrm{Y})
\end{gathered}
$$

et d'après le corollaire $1, \quad \mathrm{Y} \cap \mathrm{Z}=\emptyset$ et $\mathrm{Y} \cup \mathrm{Z} \in \mathcal{L}$, donc :

$$
\mathbf{r}(X \cup Z) \geq \operatorname{Card} Y+\operatorname{Card} Z=r_{Z}(X)+\mathbf{r}(z)
$$

soit

$$
r_{z}(x) \leq r(x \cup z)-r(z)
$$

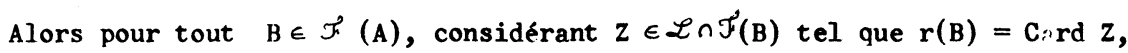
on a :

$$
r_{B}(X) \leq r_{Z}(X) \leq r(X \cup Z)-r(z) \leq r(B \cup X)-r(B)
$$

d'où

$$
\forall x \in \mathcal{F}(E) \quad, \quad r_{A}(X) \leq \operatorname{Min}(r(B \cup X)-r(B)) \quad[B \in \mathcal{F}(A)]
$$


(Il est aisé de vérifier que, si $\left.A \subset A^{\prime}, \quad r[A] \rightarrow r\left[A^{\prime}\right]\right)$.

$Z r(B \cup X)-r(B) \quad n$ 'est pas une fonction décroissante de $B$ en général.

Soit maintenant $(E, \mathscr{C})$ un $\psi$-espace. L'on va d'abord démontrer que $\mathscr{C}_{\mathrm{A}} \ll \mathscr{C}$ en prouvant par récurrence sur $\mathrm{Card}(\mathrm{C}-\mathrm{A})$ que tout $\mathrm{c} \in \mathscr{C}$ est réunion de $C_{A}$-circuits. C'est trivial si Card $(C-A)=0$. Supposons le vrai si Card $(C-A) \leqslant n-1$ et soit $c \in \mathcal{C}$ tel que Card $(C-A)=n$.

Si $\mathrm{C}-\mathrm{A} \epsilon \mathscr{C}_{\mathrm{A}}$, $\mathrm{c}$ est trivialement réunion de $\mathscr{C}$-circuit. Sinon $\mathrm{C}-\mathrm{A} \notin \mathscr{C}_{\mathrm{A}}$, donc : $\exists \mathrm{C}^{\prime} \in \mathscr{C}_{\text {tel }}$ que $\mathrm{C}^{\prime}-\mathrm{A} \underset{\mathrm{C}-\mathrm{A}}{ }$ et $\mathrm{C}^{\prime}-\mathrm{A} \in \mathscr{C}_{\mathrm{A}} \cdot$ Alors, $\forall x \in \mathrm{C}$, si $x \in \mathrm{C} \cap \mathrm{A},\{x\} \in \mathscr{E}_{\mathrm{A}} ;$ si $\mathrm{x} \in \mathrm{C}^{\prime}-\mathrm{A}, \mathrm{C}^{\prime}-\mathrm{A} \in \mathscr{C}_{\mathrm{A}} \quad ;$ si $\mathrm{x} \in \mathrm{C}-\mathrm{A}$ et $x \notin C^{\prime}-A$, il existe $a \in(C-A) \cap\left(C^{\prime}-A\right)=C \cap C^{\prime}-A$ et d'après l'axiome $\left(C^{\prime}\right)$, il

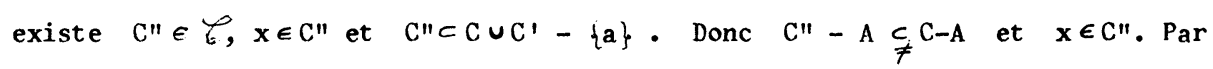
hypothèse de récurrence, $C "$ est réunion de $\mathscr{C}_{A}$-circuits, et en particulier il existe $\mathrm{c}_{\mathrm{x}} \in \mathscr{C}_{\Lambda}$ tel que $\mathrm{x} \in \mathrm{C}_{\mathbf{x}}$ et $\mathrm{C}_{\mathrm{x}} \subset \mathrm{C}^{\prime \prime}-\mathrm{A}$, donc $\mathrm{c}_{\mathbf{x}} \subset \mathrm{C}-\mathrm{A}$.

$$
\begin{aligned}
& \text { Comme } \quad \mathscr{C}_{\mathrm{A}} \ll \mathscr{C}, \text { d'après }\left(\mathrm{a}_{4}\right), \\
& \forall \mathrm{X} \in \mathrm{E}, \quad \varphi_{\mathrm{A}}(\mathrm{x})=\varphi(\mathrm{A} \cup \mathrm{X})
\end{aligned}
$$

et, $\varphi$ étant une fermeture, $\varphi_{A}$ est aussi une fermeture, donc $\left(E, \varphi_{A}\right)$ est un $\psi$-espace. On en déduit alors aisément le :

Corollaire 3.- Soit $(E, \varphi)$ un $\varphi$-espace et ACE. Alors $\left(E, \varphi_{A}\right)$ est un $\varphi$-espace, $\varphi \subset \varphi_{\mathrm{A}}$ et $\varphi_{\mathrm{A}}=\varphi_{\varphi(\mathrm{A})}$.

$A$ et $B$ étant deux parties de $E$, on a les propriétés :
a)
$\mathrm{A} \subset \mathrm{B}$
$\rightarrow \varphi_{\mathrm{A}}$
est fortement plus fine que $\varphi_{B}$
b)
$\varphi(A) \subset \varphi(B) \Longleftrightarrow \varphi_{A}$ est fortement plus fine que $\varphi_{B}$
c)
$\varphi(\mathrm{A})=\varphi(\mathrm{B}) \Longleftrightarrow \varphi_{\mathrm{A}}=\varphi_{\mathrm{B}}$ 
Ces propriétés sont fausses dans un $\mathscr{L}$-espace quelconque.

Soit $(E, \varphi)$ un $\varphi$-espace, $A \subset E$ et $X \in \Im(E)$. Si B est une base de $A$ et $B^{\prime}$ une base de BuX contenant B, alors B' est une base de AUX et, d'après les corollaires 1,2 et $3, B^{\prime}-B$ est une $\varphi_{A}$-base de $x$.

Donc : $\quad \mathbf{r}_{A}(X)=\operatorname{Card}\left(B^{\prime}-B^{\prime}\right)$

Si $r(A)$ est fini, on a $r_{A}(x)=r(X \cup A)-r(A)$

Sinon, B et B' sont infinies ; mais :

$\forall x \in X, \exists B^{\prime} x_{x} \in \mathcal{F}^{\prime}\left(B^{\prime}\right), \quad x \in \varphi\left(B^{\prime} x^{\prime}\right)$

donc $\quad B_{0}^{\prime}=U B^{\prime} \quad[x \in X] \in J^{\prime}\left(B^{\prime}\right)$ et $X \subset \varphi\left(B_{0}^{\prime}\right)$.

En outre $\forall x \in B^{\prime}-B, \quad x \in X$, donc $B^{\prime} x^{\prime}\{x\}: B^{\prime} \supset B^{\prime}-B$

Donc $B^{\prime}-B$ est encore une $\varphi_{B_{0}}$-base de $X$

où $B_{0}=B_{0}^{\prime}-\left(B^{\prime}-B\right) \in \mathcal{F}(B)$

et $\mathbf{r}_{B_{0}}(X)=\operatorname{Card}\left(B^{\prime}-B\right)=r_{A}(X)=r\left(X \cup B_{0}\right)-r\left(B_{0}\right)$

Donc

$$
r_{A}(X)=\operatorname{Min}(r(X \cup B)-r(B)) \quad\left[B \in F^{f}(A)\right]
$$

et le :

Corollaire $4 .-$ Soit $(E, \varphi)$ un $\varphi$-espace et $A \subset E$.

$$
\forall x \in \mathcal{B}(E), \quad \varphi_{A}(X)=\varphi(A \cup X)
$$

et

$\forall x \in \mathbb{F}^{(E)}, \quad$ si $r(A)$ est fini, $\quad r_{A}(X)=r(X \cup A)-r(A)$

sinon :

$$
r_{A}(X)=\operatorname{Min}(r(X \cup B)-r(B)) \quad[B \in \mathscr{H}(A)]
$$

où $r(X \cup B)-r(B)=r_{B}(X)$ est une fonction décroissante de $B$. 


\section{2.- Structures initiales dans les $\mathscr{L}$-espaces et dans les $\varphi$-espaces}

\subsection{1.- Images réciproques et structures induites}

Soit $E$ un ensemble, $\left(E^{\prime}, \mathscr{L}^{\prime}\right)$ un $\mathscr{L}^{\prime}$-espace et $f$ une application de $E$ dans $E^{\prime}$. La structure image réciproque par $f$ de $\left(E^{\prime}, \mathscr{L}^{\prime}\right)$ dans $L_{\text {est, }}$ si elle existe, le $\mathscr{L}$-espace $(E, \mathscr{L})$ tel que :

(I N ) Pour tout $\mathscr{L}^{\prime \prime}$-espace $\left(E^{\prime \prime}, \mathscr{L}^{\prime \prime)}\right.$, pour toute application $g$ de $E^{\prime \prime}$ dans $E$, $g$ est un $\underline{L}$-morphisme si et seulement si $f \circ g$ est un $\underline{L}$-morphisme.

Or $(E, \mathscr{Z})$ est la structure la moins fine sur $E$ telle que $f$ soit un morphisme, et $f$ est un morphisme si et seulement si, pour tout $x \in \mathcal{G}(E)$, $\mathbf{r}(\mathrm{X}) \geqslant \mathbf{r}^{\prime}(\mathbf{f}(\mathrm{X}))$.

Considérant alors $(E, \mathcal{L})$ telle que :

$$
\forall x \in \mathcal{F}(E) \quad, \quad \mathbf{r}(\mathrm{X})=\mathbf{r}^{\prime}(f(\mathrm{X}))
$$

on démontre aisément que :

a) si r' vérifie $\left(R_{1}\right) \quad\left(\operatorname{resp} .\left(R_{2}\right) ; \operatorname{resp} .\left(R_{3}\right)\right)$; il en est de même de $r$.

b) la propriété (I N) est réalisée par $(E, \mathscr{L})$

c) f est alors un $\underline{L}^{<}$-morphisme et (I N) est réalisée dans $\underset{L}{L}$.

On a donc ainsi la structure image réciproque dans $\underset{m}{L}$ et $\underset{m}{L}$, et a fortiori dans $\Phi$ et $\Phi^{<}$. D'où le :

Théorème 3 .-

Soit $\left(E^{\prime}, \mathscr{L}^{\prime}\right)$ un $\mathscr{L}^{\prime}$-espace, $E$ un ensemble et $f$ une application de $E$ dans E'. La structure image réciproque par $f$ de $\left(E^{\prime}, \mathcal{L}^{\prime}\right)$ dans $I_{\text {m }}$ existe toujours : c'est le $\mathscr{L}$-espace $(E, \mathscr{L})$ défini par $\mathcal{l}^{\prime} u n e$ des propriétés équivalentes : 
L-espaces - $\Phi$-espaces

55

$\left(I R_{1}\right) \quad(\forall x \in \beta(E)) \quad\left((x \in \mathcal{L}) \Longleftrightarrow\left((f(x))_{x \in X}\right.\right.$ est $\mathscr{L}^{\prime}-$ libre $\left.)\right)$

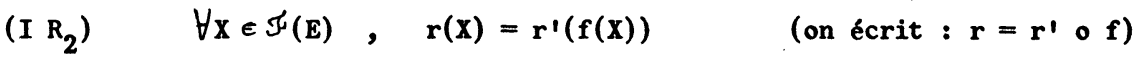

$\left(I_{3}\right) \quad \mathscr{C}=\{x \mid X \subset E, \quad f x$ injective et $f(x) \in \mathscr{C}\} \quad U \quad\{\{x, y\} \mid x \in E, y \in E$, $x \neq y$, et $\left.f(x)=f(y) \notin \varphi^{\prime}(\phi)\right\}$

$\left(I_{4}\right) \quad(\forall x \in \beta(E)) \quad\left((x \in B) \Leftrightarrow\left((f(x))_{x \in X}\right.\right.$ base de $\left.f(x)\right)$

$\left(\begin{array}{ll}\left(R_{5}\right.\end{array}\right) \quad \varphi=f^{-1} \circ \varphi \circ f$

et, pour les $\varphi$-espaces seulement :

$\left(\begin{array}{ll}I R_{6}\end{array}\right) \quad \mathscr{S}=\left\{\mathrm{f}^{-1}\left(\mathrm{~A}^{\prime}\right) \mid \mathrm{A}^{\prime} \in \mathscr{S}^{\prime}\right\}$

Alors $f$ est un $\underset{\text { mim }}{\mathfrak{L}}$-morphisme et $(E, \mathscr{L})$ est asssi la structure image réciproque de $\left(E^{\prime}, \mathscr{L}^{\prime}\right)$ par $f$ dans $\underline{L}^{<}$.

Si $\left(E^{\prime}, \mathscr{L}^{\prime}\right)$ est un $\varphi$-espace, $(E, \mathscr{L})$ est un $\varphi$-espace et $c^{\prime}$ est la structure image réciproque dans $\Phi_{\text {mom }}$ et dans $\underset{m \times}{\Phi}$.

Corollaire : Soit $(E, \mathscr{L})$ un $\mathscr{L}$-espace et $A \subset E$. La structure induite $\mathscr{L}_{\text {A }}$ de $(E, \mathcal{L})$ sur A existe toujours et on a (*) :

$$
\left.\mathscr{L}\right|_{A}=\mathscr{L} \cap \mathscr{F}_{(A)} \quad ;\left.\quad r\right|_{A}=\left.r\right|_{F(A)} ; \varphi_{\left.\right|_{A}}=\ell_{n} F_{(A)}
$$

$\left.{ }^{B}\right|_{A}$ est 1 'ensemble des bases de $A$.

et $\forall \mathbf{X} \in \beta(\mathrm{A}),\left.\quad \varphi\right|_{\mathrm{A}}(\mathrm{X})=\varphi(\mathrm{X}) \cap \mathrm{A}$.

Si $(E, \mathscr{L})$ est un $\varphi$-espace, $\left(A,\left.\mathscr{L}\right|_{A}\right)$ est un $\varphi$-espace (on a la même structure induite pour $\mathrm{L}, \mathrm{L}_{m}^{<}$et éventuellement $\Phi$ et $\Phi^{<}$).

(*) Une convention usuelle consiste à écrire $\left.f\right|_{X}$ la restriction de $f$ à $X$. 


\subsection{2. - Existence de structures initiales quelconques.}

Soit $\left(E_{i}, \mathscr{L}_{i}\right)$ une famille de $\mathscr{L}$-espaces, $E$ un ensemble et pour tout $i, f_{i}$ une application de $E$ dans $E_{i} \cdot$ Il est aisé de démontrer que la

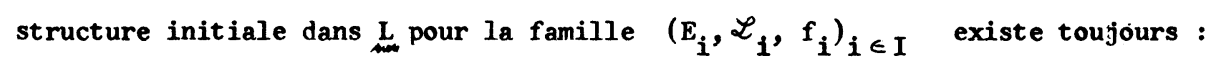
c'est (E, $\left.\underset{i}{\mathrm{M} x} \mathbf{r}_{i} \circ f_{i}\right)$

Les théorèmes 1 et 3 permetterit de calculer $\mathscr{L} \ldots$

Cependant, en général, les structures initiales n'existent plus dans $\underset{m}{L^{<}}, \underset{m}{\Phi}$ et $\Phi_{m}^{<}$, cependant, la famille $\left(E_{i}, \mathscr{L}_{i}, f_{i}\right)$ étant fixée, on a toujours avantage à la résoudre d'abord dans $\underset{\mathrm{m}}{\mathrm{L}}$. Si l'on obtient une structure de $\varphi$-espace et si les $f_{i}$ sont des $\Phi\left(\right.$ resp. $\left.\Phi^{<}\right)$-morphismes, on a alors la structure initiale dans $\Phi($ resp. $\Phi)$.

\subsection{3.- Structures produits}

La structure produit existe toujours dans $\underset{\mathrm{I}}{\mathrm{L}}$ mais rarement dans $\Phi$. Voici une étude de la structure produit dans $\Phi^{<}$.

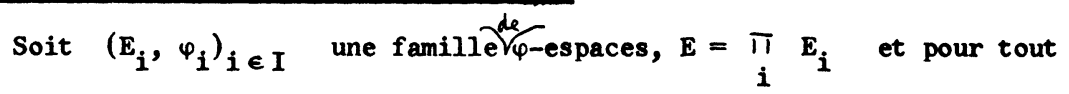
$i \in I, \quad p_{i}$ la projection de $E$ sur $E_{i}$.

$$
\mathrm{pr}_{i} \text { est un morphisme de }(E, \varphi) \text { dans }\left(E_{i}, \varphi_{i}\right) \text { pour tout } i \in I \text { si : }
$$

$$
\forall x \in \beta(E), \varphi(x) \subset \bigcap_{i} p_{i}^{-1} \circ \varphi_{i} \circ p_{i}(x)=\sigma(x)
$$

donc

$$
\sigma(x)=\prod_{i \in I} \varphi_{i} \circ \operatorname{pr}_{\mathbf{i}}(x)
$$

$\sigma$ vérifie les axiomes $\left(G_{2}\right)$ et $\left(G_{6}\right)$, mais elle ne vérifie $\left(G_{1}\right) \quad\left(\right.$ resp. $\left.\left(G_{3}\right)\right)$ que si et seulement si tous les $\varphi$-espaces $\left(E_{i}, \varphi_{i}\right)$, sauf un nombre fini (resp. sauf un au plus), vérifient la propriété :

$$
\forall x \in E_{i}, \quad \varphi_{i}(x)=E_{i}
$$


Donc, sauf pour des cas triviaux, $\quad \sigma \notin \Phi(E)$.

Soit maintenant $(E, \varphi)$ un $\varphi$-espace tel que :

pour tout $\varphi$ !-espace $\left(E^{\prime}, \varphi^{\prime}\right)$, pour toute application $g$ de $E^{\prime}$ dans $E$, pour tout $i \in I, \quad \operatorname{pr}_{i} \circ \mathrm{g}$ soit un morphisme. Alors :

$$
g \circ \varphi^{\prime} \quad C \quad \sigma \circ g
$$

donc, si $\sigma \in \Phi(E),(E, \sigma)$ est la structure produit des $\left(E_{i}, \varphi_{i}\right)$;

mais si $\sigma \notin \Phi_{m}(E)$, même si la borne inférieure des $\varphi \in \underset{m}{\Phi}(E)$ telles que $\varphi \subset \sigma \quad$ existe, la structure produit n'existe pas en général.

\section{3.- Structures finales dans les $\mathscr{L}$-espaces et dans 1 es $\varphi$-espaces}

\subsection{1.- Images directes et structures quotients.}

L'on démontre comme ci-dessus que, dans $\underset{\mathrm{m}}{\mathrm{L}}$, la structure image directe de $(E, \mathscr{L})$ par une application $f$ de $E$ dans $E^{\prime}$ existe toujours ; $c^{\prime}$ est $\left(E^{\prime}, \mathscr{L}^{\prime}\right)$ ainsi définie :

$$
\begin{aligned}
& \mathscr{C}^{\prime}=\left\{f(c) \mid c \in \mathscr{C} \text { et } f_{\mid c} \text { injective }\right\} \\
& \text { Cependant, si }(E, \mathscr{L}) \text { est un } \varphi \text {-espace, }\left(E^{\prime}, \mathscr{L}^{\prime}\right) \text { n'est pas un }
\end{aligned}
$$

$\varphi$-espace en général.

D'où de même la structure quotient dans $\underset{\operatorname{man}}{\mathrm{L}}(\mathrm{E})$.

Par contre dans $\underset{. m}{L_{n}^{<}}, \Phi$ et $\underset{m}{\Phi}$ les structures images directes et quotient n'existent pas en général. 


\subsection{2.- Existences de structures finales quelconques}

Soit $\left(E_{i}, \mathscr{L}_{i}\right)$ une famille de $\mathscr{L}$-espaces, $E$ un ensemble et pour tout $1, g_{i}$ une application de $E_{i}$ dans $E$. Il est aisé de démontrer que la structure finale dans $\underset{w}{L}$ pour la famille $\left(E_{i}, \mathscr{L}_{i}, g_{i}\right)$ existe toujours : c'est $\left(\mathrm{E}, \wedge \mathscr{C}_{i}^{0}\right)$

$$
\text { où } \begin{aligned}
\forall i \in I, \quad \mathscr{C}_{i}^{0} & =\left\{g_{i}(c) \mid c \in \mathscr{C}_{i} \text { et } g_{i} \mid c \text { injective }\right\} \\
\text { et } & \wedge \mathscr{C}_{i}^{0}=\operatorname{MIN}\left(\underset{i}{\operatorname{C}} \mathscr{C}_{i}^{0}\right) .
\end{aligned}
$$

Cependant, en général, les structures finales n'existent plus dans $L^{<}$, $\Phi$ et $\Phi^{<}$et l'on a des propriétés analogues à celles de 2.2 .2 .

\subsection{3.- Sommes directes et unions directes.}

Si f est une application injective, la structure image directe d'un $\varphi$-espace par $f$ est un $\varphi$-espace ( $I^{\prime}$ axiome $\left(c_{2}\right)$ "se transporte"), ce qui n'est plus vrai en général quand $f$ 'est pas injective.

D'une manière générale, soit $\left(E_{i}, \mathscr{L}_{i}\right)$ une famille de $\mathscr{L}$-espaces, $E$ un ensemble, et pour tout $i, g_{i}$ une injection de $E_{i}$ dans $E$, de sorte que

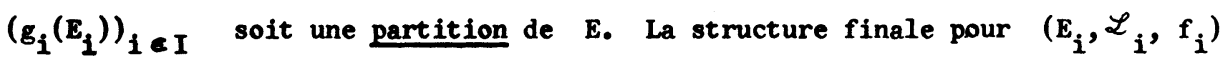
dans $\mathcal{L}$ est appellée structure somme directe des $\mathcal{L}_{i}$ et $I^{\prime}$ 'on écrit $\underset{i}{\oplus} \mathscr{L}_{i}$. On a $\underset{i}{\oplus} \mathscr{C}_{i}=\left\{g_{i}(\mathrm{c}) \mid \mathrm{c} \in \mathscr{C}_{i}\right.$ et $\left.i \in I\right\}$

Toute somme directe de $\varphi$-espace est un $\varphi$-espace.

Si les $E_{i}$ sont des parties de $E$ et $f_{i} l$ 'inclusion canonique de $E_{i}$ dans $E$, alors $\oplus \mathscr{L}_{i}$ est appellée $I^{\prime}$ union directe des $\left(E_{i}, \mathscr{L}_{i}\right)$ et l'on écrit $\uplus_{i} \mathscr{L}_{i}{ }^{i}$. On a :

$$
\biguplus_{i} \quad \mathscr{C}_{i}=\bigcup_{i} \mathscr{C}_{i} \quad \text { (réunion dans } \beta(\beta(E)) \text { ) }
$$


L'on démontre alors aisément le :

\section{Théorème 4.-}

Soit $\left(E_{i}\right)_{i \in I}$ une partition d'un $\mathscr{L}$-espace $(E, \mathscr{L})$ et pour tout $i \in I, \quad\left(E_{i}, \mathcal{L}_{i}\right)$ structure de $\mathscr{L}$-espace sur $E_{i}$. Alors les propriétés suivantes sont équivalentes :

(U D )

$$
E=\biguplus_{i} E_{i}
$$

$\left(\begin{array}{ll}U & D_{1}\end{array}\right)$

$\mathscr{L}=\{\mathrm{x} \mid \mathrm{x} \subset \mathrm{E}$

et $\left.\forall i \in I, \quad x \cap E_{i} \subset \mathscr{L}_{i}\right\}$

$\left(\begin{array}{ll}U & D_{2}\end{array}\right)$ $\forall \mathrm{x} \in \mathscr{H}(\mathrm{E}) \quad, \quad \mathrm{r}(\mathrm{x})=\sum \mathrm{r}_{\mathbf{i}}\left(\mathrm{X} \cap \mathrm{E}_{\mathbf{i}}\right) \quad[\mathrm{i} \in \mathrm{I}]$

$\left(\begin{array}{ll}U & D_{3}\end{array}\right)$ $\mathscr{C}=\bigcup_{i} \mathscr{C}_{i}$

$\left(\begin{array}{ll}U & D_{4}\end{array}\right)$ $x \in \beta \quad$ si et seulement si pour tout $i \in I, \quad x \cap E_{i} \in \not_{i}$

$\left(\begin{array}{ll}( & D_{5}\end{array}\right)$ $\forall x \in \beta(E), \varphi(X)=\bigcup_{i} \varphi_{i}\left(X \cap E_{i}\right)$

En outre $(E, \mathscr{L})$ est un $\varphi$-espace si et seulement si tous les $\left(E_{i}, \mathscr{L}_{i}\right)$ sont des $\varphi$-espaces.

Lors de la disjonction, on verra des propriétés plus précises, notamment pour les $\varphi$-espaces (voir chapitre III).

2.4.- Irréductibilités. Isomorphismes.

L'existence de structures image réciproque et somme directe permet de réduire un "$\varphi$-espace" de deux manières : 
2.4.1. Première reduction associée d̀ l'image réciproque.

La relation $"(E, \varphi) \propto\left(E^{\prime}, \varphi^{\prime}\right)$ si et seulement si il existe une application $f$ de $E$ dans $E^{\prime}$ telle que $\varphi$ soit $l^{\prime}$ image réciproque par $f$ de $\psi^{n}$ est une relation de préordre sur les $\varphi$-espaces.

Un $\varphi$-espace $(E, \varphi)$ est dit 1 -réduit si tout $\varphi$-espace $\left(E^{\prime}, \varphi^{\prime}\right)$ tel que $\left(E^{\prime}, \varphi^{\prime}\right) \propto(E, \varphi)$ est isomorphe à $(E, \varphi)$.

Proposition 2.- Un $\varphi$-espace $(E, \varphi)$ est 1-réduit si et seulement si il vérifie l'une des propriétés équivalentes (donc toutes):

(1) $\varphi(\varnothing)=\varnothing \quad$ et $\varphi(x)=\{x\}$ pour tout $x$

(2) tout $X \subset E$ tel que Card $X \leq 2$ est $\varphi$-libre

(3) Les circuits de $E$ ont tous au moins trois éléments.

Il est aisé de vérifier l'équivalence des propriétés (1), (2) et (3), et un $\varphi$-espace qui les vérifie est 1-réduit. L'on obtient ainsi tous les $\varphi$-espaces 1-réduits. En effet l'on va définir le foncteur de 1-réduction qui :

- à tout $\varphi$-espace $(E, \varphi)$ non grossier associe canoniquement son 1-réduit $\left(E_{q}, \varphi_{1}\right)$ tel que $\left(E_{1}, \varphi_{1}\right) \propto(E, \varphi)$

- à tout morphisme $f$ de $(E, \varphi)$ dans $\left(E^{\prime}, \varphi^{\prime}\right)$ tel que $f^{-1}\left(\varphi^{\prime}(\varnothing)\right)=\varphi(\varnothing) \underset{\neq E}{\subset E} \quad$ associe un morphisme $f_{1}$ de $\left(E_{1}, \varphi_{1}\right)$ dans $\left(E^{\prime}, \varphi^{\prime}{ }_{1}\right)$. Soit $\underset{m}{T}$ la catégorie dont les objets sont les treillis géométriques, les morphismes de $T$ dans $T$ ' étant les applications $f$ de $T$ dans $T$ ' telles que, pour toute famille $\left(x_{i}\right)$ d'éléments de $T$,

$$
f\left(\vee x_{i}\right)=\vee f\left(x_{i}\right) \quad \text { et } \quad f\left(\wedge x_{i}\right)=\wedge f\left(x_{i}\right)
$$

et, $u$ étant le plus grand élément de $T, f(u)$ est le plus grand élément de $T$ '. 
$F:(E, \varphi) \longrightarrow T(E, \varphi)$ treillis géométrique des sous-espaces de $(E, \varphi)$ et $\left(f:(E, \varphi) \longrightarrow(F, \psi) \longrightarrow\left(" f^{-1} \|: T(F, \psi) \longrightarrow T(E, \varphi)\right)\right.$ est un foncteur de $\underset{m}{\Phi_{m}^{<}}$dans $\underset{m}{T *}$ (catégorie duale de $\underset{m}{T}$ ) (voir 2.1.1. $\left.\left(\mathrm{h}_{1}{ }^{<}\right) \Leftrightarrow\left(\mathrm{H}_{5}{ }^{<}\right)\right)$.

Soit $\underset{\text { min }}{T_{1}}$ la sous-catégorie de $\underset{m}{T}$ dont les objets sont les treillis géométriques ayant au moins deux éléments et dont les morphismes transforment le plus petit élément en le plus petit élément.

G : $\quad T \longrightarrow(P(T), \varphi)$ où $P(T)$ est l'ensemble des points (*) de $T$ et où, pour tout $X \subset P(T), \varphi(X)$ est $I$ 'ensemble des points $X \leq \vee X$ et

$$
\left(\mathrm{u}: \mathrm{T}^{\prime} \longrightarrow \mathrm{T}\right) \longrightarrow\left(\mathrm{G} u: \mathbf{x} \longrightarrow \mathrm{Gu}(\mathbf{x})=\wedge A^{\prime} \quad\left[A^{\prime} \in \mathrm{T}^{\prime} \text { et } u\left(A^{\prime}\right) \geq \mathbf{x}\right]\right)
$$

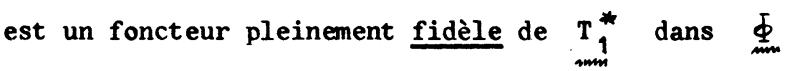

$$
\mathrm{G}\left(\mathrm{T}_{1}^{*}\right) \subset \underset{\Phi_{1}}{\Phi_{1}} \text { sous-catégorie pleine de } \underset{m}{\Phi^{<}} \text {dont les objets sont les }
$$
$\varphi$-espaces 1-réduits.

En effet $(P(T), \varphi)$ est 1-réduit, et, si $(E, \varphi)$ est 1-réduit, $G \circ F(E, \varphi)=\left(E_{1}, \varphi_{1}\right)$ où $E_{1}=\{\{x\} \mid x \in E\}$ et $x \rightarrow\{x\}$ est un isomorphisme de $E$ sur $E_{1}$. Donc $(E, \varphi)$ est objet de $\Phi_{1}$ si et seulement si tout $x \in E$ est de la forme $x=\left\{x_{0}\right\}$. Si l'on admet l'axiome de fondation (voir[Q], chapitre I), l'on sait que, en général, il existera des éléments $x \in E$ tels que aucun élément ne leur appartienne.

(*) Les points d'un treillis $T$ ayant un pius petit élément 0 sont les éléments $x$ de $T$ tels que $x>0$ et il $n^{\prime}$ existe aucun élément $y \in T$ tel que $0<y<x$. 
Soit $\underset{m m}{\Phi^{\prime<}}$ la sous-catégorie de $\underset{m}{ } \Phi^{<}$

- dont les morphismes sont les morphismes non triviaux, i.e. les morphismes $f$ de $(E, \varphi)$ dans $(F, \psi)$ tels que $f(E) \notin \psi(\varnothing)$. (Alors les $\varphi$-espaces grossiers sont isolés et perdent leurs unités)

- et dont les objets sont les $\varphi$-espaces non grossiers; et $\underset{m}{\Phi}$ la sous-catégone propre de $\Phi_{m}^{\prime<}$ dont les morphismes sont les morphismes exacts, i.e. les morphismes $f$ de $(E, \varphi)$ dans $(F, \psi)$ tels que $f^{-1}(\psi,(\varnothing))=\varphi(\varnothing)$. $\underset{m m}{\Phi_{1}}$ est une sous-catégorie pleine de $\Phi_{m}^{\prime<}$ et $\underset{m m}{\Phi^{e}}$

A tout morphisme non trivial $f$ de $(E, \varphi)$ dans $(F, \psi)$ est associé le morphisme exact $p(f)$ de $\left(E, \varphi\left[f^{-1}(\psi(\varnothing))\right]\right)$ dans $(F, \psi)$ ayant même application sous-jacente, et, posant $p(E, \varphi)=(E, \varphi), \quad$ e est une projection de ${ }^{\prime<}$ sur $\Phi^{e}$, mais ce n'est pas un foncteur.

\section{L'on démontre alors aisément que : \\ $F_{1}=G \circ F$, foncteur de $\underset{m n}{\Phi^{e}}$ dans $\Phi_{1}$ est le foncteur de} 1-réduction :

a) G OF $(E, \varphi)=\left(E_{1}, \varphi_{1}\right)$ où :

$$
\begin{aligned}
E_{1} & =\{\varphi(\mathbf{x}) \mid \mathbf{x} \in \mathbf{E}-\varphi(\phi)\} \\
\pi: x & \rightarrow \varphi(x) \quad \text { application de } E_{-}-\varphi(\phi) \text { sur } E_{1} \ldots \text { est la projection }
\end{aligned}
$$

canonique de E sur son 1-réduit,$\varphi_{1}$ est 1 'image directe de $(E-\varphi(\phi), \varphi$ induit $)$ par $\pi$ et

$$
\varphi_{1}=\pi \circ \varphi \circ \pi^{-1}
$$

En outre, il existe au moins une application $f$ de $E_{1}$ dans $E$ telle que $f \circ \pi={ }^{1} E_{1} \quad(f$ est une section de $\pi$ ) et toute section, $f$ est telle que $\left(E_{1}, \varphi_{1}\right)$ soit l'image réciproque de $(E, \varphi)$ par $f$. 
b) $\left[u:(E, \varphi) \longrightarrow\left(E^{\prime}, \varphi^{\prime}\right)\right] \longrightarrow G \circ F(u)=u_{1}$

est tel que le diagramme ci-contre soit commutatif. Plus précisément

$u_{1}=\pi^{\prime} \circ u_{0} \circ \pi^{-1}$

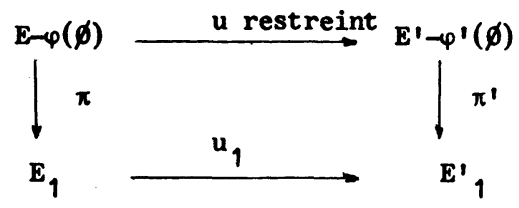

c) tout morphisme non trivial f est associé le morphisme 1-réduit $f_{1}=G \circ F \circ P(f) \quad$ (mais ce procédé n'est plus fonctoriel).

d) Réciproquement, soient $(E, \varphi)$ et $\left(E^{\prime}, \varphi^{\prime}\right)$ deux $\varphi$-espaces. Si $\varphi(\varnothing) \neq \varnothing$ et $\varphi^{\prime}(\phi)=\varnothing$, il $n^{\prime}$ existe aucun morphisme de $(E, \varphi)$ dans $\left(E^{\prime}, \varphi^{\prime}\right)$.

Sinon, à toute application $\sigma_{0}$ de $\varphi(\varnothing)$ dans $\psi(\varnothing)$, à tout morphisme $u_{1}$ de $\left(E_{1}, \varphi_{1}\right)$ dans $\left(F_{1}, \psi_{1}\right)$, et à toute famille $\left(\sigma_{\mathbf{x}}\right)_{x \in E_{1}}$ diapplications $\sigma_{x}$ de $x-\varphi(\phi)$ dans $u(x)-\varphi^{\prime}(\phi)$, correspond le morphisme exact de $(E, \varphi)$ dans $(F, \psi)$ :

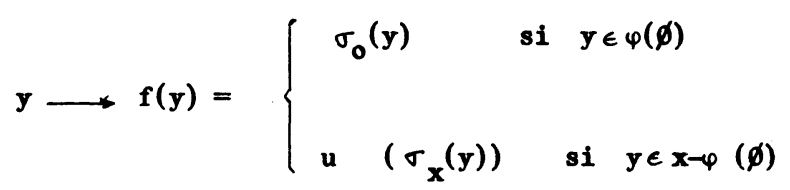

2.4.2.- Réduction associée à la somme directe (L्L -espaces)

Un $\mathscr{L}$-espace $(E, \mathscr{L})$ est dit $\underline{\text { 2-irréductible }}$ s'il n'existe pas de partition de $E$ en deux parties $A$ et $B$ telle que $(E, \mathcal{L})$ soit union directe de

$$
\left(A,\left.\mathscr{L}\right|_{A}\right) \text { et }\left(B, \mathscr{L}_{B}\right) \text {. }
$$

Un $\mathscr{Z}$-espace 2 -irréductible est dit $\underline{\text {-réduit }}$

Un $\varphi$-espace est dit 1-2-réduit s'il est à la fois 1-réduit et 2-réduit. 
Soit la relation d'équivalence $R$ sur le $\mathcal{L}$-espace $E:$
$R\left\{x, y \xi(x-y)\right.$ ou $\left(\begin{array}{l}i l \text { existe } n \text { circuits } C_{i} i=1, \ldots, n \text { de } E \text { tels que : } \\ x \in C_{1}, C_{i} \cap C_{i+1} \neq \emptyset \text { pour } i=1, \ldots, n-1 \text { et } y \in C_{n}\end{array}\right)$

Soit $E / R=I$ et $\left(E_{i}\right)_{i \in I}$ les classes d'équivalence de $E$ suivant

R. Alors $\left(E_{i}\right)$ est une partition de $E$, et, si pour tout $i \mathscr{C}_{i}=\mathscr{C}_{\cap} \mathfrak{F}_{\left(E_{i}\right) \text {, }}$ on a :

$$
\mathscr{C}=\bigcup_{i \in I} \mathscr{C}_{i}
$$

Donc $\left.\quad E=\biguplus_{i}\left(E_{i}, \mathscr{C}_{i}\right)=\biguplus_{i}\left(E_{i}\right), \varphi \mid E_{i}\right)$

et la :

proposition 3.- On appelle composantes d'un $\mathscr{L}$-espace $(E, \mathscr{L})$ les clasees d'équivalence $\left(E_{i}\right) \quad[i \in E / R]$ de $E$ suivant la relation d'équivalence $R$. Alors $E$ est union directe de ses composantes $E_{i}$ munies de $\mathcal{L}$ induit et chaque composante est 2-irréductible.

En particulier :

a) $\mathbf{E}_{\mathbf{i}}=\{\mathbf{x}\}$ si et seulement si $\mathbf{x} \in \varphi(\varnothing)$ ou $\mathbf{x}$ n'appartient à aucun circuit.

b) un $\mathscr{L}$-espace est 2-irréductible si et seulement si on peut joindre deux éléments quelconques de E par une suite finie (non vide) de circuits consécutivement non disjoints.

c) un espace vectoriel, un corps $K$ algébriquement clos (degré de transcendance) sont des $\varphi$-espaces irréductibles quand on leur enlève $\varphi(\varnothing)$. 


\subsubsection{Isomorphismes}

Les catégories $\underset{\operatorname{m}}{\mathrm{L}}$ et $\underset{\mathrm{m}}{\mathrm{L}}=$ (resp. $\Phi$ et $\Phi^{<}$) ont les mêmes isomorphismes : Une application $f$ de $(E, \mathscr{L})$ dans $\left(E^{\prime}, £\right)$ est un isómorphisme si et seulement si f est une bijection et vérifie l'une des propriétés équivalentes suivantes :

(1) $\left(\mathrm{x} \in \mathcal{L}^{\prime}\right) \Longleftrightarrow\left(\mathrm{f}(\mathrm{x}) \in \mathscr{L}^{\prime}\right)$

(2) $(x \in B) \Longleftrightarrow\left(f(x) \in B^{\prime}\right)$

(3) $(x \in \mathscr{C}) \Longleftrightarrow\left(f(x) \in \mathscr{C}^{\prime}\right)$

(4) $\forall x \in \beta(E), \quad f \circ \varphi(x)=\varphi^{\prime} \circ f(x)$

et, pour les $\varphi$-espaces seulement :

(5) $(A \in T(E, \varphi)) \Longleftrightarrow\left(f(A) \in T\left(E^{\prime}, \varphi^{\prime}\right)\right)$

Voici un résultat plus fin :

Un $\mathscr{L}$-espace est dit complet si toute partie libre finie est incluse dans un circuit.

Les $\mathscr{L}$-espaces complets sont les $\mathscr{L}$-espaces maximaux pour la relation d'ordre :

$$
(E, \mathscr{L}) \leq\left(\mathrm{E}, \mathscr{L}^{\prime}\right) \Longleftrightarrow \mathscr{C} \subset \mathscr{C}^{\prime}
$$

(L'ensemble $\Phi(E)$ des structures de $\mathscr{L}$-espace sur $E$ est alors un ensemble ordonné inductif).

exemple : Un espace vectoriel, un A-module sont des $\mathscr{L}$-espaces complets.

Si $(E, \mathscr{L})$ est un $\mathscr{L}$-espace complet, et si $f$ est une bijection de E $\operatorname{sur}\left(E^{\prime}, \mathscr{L}^{\prime}\right), \quad f$ est un isomorphisme si et seulement si on a :

(3c) $\quad(\mathrm{x} \in \mathscr{C}) \quad \Longrightarrow \quad(\mathrm{f}(\mathrm{x}) \in \mathscr{C})$ 
Cette propriété est l'analogue de théorèmes classiques en algèbre et en analyse : Si $f$ est une bijection linéaire, son inverse est linéaire ; Si $f$ est une bijection linéaire continue, son inverse est linéaire continue ( $s i$ on a suffisamment de propriétés sur les espaces topologiques considérés).

\section{5.- Le problème de la représentation}

On appelle représentation de $(E, \mathscr{L})$ dans $\left(E^{\prime}, \mathscr{L}^{\prime}\right)$ toute injection $f$ de $E$ dans $F$ telle que, $f$ restreint à $f(E)$ soit un isomorphisme de $(E, \mathscr{L})$ sur $\left(f(E), \mathcal{L}^{\prime} \mid f(E)\right)^{\prime}$

$L^{\prime}$ 'on dit encore que $\underline{f}$ est un isomorphisme de $(E, \mathcal{L})$ dans $\left(E^{\prime}, \mathscr{L}^{\prime}\right)$.

Etant donnée une classe $\mathrm{C}$ de $\mathscr{L}$-espace (resp. $\varphi$-espaces)

10) A quelle condition tout $\mathscr{L}$-espace (resp. $\varphi$-espace) se représente-t-il dans au moins un objet de C ?

20) Quels sont les $\mathscr{L}$-espaces (resp. $\varphi$-espaces) qui se representent comme parties d'un objet de C? Les caractériser de manière aussi simple que possible.

Voici quelques réponses dans le cas des $\varphi$-espaces, quand $\mathrm{C}$ est la classe des espares vectoriels.

a) Si $(E, \varphi)$ se reprèsente dans un espace vectoriel $F$ sur $k$, alors $(E, \varphi)$ se représente dans tout espace vectoriel $F^{\prime}$ sur $k^{\prime}$ tel que $\operatorname{dim} F^{\prime} \geq r(E)$ et $k^{\prime}$ contienne un sous-corps isomorphe à $k$, donc en particulier se represente dans $k^{(r(E))}$ espace vectoriel libre engendré par $r(E)$ sur $k$. On dira alors que $(E, \varphi)$ est k-représentable.

b) Soit $E$ un $\varphi$-espace tel que $\operatorname{Card} \varphi(\phi) \leq 1$. Si E est k-représentable, le $\varphi$-espace 1-réduit $E_{1}$ associé à $E$ est k-représentable. Inversement si $E_{1}$ 
est $k$-représentable, alors $E$ est $k^{\prime}$-représentable pour tout corps $k^{\prime}$ contenant un corps isomorphe à $k$ et tel que :

$$
\text { Card } k^{\prime} \geqslant a
$$

où

$$
a=(\operatorname{Sup}(\operatorname{Card} \varphi(\mathbf{x})-\varphi(\phi) \quad[\mathbf{x} \in \mathbf{E}])+1
$$

(ceci résulte de 2.4.1., d) en prenant pour $\sigma_{\mathbf{x}}$ des injections), 1e 1-réduit associé à un espace vectoriel est l'espace projectif de ses sous-espaces vectoriels de dimension 1).

c) Tout $\varphi$-espace $E$ tel que Card $\varphi(\varnothing) \leqslant 1$ et tel que $T(E, \varphi)$ soit une géométrie affine $(*)$ de dimension $\geqslant 3$ ou une géométrie plane affine arguesienne est k-représentable si et seulement si $k$ contient un sous-corps isomorphe à un corps $k_{0}$ défini intrinsèquement à partir de $T(E, \varphi)$ et

$$
\text { Card } k \geqslant a \quad \text { défini par (1). }
$$

(ce dernier point est une conséquence de [E], III, chapitre v, théorème 6, page 339 et chapitre VI, théorème 6, page 366). De même, de [E], III, chapitre VI, th. 9, p. 370 et de $[F]$, chapitre 17, pages $366-375$, il résulte un énoncé analogue à c) pour les géométries projectives irréductibles de dimension $\geq 3$ ou planes arguesiennes.

Il est aisé de construire des exemples simples de $\varphi$-espaces qui ne peuvent se représenter dans un espace vectoriel en contredisant par exemple le théorème de Desargues.

(*) Au sens de [E], III, chapitres IV et V . 
Le problème de représentation est ainsi ramené à un problème sur les 1-réduits. Il est aisé de le ramener à un problème sur les 1-2-réduits. Ce problzme a fait l'objet de nombreuses recherches. Une bibliographie a été faite, dont roici quelques exemples :

[A] Artin E. est excellent sur les géométries affines et projectives.

[E] III déjà cité

[F] chapitre 17, pp. $311-420$ est ce qu'il y a de mieux sur les géométries planes projectives et les liens entre la notion de géométrie plane et la notion de groupe.

[4] est une synthose de propriétés concernant les treillis et plus spécialement les géométries. A noter au paragraphe 7 un exposé des problèmes résolus et des questions ouvertes, ainsi que de divers procédés mis en oeuvre pour les résoudre, la plupart concernant le problème de la représentation. 
Initialement, l'un des buts de l'étude des $\varphi$-espaces a été de généraliser la notion de disjonction connue pour les parties d'un ensemble (famille de parties disjointes deux à deux) et pour deux corps intermédiaires (disjonction linéaire ou algébrique sur un corps de base), tout en gardant les notions de base, de dimension, ...

Rappelons qu'étant donné un corps $k$, un surcorps $K \epsilon_{2} \quad x$ corps intermédiaires $\mathrm{E}$ et $\mathrm{F}$, on dit que $\mathrm{E}$ et $\mathrm{F}$ sont linéairement (resp. faiblement linéairement ; resp. algébriquement) disjoints sur $k$ si :

$$
\forall \mathbf{X} \in \mathcal{B}(\mathbf{E}), \forall \mathbf{Y} \in \mathcal{Q}(\mathbf{F})
$$

$X$ et $Y$ étant des parties libres de $K$ considéré comme espace vectoriel sur $\mathbf{k}$<smiles>[Y][Y]([X])=[V]</smiles>
(resp.: idem ; resp.: des parties algébriquement libres sur k),

On a : $X$ est une partie libre de $K$, espace vectoriel sur $F$ et $Y$ est une partie libre de $K$, espace vectoriel sur $E$ (resp. $X \cap Y=\varnothing$ et $X \cup Y$ est une partie libre de $K$, espace vectoriel sur $k$ ) (resp. $X \cap Y=\varnothing$ et $X \cup Y$ est algébriquement libre sur $k$ ).

En $3.1,3.2$ et 3.3 est présentée une théorie de la disjonction qui contient les disjonctions ensembliste, faiblement linéaire et algébrique ; et en 3.4 une théorie de la disjonction double, qui contient la disjonction linéaire. 


\section{1.- Définitions et propriétés générales}

\subsection{1.- Définitions et notations.}

Les notations suivantes seront couramment utilisées :

Soit $\left(x_{i}\right)_{i \in I}$ une famille de parties d'un ensemble E. Pour tout $J \in I$, on écrit $X_{J}=U X_{i}[i \in J]$. On écrit $\left(Y_{i}\right) \subset\left(X_{i}\right)$ l'ordre produit sur $\not 3(E)$ et on l'appelle encore inclusion :

$\left(Y_{i}\right)$ est incluse dans $\left(X_{i}\right)$ si et seulement si, pour tout $i \in I, \quad Y_{i}<X_{i}$.

Définition 1.- Une famille $\left(x_{i}\right)_{i \in I}$ de parties d'un $\mathscr{L}$-espace $E$ est dite disjointe (ou $\mathscr{L}$-disjointe) si pour toute famille $\left(Y_{i}\right)$ incluse dans $\left(X_{i}\right)$ telle que pour tout $i \in I, Y_{i}$ soit $\mathscr{L}$-libre, on a la propriété :

(D) $Y_{I}$ est $\mathscr{L}$-libre et les $Y_{i}$ sont disjoints deux deux

On dit encore que les $x_{i}$ sont $\mathscr{L}_{\text {-disjoints, et, quand }}$ $I=\{1, \ldots, n\}$ est fini, que $x_{1}, \ldots, x_{n-1}$ et $x_{n}$ sont $\mathscr{L}$-disjoints.

Exemples :

1) Soit $\left(M_{i}\right)_{i \in I}$ une famille de sous-A-modules d'un $A-$ module $M$ (voir 1.2.1. pour $\mathscr{L}_{0}$ et $\left.\mathscr{L}_{1}\right)$.

1.a $\left(M_{i}\right)$ est $\mathscr{L}_{0}$-disjointe si et seulement si le sous-module $N$ engendré par les $M_{i}$ est (isomorphe à la) sonme directe (A-modules) des $M_{i}$ 
2) Soient $\left(A_{i}\right)_{i \in I}$ une famille de sous-anneaux d'un anneau commutatif et unitaire $A$ et $B$ un sous-anneau de $A$

2.a $\left(A_{i}\right)_{i \in I}$ est $\mathscr{L}_{0}$-disjointe si et seulement si les $A_{i}$ sont algébriquement disjoints sur B

2.b $\left(A_{i}\right)_{i \in I}$ est $\mathscr{L}_{1}$-disjointe si et seulement si les $A_{i}$ sont intégralement disjoints sur $B$.

3) Soit $E$ un $\varphi$-espace discret. Une famille $\left(x_{i}\right)$ de partie de $E$ est disjointe si et seulement si les $x_{i}$ sont sans élément commun deux à deux.

\subsection{2.- Propriétés élémentaires}

\section{0) Caractère fini}

Si $\left(X_{i}\right)$ est disjointe, toute $\left(Y_{i}\right)$ incluse dans $\left(X_{i}\right)$ est

disjointe Mieux :

$\left(x_{i}\right)_{i \in I}$ est disjointe si et seulement si $\forall J \in F(I),\left(x_{i}\right)_{i \in J}$ est disjointe et

$\left(x_{i}\right)_{i \in I}$ est disjointe si et seulement si $\forall\left(Y_{i}\right)$ telle que $\forall i \in I, \quad Y_{i} \in \mathscr{F}\left(x_{i}\right)$, $\left(Y_{i}\right)$ est disjointe.

et finalement 
$\left(x_{i}\right)_{i \in I}$ est disjointe si et seulement si $\forall J \in F(I)$ et $\forall\left(Y_{i}\right)$ telle que

$$
\begin{aligned}
\forall i \in I, \quad & Y_{i} \in \mathscr{F}\left(X_{i}\right), \\
& \left(Y_{i}\right)_{i \in J} \text { est disjointe. }
\end{aligned}
$$

\section{0) Cas triviaux}

Si pour tout $i$, sauf peut-être un, $x_{i} \subset \varphi(\phi)$

$\left(x_{i}\right)$ est trivialement disjointe.

Il est clair en outre que, pour qu'une famille $\left(x_{i}\right)_{i \in I}$ soit disjointe, il faut et il suffit que $\left(x_{i}\right)_{i \in J}$, où $J$ est l'ensemble des $i \in I$ tels que $x_{i} \not \varphi(\varnothing)$, soit disjointe.

Une famille $\left(x_{i}\right)_{i \in I}$ sera dite strictement disjointe si elle est dis jointe et si pour tout $i, x_{i} \notin \varphi(\varnothing)$.

\section{0). Associativité et commutativité.}

Soit $\left(x_{i}\right)_{i \in I}$ une famille de parties d'un $\mathscr{L}$-espace.

a) $\left(\mathrm{J}_{\ell}\right)_{\ell \in \mathrm{L}}$ étant une partition de $\mathrm{I},\left(\mathrm{x}_{i}\right)_{i \in \mathrm{I}}$ est disjointe si et seulement si $\forall \ell \in L,\left(x_{i}\right)_{i \in J_{\ell}}$ est disjointe et $\left(\mathrm{x}_{\mathrm{J}_{\ell}}\right)_{\ell \in \mathrm{L}} \quad$ est disjointe.

b) $\sigma$ étant une bijection de $I$ sur lui-meme, $\left(\mathrm{X}_{\mathbf{i}}\right)_{\mathbf{i} \in \mathrm{I}}$ est disjointe si et seulement si $\left(x_{\sigma(i)}\right)_{i \in I}$ est disjointe.

\subsection{3.- Liens avec la notion d'union directe}

Soit $\left(x_{i}\right)_{i \in I}$ une famille $\mathscr{L}$-disjointe de parties d'un $\mathscr{L}$-espace $(E, \mathscr{L})$. La condition (D) entraine que : $\forall \mathrm{Y} \in \mathscr{L}_{3}\left(\mathrm{X}_{\mathrm{I}}\right)$ telle que, $\forall \mathbf{i} \in \mathrm{I}, \quad \mathrm{Y} \cap \mathrm{X}_{\mathbf{i}} \in \mathscr{L}, \mathrm{Y} \in \mathscr{L}_{\mathrm{I}}$ et $\forall i \in I, \forall j \in I, i \neq j$, on a $x_{i} \cap x_{j} \subset \varphi(\phi)$. 
Donc $\left(X_{i}-\varphi(\varnothing)_{i \in I}\right.$ est une partition (au sens large)

de $x_{I}-\varphi(\phi)$ et :

$$
\left(x_{I}-\varphi(\phi), \mathscr{L} \text { induit }\right)=\underset{i \in I}{\uplus}\left(x_{i}-\varphi(\phi), \mathscr{L} \text { induit }\right)
$$

Il est immédiat de voir que cette condition suffit pour que $\left(x_{i}\right)$ soit $\mathscr{b}$-disjointe, et $l^{\prime}$ on déduit alors du théorème 4 de 2.3.3. la :

Proposition 1.- Soit $\left(x_{i}\right)_{i \in I}$ une famille de parties d'un $\mathscr{L}$-espace $E$ et pour tout $i \in I, \quad z_{i}=x_{i}-\varphi(\phi)$. Les propriétés suivantes sont équivalentes :

(D D) $\quad\left(x_{1}\right)$ est $\mathscr{L}$-disjointe

(D D $) \quad\left(z_{I}, \mathscr{L}_{\mid z_{I}}\right)=\biguplus \quad\left(z_{i}, \mathscr{L}_{\mid z_{i}}\right) \quad[i \in I]$

(D $D_{2}$ ) $\forall C \in l$ tel que Card $C \geqslant 2$ et $C \subset x_{I}$, il existe au moins un $i \in I$ tel que $c \subset x_{i}$, et $\forall i \in I, \forall j \in I-i, x_{i} \cap x_{j} \subset \varphi(\phi)$

(D $D_{3}$ ) $\forall c \in l$ tel que Card $c \geqslant 2$ et $c \subset x_{I}$, il existe un $i \in I$ et un seul tel que $c \cap x_{i} \neq \varnothing, \quad$ et $\forall i \in I, \forall j \in I-i, x_{i} \cap x_{j} \subset \varphi(\phi)$

$\left(D D_{4}\right) \quad \forall x \in F^{f}\left(x_{I}\right), r(x)=\sum r_{i}\left(x \cap x_{i}\right) \quad[i \in I]$

et alors, $\left.\forall i \in I, \quad x_{i} n_{\varphi\left(x_{I-i}\right.}\right) \subset \varphi(\phi)$

\subsection{4.- Dépendance entre $\mathscr{L}$ et la $\mathscr{L}$-disjonction}

Soient $\mathscr{C}$ et $\mathscr{C}^{\prime}$ deux structures de $\mathscr{L}$-espace sur un ensemble $E$. Si toute famille $\mathscr{b}$-disjointe est $\mathscr{C}^{\prime}$-disjointe, alors :

a) $\forall x \in \varphi(\phi),(x, x)$ est $\mathscr{b}$-disjointe, donc $\mathscr{b}^{\prime}$-disjointe : $x \in \varphi^{\prime}(\phi)$ donc :

$$
\varphi(\varnothing) \subset \varphi^{\prime}(\varnothing)
$$


b) $\forall c^{\prime} \in \mathscr{C}^{\prime}$ tel que Card $\mathrm{c}^{\prime} \geqslant 2, \quad\left(\mathrm{C}^{\prime}, \mathscr{\mathscr { C }} / \mathrm{C}^{\prime}\right)$ est un $\mathscr{L}$-espace 2-irréductible, sinon il existerait $\mathrm{C}^{\prime}{ }_{1}$ et $\mathrm{C}^{\prime}{ }_{2}$ tels que $\left(\mathrm{C}^{\prime}, \mathscr{C} / \mathrm{C}_{1}\right)=\mathrm{C}^{\prime}{ }_{1} \oplus \mathrm{C}_{2}{ }_{2}$, et $\left(C_{1}^{\prime}, C_{2}^{\prime}\right)$ serait $\mathscr{b}$-disjointe sans être $\mathscr{b}^{\prime}$-disjointe. Donc :

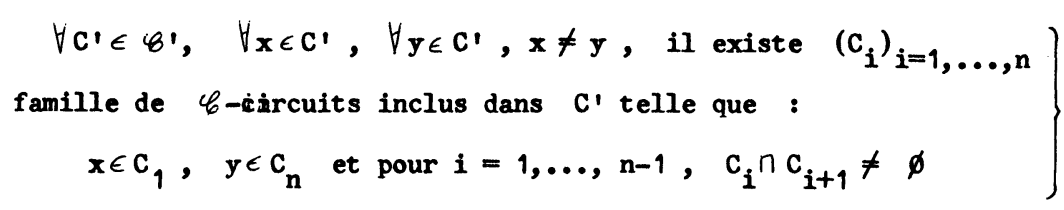

Il est aisé de voir que ces conditions suffisent. Donc :

Pour que toute famille $b$-disjointe soit $b^{\prime}$-disjointe, il faut et il suffit que $\mathscr{C}$ et $\mathscr{C}^{\prime}$ vérifient les propriétés (1) et (2) ci-dessus.

$$
\text { Si } \varphi(\phi)=\varphi^{\prime}(\phi),(2) \text { entraîne } \varphi^{\prime} \gg \nvdash, \text { la réciproque }
$$

étant fausse. En particulier une famille $\mathscr{L}\left(\right.$ resp. $\left.\mathscr{L}_{A}\right)$-disjointe n'est pas en général $\mathscr{L}_{A}($ resp. $\mathscr{L})$-disjointe, même dans les $\varphi$-espaces.

Si toute famille $\mathscr{b}$-disjointe est $\mathscr{C}^{\prime}$-disjointe et inversement,

alors $\mathscr{b}=\mathscr{b}$,

En effet, on aura alors $\varphi(\varnothing)=\varphi^{\prime}(\phi), \quad \mathscr{C}^{\prime} \gg \mathscr{C}$ et $\mathscr{C} \gg \mathscr{C}^{\prime}$.

\subsection{5.- La propriété de translation}

On peut construire des familles disjointes de proche en proche par accumulation en utilisant un procédé de "translation" de $\mathscr{L}$ : 
Proposition 2.- Soient E, F, G trois parties d'un $\mathscr{L}$-espace. Si E et G sont $\mathscr{L}$-disjoints, alors $\mathrm{E}$ et $\mathrm{F}$ sont $\mathscr{L}$-disjoints et $\mathrm{E}$ et $\mathrm{G}$ sont $\mathscr{L}_{\mathrm{F}}$-disjoints. La réciproque est vraie si $\mathscr{L}_{F} \ll \mathscr{L}$, ce qui est toujours le cas dans les $\varphi$-espaces.

Le début de l'énoncé se

vérifie aisément. Réciproquement, si $\mathrm{E}$ et $\mathrm{F}$

sont $\mathscr{L}$-disjoints et si $E$ et $G$ sont $\mathscr{L}_{F^{-}}$

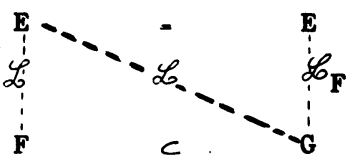
disjoints, alors :

a) $\mathbf{E} \cap \varphi(F) \subset \varphi(\varnothing)$ et $\mathbf{E} \cap \mathbf{F} \subset \varphi(F)$, donc $\mathbf{E} \cap \mathbf{F} \subset \varphi(\phi)$

et b) $\forall \mathrm{c} \in \boldsymbol{b}$ tel que Card $\mathrm{C} \geqslant 2$ et $\mathrm{C} \subset \mathbf{E} \cup \mathrm{G}$, on a :

$$
\text { CกENG } \quad \text { C } \operatorname{cn} \varphi(\phi)=\varnothing \quad: \quad \operatorname{CnE} \cap=\varnothing
$$

$$
\begin{array}{lll}
\text { Si } & \mathrm{C} \cap \mathrm{E}=\varnothing \quad, \quad \mathrm{C} \subset \mathrm{G} \\
\text { Si } & \mathrm{C} \cap \mathrm{E} \neq \varnothing \quad, \text { alors "en général" } \mathrm{C} \subset \mathrm{E} \cup \mathrm{F}, \text { donc } \mathrm{C} \subset \mathbf{E} .
\end{array}
$$

En effet, si $C \not E \cup F$ :

$$
\text { Quel que soit } \mathrm{C}^{\prime} \in \mathscr{C}_{F}, \quad \mathrm{C}^{\prime} \subset \mathrm{C} \text {, on a } \mathrm{C}^{\prime} \subset \mathrm{C} \text {. En effet : }
$$

- ou bien C' n'a qu'un élément $x^{\prime}$, alors $x^{\prime} \in \varphi(F)$ et $x^{\prime} \notin \varphi(\varnothing)$

(sinon $C^{\prime} \in \mathscr{C}$ et $C$ et $C^{\prime}$ seraient comparables). Donc $x^{\prime} \notin E$

- ou bien Card $C^{\prime} \geqslant 2$. Alors il existe $C^{\prime \prime} \in \mathscr{B}$ tel que $C^{\prime \prime}-F=C^{\prime}$. $C " \cap F \neq \varnothing$, sinon C et C" seraient comparables. Comme $E$ et G sont $\mathscr{L}_{F}$-disjoints, si $C^{\prime} \cap E \neq \varnothing, \quad C^{\prime} C E$, donc C"CEUF et on contredit le fait que $E$ et $F$ sont $\mathscr{L}$-disjoints; donc $C^{\prime} \cap E=\varnothing$ et $C^{\prime} \subset G$.

$$
\text { si } \mathscr{b}_{F} \ll \mathscr{b} \text {, tout } c \in \mathscr{C} \text { est réunion de } \mathscr{C}_{F} \text {-circuits, donc }
$$
C CG et alors on contredit C $\cap E \neq \varnothing$ et $\mathrm{C} \cap \mathrm{E} \cap \mathrm{G}=\varnothing$.

La condition la meilleure s'écrit :

$$
\forall \mathrm{C} \in \mathscr{C}, \quad \mathrm{C} \subset \mathrm{E} \cup \mathrm{G}, \quad \mathrm{C}-\mathrm{G} \text { n'est pas } \mathscr{C}_{F} \text {-libre }
$$


La proposition 2 est l'analogue pour la $\mathscr{b}$-disjonction de la proposition 7 de $[\mathrm{C}, \mathrm{II}, 5], \oint 2,3$, page 80 pour la disjonction linéaire (donc est vraie pour la disjonction algébrique).

En 3.2.4. sera énoncé un théorème général qui étend la proposition 2.

\section{2.- Les théorèmes fondamentaux sur la disjonction dans les $\varphi$-espaces.}

\subsection{1.- Lenme préliminaire sur les $\mathscr{L}$-espaces}

Lenme 1. $\left(\mathrm{Y}_{1}\right)_{i \in I}$ étant une famille de parties d'un $\mathscr{L}$-espace E, les propriétés suivantes sont équivalentes :

(D) $\quad Y_{I}$ est libre et les $Y_{1}$ sont disjoints deux à deux

$\left(D_{1}\right) \quad$ Pour tout $i \in I, Y_{i}$ est $\mathscr{L}_{0}\left[Y_{i}^{i}\right]$-libre où $Y_{i}^{\prime}=Y_{I-i}$

$\left(D_{2}\right) \quad$ Il existe un ordre total sur $I$ tel que, pour tout $i \in I \quad Y_{i}$ est $\mathscr{L}\left[\mathbf{Y}_{i}^{\prime \prime}\right]$-libre où $\mathbf{Y}_{i}^{\prime \prime}=U \mathbf{Y}_{j} \quad[j \in I$ et $\mathbf{j}<\mathbf{i}]$

$\left(D_{3}\right) \quad$ Pour tout ordre total sur $I$, pour tout $1 \in I, Y_{i}$ est $\mathscr{L}\left[Y_{i}^{\prime \prime}\right]$-libre.

En effet, d'après le corollaire 1 du théorème 2 de 2.1.3, ce lenme est vrai quand I a deux éléments, et (D) entraine $\left(D_{1}\right)$ et $\left(D_{3}\right)$.

Or $\left(D_{3}\right) \Longrightarrow\left(D_{2}\right)$ car tout ensemble peut être par exemple bien ordonné ; et $\left(D_{1}\right) \Longrightarrow\left(D_{3}\right)$ car, pour tout ordre total sur $I$, pour tout $i \in I, \quad \mathrm{Y}_{i}^{\prime \prime} \subset \mathrm{Y}_{i}^{\prime}$, donc $\mathscr{L}_{0}\left[\mathrm{Y}_{i}^{\prime}\right] \subset \mathscr{L}_{[}\left[\mathrm{Y}_{i}^{\prime \prime}\right] . \quad \mathrm{D}^{\prime}$ où $(D) \Rightarrow\left(D_{1}\right) \Rightarrow\left(D_{3}\right) \Longrightarrow\left(D_{2}\right)$. Il reste à démontrer : $\left(D_{2}\right) \Longrightarrow(D)$ : 
Ceci se démontre par récurrence sur Card I quand I est fini. Cette propriété étant vraie si Card $I=2$, supposons la vraie pour Card $I \leqslant n-1$, et soit $I=\{1, \ldots, n\}$ tel que :

$$
\forall \mathbf{i} \in \mathbf{I}, \quad \mathbf{Y}_{\mathbf{i}} \in \mathscr{L}_{\mathcal{L}}\left[\cup \mathbf{Y}_{\mathbf{j}} \quad[\mathbf{j} \in \mathbf{I}, \mathbf{j}<\mathbf{i}]\right]
$$

Alors $\left(Y_{i}\right)_{i \in I-n}$ vérifie $\left(D_{2}\right)$, donc (D) et par hypothèse de récurrence :

$$
Y=\bigcup_{i=1}^{n-1} Y_{i} \in \mathscr{L} \quad \text { et } \quad \forall i \in I-n \quad, \forall j \in I-n, i \neq j, Y_{i} \cap Y_{j} \varnothing
$$

En outre $Y_{n} \in \mathscr{L}[\mathbf{Y}]$, et comme $\mathbf{Y} \in \mathscr{L} ; \mathbf{Y}_{\mathbf{n}} \cup \mathrm{Y} \in \mathscr{L}$ et $\mathbf{Y}_{\mathbf{n}} \mathrm{H}^{\mathrm{Y}=\varnothing}$

Donc $\quad \bigcup_{i=1}^{n} Y_{i} \in \mathscr{b} \quad$ et $\quad \forall i \in I, \quad \forall j \in I \quad i \neq j \quad Y_{i} \cap Y_{j}=\varnothing$

Si I est infini et $s i\left(Y_{i}\right)$ vérifie $\left(D_{2}\right)$, pour tout $J \in \mathscr{F}(I)$, $\left(Y_{i}\right)_{i \in J}$ vérifie $\left(D_{2}\right)$ pour l'ordre total sur $J$ induit par celui de $I$; donc $\left(Y_{i}\right)_{i \in J}$ vérifie (D), et $\left(Y_{i}\right)_{i \in I}$ aussi en vertu du "caractère fini" de (D) vis-à-vis de I.

$$
\text { C Q F D }
$$

\subsection{2. - Applications aux $\varphi$-espaces}

Théorème 1.- Pour qu'une famille $\left(x_{i}\right)_{i \in I}$ de parties d'un $\varphi$-espace $E$ soit $\varphi$-disjointe, il faut et il suffit que pour tout $i$, il existe une $\varphi$-base $Y_{i}$ de $\varphi\left(X_{i}\right)$ telle que $\left(Y_{i}\right)_{i \in I}$ vérifie l'une des conditions équivalentes $(D),\left(D_{1}\right)$, $\left(D_{2}\right)$ ou $\left(D_{3}\right)$. Dans ce cas, toute famille $\left(X_{i}^{\prime}\right)$ incluse dans $\left(X_{i}\right)$ où les $X_{i}^{\prime}$ sont $\varphi$-libres possède ces propriétés.

$$
\text { Si }\left(x_{i}\right)_{i \in I} \text { est } \varphi \text {-disjointe, toute famille }\left(x_{i}^{\prime}\right) \text { incluse }
$$
dans $\left(X_{i}\right)$ où les $\left(X^{\prime}{ }_{i}\right)$ sont $\varphi$-libres vérifie (D) par définition, donc aussi $\left(D_{1}\right)$, $\left(D_{2}\right)$ et $\left(D_{3}\right)$; en particulier si pour tout $i, X^{\prime}{ }_{i}$ est une $\varphi$-base de $\varphi\left(X_{i}\right)$ incluse dans $\mathbf{x}_{\boldsymbol{i}}$. 
Réciproquement, s'il existe une famille $\left(Y_{i}\right)_{i \in I}$ vérifiant (D), $\left(D_{1}\right),\left(D_{2}\right)$ ou $\left(D_{3}\right)$ telle que pour tout $i \quad Y_{i}$ soit une $\varphi$-base de $\varphi\left(X_{i}\right)$, $\left(Y_{1}\right)$ vérifie $(D),\left(D_{1}\right),\left(D_{2}\right)$ et $\left(D_{3}\right)$ d'après le leme 1. Alors soit $\left(X_{1}\right)_{i \in I}$ une famille de parties $\varphi$-libres incluse dans $\left(x_{i}\right)$. Pour tout $i$, il existe $z_{i}$ $\varphi$-base de $\varphi\left(x_{i}\right), \quad x^{\prime}{ }_{i} \subset z_{i} \subset X_{i}$. Nous allons démontrer que $\left(z_{i}\right)$ vérifie $\left(D_{1}\right)$ :

$V_{i} \in I, Y_{i}$ est $\varphi\left[Y_{i}{ }_{i}\right]-l i b r e$ et $Y^{n_{i}}$ est $\varphi$-libre, donc $Y_{i}{ }_{i}$ est $\varphi \varphi_{Y} \Gamma^{-l i b r e .}$ Or pour tout $1, \varphi\left(Y_{i}\right)=\varphi\left(Z_{i}\right)=\varphi\left(X_{i}\right)$, donc $\varphi_{Y_{i}}=\varphi_{Z_{i}}$ :

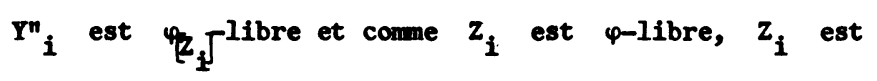
$\varphi_{Y}{ }_{1}$-libre.

Or pour tout $1, \varphi\left(Y_{i}\right)=\varphi\left(z_{i}\right)=\varphi\left(X_{1}\right)$, donc $\left.\varphi\left[Y_{1}\right]_{i}=\varphi_{i}^{\prime}{ }_{i}\right]$ et $z_{i}$ est $\left[{ }^{n} n_{1}\right]^{-l i b r e}:\left(z_{i}\right)$ vérifie $\left(D_{1}\right)$, donc $(D)$ et a fortiori $\left(X_{1}\right)$ vérifie (D).

Corollaire- Pour qu'une famille $\left(x_{i}\right)_{i \in I}$ soit $\varphi$-disjointe il faut et il suffit que l'une des conditions suivantes soit réalisée :

$$
\left(D{ }_{0}\right) \quad\left(\varphi\left(x_{i}\right)\right)_{i \in I} \text { est } \varphi \text {-disjointe }
$$

(D' $\left.{ }_{1}\right)$ Pour tout $i \in I$, sauf peut-etre un, il existe une $\varphi$-base de $\varphi\left(x_{i}\right)$ qui est $\varphi\left[\mathrm{X}_{1}\right]$-libre.

$\left(D^{\prime}{ }_{2}\right)$ Il existe un ordre total sur I tel que, pour tout $i \in I$, il existe une $\varphi$-base de $\varphi\left(x_{1}\right)$ qui est $\varphi\left[X_{1}\right]$-libre.

On obtient toutes les familles strictement $p$-disjointes ainsi : Soit $I$ une partie $\varphi$-libre d'un $\varphi$-espace $E$ et $\left(x_{i}\right)_{i \in I}$ une partition (au sens strict) de $X$. Alors $\left(X_{i}\right)$ est strictement $\varphi$-disjointe et toute famille incluse dans $\left(\varphi\left(x_{i}\right)\right)_{i \in I}$ est $\varphi$-disjointe. 


\subsection{3.- La propriété de translation dans les $\varphi$-espaces.}

Théorème 2 (La propriété de translation)

$$
\text { Soit }\left(x_{i}\right)_{i \in I} \text { une famille de parties d'un } \varphi \text {-espace } E \text { et }
$$

pour tout $i \in I, \quad\left(x_{i}^{j}\right)_{j \in J_{i}}$ une famille bien ordonnée de parties de $x_{i}$ telles que : $U x_{i}^{j} \quad\left[j \in J_{i}\right]=x_{i}$

( $\mathrm{J}_{i}$ bien ordonné et $\left(\mathrm{x}_{i}^{j}\right)$ croissante). Alors les propriétés suivantes sont équivalentes :

1) $\quad\left(x_{i}\right)_{i \in I}$ est $\varphi$-disjointe

2) (*) $\forall\left(j_{i}\right) \in \prod_{i \in I}^{d} j_{i}, \quad\left(x_{i}^{j_{i}}\right)_{i \in I}$ est $\left.b \underset{i \in I}{\bigcup} \bigcup_{\substack{k \in J_{i} \\ k<j_{i}}} x_{i}^{k}\right]$-disjointe

démonstration :

1) $\Longrightarrow$ 2) :

$$
\text { En effet, pour tout }\left(j_{i}\right) \in \prod_{i \in I} J_{i} \text {, soit : }
$$

$A=\bigcup_{i \in I} \cup x_{i}^{k} \quad\left[k \in J_{i} \quad\right.$ et $\left.\quad k<j_{i}\right] ;$ et $x=\bigcup_{i \in I} x_{i}^{j_{i}}$

Vérifions $\left(D_{3} D_{3}\right.$ pour $\left(x_{i}^{j_{i}}\right)_{i \in I}$ et $\mathscr{L}_{[A]}$ :

(*) $\prod_{i \in I}^{d} J_{i}=\left\{\left(j_{i}\right) \mid\left(j_{i}\right) \in \prod_{i \in I} J_{i}\right.$ et $j_{i}$ est le premier élément de $\left.J_{i}\right\}$ pour tout $i \in I$, sauf un nombre fini

On sait qu'alors, si $I$ est bien ordonné, $\prod_{i \in I}^{d} J_{i}$ est bien ordonné en considérant l'ordre lexicographique obtenu en prenant pour ordre total sur I l'ordre inverse du bon ordre donné (voir [21], chapitre II, 4.3, théorème 14, p. 65). 


$$
\forall c \in G[A] \text { tel que Card } c \geqslant 2 \text { et } c c x, \exists c^{\prime} \in l, c^{\prime}-A=c \text {. }
$$
Comme $A C X, C^{\prime} \subset X$ et Card $C^{\prime} \geqslant 2$, donc d'après $\left(D_{3}\right)$ pour $\left(X_{1}\right)$, il existe un $i \in I$ et un seul tel que $\operatorname{cin} x_{i} \neq \emptyset$; et comme $C C^{\prime}$, il en est de meme fortiori pour $c$.

2) $\longrightarrow$ 1)

Si on considère un bon ordre sur $I, J=\Pi^{d} J_{i} \quad[i \in I] \quad$ est binn ordonné $(*)$ et l'on demonteer par récurrence traufinie $\left(*^{*}\right)$ sur ord $J$ que, pour tout $\left.\left.\varphi \in \oint_{m}(E), 2\right) \Longrightarrow 1\right)$.

La propriété est triviale si Ord $\mathrm{J}=0$ ou 1. A supposer que l'on ait démontré que :

$\forall \varphi \in \Phi_{m}(E), \forall\left(x_{1}^{j}\right)$ telle que ord $J<\lambda,\left(x_{1}^{j}\right)$ vérifie 2$) \Rightarrow\left(x_{1}\right)$ vérifie 1), soit alors $\left(x_{i}^{j}\right)$ telle que ord $J=\lambda$ et vérifiant 2$)$ dans $(E, \varphi)$.

Si I n'a pas de plus grand elément, i.e. si $\lambda$ n'a pas de prédécesseur, pour tout $\left(j_{i}\right) \in J,\left(x_{i}^{j_{i}}\right)_{i \in I}$ est $\mathscr{L}$-disjointe :

En effet soit pour tout $i \in I, \quad J_{1}^{\prime}=\left[\leftarrow, J_{1}\right]$ dans $J_{i}$ $J^{\prime}=\prod^{d} J_{i}[i \in I]$ est fini, donc ord $J^{\prime}<\lambda$ et comme $\left(\left(x_{i}^{j}\right) \quad\left[J_{0} \in J^{\prime}\right]\right)[i \in I]$ vérifie 2), a fortiori il vérifie 1):

$$
\left(x_{i}^{j_{1}}\right)_{i \in I} \quad \text { est } \mathscr{L}_{- \text {-disjointe. }}
$$

Donc, en vertu du caract ìre fini de la disjonction, $\left(x_{i}\right)_{i \in I}$ est $\mathscr{L}$-disjointe.

$\left({ }^{* *}\right)$ En ne considérant, pour un ensemble $E$ firé, que les familles $\left(x_{1}^{j}\right)$ telles que : $\forall i \in I, \quad\left(x_{i}^{j}\right)$ soit strictement croissante et $\forall i \in I, \forall i \in I-i$, $x_{i} \cap x_{i}, \subset \varphi(\phi)$, tous les ord $J$ possibles forment un ensemble. Soit Sup Ord $J=\alpha$; la récurrence transfinie se fait dans $a$. 
Si I a un plus grand élément, i.e. si $\lambda$ a un prédécesseur, pour tout $i \in I$, $\mathrm{J}_{\mathbf{i}}$ a un plus grand élément, soit :

$$
\operatorname{Max} J_{i}=\beta_{i} \text { et Min } J_{i}=\alpha_{i}
$$

La borne supérieure des éléments de $\prod^{d} J_{i}$ dans $\prod J_{i}$ existe et est $\left(\beta_{i}\right)$, donc pour presque tout $i \in I$, sauf un nombre fini, on a $\beta_{i}=\alpha_{i}$, i.e. $J_{i} n^{\prime} a$ qu'un élément. Soit :

$\left\{i_{1}, \ldots, i_{n}\right\} \quad I^{\prime e n s e m b l e ~ d e s ~} i \in I$ tels que Card $J_{i} \geqslant 2$, arec

$$
i_{1}<\ldots<i_{n}
$$

- Soit pour $i \in I-I_{1}, J^{\prime}=J_{i}$ et $Y_{i}=\mathbf{x}_{i}$

et pour $i=i_{1}, \quad J_{i_{1}}=J_{i_{1}}-\beta_{i_{1}}$ et $Y_{i_{1}}=U \mathbf{I}_{i_{1}}\left[j \in J_{i_{1}}\right.$ et $\left.j<\beta_{i_{1}}\right]$, et $\forall \mathbf{i} \in \mathbf{I}, \forall \mathbf{j} \in \mathrm{J}^{\prime}, \quad, \quad \mathbf{Y}_{\mathbf{i}}^{\mathbf{j}}=\mathbf{x}_{\mathbf{i}}^{\mathbf{j}}$

$\left(X_{i}^{j}\right)$ vérifiant 2$),\left(Y_{i}^{j}\right)$ vérifie 2) aussi, et comme

$$
\begin{aligned}
& \operatorname{Ord}\left(\pi^{d} J_{i}^{\prime}\right)<\operatorname{Ord} J=\lambda \\
& \left(Y_{i}\right)_{i \in I} \text { est } \varphi \text {-disjointe }
\end{aligned}
$$

- Soit pour $i \in I-i_{1}, J^{n_{1}}=J_{i}$, pour $i=i_{1}, J^{n_{i}}=\left\{\beta_{i_{1}}\right\}$, et $\forall i \in I \quad, z_{i}=x_{i}$ et $\forall j \in J^{\prime \prime}, \quad z_{i}^{j}=x_{i}^{j}$

$\left(x_{i}^{j}\right)$ vérifiant 2$)$ dans $(E, \varphi),\left(z_{i}^{j}\right)$ vérifie 2) dans $\left(E, \varphi\left[Y_{1}\right]\right)$. Or ord $\left(\pi^{d} J_{i}\right)<$ ord $J=\lambda \quad$ donc

$$
\left(x_{i}\right)_{i \in I} \text { est } \varphi\left[x_{i_{1}}\right] \text {-disjointe }
$$

- D'après l'associativité de la disjonction, (1) et (2) entrainent :

$$
\begin{array}{ccc}
\left(x_{i}\right) & {\left[i \in I-i_{1}\right]} & \varphi \text {-disjointe } \\
x_{I}-i_{1} & \text { et } \quad Y_{i_{1}} & \text { solt } \varphi \text {-disjoints } \\
x_{I}-i_{1} & \text { et } \quad Y_{i_{1}} & \text { sont } \varphi\left[x_{i_{1}}\right] \text {-disjoints }
\end{array}
$$

donc d'apies la proposition 2 de 3.1 .5 , 


$$
x_{I-1} \text { et } x_{i_{1}} \text { sont } \varphi \text {-disjoints }
$$

donc (associativité de $(3)$ et $(6)), \quad\left(x_{i}\right)_{1 \in I}$ est $p$-disjointe

$$
\text { C.Q.F.D. }
$$

Remarque 1) Les conditions : " $\forall\left(j_{i}\right) \in \Pi^{d} J_{i} n$ sont indépendantes en ce sens que, pour tout $\left(j_{i}\right) \in \pi^{d} J_{i}, i_{k}$ existe des familles $\left(x_{i}^{j}\right)$ telles que, $\forall\left(k_{i}\right) \in \pi^{d} j_{i}, \quad\left(k_{i}\right) \neq\left(j_{i}\right), \quad\left(x_{i}\right)_{i \in I} \quad$ est $\mathscr{L}[]$-disjointe et

$$
\begin{gathered}
\left(x_{i}^{j}\right)_{i \in I} \quad n^{\prime} \text { est pas } \mathscr{L}[]_{\text {-disjointe alors que }\left(x_{i}\right)_{i \in I}} \text { ''est pas } \\
\mathscr{L}_{\text {-disjointe. }}
\end{gathered}
$$

Remarque 2) Les conditions de bons ordres sur les $J_{i}$ sont absolument nécessaires. Il eat en effet aisé de construire des contre-exemples ( $\varphi$-espace) quand un ensemble $J_{i}$ 'est plus bien ordonné.

L'intérêt de cette démonstration est qu'elle s'étend aux Ĺb-espaces grâce à la proposition 2. D'où le :

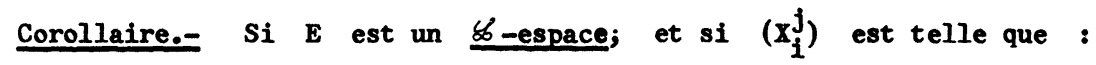

$$
\begin{gathered}
\forall\left(j_{i}\right) \in \prod_{i}^{d} J_{i}, \forall\left(k_{i}\right) \in \prod_{i}^{d} J_{i} \text { tels que } \forall i \in I, j_{i} \leq k_{i}, \\
\left.\mathscr{L}\left[\bigcup_{i \in I} \bigcup_{\substack{k \in J_{i} \\
k<k_{i}}}^{x_{i}^{k}}\right] \ll \mathscr{L}_{i \in I} \bigcup_{\substack{k \in J_{i} \\
k<j_{i}}} x_{i}^{k}\right]
\end{gathered}
$$

Alors les propriétés 1) et 2) sont équivalentes pour $\left(x_{i}^{j}\right)$ 
3.2.4.- Application a la construction des bases et au calcul du rang dans un p-espace

Soit $\left(X_{i}\right)_{i \in I}$ une famille de parties d'un $\varphi$-espace et $\leqslant$ un ordre total sur I. On appelle segment de I toute partie $S$ de I telle que, $\forall i \in S, \forall j \in I$ tels que $j<i$, on a $j \in S$. L'on note $\underline{\varphi(I) \text { l'ensemble }}$ des segments de I sauf I lui-meme. Alors on a le :

\section{Théorène 3.-}

Soit $\left(x_{i}\right)_{i \in I}$ une famille de parties d'un $\varphi$-espace $E$ et $\leqslant$ un ordre total sur I. Soit pour tout $S \in \varphi(I)$,

$$
x^{s}=\bigcap_{i \in I-S} \varphi\left(x_{[\leftarrow, i]}\right) \text { et } \quad y_{S} \text { une } r\left[x_{S}\right] \text {-base de } x^{s} \text {. }
$$

Alors les
et on $\mathrm{Y}_{S}$ sont disjointes deux à deux et $\mathrm{Y}_{\varphi(I)}$ est une r-base de $\varphi\left(X_{I}\right)$

$$
\mathbf{r}\left(\mathbf{x}_{\mathbf{I}}\right)=\sum \mathbf{r}\left[\mathbf{x}_{\mathbf{S}}\right]\left(\mathbf{x}^{\mathbf{s}}\right) \quad[\mathbf{s} \in \mathscr{\mathcal { L }}(\mathbf{I})]
$$

\section{Demonstration :}

$\varphi(I)$ est totalement ordonné et il est immédiat de vérifier la propriété $\left(D_{2}\right)$ du lemme 1 pour $\left(\mathrm{Y}_{S}\right)_{S \in \mathcal{Y}}(\mathrm{I})$ où $\mathscr{Y}(\mathrm{I})$ est muni de l'ordre total ci-dessus. Donc les $Y_{S}$ sont disjoints deux à deux et $Y=Y_{\varphi(I)}$ est libre.

$$
\begin{aligned}
& \text { Comme } Y \subset \varphi\left(X_{I}\right), \varphi(Y)<\varphi\left(X_{I}\right) \text { - Réciproquement : } \\
& \forall x \in \varphi\left(x_{I}\right) \text {, soit } s_{0}=\left\{i \mid i \in I \text { et } x \notin \varphi\left(X_{[\leftarrow, i]}\right)\right\} \in \varphi(I) \\
& S_{0} \varsubsetneqq I \quad \text { et } \quad x \in \varphi\left(Y S_{S_{0}} \cup X_{S_{0}}\right) \quad, x \notin \varphi\left(X_{S_{0}}\right) \\
& \text { Si } x \in \varphi\left(\mathrm{Y}_{\mathrm{S}_{0}}\right) \quad, \quad x \in \varphi(\mathrm{Y}) ; \text { Sinon } x \notin \varphi\left(\mathrm{Y}_{\mathrm{S}_{0}}\right) \\
& \text { donc } S_{1}=\left\{i \mid i \in S_{0} \quad \text { et } x \notin \varphi\left(Y_{S_{0}} \cup x_{[\leftarrow, i\}}\right) \in \mathcal{Y}(I)\right.
\end{aligned}
$$




$$
\begin{array}{cll}
\mathrm{s}_{1} \risingdotseq \mathrm{s}_{0} \quad \text { et } \quad & x \in \varphi \quad\left(\mathrm{y}_{\mathrm{S}_{0}} \cup \mathrm{y}_{\mathrm{S}_{1}} \cup \mathrm{x}_{\mathrm{S}_{1}}\right) \\
& x \notin \varphi \quad\left(\mathrm{y}_{\mathrm{S}_{0}} \cup \mathrm{x}_{\mathrm{S}_{1}}\right)
\end{array}
$$

Si $x \in \varphi\left(Y_{S_{0}} \cup Y_{S_{1}}\right), x \in \varphi(Y) ;$ Sinon $x \notin \varphi\left(Y_{S_{0}} \cup Y_{S_{1}}\right)$

On construit ainsi de proche en proche une suite strictement

décroissante

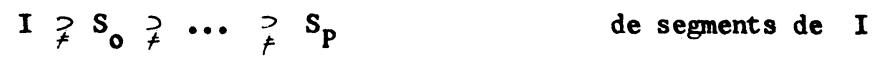

tels que

$$
x \in \varphi \quad\left(Y_{S_{0}} \cup Y_{S_{1}} \cup \ldots \cup \mathbf{Y}_{S_{P}} \cup X_{S_{P}}\right)
$$

mais

$$
x \notin \varphi \quad\left(Y_{S_{0}} \cup \ldots \cup Y_{S_{i-1}} \cup Y_{S_{i+1}} \cup \ldots \cup Y_{S_{P}} \cup x_{S_{P}}\right) \text { pour } i=1, \ldots, P
$$

Si $x \in \varphi \quad\left(\mathrm{Y}_{\mathrm{S}_{0}} \cup \ldots \cup \mathrm{Y}_{\mathrm{S}_{\mathrm{P}}}\right) \quad, \quad \mathrm{x} \in \varphi(\mathrm{Y})$. Sinon, on considère

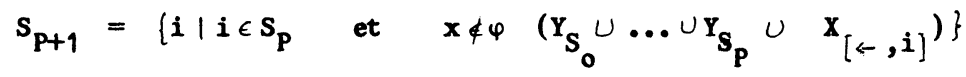

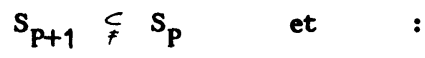

pour $i=1, \ldots, P+1 \quad, \quad x \notin \varphi\left((\underbrace{P+1}_{\substack{j=1 \\ j \neq i}} Y_{S_{j}}) \cup x_{S_{P+1}}\right.$

$$
\text { Si } x \notin \varphi\left(\bigcup_{j=1}^{P+1} Y_{S_{j}}\right), \text { l'on peut itérer. }
$$

Or, on ne peut itérer indéfiniment ce processus, car sinon on aurait une suite $\left(S_{P}\right)_{P \in N}$ de segments strictement décroissante telle que $\begin{array}{ll}\text { si } S_{\omega}=\bigcap_{P \in N} S_{P} \quad \text { et } & \psi=\varphi\left[X_{S_{\omega}}\right] \\ \left(Y_{\left.S_{P}\right)_{P} \in N} \text { est } \psi \text {-dis jointe et }\right. & Y^{\prime}=U Y_{S_{P}}[P \in N] \text { est } \psi \text {-libre }\end{array}$ $\mathbf{X} \in \psi\left(\mathrm{Y}^{\prime}\right) \quad$, mais pour tout $\mathrm{P} \in \mathrm{N}, \quad \mathbf{X} \notin \psi\left(\mathrm{Y}^{\prime}-\mathrm{Y}_{\mathrm{S}_{\mathbf{P}}}\right)$. 
Soit $Y^{\prime} x+x$ le $\psi$-circuit unique inclus dans $Y^{\prime}+x \cdot Y^{\prime} x$ ne peut rencontrer qu'un nombre fini de $Y_{S_{P}}$, ce qui contredit le fait que $x \in \psi\left(Y^{\prime}-Y_{S_{P}}\right)$ pour une infinité de $\mathrm{Y}_{\mathrm{S}_{\mathrm{P}}}$.

$$
\begin{aligned}
& \text { D'où un entier } p \text { tel que } x \in \varphi\left(\bigcup_{j=1}^{p} y_{S_{j}}\right): x \in \varphi(Y) \\
& Y \text { est donc une base de } \varphi\left(x_{I}\right)
\end{aligned}
$$

En particulier quand I est bien ordonne, tous les segments différents de $I$ oont de la forme $] \leftarrow, i[$ où $i \in I$

$$
\text { Si } s=j \leftarrow, i\left[=s_{i} \quad, \quad x^{S_{i}}=\varphi\left(x_{[\leftarrow, i]}\right) \cdot\right. \text { On a alors }
$$

le résultat plus précís suivant :

\section{Corollaire}

Soit $\left(x_{i}\right)_{i \in I}$ une famille de parties d'un $\varphi$-espace $E$ et un bon ordre sur I. Si pour tout $i \in I, Y_{i}$ est une $\varphi\left[X_{[\leftarrow, i[]-b a s e ~ d e}\right.$ $x_{i}$, alors les $Y_{i}$ sont des parties disjointes deux à deux et $Y_{I}$ est une $\varphi$-base de $x_{I}$, et on a :

$$
r\left(x_{I}\right)=\sum_{i \in I} r\left[x_{] \leftarrow, i[}\right] \quad\left(x_{i}\right)
$$

En particulier, si A et B sont deux parties de E, on a :

$$
r(A \cup B)=r(A)+r[A](B)=r(B)+r(B](A)
$$


3.3.- Caractérisation des familles disjointes avec le rang ( $\varphi$-espaces)

3.3.1.- Caractérisation par les sommes.

Théorème 4.-

Soit $\left(\mathrm{X}_{i}\right)_{i \in I}$ une famille de parties d'un $\mathscr{L}$-espace $(E, \mathscr{E})$,

on a :

$$
r\left(x_{I}\right) \leq \sum_{i \in I} r\left(x_{i}\right)
$$

Si $\left(x_{i}\right)$ est $\mathscr{L}$-disjointe, on a l'égalité.

Réciproquement, si $(E, \mathscr{L})$ est un $\varphi$-espace, si $r\left(x_{i}\right)$ est fini pour tout $i \in I$ et $s^{\prime} i l$ existe une famille $\left(J_{\ell}\right)_{\ell \in L}$ de parties finies de $I$, cofinale à $f(I)$ et telle que, pour tout $l \in L$,

$$
r\left(x_{J_{l}}\right)=\sum r\left(x_{i}\right) \quad\left[i \in J_{l}\right]
$$

Alors $\left(x_{i}\right)$ est $\varphi$-disjointe.

Démonstration

Si $Y$ est une base de $x_{I}$ telle que $r\left(X_{I}\right)=$ Card $Y$ et si on considère un bon ordre sur $I$, on définit par récurrence transfinie sur $i \in I$, $Y_{i}=Y \cap X_{i}-Y_{[\hookleftarrow, i[} \cdot$ Alors $Y_{I}=Y$, les $Y_{i}$ sont disjoints deux à deux et :

$$
r\left(x_{I}\right)=\operatorname{Card} Y=\sum \operatorname{Card} y_{i} \quad[i \in I] \leq \sum r\left(x_{i}\right) \quad[i \in I] .
$$

D'où (II). Si $\left(X_{i}\right)$ est $\mathscr{L}$-disjointe, soit pour tout $i \in I \quad Y_{i}$ une base de $x_{i}$ telle que $r\left(x_{i}\right)=\operatorname{Card} Y_{i}, \quad Y_{I} \in \mathscr{L}$ et les $Y_{i}$ sont disjoints deux à deux. Donc :

$$
r\left(x_{I}\right) \geqslant \operatorname{Card} y_{I}=\sum r\left(x_{1}\right) \quad[i \in I]
$$


et l'égalité.

La réciproque résulte du fait que la disjonction est de caractère

fini et du :

Corollaire - Soit $\left(X_{i}\right)_{i \in I}$ une famille finie de parties d'un $\varphi$-espace telle que pour tout $i \in I, r\left(X_{i}\right)$ soit finie. Alors les propriétés suivantes sont équivalentes :
a) $\left(x_{i}\right)$ est $\varphi$-disjointe
b) $\quad r\left(x_{I}\right)=\sum r\left(x_{i}\right) \quad[i \in I]$

L'on a démontré $a) \Rightarrow b$ ). Réciproquement, soit pour tout $i \in I$

$Y_{i}$ une base de $x_{i}$. Alors $Y_{I}$ engendre $x_{I}$ et d'après $\left.b\right)$ :

$$
r\left(X_{I}\right)=\operatorname{Card} Y_{I}=\sum \operatorname{Card} Y_{i} \quad[i \in I] \text {. }
$$

Les conditions de finitude font que $\left(Y_{i}\right)$ vérifie $(D)$, donc, d'après le théorème $1,\left(x_{i}\right)$ est disjointe.

Remarque : Si l'une des conditions de finitude exigée pour la réciproque n'est pas vérifiée, ou si $(E, \mathscr{Z})$ n'est pas un $\varphi$-espace, la réciproque n'est plus vraie.

\subsection{2. - Caractérisation par translation de $\varphi$}

Théorème 5.- Soit $\left(x_{i}\right)_{i \in I}$ une famille de parties d'un $\mathscr{L}$-espace $(E, \mathscr{L})$.

a) $\forall \mathbf{i} \in \mathbf{I}, \quad r\left(\mathbf{x}_{\mathbf{i}}\right) \geqslant \mathbf{r}\left[\mathrm{x}_{\mathrm{I}-\mathbf{i}}\right]\left(\mathrm{x}_{\mathbf{i}}\right)$

Si $\left(x_{i}\right)$ est $\mathscr{L}$-disjointe, on a l'égalité dans (III). 
Réciproquement, si pour tout $i \in I$, sauf peut-être un, $r\left(x_{1}\right)$ est fini et l'égalité est vérifiée dans (III), et si $(E, \mathscr{L})$ est un $\varphi$-espace, alors $\left(x_{1}\right)$ est $\varphi$-disjointe.

b) Pour tout ordre total sur I,

$$
\forall i \in I, r\left(x_{i}\right) \geqslant r\left[x_{[-, i]}\right] \quad\left(x_{i}\right)
$$

$$
\text { Si ( } \left.x_{1}\right) \text { est } \mathscr{L} \text {-disjointe, on a l'égalité dans (IV). }
$$

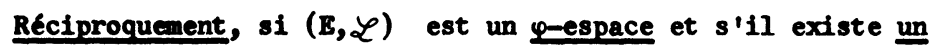
ordre total sur I tel que, pour tout $I \in I$, sauf peut-être le plus petit, $r\left(X_{i}\right)$ soit fini et on ait l'égalité dans (IV), alors $\left(x_{i}\right)$ est $\varphi$-disjointe.

\section{Demonstration}

Les inégalités (III) et (IV) résultent de ce que, pour tout A, $\mathbf{r}[\mathbf{A}] \leq \mathbf{r}$.

Si $\left(x_{i}\right)$ est $\mathscr{L}$-disjointe, pour tout $i \in I, x_{i}$ et $x_{I-i}$ sont $\mathscr{L}$-disjoints, et, utilisant la propriété ( $\left(\mathrm{D}_{3}\right)$ par exemple, toute partie $\mathscr{L}$-libre de $x_{i}$ est $\mathscr{L}\left[x_{I-1}\right]$-libre. Donc

$$
r\left(x_{i}\right)=r\left[x_{I-i}\right]\left(x_{i}\right)
$$

let a fortiori $r\left(x_{i}\right)=r\left[x_{[\leftarrow, i[}\right]\left(x_{i}\right) \quad$ pour tout ordre total fixé a pptori sur $I$.

$$
\text { Les réciproques résultent du corollaire du théorème 1, }
$$$$
\left.\left(D{ }_{1}\right) \rightarrow\left(D^{\prime}{ }_{0}\right) \quad \text { (resp. }\left(D^{\prime}{ }_{2}\right) \rightarrow D{ }_{0}\right) \text {. En effet, pour tout } i \in I \text {, soit }
$$
$Y_{i}$ une $r\left[X_{I-i}\right]$-base (resp. $r\left[x_{[\leftarrow, i[}\right]$-base) de $x_{i} \cdot Y_{i}$ est r-libre et fini, et comme $r\left(x_{i}\right)=\operatorname{Card} Y_{i}, Y_{i}$ est une $r$-base de $x_{i}$. 
Remarque : La réciproque n'est plus vraie si l'une des conditions de finitude exigées n'est pas vérifiée, ou si $(E, \mathscr{L})$ n'est pas un $\varphi$-espace.

Voici en particulier un énoncé qui entraine la proposition 10, p. 103, de Bourbaki $[$ C, II, 5], $\$ 5.4$, :

Corollaire Soient A et B deux parties d'un $\mathscr{L}$-espace $(E, \mathscr{L})$. L'on a :

$$
\begin{aligned}
& r(A \cup B) \leq r(A)+r(B) \\
& r[A](B) \leq r(B) \\
& r[B](A) \leq r(B)
\end{aligned}
$$

Si A et B sont $\mathscr{L}$-disjoints, on a l'égalité dans chacune de ces relations.

$$
\text { Réciproquement, si }(E, \mathscr{L}) \text { est un } \varphi \text {-espace : }
$$

a) Si l'égalité est vérifiée dans la relation (1) et si l'un des deux membres est fini, A et B sont $\varphi$-disjoints.

b) Si l'égalité est vérifiée dans la relation (2) et si $r(B)$ est fini, A et B sont $\varphi$-disjoints. 


\section{4.- La disjonction dans une L-algèbre}

\subsection{1.- Généralités}

On appelle $\mathscr{L}$-algèbre la donnée sur une structure de $\mathscr{L}$-espace $(E, \mathscr{L})$ d'une loi de composition interne $(x, y) \rightarrow x . y$ notée multiplicativement et telle que :

$$
\forall \in \in \mathscr{C}, \forall x \in E, \quad x . c \text { et c.x sont réunions de } \mathscr{b} \text {-circuits }
$$

Alors on a : $\varphi(\varnothing) . E=E . \varphi(\varnothing) \subset \varphi(\varnothing)$

\section{Définition 2.-}

Soit $(E, \mathscr{L},$.$) une \mathscr{L}$-algèbre et $\left(x_{i}\right)_{i \in I}$ une famille de parties de $E$. $\left(x_{i}\right)$ est dite $\mathscr{L}$-.-disjointe (ou simplement 2-disjointe) si elle vérifie les propriétés suivantes :

a) $\forall\{i, j\} \in \mathfrak{F}_{2}(I), \forall x \in \mathbf{x}_{\mathbf{i}^{-\varphi}}(\phi), \forall \mathbf{y} \in \mathbf{x}_{\mathbf{j}}-\varphi(\phi), \mathbf{x}_{\mathbf{0}} \mathbf{y}=\mathbf{y} \cdot \mathbf{x}$

b) $\forall\{i, j, k\} \in \mathscr{F}_{3}(I), \forall x \in \mathbf{x}_{\mathbf{i}}-\varphi(\phi), \forall \mathbf{y} \in \mathbf{x}_{\mathbf{j}}-\varphi(\phi), \forall z \in \mathbf{x}_{k}-\varphi(\varnothing)$,

$$
x\left(\begin{array}{ll}
y & z
\end{array}\right)=\left(\begin{array}{ll}
x & y
\end{array}\right) z
$$

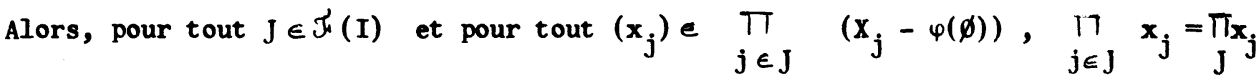
ne dépend pas de la manière dont on le calcule (ordres et parenthèses).

c) Pour toute famille $\left(\mathrm{Y}_{\mathbf{i}}\right) \subset\left(\mathrm{x}_{\mathbf{i}}\right)$ telle que pour tout $\mathbf{i}, \mathrm{Y}_{\mathbf{i}} \in \mathcal{L}$,

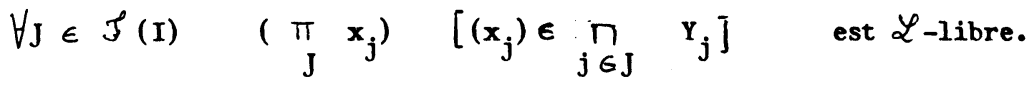

exemple. Soit A une algèbre, $B$ une sous-algèbre de $A$, pour tout $X \in E, \varphi(X)$ le sous-B-module engendré par $X$ et $x . y$ la multiplication dans $A \cdot\left(A, \mathscr{L}_{\varphi},.\right)$

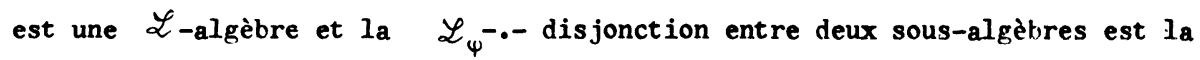
disjonction linéaire sur B entre ces sous-algèbres. 
Propriétés élémentaires.

10) Caractère fini : mêmes équivalences qu'en 3.1.2. 10)

$\left.2^{\circ}\right)$ Cas triviaux : Si $x_{i} \subset \varphi(\varnothing)$ pour tout $i \in I$, sauf peut-être un, $\left(x_{i}\right)$ est trivialement 2-dis jointe.

$\left.3^{\circ}\right)$ Commutativité : $\left(x_{i}\right)_{i \in I}$ est 2-disjointe si et seulement si $\left(x_{\sigma(i)}\right)_{i \in I}$ est 2-disjointe quand $\sigma$ est une bijection.

$\left.4^{\circ}\right)$ Si $\left(x_{i}\right)$ est 2-disjointe, pour tout $\{i, j\} \in \mathcal{F}_{2}(I), \quad r\left(x_{i} \cap x_{j}\right) \leq 1$

Proposition 3.- (Associativité de la 2-disjonction).

Soit $\left(x_{i}\right)_{i \in I}$ une famille de parties d'une $\mathscr{L}$-algèbre $(E, \mathscr{L},$.

et $\left(\mathrm{J}_{\ell}\right)_{\ell \in \mathrm{L}}$ une partition de $\mathrm{I}$. Alors considérons les propriétés suivantes :

(1) $\quad\left(x_{i}\right)_{i \in I}$ est 2-disjointe

(2) $\forall l \in \mathrm{L},\left(\mathrm{x}_{\mathrm{i}}\right)_{i \in \mathrm{J} l}$ est 2-disjointe

et (*) $\left(\pi x_{i}\left[i \in J_{\ell}\right)_{l \in L}\right.$ est 2-disjointe

L'on a toujours $(1) \Longrightarrow(2)$. La réciproque $(2) \Rightarrow(1)$ est vraie quand : $\forall(i, j) \in \mathcal{F}_{2}(I), \forall k \in I-\{i, j\}, \forall x \in X_{i}-\varphi(\varnothing), \forall y \in X_{j}-\varphi(\phi), \forall z \in X_{\ell}-\varphi(\varnothing)$ $x(y z)=\left(\begin{array}{ll}x & y\end{array}\right) z$

ce qui est toujours vérifié quand - est associative.

(*) $\pi x_{i}[i \in I]=\left\{\underset{J}{\pi} \quad x_{j} \mid J \in \mathcal{f}(I), J \neq \varnothing\right.$ et $\left.\left(x_{j}\right) \in \prod_{j \in J} x_{j}\right\}$ 
3.4.2.- La disjonction dans une $\varphi$-algèbre

Définition $3 .=L^{\prime o n}$ appelle $\varphi$-algèbre toute $\mathscr{L}$-algèbre régulière $(E, \mathscr{L},$.$) ,$ i.e. telle que :

a) La multiplication soit associative, commutative et tout élément $x \in E-\varphi(\varnothing)$ est régulier.

et b) $\forall x \in E-\varphi(\varnothing), \forall x \in \mathcal{J}(\mathrm{E}), \quad 1$ 'on a :

$$
x \cdot x \in \mathscr{C} \Longleftrightarrow x \in \mathscr{C} \Longleftrightarrow x: x \in \mathscr{C}
$$

exemple.- Soit A une algèbre intègre sur un corps $K$. A est une $\varphi$-algèbre pour:

- $\varphi(X)$ sous-espace vectoriel engendré par $X$

- la multiplication dans A.

Soit $(\bar{E},$.$) le symetrisé de E$ pour la multiplication, et $G$ $l$ 'ensemble des éléments inversibles de $\bar{E}$. G $\cap E \supset E-\varphi(\varnothing)$.

Soit

$$
\begin{gathered}
\overline{\mathscr{b}}=\left\{\mathrm{x}^{-1} \mathrm{x} \mid \mathrm{x} \in \mathscr{C} \text { et } \mathrm{x} \in \overline{\mathrm{E}}-\varphi(\phi)\right\} \\
\overline{\mathscr{C}} \text { vérifie }\left(\mathrm{c}_{1}\right) \text { et }\left(\mathrm{c}_{2}\right), \quad \bar{C}=\left.\overline{\mathscr{C}}\right|_{\mathrm{E}} \text { et : }
\end{gathered}
$$

$(\bar{E}, \bar{\zeta},$.$) est une \varphi$-algèbre unitaire telle que tout élément $x \notin \varphi(\emptyset)$ soit inversible. C'est la $\varphi$-algèbre symetrisée de $(E, \mathscr{C},$.$) .$

Remarque : Si l'algèbre est unitaire, l'unité $\mathscr{\ell} \notin \varphi(\phi)$, sauf si $\varphi(\varnothing)=$ E.

Soit $A=(E, \varphi,$.$) une \varphi$-algèbre. L'on appelle sous-algèbre de A toute partie $B$ de A telle que :

$$
A=\varphi(A) \text { et A stable pour la multiplication. }
$$

Toute intersection de sous-algèbre est une sous-algèbre, d'où la fermeture $\omega$ : 
$X \longrightarrow \omega(X)$ sous-algèbre engendré par $X$

3.4.3.- Propriété fondamentale de la disjonction dans une $\varphi$-algèbre.

Les propriétés elémentaires ont été vues en 3.4.1.

Leme 2.- Solent $X$ et $X '$ deux parties d'une $\varphi$-algèbre $(E, \varphi,$.$) . Pour que X$ et $X^{\prime}$ solent deux disjointes 11 faut et il suffit qu'il existe une $\varphi$-base $B$ de $X$ et une $\varphi$-base $B^{\prime}$ de $X '$ telles que la famille :

$$
\text { (b.b') } \quad\left[\left(b^{\prime} b^{\prime}\right) \in B \times B^{\prime}\right] \text { soit } \varphi \text {-libre. }
$$

\section{Demonstration.}

La condition est nécessaire par définition. Réciproquement, les propriétés en jeu étant de caractère fini, il suffit de démontrer que :

si $r(X)$ et $r\left(X^{\prime}\right)$ sont finies, et si B (resp. $B^{\prime}$ ) est une $\varphi$-base de $X$ (resp. $X^{\prime}$ ) telle que $\left(b, b^{\prime}\right) \quad\left[\left(b^{\prime} b^{\prime}\right) \in B \times B^{\prime}\right]$ soit $\varphi-l i b r e$, on a :

$$
\begin{aligned}
& \forall Y \in \mathscr{L}, \quad Y \in X . \quad \forall Y \in \in \mathcal{L}, Y \subset X^{\prime}, \\
& \left(y \cdot y^{\prime}\right) \quad\left[\left(y, y^{\prime}\right) \in Y \times Y^{\prime}\right] \quad \text { est } \varphi \text {-libre. }
\end{aligned}
$$

Soit $Z$ (resp. $Z$ ') une $\varphi$-base de $X$ (resp;.X') contenant $Y$ (resp. $Y^{\prime}$ ) .

Card $z=$ Card $B=r(x), \quad$ Card $Z^{\prime}=$ Card $B^{\prime}=\mathbf{r}\left(X^{\prime}\right)$

Or, il est aisé de démontrer que,

si $A_{1}$ engendre $A$ et $s i B_{1}$ engendre $B$,

$$
A_{1} \cdot B_{1} \text { engendre A.B }
$$

Donc $\& \varphi\left(Z_{\text {.Z }}^{\prime}\right)=\varphi\left(\right.$ B.B $\left.^{\prime}\right)=\varphi\left(X_{.} X^{\prime}\right)$

Honc $r\left(2.2^{\prime}\right)=r\left(B^{\prime} B^{\prime}\right)=$ Card B $\times$ Card $B^{\prime}$ 
et comme Card $Z_{. Z^{\prime}} \leq$ Card $Z \times$ Card $Z^{\prime}$,

$$
\left(z \cdot z^{\prime}\right) \quad\left[\left(z, z^{\prime}\right) \in z \times z^{\prime}\right] \text { est libre, }
$$

et a fortiori

$$
\left(y \bullet y^{\prime}\right) \quad\left[\left(y, y^{\prime}\right) \in z \times z^{\prime}\right]
$$

Théorème 6.- Soit $\left(x_{i}\right)_{i \in I}$ une famille de parties d'une $\varphi$-algèbre $A$. Les propriétés suivantes sont équivalentes :

$\left(\begin{array}{lll}2 & D_{1}\end{array}\right) \quad\left(x_{i}\right)$ est 2-disjointe

$\left(\begin{array}{ll}2 & D_{1}\end{array}\right) \quad\left(\varphi\left(x_{i}\right)\right)_{i \in I}$ est 2-disjointe

$\left(\begin{array}{ll}2 & D_{2}\end{array}\right) \quad$ Pour tout $i \in I$, il existe une base $B_{i}$ de $x_{i}$ telle que $\left(B_{i}\right)_{i \in I}$ soit 2-disjointe.

Les propriétés $\left(\begin{array}{ll}2 & D_{1}\end{array}\right) \Longrightarrow\left(\begin{array}{ll}2 & D\end{array}\right)$ et $(2 \mathrm{D}) \rightarrow\left(\begin{array}{ll}2 & D_{2}\end{array}\right)$ résultent trivialement des définitions. La propriété $\left(\begin{array}{ll}2 & D_{2}\end{array}\right) \Rightarrow\left(\begin{array}{ll}2 & D_{1}\end{array}\right)$ se démontre par récurrence sur Card I quand I est fini en utilisant le lemme 2 et l'associativité de la 2-disjonction, puis en utilisant le caractère fini de la 2-disjonction quand I est infini.

Remarque : Si $X$ et $Y$ sont 2-disjoints, en général $\omega(X)$ et $\omega(Y)$ ne le sont pas. Ainsi dans l'algèbre $k[x]$ des polynomes à une indéterminée $x$ sur un corps $k$, $X=\left\{x, x^{2}\right\}$ et $Y=\left\{x^{3}, x^{6}\right\}$ sont 2-disjoints, mais $\omega(x)=x k[x]$ et $\omega(Y)=$ $x^{3} k[x]$ ne sont pas 2-disjoints.

Théorème 7.- Soit $\left(A_{i}\right)_{i \in I}$ une famille de sous-algèbre d'une 4 -algèbre.

$$
A=\prod_{i \in I}^{d} \quad A_{i}=\bigcup_{J \in \mathcal{F}(I)}\left(\prod_{i \in J} A_{i}\right)
$$


est la sous-algèbre engendrée par les $A_{i}$ et (*) l'on a :

$$
\mathbf{r}(\mathrm{A}) \leq \prod_{i \in I} \mathbf{d} \quad \mathbf{r}\left(\mathbf{A}_{\mathbf{i}}\right)
$$

Si les $\left(A_{i}\right)$ sont disjoints, on a l'égalité dans (1).

$$
\text { Réciproquement, si } I \text { et les } r\left(A_{i}\right) \text { sont finis et si la sous- }
$$

algèbre A engendrée par les $A_{i}$ a pour rang :

$$
r(A)=\prod_{i \in I} r\left(A_{i}\right),
$$

alors $\left(A_{i}\right)_{i \in I}$ est 2-disjointe.

La démonstration de ce théorème est analogue à celle du théorème 4 . La réciproque $n$ 'est plus vraie en général quand $I$ ou $l$ 'un au moins des $r\left(A_{i}\right)$ est infini.

L'on a ici une propriété classique de la disjonction linéaire (voir Bourbaki $[\mathrm{C} ; \mathrm{II}, 5$,$] \$2, n॰3 ,P. 78$ à 81).

\subsection{2.- 2-disjonction unitaire}

Théorème 8.- Soit $\left(x_{i}\right)_{i \in I}$ une famille de parties d'une $\varphi$-algèbre unitaire non grossière, d'unité e. Alors les propriétés suivantes sont équivalentes :

(*) Soit $\left(a_{i}\right)_{i \in I}$ une famille de cardinaux

et $\prod_{i \in I}^{d} a_{i}=\left\{\left(x_{i}\right)_{i \in I} \mid \begin{array}{lll}\forall i \in I \quad, \quad x_{i} \in a_{i} \quad \text { ou } \quad x_{i}=0 \\ \text { et } x_{i} \neq 0 \text { pour un nombre fini d'élément }\end{array}\right\}$

ne dépend que de Card $a_{i}$. C'est donc, par abus de langage, un cardinal. 


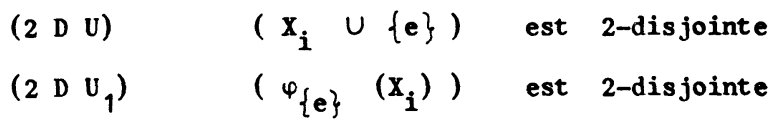

$\left(\begin{array}{lll}2 & \mathrm{D}_{2}\end{array}\right) \quad$ Pour tout $i \in I$, il existe une $\varphi$-base $B_{i}$ de $x_{i} \cup\{e\}$ telle que $e \in B_{i}$ et $\left(B^{\prime}{ }_{i}\right)=\left(B^{\prime}{ }_{i}-\{e\}\right)$ vérifie la propriété :

(DU) $\quad\left(\prod_{i \in J} b_{i}\right) \quad\left[\left(b_{i}\right) \in \cup \prod_{i \in J} B_{i}^{\prime} \quad[J \in \mathcal{F}(I)]\right]$ est libre

Démonstration

L'on a $(2 \mathrm{D} U) \Longleftrightarrow\left(2 \mathrm{D} \mathrm{U}_{1}\right)$ d'après le théorème 6 . Nous allons démontrer que $\left(2 \mathrm{D} \mathrm{U}_{2}\right) \Longleftrightarrow\left(\begin{array}{lll}2 & \mathrm{D} & \mathrm{U}\end{array}\right)$ :

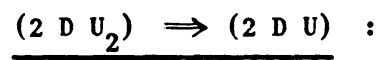

Soit $\left(X_{i}\right)$ une famille vérifiant $\left(\begin{array}{lll}2 & D & U_{2}\end{array}\right)$ et $\left(B_{i}\right)$ incluse dans $\left(x_{i} \cup\{e\}\right)$ telle que pour tout $i$ e $\in B_{i}$ et $\left(B^{\prime}{ }_{i}\right)=\left(B_{i}-\{e\}\right)$ vérifie la propriété (DU). Alors, pour tout $J \in \mathcal{F}(I)$,

$\left(\prod_{i \in J} B_{i}\right) \quad\left[\left(b_{i}\right) \in \prod_{i \in J} B_{i}\right]=\left(\prod_{i \in H} b_{i}\right)\left[\left(b_{i}\right) \in \cup \prod_{i \in H} B_{i}^{\prime}[H \subset J]\right]$

donc, est libre d'après (DU). Par conséquent $\left(B_{i}\right)_{i \in I}$ est 2-disjointe et $\left(x_{i} \cup\{e\}\right)$ est 2-disjointe d'après le théorème 6.

$\left(\begin{array}{lll}2 & \mathrm{D} & \mathrm{U}\end{array}\right) \Longrightarrow\left(\begin{array}{lll}2 & \mathrm{D} & \mathrm{U}_{2}\end{array}\right) \quad$ se démontre de manière analogue.

\section{Corollaire 1.-}

Soit $\left(X_{i}\right)$ une famille de parties d'une $\varphi$-algèbre $(E, \varphi,$.$) et (\bar{E}, \bar{\varphi},$. la $\varphi$-algèbre symetrisée de $(E, \varphi,$.$) , dont l'unité est e. Alors les propriétés$ suivantes sont équivalentes :

(2 D U ) ( $\left.x_{i} \cup\{e\}\right)$ est 2-disjointe dans $(\bar{E}, \bar{\varphi},$.

( $2 \mathrm{D}_{3}$ ) Pour tout $i \in I$, il existe une $\varphi$-base $B_{i}$ de $x_{i}$ (resp. si $(E, \varphi,$. est unitaire, il existe une $\varphi\{e\}$-base $B_{i}$ de $\left.x_{i}\right)$ telle que $\left(B_{i}\right)$ verifie la 
propriété (D U) ci-dessus.

Definition 4:- Une famille $\left(x_{i}\right)_{i \in I}$ de parties d'une $\varphi$-algèbre est dite

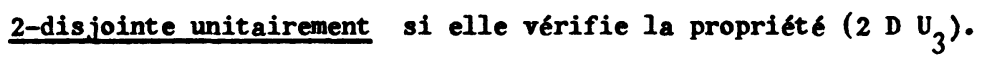

Corollaire 2.- Si $\left(X_{i}\right)_{i \in I}$ est 2-disjointe et si $\left(Y_{i}\right)$ est incluse dans $\left(X_{i}\right)$ et vérifie :

$\forall i \in I, \quad Y_{i}$ est une $\varphi$-base (resp. si $E$ est unitaire une $\varphi\{e\}^{\text {-base) }}$ de $\mathrm{X}_{i}, \quad\left(\mathrm{Y}_{i}\right)$ vérifie la propriété (D U).

Corollaire $3 .-$ Si $\left(x_{i}\right)$ est 2-disjointe unitairement, $\left(x_{i}\right)$ est $\mathscr{L}[\varepsilon]$-disjointe.

Si la $\varphi$-algèbre est unitaire, $\varphi_{e}$ désigne la translatée de $\varphi$ par $\{e\}$, et si la $\varphi$-algèbre n'est pas unitaire, $\varphi_{\mathrm{e}}=\varphi_{\mathrm{A}}$ où :

$A=\bar{\varphi}(e) \cap E=\{x \mid x \in E$ et $\exists y \in E-\varphi(\varnothing),\{y x, y\} \in \mathscr{C}\} \cup \varphi(\varnothing)$.

Ce corollaire résulte des théorèmes 1 et 8 sachant que, dans (D U),

$$
\left(\prod_{i \in J} b_{i}\right) \quad\left[\left(b_{i}\right) \in \quad \cup \quad \prod_{i \in J} B^{\prime}{ }_{i} \quad\left[J \in F_{1}(I)\right]\right]
$$

est la famille somme des familles $\left(b_{i}\right)_{i \in B^{\prime}}{ }_{i}$

\section{Remarque 1.-}

La 2-disjonction unitaire est strictement plus forte que la 2-disjonction.

exemple : Soit $k[x]$ l'algèbre des polynomes une indéterminée sur un corps $k$. $H \subset N$ et $K \subset N$ ( $N$ ensemble des entiers naturels)

$$
X=\left\{x^{p} \mid p \in H\right\} \quad \text { et } \quad Y=\left\{x^{p} \mid p \in K\right\}
$$

$X$ et $Y$ sont 2-disjoints si et seulement si l'application $(p, q) \longrightarrow p+q$ de $H \times K$ dans $N$ est injective. 
L'on vérifiera aisément par exemple que :

$$
X=\left\{x, x^{3}\right\} \quad \text { et } Y=\left\{x^{2}\right\} \text { sont 2-disjoints, }
$$

mais qu'ils ne sont pas unitairement 2-disjoints.

Peararque 2. Nême si $X$ et $Y$ sont unitairement disjoints dans $(\bar{E}, \bar{\varphi},),. \quad X \cup x^{-1}$ et $Y \cup Y^{-1}$ ne sont pas 2-disjoints en général.

(considérer par exemple $X=\{1, x\}$ et $Y=\left\{1, x^{2}\right\}$ dans $I$ 'exemple ci-dessus).

Reangue 3. La 2-disjonction unitaire est strictement plus forte que la $\mathscr{L}$ [e]-disjonction. En effet dans l'exemple ci-dessus, $X$ et $Y$ sont $\mathscr{L}[e]$-disjoints si et seulement si $\mathrm{H} \cap \mathrm{K} \subset\{0\}$.

Renaque 4.- Si $X$ et $Y$ sont 2-disjoints unitairement, en général $X$ et $\omega(Y)$ ne sont mime pas 2-disjoints. 
Chapitre IV Existence de familles disjointes incluses dans une famille finie donnée.

\section{1.- Notations et terminologie générales}

$E$ étant un $\varphi$-espace et $I$ un ensemble d'indices, on tutilisera couranment les notations suivantes :

$1^{\circ}$ ) Les lettres $\mathcal{A}, B, \mathscr{C}, \ldots$ désignent des familles de cardinaux, et si $A=\left(a_{i}\right)_{i \in I}$, pour tout $\mathrm{JCI}$, on note :

$$
\underline{a_{J}=\Sigma_{a_{i}}[i \in J] \quad \text { et } \quad t_{J}=\left(a_{i}\right)_{i \in J}}
$$

20) $\left(X_{i}\right)_{i \in I},\left(Y_{i}\right),\left(z_{i}\right)$ désignent des familles de parties de $E$ et pour tout $\mathrm{J} \subset \mathrm{I}, \mathrm{x}_{\mathrm{J}}=\mathrm{U} \mathrm{x}_{\mathrm{i}} \quad[\mathrm{i} \in \mathrm{J}]$.

On note $\left(Y_{i}\right)_{[i \in I]} \subset\left(X_{i}\right)[i \in I]$ l'ordre produit sur $(Z(E))^{I}$, et $A_{b} \leq l^{\prime}$ ordre produit sur les familles de cardinaux indiciées par I ( $A$ inférieure ou égale à $\beta$ si et seulement si $a_{i} \leq b_{i}$ pour tout $\left.i\right)$.

On a vu que si $\left(X_{i}\right)_{i \in I}$ contient une famille $\varphi$-disjointe $\left(Y_{i}\right)$ telle que $\operatorname{dim} \varphi\left(Y_{i}\right)=a_{i}$ pour tout $i$, on a :

$$
\forall \mathrm{J} \subset \mathrm{I} \quad \operatorname{dim} \varphi\left(\mathrm{x}_{\mathrm{J}}\right) \geq \mathbf{a}_{\mathrm{J}}
$$

Définition 1.- Une famille $\left(x_{i}\right)_{i \in I}$ est dite de type $\underline{f_{b}}=\left(a_{i}\right)_{i \in I}$ si elle possède la propriété suivante :

$$
P_{\text {of }}: \forall \mathrm{J} \subset \mathrm{I}, \operatorname{dim}_{\varphi} \varphi\left(\mathrm{x}_{\mathrm{J}}\right) \geq \mathrm{a}_{\mathrm{J}}
$$

Si $a_{I}>\operatorname{dim} E$, il $n$ 'existe aucune famille de type $t_{6}$. Par la suite on supposera la condition $a_{I} \leq$ dim $E$ réalisée.

$$
\text { Soit } A b=\left(a_{i}\right)_{i \in I} \text { et } J \quad l \text { 'ensemble des indices } i \text { tels que } a_{i} \neq 0 \text {, }
$$
il est clair que $\left(x_{i}\right)_{i \in I}$ est de type to si et seulement $s i \quad\left(x_{i}\right)_{i \in J}$ est de type $A_{\mathrm{J}}$. 
Comme $a_{I} \leqslant \operatorname{dim} \varphi, \quad a_{i} \leq \operatorname{dim} E$ pour tout $i . S i \mathcal{N}_{E}$ est $I$ 'ensemble des cardinaux inférieurs ou égaux à $\operatorname{dim} E, \mathscr{f} \in\left(\not \mathbb{P}_{E}\right) \mathrm{I}$, de sorte que les familles de cardinaux qui nous sont utiles appartiennent à un ensemble.

Une famille $\varphi$-disjointe $\left(x_{i}\right)_{i \in I}$ est de type $t=\left(a_{i}\right)$ si et seulement si pour tout $i \operatorname{dim} \varphi\left(x_{i}\right) \geq a_{i}$ (Cf. 3.3, théorème 4). On constate par ailleurs que : si $\left(x_{i}\right)_{i \in I}$ est de type $t$, toute famille $\left(Y_{i}\right)$ contenant $\left(x_{i}\right)$ est de type of

; $\left(x_{1}\right)$ est de type $\beta$. pour toute $\beta$ inférieure ou égale à ${ }^{\circ}$, et pour tout $J \subset I,\left(x_{i}\right)[i \in J]$ est de type of t $_{\mathrm{J}}$

En particulier,toute famille contenant une famille $\varphi$-disjointe de type of est elle-meme de type $t \hat{t}$. C'est la réciproque quand $I$ ou les $a_{i}$ sont finis : qui est l'objet de ce chapitre : étant donné une famille de trpe of, existe-t-il une famille $\varphi$-disjointe, incluse, de type to, et si oui en construire une.

4.2.- Cas de familles finies de cardinaux finis.

Dans ce paragraphe nous allons démontrer dans le cas où les cardinaux $a_{i}$ sont tous finis le théorème suivant :

Théorème 1.- Toute famille finie de type of contient une famille $\varphi$-disjointe de type of.

On indiquera dans le prochain paragraphe comment résoudre ce problème en pratique. Enfin le cas où les $a_{i}$ soṇt quelconques sera étudié au paragraphe 4.4. 
Le théorème 1 est une généralisation du théorème de KönigłHa]l(voir [B], chap. 10, page 02 ) qui traite le cas d'un ensemble, i.e. $\varphi$-espace discret, I fini et les $a_{i}$ tous égaux à $1(*)$.

M. Rado dans [9] a également démontré un cas particulier du théorème 1 : les $a_{i}$ tous égaux à 1 et $\left(x_{i}\right)_{i \in I}$ famille de parties d'un ensemble muni d'une structure équivalente à celle de $\varphi$-espace.

Soit $\left(x_{i}\right)[i \in I]$ une famille de type $t=\left(a_{i}\right)$. Pour trouver une solution c'est-à-dire une famille $\left(Y_{i}\right) \varphi$-disjointe, de type of et incluse dans $\left(x_{i}\right)$, nous allons donner deux procédés : $l^{\prime}$ un consiste à supprimer des éléments aux $\mathrm{X}_{i}$ tout

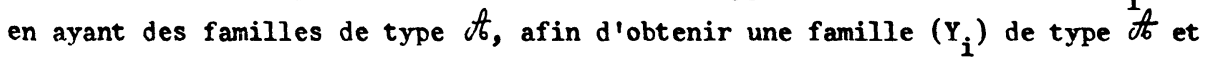
telle que $\operatorname{dim} \varphi\left(Y_{i}\right)=a_{i}$ pour tout $i$. Il en résulte que $\operatorname{dim} \varphi\left(Y_{I}\right)=a_{I}$ (voir 3.3 théorème 4$)$, donc $\left(Y_{i}\right)$ est $\varphi$-disjointe (voir 3.3., corollaire du théorème 4). Dans le second procédé on part de la famille $(\varnothing)$ et on construit des familles $\varphi$-disjointes $\left(Y_{i}\right)$, chaque $Y_{i}$ étant une partie libre de $X_{i}$, en ajoutant des éléments aux $Y_{i}$. Le théorème 1 étant trivial si Card $I=1$, on supposera Card $I \geq 2$.

a) - Démonstration par suppressibilité

Remarquons d'abord que $\left(x_{i}\right)_{i} \in I$ étant de type $t b=\left(a_{i}\right)$, toute famille $\left(z_{i}\right)$ incluse dans $\left(x_{i}\right)$ telle que :

$\begin{array}{lll}\operatorname{dim} \varphi\left(z_{i}\right)=\operatorname{dim} \varphi\left(x_{i}\right) & \text { si } & \operatorname{dim} \varphi\left(x_{i}\right)<a_{I} \\ \text { et } \quad \operatorname{dim} \varphi\left(z_{i}\right) \geq a_{I} & \text { si } & \operatorname{dim} \varphi\left(x_{i}\right) \geq a_{I}\end{array}$

est de type $t$ t ; on peut donc réduire $\left(x_{i}\right)$ à une famille incluse $\left(z_{i}\right)$ de type $\mathscr{A}$ et telle que dim $\varphi\left(z_{i}\right) \leqslant a_{I}$ pour tout $i$. Il nous suffit donc de démontrer le

(*)- J'ai résolu ce problème alors que j'ignorais le théorème de König-Hall et les travaux de $M$. Rado.

L'intérét de ce $\$ 1$ est avant tout sa nouveauté, d'abord en ce qui concerne la généralité du résultat, mais surtout dans la manière de traiter ce problème en travaillant avec des méthodes constructives, et en étudiant certaines propriétés. Voici quelques références pour les questions de priorité scientifique concernant la "genèse" de ce théorème :

et enfin Robert P. $[14]$.

$$
\begin{aligned}
& \text { - Konig O. et Valko S. [S] } \\
& \text { - Hall P. [1] } \\
& \text { - Rado R. [9] et [10] }
\end{aligned}
$$


théorème 1 quand les $\operatorname{dim} \varphi\left(x_{i}\right)$ sont finis, ce que nous allons faire par récurrence sur $a_{I}$.

Le théorème 1 est trivial pour $a_{I}=0$. Supposons le vrai pour $a_{I} \leqslant n-1$, et soit $\left(x_{i}\right) \quad[i \in I]$ une famille de type $t=\left(a_{i}\right)$ où $a_{I}=n$ et où les $\operatorname{dim} \varphi\left(x_{i}\right)$ sont finis. Nous allons démontrer que :

$\left(R_{f b}\right):\left[\right.$ Pour tout $i \in I$ tel que $\operatorname{dim} \varphi\left(x_{i}\right)>a_{i}$, il existe $\left(z_{j}\right)[j \in I]$ de type of telle que : $z_{j}=x_{j} \quad \forall j \in I-\{i\}$ et $z_{i} \subset x_{i}$, $\operatorname{dim} \varphi\left(z_{i}\right)<\operatorname{dim} \varphi\left(x_{i}\right)$

Soit en effet $i \in I$ tel que $\operatorname{dim} \varphi\left(X_{i}\right)>a_{1}$

ou $a_{i}=0:$ il suffit de prendre $z_{i}=\varnothing$

ou $a_{i}=1$ : Soit $T_{i}$ une base de $\varphi\left(x_{i}\right)$ incluse dans $x_{i}$. Il nous suffit de démontrer qu'il existe un élément $x$ de $T_{i}$ "suppressible", i.e. tel qu'on peut prendre $z_{i}=T_{i}-\{x\}$. Il est aisé de voir que $x \in T_{i}$ est suppressible si et seulement si : $\forall J \subset I-\{x\}, \operatorname{dim} \varphi\left(x_{J} \cup\left(T_{i}-\{x\}\right)\right) \geq a_{J}+1$. Comme $\operatorname{dim} \varphi\left(x_{i}\right)=\operatorname{Card} T_{i}>1$, soient $x$ et $y$ deux éléments distincts de $T_{i}$. Si $x$ et $y$ sont non suppressibles, il existe $J \subset I-\{i\}$ et $H \subset I-\{i\}$ tels que :

$\operatorname{dim} \varphi\left(x_{J} \cup\left(T_{i}-\{x p)<a_{J}+1\right.\right.$ et $\operatorname{dim} \varphi\left(x_{H} u\left(T_{i}-\{y\}\right)\right)<a_{H}+1$.

or $\operatorname{dim} \varphi\left(x_{J}\right) \geq a_{J}, \operatorname{donc} \operatorname{dim} \varphi\left(x_{J} \cup\left(T_{i}-\{x\}\right)\right)=\operatorname{dim} \varphi\left(x_{J}\right)=a_{J}$ et $T_{i}-\{x\} \subset \varphi\left(x_{J}\right)$. De même $\operatorname{dim} \varphi\left(x_{H}\right)=a_{H}$ et $T_{i}-\{y\} \subset \varphi\left(x_{H}\right)$. D'où $\operatorname{dim} \varphi\left(x_{J \cup H}\right)=a_{J \cup H}\left(\forall i \in J \cup H, \operatorname{dim} \varphi\left(X_{i}\right)=a_{i}\right)$, et $T_{i} \subset \varphi\left(X_{J \cup H}\right)$, de sorte que $\operatorname{dim} \varphi\left(X_{J \cup H} \cup X_{i}\right)=a_{J \cup H}<a_{J \cup H}+a_{i}:\left(X_{i}\right)$ ne serait pas de type $A^{A}$. L'un des deux eléments $x$ et $y$ est donc suppressible.

ou $a_{i} \geq 2$ : Soit $B=\left(b_{j}\right)$ et $\ell=\left(c_{j}\right)$ ou $b_{j}=c_{j} \quad \forall j \in J-\{i\} \quad$ et $b_{i}=\overline{a_{i}-1 \geq 1}, \quad c_{i}=1$. Comme $b_{I}=n-1$, il existe une famille disjointe ( $\left.U_{j}\right)$ incluse dans $\left(x_{j}\right)$ telle que $\operatorname{dim} \varphi\left(U_{j}\right)=b_{j}$ pour tout $j$ dans $I$. Dans $E$ considéré come $\psi$-espace où $\psi=\varphi_{U_{i}},\left(x_{j}\right)$ est de type $\mathscr{C}:$ soit $J \subset I$, ou $i \notin J$ et $\operatorname{dim}_{\psi} \Psi\left(x_{J}\right) \geqslant \operatorname{dim}_{\psi} \Psi\left(Y_{J}\right)=b_{J}=c_{J}$, ou $i \in J$ et $\operatorname{dim}_{\psi} \psi\left(x_{J}\right)=\operatorname{dim}_{\varphi} \varphi\left(x_{J}\right)-\operatorname{dim}_{\varphi} \varphi\left(U_{i}\right) \geq a_{J}+1-a_{i} \geq c_{J} \cdot$ (c.f. 3.3. théorème 4 et son corollaire). Comme $c_{I} \leqslant n-1, \exists\left(v_{j}\right) \subset\left(x_{j}\right)$, 
$\left(v_{j}\right) \Psi$-disjointe et $\operatorname{dim}_{\psi} \psi\left(\nabla_{j}\right)=c_{j} \quad \forall j \in I$. Soit pour tout $j \quad w_{j}$ une $\psi$-base de $\psi\left(v_{j}\right)$ incluse dans $v_{j}$. En posant $T_{j}=w_{j}$ si $j \neq i$ et $T_{i}=w_{i} \cup U_{i}$, $\left(r_{j}\right)[j \in I]$ est $\varphi$-disjointe, de type $A_{f}$ et incluse dans $\left(x_{j}\right)$. Il nous suffit donc de prendre $z_{i}=T_{i}$ dans $R_{f_{t}}$.

Remarquons ici que 1 'hypothèse de récurrence ne nous sert pas pour $a_{i}=0$ et $a_{i}=1$, et sert à trouver une solution directement $\left(\left(z_{i}\right)\right.$ est de type to et $\varphi$-disjointe) quand $a_{i} \geq 2$.

Par au plus $\sum\left(\operatorname{dim} \varphi\left(x_{i}\right)-a_{i}\right)[i \in I]$ opérations de suppression comme celle indique dans $R_{f t}$, on obtiénttune famille $\left(Y_{i}\right)_{i \in I}$ de type of et telle que $\operatorname{dim}_{\varphi} \varphi\left(Y_{i}\right)=a_{i}$ pour tout $i .\left(Y_{i}\right)$ est donc solution (c.f. 3.3. corollaire du théorène 4).

\section{b) - Demonstration par empilement}

Le première démonstration quí repose sur la suppressibilité de certains éléments exige en pratique beaucoup de vérifications d'inégalités. Il sera souvent plus aisé d'ajouter des éléments les uns après les autres, en faisant des permutations quand besoin est.

(R' $\left.R_{f}\right) \quad\left[\begin{array}{c}\text { Soit }\left(x_{i}\right)[i \in I] \text { une familile de type } A=\left(a_{i}\right)[i \in I] \text {, on a : } \\ : \text { soit }\left(Y_{i}\right)[i \in I] \varphi \text {-disjointe, où chaque } Y_{i} \text { est une partie }\end{array}\right.$ libre de $X_{i}$ et Card $Y_{i} \leqslant a_{i}$. Pour tout $i \in I$ tel que Card $Y_{i}<a_{i}$, il existe $\left(z_{j}\right) \varphi$-disjointe, où chaque $z_{j}$ est une partie libre de $x_{j}$ et $\operatorname{Card} z_{j}=\operatorname{Card} Y_{j}$ si $j \neq i$, et $z_{i}>Y_{i}, \operatorname{Card} z_{i}>\operatorname{Card} Y_{i}$.

$n$ est clair que, partant de la famille $(\phi)$, par au plus a opérations come celle indiquée dans $R$ ' ff on construit une solution pour $\left(x_{i}\right)$.

Soit en effet $i \in I$ tel que Card $Y_{i}<a_{i}$. Si $X_{i} \notin \varphi\left(Y_{I}\right)$, il suffit de prendre $z_{j}=Y_{j} \forall j \in I-\{i\}$ et $z_{i}=y_{i} \cup\{x\}$ où $x \in X_{i}, x \notin \varphi\left(Y_{I}\right)$. Mais en général $x_{1} \subset \varphi\left(Y_{I}\right)$. On considère alors les suites récurrentes : 


$$
\begin{aligned}
& K_{0}=I-\{i\} \quad I_{0}=K_{0} U\{i\}=I, \quad Y^{\bullet}=U U_{i}\left[i \in I_{0}\right] \\
& K_{1}=\left\{j / j \in K_{0}, X_{j} \in \varphi\left(Y^{0}\right)\right\} \quad, I_{1}=K_{1} \cup\{i\} \quad, \quad Y^{1}=\cup Y_{i}\left[i \in I_{1}\right] \\
& \text { •. } \quad \text {.. } \quad \text {.. } \\
& K_{p}=\left\{j / j \in K_{p-1}, X_{j} \subset \varphi\left(Y^{p-1}\right)\right\}, \quad I_{p}=K_{p} \cup\{i\}, \quad Y^{p}=U Y_{i}\left[i \in I_{p}\right] \\
& \text { •・ }
\end{aligned}
$$

D'où les suites décroissantes : $K_{\bullet} \supset K_{1} \supset \ldots \supset K_{p-1} \supset K_{p} \supset \ldots$

$$
\text { et } \quad Y^{0} \supset Y^{1} \supset \ldots>Y^{p-1} \supset Y^{p} \supset \ldots
$$

Pour tout entier $p>0$ 'tel que $x_{1} \subset \varphi\left(y^{p}\right)$, on a $y^{p+1} \neq y^{p}$. En effet si $Y^{p+1}=Y^{p}, K_{p+1}=K_{p} \quad\left(s i Y_{j}=\varnothing, j \in K_{q} \quad \forall q \geq 0 ; s i \quad Y_{j} \neq \emptyset,\left(Y_{j}\right)\right.$ étant disjointe, les $Y_{j}$ sont mutuellement disjoints), donc :

$$
\forall j \in I_{p}=I_{p+1}, X_{j} \subset \varphi\left(Y^{p}\right): \varphi\left(X_{I_{p}}\right) \subset \varphi\left(Y^{p}\right)
$$

on aurait donc $\operatorname{dim} \varphi\left(x_{I_{p}}\right)<\sum a_{i}\left[i \in I_{p}\right],\left(x_{i}\right)$ ne serait donc pas de type $f$. Si $x_{i} \subset \varphi\left(Y^{p}\right)$ pour tout $p \geq 0$, on aurait une suite infinie strictement décroissante de parties de $I:\left(K_{p}\right)$. Or I est fini, donc il existe un entier $q$ tel que : $\mathrm{X}_{i}<\varphi\left(Y^{p}\right)$ si $0 \leqslant p<q$ et $x_{i} \notin \varphi\left(Y^{q}\right)$. Les suites finies $\left(Y^{p}\right) \quad 0 \leqslant p \leqslant q$ et $\left(K_{p}\right) \quad 0 \leqslant p \leqslant q$ sont donc strictement décroissantes. Nous allons démontrer que :

$$
\begin{array}{lll}
\forall x \in X_{i}-\varphi\left(Y^{q}\right) & , \exists i(q-1) \in K_{q-1}-K_{q}, & \exists e_{q-1} \in Y_{i(q-1)}, \\
\forall \varepsilon_{q-1} \in X_{i(q-1)}-\varphi\left(Y^{q-1}\right) & , \exists i(q-2) \in K_{q-2}-K_{q-1}, \exists e_{q-2} \in Y_{i(q-2)},
\end{array}
$$$$
\forall \varepsilon_{q-k} \in X_{i(q-k)}-\varphi\left(Y^{q-k}\right) \quad, \quad \exists i(q-k-1) \in K_{q-k-1}-K_{q-k}, \exists e_{q-k-1} \in Y_{i(q-k-1)},
$$$$
\forall \varepsilon_{0} \in \mathbf{X}_{\mathbf{i}(0)}-\varphi\left(\mathbf{Y}^{0}\right) \text {, en posant : }
$$

$$
z_{i}=Y_{i} \cup\{x\}, z_{i(k)}=\left(Y_{i(k)}-\left\{e_{k}\right\} \cup\left\{\varepsilon_{k}\right\} \text { pour tout entier } k\right. \text { tel }
$$

que $0 \leqslant k \leqslant q-1$, et $z_{j}=Y_{j}$ pour les autres indices, on obtient une famille demandée pour R'to . 
En effet, comme $X_{i} \notin \varphi\left(Y^{q}\right)$, soit $x \in X_{i}-\varphi\left(Y^{q}\right)$. Considérant $E$ comme $\psi$-espace où $\psi=\varphi_{Y q},\{X\}$ est $\psi$-libre. or $Y^{q-1}-Y^{q}$ est une $\psi$-base de $\Psi\left(Y^{q-1}\right)=\varphi\left(Y^{q-1}\right)$, on peut échanger $x$ et un élément $e_{q-1}$ de $Y^{q-1}-Y^{q}$ pour obtenir une autre $\psi$-base de $\psi\left(Y^{q-1}\right)$, et les $Y_{j}$ étant mutuellement disjoints, il existe $i(q-1)$ unique, $i(q-1) \in K_{q-1}-K_{q}$ tel que $e_{q-1} \in Y_{i(q-1)}$. Comme $i(q-1) \notin K_{q}, \quad x_{i(q-1)} \notin \varphi\left(Y^{q-1}\right)$, et il est alors clair que $\left.: \forall \varepsilon_{q-1} \in \mathbf{x}_{i(q-1)}\right)^{-\varphi\left(Y^{q-1}\right)}$

$\left(z_{j}\right)\left[j \in I_{q-1}\right]$ est $\varphi$-disjointe, Card $z_{i}>\operatorname{Card} Y_{i}$ et $z_{i} \supset Y_{i}$, Card $z_{j}=\operatorname{Card} Y_{j}$ pour $j \neq i$ et les $z_{i}$ sont des parties $\varphi$-libres de $X_{i}$. On démontre de manière absolument identique que $\left(z_{j}\right)\left[j \in I_{q-k}\right]$ est $\varphi$-disjointe,... par récurrence sur $k$ : On suppose la propriété vraie jusqu'd l'entier $k(0 \leq k \leq q-1)$, on raisonne dans $E$ considéré comme $\Psi_{K}$-espace où $\psi_{k}=\varphi_{A}$ et $A=\left(U z_{j}\left[j \in I_{q-k}\right]\right)-$ $\left\{\varepsilon_{q-k}\right\}$, et on échange $x$ et $\varepsilon_{q-k}, x_{i}$ et $x_{i(q-k)}$, q et $q-k, q-1$ et $q-k-1$ dans la démonstration ci-dessus.

\section{3.- Application à la résolution pratique du cas fini}

Voici d'abord le résultat obtenu dans le cas fini :

Corollaire 1.- $\mathscr{b}=\left(a_{i}\right)[i \in I]$ étant une famille finie de cardinaux finis et $I_{1}$ l'ensemble des $i$ tels que $a_{i} \neq 0$ pour qu'une famille $\left(x_{i}\right)[i \in I]$ de parties

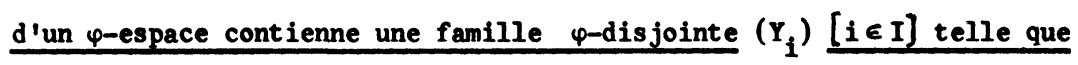
$\operatorname{dim} \varphi\left(Y_{i}\right)=a_{i}$ pour tout $i$, il faut et il suffit que : Pour tout $J \subset I_{1}, \operatorname{dim} \varphi\left(x_{J}\right) \geq a_{J}$

Remarquons que les conditions ( 1 ) quand $J$ décrit l'ensemble des parties non vides de $l$ 'ensemble $I$ des $i$ tels que $a_{i}>0$ sont indépendantes : soit $J$ une telle partie, il existe des familles $\left(x_{i}\right)$ qui vérifient dim $\varphi\left(x_{K}\right) \geq a_{K}$ pour toute partie $K$ différente de $J$ et telles que $\operatorname{dim} \varphi\left(x_{J}\right)<a_{J}$ (à partir d'une famille disjointe $\left(z_{i}\right)$ de parties libres $z_{i}$ telles que Card $z_{i}=a_{i}$, en considérant $x \in z_{J}$ et en prenant $x_{i}=z_{J}-\{x\}$ si $i \in J, x_{i}=z_{i}$ si $i \notin J, \quad\left(x_{i}\right)$ vérifie 
bien $\operatorname{dim} \varphi\left(x_{K}\right)_{2} a_{K}$ pour $K \in I$ et $K \neq J$, et cependant on $\left.a \operatorname{dim} \varphi\left(x_{J}\right)=a_{J}-1 \leq a_{J}\right)$. La condition dim $\varphi\left(\mathrm{X}_{\phi}\right) \geq 0$ est toujours vérifiée. On ne peut donc espérer écrire moins de conditions dans le corollaire.

Maintenant voici comment en pratique on peut résoudre ce problème. Les processus indiqués se programment aisément sur machine dans les cas où $E$ est un $\varphi$-espace discret, ou un espace affin de dimension finie sur un corps fini.

Soit $\left(x_{i}\right)[i \in I]$ la famille à étudier dont on veut trouver une famille $\varphi$-disjointe $\left(Y_{i}\right)$ incluse où $\operatorname{dim} \varphi\left(Y_{i}\right)=a_{i}$ pour tout $i$. Pour tous les indices $i$ tels que $a_{i}=0$, on prend $Y_{i}=\varnothing$ et on les enlève de $I$. Puis on remplace chaque $x_{i}$ pour une base $z_{i}$ de $\varphi\left(x_{i}\right)$ incluse dans $x_{i} \cdot\left(z_{i}\right)$ est de type to car pour tout $\mathrm{J} \subset \mathrm{I}, \quad \varphi\left(z_{\mathrm{J}}\right)=\varphi\left(\mathrm{X}_{\mathrm{J}}\right)$.

On se ramène ainsi systématiquement à des familles de parties libres de type Af telles que $a_{i}>0$ pour tout $i$.

Voici d'abord deux méthodes qui permettent d'obtenir une solution en itérant toujours le même calcul.

a) (suppressibilité).

Soit $\left(x_{i}\right)[i \in I]$ famille de parties libres, de type $t=\left(a_{i}\right) \quad\left(a_{i}>0\right)$. On pose $I=\{1, \ldots, n\}$ et on range les $x_{i}$ de façon que Card $x_{i}-a_{i}$ croisse avec $i$. Pour les $x_{i}$ tels que Card $x_{i}>a_{I}$ on ne garde qu'une partie ayant $a_{I}$ éléments.

Posons $x_{1}=\left\{x_{1}, \ldots, x_{p}\right\}$. Si $p=a_{1}$ on pose $y_{1}=x_{1}$. Sinon $p>a_{1}$ et on vérifie pour $x_{1}$ les $2^{n-1}$ inégalités :

Pour tout $J \subset I-\{1\} \operatorname{dim} \varphi\left(x_{J} \cup\left(x_{1}-\left\{x_{1}\right\}\right)\right) \geq a_{J}+a_{1} \cdot$

Dès que l'une de ces inégalités n'est pas satisfaite, $x_{1}$ 'est pas suppressible et on le garde dans $x_{1}$. Si elles sont toutes vérifiées, $x_{1}$ est suppressible et on l'enlève de $x_{1}$. On obtient ainsi une famille où $x_{1}$ ou bien a p-1 éléments, ou bien a un élément $x_{1}$ non suppressible, cette famille étant toujours de type $d f$. On fait la meme chose pour $x_{2}$ avec la famille obtenue, puis pour $x_{3}, \ldots$ On s'arrête dès que ou bien on a $a_{1}$ éléments non suppressibles, ou bien il ne 
reste que $a_{1}$ éléments dans $x_{1}$ et on pose $Y_{1} l$ 'ensemble de ces $a_{1}$ éléments. Comme $\left(X_{i}\right)[i=2, \ldots, n]$ est de type $t_{I}-\{1\}$ dans $E$ considéré comme $\varphi_{Y_{1}}$-espace; il ne reste qu'à itérer la méthode pour $\left(x_{i}\right)_{i \geq 2}$.

On justifie ce processus de la manière suivante : On sait d'après le théorème 1 qu'il existe une famille $\left(Y_{i}\right)$ de parties libres $Y_{i} \subset X_{i}$, disjointe, et Card $Y_{i}=a_{i}$ pour tout $i$. Si $x \notin Y_{1}$, $x$ est suppressible, de sorte que l'on $a$ au plus $a_{1}$ éléments non suppressibles (ceux de $Y_{q}$ ). S'il y en a $a_{q}$, ils forment $Y_{1}$ de manière unique. S'il $n^{\prime} y$ en $a$ que $b_{1}<a_{1}, Y_{1}$ contient ces éléments et $a_{1}-b_{1}$ éléments suppressibles (on $a c_{p-b}^{a_{1}-b_{1}}$ manièrejde les choisir dans $x_{1}$ ).

b) - (empilement)

Comme en $a)$ on range les $x_{i} i=1, \ldots, n$ de façon que Card $x_{i}-a_{i}$ croisse avec $i$. Puis on prend $Y_{1}^{1}$ partie de $X_{1}$ ayant $a_{1}$ éléments. On construit $\left(\mathrm{Y}_{1}^{2}, \mathrm{Y}_{2}^{2}\right)$ disjointe où chaque $\mathrm{Y}_{i}^{2}$ est une partie libre de $\mathrm{X}_{i}$ ayant $a_{i}$ éléments comme indiqué en 2 b). Supposons que l'on ait ainsi construit de proche en proche $\left(Y_{j}^{i-1}\right) \quad[j=1,2, \ldots, i-1]$ disjointe où les $Y_{j}^{i-1}$ sont des parties libres de $x_{j}$ ayant $a_{j}$ éléments. Pour obtenir $\left(Y_{j}^{i}\right)[j=1,2, \ldots, i]$ on construit $Y_{i}^{i}$ élément par élément par la méthode qui nous a servi à démontrer $\mathbb{R}^{\prime}{ }^{\prime}$. A mesure que $l^{\prime}$ 'on ajoute des éléments pour former $Y_{i}^{i}$, on doit en général modifier les $Y_{j}^{i-1}$ et on barre dans chaque $x_{j}$ les éléments qui ont dâ être enlevés à un $Y_{j}^{i-1}$ à un moment ou à un autre (ce sont des éléments suppressibles au sens de a) ci-dessus), la suite $K_{p} \quad 0 \leq p \leq q$ s'allonge et un indice $j$ donné ne peut passer de $K_{p}$ qu'à un $K_{r}$ où $r \geq p \quad$ (en effet pour tout entier $p, Y^{p}$ croit, donc $K_{p}$ aussi, mais comme on a toujours $x_{i} \subset \varphi\left(Y^{p}\right)$ pour $p<q$, la suite est toujours strictement décroissante Quand $X_{i} \subset \varphi\left(Y^{q}\right)$, on ne peut qu'allonger la suite des $\left.K_{i}\right)$.

c)- remarques

10) Il n'est pas nécessaire de vérifier d'abord que $\left(x_{i}\right)_{i \in I}$ est de type $\overparen{t}$, mais si on le fait, on note les parties $J$ telles que $\operatorname{dim} \varphi\left(x_{J}\right)=a_{J}$ et on ordonne celles-ci suivant Card $J$ croissant : $\left(J_{k}\right)[k=1, \ldots, p]$. 
On résoud $\left(x_{i}\right) \quad\left[i \in J_{1}\right]$, puis on raisonne dans $E$ considéré comme $\varphi_{1}$-espace où $\varphi_{1}=\varphi_{A}$ et $A=U X_{i}\left(i \in J_{1}\right]$. Puis on résoud $\left(x_{i}\right)\left[i \in J_{2}-J_{1} \cap J_{2}\right]$ et on raisonne dans $E$ considéré comme $\varphi_{2}$-espace où $\varphi_{2}=\varphi_{1}$ B et $B=U x_{i}\left[i \in J_{2}-J_{1} \cap J_{2}\right] \quad\left(\varphi_{2}=\varphi_{c} \quad\right.$ où $\left.c=U x_{i}\left[i \in J_{1} \cup J_{2}\right]\right) \ldots$. On obtient en fin de compte une solution partielle $\left(Y_{i}\right)\left[i \in U J_{k}[k=1, \ldots, p]\right]$ dont on sait qu'elle se prolonge en une solution pour $\left(X_{i}\right)$. On est alors ramené au cas général en raisonnant dans $E$ considéré conme $\varphi_{\mathrm{p}}$-espace.

20) La méthode par suppressibilité est avantageuse quand les dim $\varphi\left(x_{i}\right)$ sont voisins des $a_{i}$ car on a alors des chances de supprimer rapidement assez d'éléments. Au contraire plus les dim $\varphi\left(x_{i}\right)$ sont grands et voisins de $a_{I}$, mieux la seconde méthode est heureuse, car on construit rapidement des familles dis jointes incluses de type $b=(b i)$ où $a_{I}-b_{I}$ est petit, la difficulté est reportée sur les derniers éléments (le cas le plus favorable est évidemment celui où dim $\varphi\left(X_{i}\right) \geq a_{I}$ pour tout $i$. Les constructions par la seconde méthode se font alors sans permutation).

$\left.3^{\circ}\right)$ Si on a une famille $\left(x_{i}\right)$ dont on ne sait si elle est de type to ou non (vérifier quand meme dim $\varphi\left(X_{i}\right) \geq a_{i}$ et $\left.\operatorname{dim} \varphi\left(X_{I}\right) \geq a_{I}\right)$, on peut cependant essayer l'une des méthodes a) et $b)$ ci-dessus. Si les calculs aboutissent, c'est que $\left(x_{i}\right)$ est de type of et on a une solution, sinon c'est que $\left(x_{i}\right)$ n'est pas de type of, et il faut alors modifier of (diminuer certains $a_{i}$, en augmenter d'autres...) si on veut des solutions "au mieux", i.e. telles que of soit maximale.

\section{4.- Propriétés générales des familles de type of. Applications}

\subsection{1.- Distributivité des familles de type $A$.}

La remarque qui suit le corollaire nous permet d'affirmer que la propriété pour une famille $\left(x_{i}\right)$ d'être de type tón n'est pas associative (i.e. $\left(J_{l}\right) l \in I$ étant une partition de $I$, si $\left(x_{i}\right)[i \in I]$ est de type $t f=\left(a_{i}\right)\left[i \in I_{b},\left(x_{i}\right)\left[i \in J_{l}\right]\right.$ est de type $C_{\mathrm{J} l}$ pour tout $l$ et $\left(x_{J}\right)[\ell \in L]$ est de type $B=\left(b_{l}=a_{J}\right)$; mais la réciproque est fausse). On a cependant la propriété de "distributivité" suivante : 
Soit $\left(x_{i}\right)[i \in I]$ une famille de type $A=\left(a_{i}\right)$ et pour tout $i$ $a_{i}=\sum b_{\ell}\left[\ell \in L_{i}\right]$. Soit de plus $L l^{\prime}$ 'ensemble somme des $L_{i}$ et pour tout $\ell$ dans $L \quad Y_{\ell}=x_{i}(\ell)$ où $i(\ell)$ est l'indice $i \in I$ unique tel que $l \in I_{i}(l)$. Il est alors aisé de vérifier que :

$$
"\left(x_{i}\right)[i \in I] \text { est de type ot }=\left(a_{i}\right) " \Leftrightarrow "\left(Y_{\ell}\right)[l \in L] \text { est de type } k=\left(b_{\ell}\right) "
$$

Résoudre $\left(\mathrm{X}_{\mathbf{i}}\right)$ et résoudre $\left(\mathrm{Y}_{\ell}\right)$ sont donc deux problèmes équivalents. Voici comment on passe des solutions de $\left(x_{i}\right)$ à celles de $\left(Y_{\ell}\right)$ et réciproquement : $\left(X^{\prime}{ }_{i}\right)$ étant une solution de $\left(x_{i}\right)$, soit pour tout $i x^{\prime \prime}{ }_{i}$ une base de $\varphi\left(X^{\prime}{ }_{i}\right)$ inclase dans $x_{i} \cdot$ Comme Card $x_{i}^{\prime \prime}=p_{i} \geq a_{i}$, il existe

$$
c_{p_{i}}^{a_{i}} \times\left(a_{i} !\right) / \pi\left(b_{\ell} !\right) \quad\left[l \in L_{i}\right]
$$

partitions $\left(\mathrm{Y}_{\ell}^{\prime}\right)\left[\ell \in \mathrm{L}_{\mathbf{i}}\right]$ de $\mathrm{x}_{\mathbf{i}}^{\prime \prime}$ telles que Card $\mathrm{y}_{\ell}^{\prime}=\mathrm{b}_{\ell}$ pour tout $\ell$ dans $\mathrm{L}_{\mathbf{i}}$, et $\left(Y_{\ell}^{\prime}\right) \quad(l \in L)$ est solution pour $\left(Y_{\ell}\right)$. Inversement si $\left(Y_{\ell}^{\prime}\right) \quad[\ell \in L]$ est une solution de $\left(Y_{\ell}\right),\left(Y_{L_{i}^{\prime}}^{\prime}\right)[i \in I]$ est une solution pour $\left(X_{i}\right)$.

Notons que la propriété de distributivité permet de se ramener systématiquement à des familles de type $\mathscr{C}=\left(c_{k}\right)$ où $c_{k}=1$ pour tout $k$. Mais la résolution de ces systèmes est en général beaucoup plus longue que celle du système demandé. On a d'ailleurs toujours avantage à diminuer le nombre d'éléments de la famille, c'est-à-dire à utiliser la distributivité en sens inverse.

\subsection{2. - Familles réduites.}

Soit $\left(x_{i}\right)[i \in I]$ une famille de type $t=\left(a_{i}\right)$. La relation sur $I: R\{i, j\}$ si et seulement si $\varphi\left(x_{i}\right) \subset \varphi\left(x_{j}\right)$ est une relation de préordre. Soit $\rho$ l'équivalence associée, $I_{0}=I / \rho$ est ordonné par la relation d'ordre quotient. On note $\alpha, E, \gamma$ les éléments de $I_{0}$, i.e. les classes d'équivalences de I suivant $\rho$ et $\mathrm{x}_{\alpha}=U \mathrm{x}_{\mathrm{i}}[\mathrm{i} \in \alpha]$.

$$
\text { La famille }\left(\mathrm{x}_{\alpha}\right) \quad\left[\alpha \in \mathrm{I}_{0}\right] \text { est dite réduite de }\left(\mathrm{X}_{\mathrm{i}}\right)
$$

il est c]air que :

$"\left(x_{i}\right)[i \in I]$ est de type $\overrightarrow{t b}=\left(a_{i}\right) " \Longleftrightarrow "\left(x_{\alpha}\right)\left[\alpha \in J_{0}\right]$ est de type $\quad b_{1}=\left(a_{\alpha}\right) "$. 
(on obtient ici une propriété plus forte que la distributivité).

De toute solution $\left(Y_{\alpha}\right)$ de $\left(x_{\alpha}\right)$ on déduit une solution de $\left(x_{i}\right)$ de la manière suivante : Soit $\alpha \in I_{0}$ et $E$ considéré comme $\psi$-espace où $\psi=\varphi_{c}$ et $C=U Y_{\beta}\left[\beta \in I_{0}-\{\alpha\}\right]$. Pour tout $i$ dans $\alpha, \quad \psi\left(Y_{\alpha}\right) \subset A=\psi\left(x_{i}\right)$. Soit donc $z_{1}$ partie $\psi$-libre de $X_{1}$ ayant $a_{1}$ éléments, $z_{2}$ partie $\psi_{Y_{1}}$-libre de $x_{2}$ ayant $a_{2}$ éléments,... (où $\alpha=\{1,2, \ldots\}) .\left(z_{i}\right)_{i \in \alpha}$ est $\psi$-disjointe et $z_{\alpha}$ est $\psi$-libre, et en remplaçant $Y_{\alpha}$ par $z_{\alpha}$ dans la famille $\left(Y_{\beta}\right)$, on a encore une solution pour $\left(\mathrm{x}_{\alpha}\right)$. A partir de cette solution on fait conme ci-dessus avec un second élément de $I_{0}, \ldots$ d'où en fin de compte une famille $\left(z_{i}\right)_{i \in I}$ telle que $\left(z_{\alpha}\right)[\alpha \in I]$ est solution pour $\left(x_{\alpha}\right)$ et $\left(z_{i}\right)[i \in \alpha]$ est solution pour $\left(x_{i}\right)[i \in \alpha]$ d'où (associativité de la disjonction) $\left(z_{i}\right)_{i \in I}$ solution pour $\left(x_{i}\right)$.

\section{5.- Cas de familles finies de cardinaux quel conques}

Soit $\mathcal{A}=\left(a_{i}\right)[i \in I]$ une famille finie de cardinaux. $L=\left\{e_{2}, \ldots, e_{n}\right\}$ étant l'ensemble des cardinaux infinis $a_{i}$ de $I$ rangés dans l'ordre croissant, soit $I_{0}$ (resp. $I_{1} ; \operatorname{resp} . I_{k}$ où $k=2, \ldots, n$ ) $I_{\text {'ensemble des indices } i \in I}$ tels que $a_{i}=0$ (resp. $a_{i}$ fini non nul; resp. $\left.a_{i}=e_{k}\right)$. On obtient ainsi la partition $\left(I_{k}\right)[k=0,1, \ldots, n]$ de $I$.

Il est clair qu'une famille $\left(x_{i}\right)[i \in I]$ est de type $t=\left(a_{i}\right)$ si et seulement si :

$$
\begin{aligned}
& \forall J \subset I_{1}, \operatorname{dim} \varphi\left(x_{J}\right) \geq a_{J} \\
& \text { et } \quad \forall i \in I, a_{i} \text { infini, dim } \varphi\left(x_{i}\right) \geq a_{i} \\
& \text { ces conditions étant bien entendu indépendantes (voir début de } 4.3 \text { ). }
\end{aligned}
$$

Soit $\left(x_{i}\right)$ une famille de type of. Pour $k=0,1, \ldots, n$ on note $J_{k}=U I_{r}[r \leqslant k]$. $\left(X_{i}\right)\left[i \in J_{q}\right]$ contient une famille disjointe $\left(Y_{i}\right)\left[i \in J_{i}\right]$ telle que $r\left(Y_{i}\right)=a_{i}$. Dans $E$ considéré comme $\psi^{1}$-espace où $\psi^{1}=\varphi_{A}, r^{1}=r_{A}$ et $A=U Y_{i}\left[j \in J_{1}\right]$, comme $r(A)$ est finie, pour $k=2, \ldots, n$ et $i \in I_{k}$, 
$r^{1}\left(x_{i}\right) \geq a_{i}$ (c.f. 3.3. corollaire 1 du théorème 3 par exemple). Il va âtre démontré ci-dessous (théorème 2) qu'il existe une famille $\psi^{1}$-disjointe $\left(z_{i}\right)\left[i \in I_{2}\right]$ incluse dans $\left(x_{i}\right) \quad\left[i \in I_{2}\right]$ telle que $r^{1}\left(z_{i}\right)=\ell_{2}$ pour tout $i$. En prenant pour tout $i$ dans $I_{2}$ une $\psi^{1}$-base $Y_{i}$ de $\varphi^{1}\left(z_{i}\right)$ incluse dans $z_{i}$ on obtient une famille $\left(\mathrm{Y}_{\mathrm{i}}\right) \quad\left[i \in \mathrm{I}_{2}\right] \quad \psi$-disjointe telle que $\mathrm{Y}_{\mathrm{I}_{2}}$ et $\mathrm{Y}_{\mathrm{J}_{1}}$ sont $\varphi$-disjoints, donc (associativité) $\left(\mathrm{Y}_{i}\right)\left[i \in \mathrm{J}_{2}\right]$ est $\varphi$-disjointe. On itère de même pour $\mathrm{I}_{3}$ en raisonnant dans $E$ considéré comme $\psi^{2}$-espace sachant que $\psi^{2}=\Psi_{B}$, $B=U Y_{i}\left[i \in J_{2}\right]$ et $r(B)=l_{2}<l_{3}$. Ayant itérer $n-2$ fois ce procédé on obtient $\left(\mathrm{Y}_{i}\right) \quad(i \in \mathrm{I}) \varphi$-disjointe, de type of et incluse dans $\left(\mathrm{x}_{i}\right)[i \in I]$. En particulier on a démontré le résultat suivant qui précise le théorème 1 :

Corollaire 2.- Soit $\mathscr{b}=\left(a_{i}\right)[i \in I]$ une famille finie de cardinaux. Pour qu'une

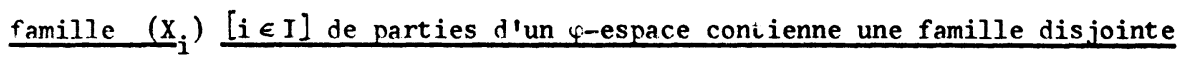
$\left(Y_{i}\right)[i \in I]$ telle que $\operatorname{dim}\left(Y_{i}\right)=a_{i}$ pour tout $i$, il faut et il suffit que :

\begin{tabular}{|l|}
\hline Pour tout $J \subset I^{f}, \quad r\left(x_{J}\right) \geq a_{J}$ \\
\hline Pour tout $i$ tel que $a_{i}$ soit infini, $r\left(x_{i}\right) \geq a_{i}$ \\
\hline
\end{tabular}

ces inégalités étant indépendantes.

I] reste à démontrer le :

Théorème 2.- Soit a un cardinal infini et $I$ un ensemble fini. Pour qu'une fami]le $\left(X_{i}\right) \quad[i \in I]$ de parties d'un $\varphi$-espace contienne une famille $\varphi$-disjointe $\left(Y_{i}\right)[i \in I]$ telle que $\operatorname{dim} \varphi\left(Y_{i}\right)=a$ pour tout $i$, il faut et il suffit que : pour tout $i$

Voici d'abord un lemme qui généralise aux $\varphi$-espaces une propriété classique des parties d'un ensemble. 
Lemme 1.- Soit a un cardinal infini, $M$ et $N$ deux parties d'un $\varphi$-espace $E$ telles que $M U N$ soit $\varphi-l i b r e, \operatorname{card} M=\operatorname{card} N=a$ et $\operatorname{card} M \cap N<a$. Pour toute partie $\underline{X}$ de $E$ telle que $\operatorname{dim} \varphi(X)=a_{2}$ on a la relation :

$$
r_{M}(x)+r_{N}(x)=a
$$

Démonstration du lemme 1

Soit $Y$ une $\varphi_{M}$-base de $X$. D'après le théorème 2 de 2.1 .4$. , on a : $\operatorname{Card} Y=r_{M}(X) \leq r(X)=a$

Si Card $Y=a$, la relation (2) est vérifiée. Sinon on a Card $Y<a$, donc :

$$
\begin{aligned}
& \mathbf{r}_{\mathbf{Y}}(\mathrm{M})=\mathbf{r}_{\mathrm{Y}}(\mathrm{N})=\mathbf{r}_{\mathbf{Y}}(\mathrm{X})=\mathbf{a} \\
& \text { Soit alors } M_{\mathcal{Y}} \text { une } \varphi_{Y}^{\text {-base de } M} \\
& \text { et } \mathrm{N}_{1} \text { une }{ }^{\varphi} \mathrm{Y} \cup \mathrm{M}_{1} \text {-base de } \mathrm{N} \\
& M_{1} \cap N_{1}=\varnothing \text { et } M_{1} \cup N_{1} \text { est } \varphi_{Y} \text {-libre (voir 3.2.), donc } X \text { et } N_{1} \text { sont } \\
& { }^{\varphi} \mathrm{Y}^{\text {-disjointes }}\left(\mathrm{X} \in \varphi_{\mathrm{Y}}\left(M_{\mathcal{Q}}\right)\right) \text { et on a : } \\
& \mathbf{r}_{\mathrm{Y} \cup \mathrm{N}_{1}}(\mathrm{X})=\mathbf{r}_{\mathrm{Y}}(\mathrm{X})=\mathrm{a} \\
& \text { or } Y \text { est une }{ }^{\varphi}{ }_{M \cup N_{1}} \text {-base de } X \text {, et comme } N^{\prime}=N-M n M \text { est la } \\
& \varphi_{M} \text {-base de } N, N_{1} \subset N^{\prime}, N^{\prime}-N_{1} \text { est } \varphi_{M \cup N_{1}} \text {-libre, et comme } \\
& \varphi_{M \cup N_{1}}\left(N^{\prime}-N_{1}\right)=\varphi(M \cup N) \subset \varphi_{M \cup N_{1}}(X) \text {, } \\
& \operatorname{Card}\left(\mathrm{N}^{\prime}-\mathrm{N}_{1}\right) \leq \operatorname{Card} \mathrm{Y}<\mathrm{a} \\
& \text { or Card }(M \cap N)<a \text {, donc : Card }\left(N-N_{1}\right)<a \\
& \text { et } \quad r_{Y \cup N_{1}}(X)=a=r_{Y \cup N}(X) \leq r_{N}(X) \\
& \text { d'où } \quad r_{N}(X)=a
\end{aligned}
$$


Demonstration du théorème 2.

La condition est évidenment nécessaire. Inversement, a étant un cardinal infini, l'on démontre :

$\left(R_{a}\right)\left\{\begin{array}{l}\left(Y_{i}\right)_{i \in I} \text { étant une famille finie telle que } r\left(Y_{i}\right) \geq a \text { pour tout } i \text {, on a : } \\ \forall i \in I, \exists T \in Y_{i} \text { telle que }: r(T)=a \text { et } \forall j \in I, r_{T}\left(Y_{j}\right)=r\left(Y_{j}\right)\end{array}\right.$

In effet soit $i \in I, z_{i}$ une $\varphi$-base de $Y_{i}$ et $n=$ Card $I$. Comme Card $z_{i} \geqslant a$, il existe une famille $\left(T_{m}\right)_{m=1, \ldots, n}$ de parties disjointes deux $a$ deux de $z_{i}$ telles que Card $T_{m}=a$ pour tout $m$. D'après le lemme 1 , pour tout $j \in I$, il existe au plus un $m_{j}$ tel que $r_{T_{m}}\left(Y_{j}\right)<a$. On peut donc choisir $T$ parmi les $T_{\mathbf{m}}$.

En appliquant $\left(R_{a}\right)$ Card I-fois et en ne considérant que des parties libres, on construit aisément une famille $\left(Y_{i}\right)$ disjointe, incluse dans $\left(X_{i}\right)$ et telle que $r\left(Y_{i}\right)=a$ pour tout $i$.

\section{6.- Non-extension de ces résultats aux $\mathscr{L}$-espaces}

L'on peut essayer d'étendre le théorème 1 aux $\mathscr{L}$-espaces. Déjà M.Rado dans [9] a démontré que les axiomes imposés à sa structure sont nécessaires et suffisants pour la validité du théorème 1. Voici le résultat général :

Théorème 3 (Rado).- Soit $E$ un $\mathscr{L}$-espace pour que toute famille de type $A_{f}$ (pour le rang $r_{\mathcal{L}}$ ) contienne une famille $\mathscr{L}$-disjointe de type $A$, il faut et il suffit que $(E, \mathscr{L})$ soit un $\mathscr{L}$-espace régulier, i.e. un $\varphi$-espace.

\section{Démonstration :}

La condition suffisante résulte du théorème 1 . 
La condition est nécessaire car si $(E, \mathscr{L})$ n'est pas régulier :

$$
\exists x \in \mathscr{L} \quad, \quad \exists y \in \mathscr{L} \text { tels que Card } \mathrm{X}=\operatorname{Card} \mathrm{Y}+1=\mathrm{n}
$$

et $\forall \mathbf{X} \in \mathbf{X}-\mathbf{Y} \quad, \quad \mathbf{Y}+\mathbf{x} \notin \mathscr{L}$

Alors $(X, Y)$ est de type $(1, n-1)$, mais $(X, Y)$ ne contient aucune famille disjointe, et de type $(1, n-1)$. 


\section{Chap. V Existence de familles disjointes incluses dans \\ des familles infinies de parties d'un $\varphi$-espace.}

Suivant en cela Bourbaki [C, I, 3], l'on admet comme seuls axiomes logiques l'axiome du choix (sous sa forme usuelle) et l'axiome de l'infini. Sauf mention expresse dans 1 'énoncé du théorème, les résultats énoncés sont indépendants de l'hypothèse du continu généralisé érigé en axiome de la théorie des ensembles par J.P. Cohen (voir par exemple [16] et [17]).

L'on utilisera en 5.2. des raisonnements sur les limites projectives dont certaines parties sont analogues à ceux qui sont exposés dans Bourbaki $[\mathrm{C}, \mathrm{I}, 3], \S 6$, exercice 29 et $[\mathrm{C}, \mathrm{III}, 1]$, Appendice, page 137 de la troisième édition.

Une étude systématique des différents cas possibles pour une famille dénombrable et le principe de l'étude générale de l'une d'elles terminent ce chapitre.

\section{1.- Généralités}

$$
\begin{aligned}
& \text { Soit } A=\left(a_{i}\right)_{i \in I} \quad \text { une famille de cardinaux. Alors } \\
& L=\left\{a_{i} \mid i \in I\right\}=I_{f t} \quad, \quad L=\left\{a_{i} \mid, i \in I \text { et } a_{i} \text { infini }\right\}=I_{A b}^{\omega}
\end{aligned}
$$

est un ensemble bien ordonné par l'ordre naturel des cardinaux et pour tout $l \in L$, l'on pose :

$$
I_{l}=\left\{i \mid i \in I \quad \text { et } \quad a_{i}=\ell\right\} \quad=I_{l}^{i t}
$$

$\left(I_{l}\right)_{\ell \in I}$ est une partition de $I$, et $I^{\prime}$ on pose $I_{l}=\varnothing$ si $l$ est un cardinal qui n'est pas dans $L$. On écrit en outre : 


$$
\begin{aligned}
& I^{f}=\left\{i \mid i \in I \text { et } a_{i} \text { fini non nul }\right\}=I_{d t}^{f}, d^{f}=\left(a_{i}\right)_{i \in I^{f}} \\
& I^{\omega}=\left\{i \mid i \in I \text { et } a_{i} \text { infini }\right\}=I_{d t}^{\omega}, t^{\omega}=\left(a_{i}\right)_{i \in I^{\omega}} \\
& I^{0}=I_{0}=I_{i t}^{0} \\
& \text { et } a_{t b}=\operatorname{Min} a_{i}\left[i \in I \text { et } a_{i} \text { infini }\right]
\end{aligned}
$$

Ces notations seront utilisées sans rappels, en précisant fó quand ce sera nécessaire.

Contrairement au cas fini, en général une famille infinie de type to ne contient pas une famille disjointe de type of . Voici deux exemples typiques :

Exemple 1.- Soit of telle que Card $I^{f} \geq a$ of . E étant un ensemble tel que Card $E={ }^{a}$ et $\left(E_{i}\right)_{i \in I}$ une partition (sens large) de $E$ telle que pour tout $i \in I$ Card $E_{i}=a_{i}$, considérons la famille $\left(X_{i}\right)_{i \in I}$ définie par

$$
\begin{array}{ll}
\forall i \in I_{a_{\theta}}, & x_{i}=F \quad \text { où } F \subset E_{I^{f}} \text { et } \quad \text { Card } F=a a_{b} \\
\forall i \in I-I_{a_{A b}}, & x_{i}=E_{i}
\end{array}
$$

E étant $\varphi$-espace discret, $\left(x_{i}\right)_{i \in I}$ est de type of et ne peut contenir aucune famille disjointe de type it.

Exemple 2.- Soit to une famille telle que pour un cardinal infini $l \in L$ on ait : Card $I_{\ell}>\ell$. E étant un ensemble tel que Card $E=a_{I}$ et $\left(E_{i}\right)_{i \in I}$ une partition (au sens large) de $E$ telle que pour tout $i \in I, \operatorname{Card} E_{i}=a_{i}$, prenons $X \subset E$ telle que Card $X=l, Y \subset E$ telle que Card $Y=$ Card $I_{\ell}$,

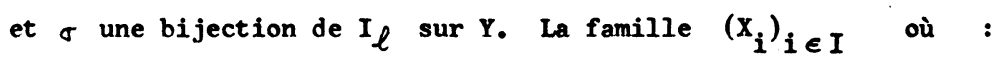

$$
\begin{aligned}
& \forall i \in I_{l}, \quad x_{i}=x \quad u \quad\{\sigma(i)\} \\
& \forall i \in I-I_{l}, x_{i}=E
\end{aligned}
$$


est de type $\mathcal{f}_{\hat{f}}$ dans le $\varphi$-espace discret $E$, mais né contient aucune famille disjointe de type $t$.

On peut donc particulariser les familles $\mathbb{t}$ de la manière suivante' :

Définition 1.- Une famille $t=\left(a_{i}\right)_{i \in I}$ est dite lisse si elle vérifie la propriété :

$$
\forall l \in \mathrm{L}^{\omega} \text {, Card } \mathrm{I}_{\ell}<\ell \text { et } \sum_{0<\mathrm{m}<\ell} \text { Card } \mathrm{I}_{\mathrm{m}}<\ell
$$

t est dite faiblement lisse si

(LISf) Card $\mathrm{I}^{\mathrm{f}}<a_{t}$ et $\forall l \in \mathrm{L}^{\omega}$, Card $\mathrm{I}_{\ell} \leq \ell$

to est dite rugueuse si elle n'est pas faiblement libre.

Si thest faiblement lisse, pour tout $\mathrm{J} \subset \mathrm{I}$, si $\mathrm{J} \cap \mathrm{I}^{\omega} \neq \varnothing$, $a_{J}=\operatorname{Sup} a_{i}[i \in J]$. Donc pour tout $l \in \mathbb{L}^{\omega}$ :

$\sum_{0 \leqslant m \leqslant l}$ Card $I_{m} \leqslant \ell$

Toute famille lisse est donc faiblement lisse

Si $\left(x_{i}\right)$ est de type $A$, on a :

$\left(\forall i \in I \omega, r\left(x_{i}\right) \geq a_{i}\right)$ et $\left(\forall J \in \mathcal{F}^{\prime}\left(I_{d}^{f}\right), \quad r\left(x_{J}\right) \geq a_{J}\right)$

inversement, it étant fixée, toute famille $\left(x_{i}\right)$ vérifiant (1) est de type $f_{\text {f }}$ si et seulement si :

$$
\forall \mathrm{J} \subset \mathrm{I}^{\omega} \quad, \quad \mathrm{a}_{\mathrm{J}}=\sup a_{i} \quad\left[i \in I^{\omega}\right]
$$

i.e. si et seulement si :

$$
\forall l \in \mathrm{I}_{\mathbb{A}}^{\mathrm{w}}, \quad \text { Card } \mathrm{I}_{\ell}^{\sqrt{6}} \leq \ell
$$

Les exemples 1 et 2 montrent que, en général, une famille de type of rugueuse ne contient pas de famille dis jointe de type to.

Il va être démontré ci-après que : 
Si to est lisse (resp. si to est faiblement lisse et si l'on admet 1 'hypothèse du continu généralisé), et si en outre :

(CF) (*) $\forall X \subset L^{\omega}$ tel que " $Y Y \subset X, \sup Y \in X \cup\{\sup X\} "$, on a $a(X)<\omega_{1}$

et $\operatorname{Card}\left\{\ell \mid \ell \in I^{\omega}, U_{0}<\bar{l}<\ell\right.$ et $\left.\sup L_{n}^{\omega}\right] \leftarrow, l[=\ell\}<U_{2}$

et $L^{\omega}$ est de caractère final dénombrable au plus, azors toute famillle $\left(x_{i}\right)$ de type to telle que :

$\forall i \in I^{f}, \quad r\left(x_{i}\right)$ soit fini

contient une famille $\varphi$-disjointe de type $t_{b}$.

Le problème consistant à chercher si toute famille de type $A_{\text {f }}$ faiblement lisse contient une famille disjointe de type ô sans utiliser 1 'hypothèse du continu généralisé est laissé ouvert, ainsi que celui qui consiste à généraliser le résultat ci-dessus aux familles ne vérifiant pas la propriété (CF).

En 5.2. l'on étudie les familles of de cardinaux finis, puis en 5.3 . est explicitée la séparation naturelle entre cardinaux finis et cardinaux infinis. Les familles de cardinaux infinis font l'objet de 5.4. et en 5.5. une discussion générale des familles dénombrables termine ce chapitre.

\section{2.- Familles to de cardinaux finis}

Il est deux cas où l'on peut conclurè à l'existence d'une famille disjointe incluse de type $\mathscr{A}_{\mathrm{b}}$ :

- celui où $r\left(x_{i}\right)$ est fini pour tout $i$

- celui où $r\left(x_{i}\right)$ est infini pour tout $i$ sauf un nombre fini et où les $a_{i}$ non nuls sont égaux à 1 .

5.2.1. - Cas où les $x_{i}$ sont tous de rang fini.

L'on va démontrer le :

(*) si $a=U_{\alpha}$, l'on désigne par $\omega_{\bar{\alpha}}$ le caractère final de $\omega_{\alpha}$ et $\bar{a}=U_{\bar{\alpha}}$. 
Théorème 1.- Une famille $\left(x_{i}\right)_{i \in I}$ telle que $r\left(x_{i}\right)$ soit fini pour tout $i$, est de type $t=\left(a_{i}\right)$ si et seulement si :

$$
\forall J \in \dot{H}^{\prime}(I) \quad, \quad r\left(x_{J}\right) \geqslant a_{J}
$$

et alors elle contient une famille $\varphi$-disjointe de type $t$.

\section{a) Démonstration par limite projective}

Soit $\left(x_{i}\right)_{i \in I}$ de type of $=\left(a_{i}\right)$ où $r\left(x_{i}\right)$ est fini pour tout $i$. Prenons alors pour chaque $i \in I$ une base $z_{i}$ de $x_{i}$ incluse dans $x_{i},\left(z_{i}\right)$ est de type $t,\left(z_{i}\right) \subset\left(x_{i}\right)$ et $z_{i}$ est fini pour tout $i$.

Considérons alors pour toute; partie $\mathrm{J}$ de $I$ I'ensemble $G_{J}$ des familles $\left(Y_{i}\right)$ incluses dans $\left(z_{i}\right)$, disjointes et telles que $r\left(Y_{i}\right)=$ Card $\left(Y_{i}\right)=a_{i}$ pour tout 1 .

Si $\mathrm{J} \subset \mathrm{K} \in \mathrm{I}$

$f_{J K}:\left(Y_{i}\right)_{i \in K} \longrightarrow\left(Y_{i}\right)_{i \in J}$

est une application de $G_{K}$ dans $G_{J}$.

$\mathrm{n}$ est clair que $\left(\mathrm{G}_{\mathrm{J}}, \mathrm{f}_{\mathrm{JK}}\right) \quad\left[\mathrm{J}, \mathrm{K} \in \mathcal{F}_{(\mathrm{I})}\right) \quad$ est un système projectif d'ensembles et que :

$$
G_{I}=\underline{l i m} \quad\left(G_{J}, f_{J K}\right) \quad\left[J, K \in F^{\prime}(I)\right]
$$

Or d'après (4) pour tout $J \in \mathcal{F}(I), G_{J}$ est fini et non vide,

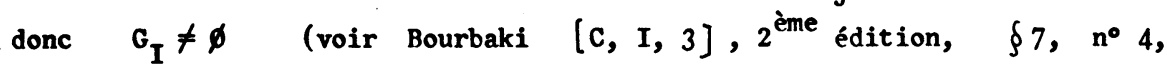
Théorème 1 et exemple 1.)

Voici un raisonnement élémentaire qui permet de démontrer que $G_{I} \neq \varnothing$. 
10) L'on restreint d'abord les $G_{J}$ de façon à rendre les restrictions des $\mathbf{f}_{\mathrm{JK}}$ surjectives :

$$
\begin{aligned}
& \forall \mathrm{J} \in \mathcal{G}(\mathrm{I}) \quad, \text { soit } \quad \mathbf{G}_{\mathbf{J}}^{0}=\mathrm{G}_{\mathbf{J}} \\
& G_{J}^{1}=\cap f_{J K} \quad\left(G_{K}^{0}\right) \quad\left[K \in \mathcal{J}^{*}(I) \text { et } K \supset J\right] \\
& G_{J}^{n}=n \quad f_{J K} \quad\left(G_{K}^{n-1}\right)\left[K \in F^{(I)} \text { et } K \supset J\right]
\end{aligned}
$$

d'où la suite indéfiniment décroissante :

$$
\forall J \in G(I) \quad, \quad G_{J}=G_{J}^{0} \supset G_{J}^{1} \supset \ldots \supset G_{J}^{n-1} \supset G_{J}^{n} \supset \ldots
$$

on démontre aisément par récurrence sur $n \geqslant 0$ que :

$$
G_{J}^{n} \neq \varnothing \quad \text { pour tout } J \in \mathcal{F}^{\prime}(I)
$$

$\mathrm{G}_{\mathrm{J}}$ étant fini, la suite est stationnaire et

$$
\mathrm{F}_{\mathrm{J}}=\bigcap_{\mathrm{n}} \mathrm{G}_{\mathrm{J}}^{\mathrm{n}} \neq \varnothing
$$

et la restriction $g_{J K}$ de $f_{J K}$ à $F_{J}$ est une surjection de $F_{J}$ sur $F_{K}$ telle que :

$$
\underline{\lim }\left(\mathrm{c}_{\mathrm{J}}, \mathrm{f}_{\mathrm{JK}}\right)=\underset{\lim }{\longleftarrow}\left(\mathrm{F}_{\mathrm{J}}, \mathrm{g}_{\mathrm{JK}}\right)
$$

Le fait que les $g_{J K}$ sont surjectives est insuffisant pour démontrer que la limite projective n'est pas vide (voir $[C, I, 3], \S 1$, exercice 32).

$2^{\circ}$ ) En utilisant le théorème de Zorn, l'on démontre $G_{I} \neq \emptyset$. En effet $\mathcal{E}$ l'ensemble des familles $\left(Y_{i}\right)_{i \in K}$ telles que $K \subset I$ et pour tout $J \in \mathcal{F}(K)$, $\left(\mathrm{Y}_{\mathbf{i}}\right)_{\mathrm{i} \in \mathrm{J}} \in \mathrm{F}_{\mathrm{J}} \quad \mathcal{E}$ ordonné par la relation :

$$
\left(\left(Y_{i}\right)_{i \in K} \leq\left(z_{j}\right)_{j \in L}\right) \Longleftrightarrow\left(K \subset L \quad \text { et } \forall i \in K, \quad Y_{i}=z_{i}\right)
$$


est inductif. On démontre alors aisément que tout élément maximal de $\mathcal{E}$ est un élément de $C_{I}$.

\section{b) Démonstration par suppressibilité.}

Comme en a) l'on raisonne sur $\left(z_{i}\right)$. L'on va d'abord démontrer que : $\left(R_{A}\right)\left[\right.$ Pour tout $i \in I$ tel que $\operatorname{Card} z_{i}>a_{i}$, il existe un $\dot{x}$ dans $z_{i}$ suppressible, i.e. tel que la famille $\left(T_{j}\right)_{j \in I}$, où $T_{j}=z_{j}$ si $j \neq i$ et $T_{i}=z_{i}-x$, vérifie :

$$
\forall \mathrm{J} \in \mathscr{F} \quad(\mathrm{I}) \quad, \quad \mathrm{r}\left(\mathrm{T}_{\mathrm{J}}\right) \geq \mathrm{a}_{\mathrm{J}}
$$

Soit en effet $i \in I$ tel que Card $z_{i}>a_{i}$.

$x \in z_{i}$ est suppressible si et seulement si :

$$
\forall J \in F_{(I-i)}, r\left(z_{J} \cup\left(z_{i}-x\right)\right) \geq a_{J}+a_{i}
$$

i.e. si et seulement si pour tout $J \in \mathcal{F}(I-i), x$ est suppressible dans $\left(z_{j}\right)_{j \in J+i}$

$$
\text { Or } z_{i} \text { est fini et } s i x \in z_{i} \text { n'est pas suppressible, il existe }
$$

$J_{x} \in \mathcal{F}(I-i)$ tel que $x$ ne soit pas suppressible dans $\left(z_{j}\right)_{j \in J_{x}}+i$. Posant $K=J_{z}+i$, à fortiori tout $x \in z_{i}$ n'est pas suppressible dans $\left(z_{j}\right)_{j \in K}$, ce qui contredit le fait que $\left(z_{j}\right)_{j \in K}$ soit de type $\mathscr{A}_{K} \cdot D^{\prime}$ 'où $\left(\mathbb{R} \mathbb{A}_{6}\right)$.

Considérons maintenant un bon ordre sur I (si I est dénombrable, on ordonne I suivant le type d'ordre $\omega_{0}$ et l'on raisonne par récurrence infinie classique). L'on construit par récurrence transfinie sur $i$ une famille $\left(Y_{i}\right)_{i \in I}$ telle que Card $Y_{i}=a_{i}$ pour tout $i \in I$ et telle que :

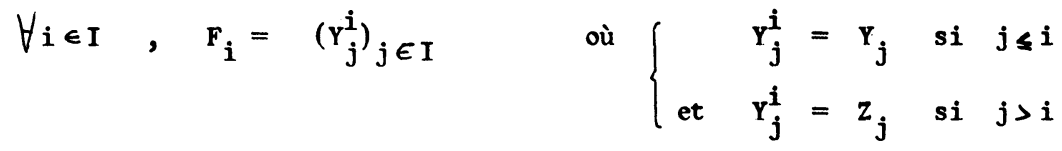


vérifie (4'), en appliquant $\left(R_{A}\right)$.

Alors $\left(Y_{i}\right)_{i \in I}$ vérifie aussi (4'), et comme Card $Y_{i}=a_{i}$ pour tout $i \in I, \quad\left(\mathrm{Y}_{i}\right)_{i \in I}$ est disjointe (voir 3.3. théorème 4).

c) Indépendance des conditions (3).

Les conditions $r\left(x_{J}\right) \geqslant a_{J}$ quand $J \in F^{(I)}$ sont indépendantes si
les $a_{i}$ sont $>0:$

Soit en effet $\left(\mathrm{Y}_{i}\right)_{i \in I}$ une famille disjointe de parties libres telles que Card $Y_{i}=a_{i}$ pour tout $i$, et soit $J \in \mathcal{F}(I)$. Si l'on prend $x \in Y_{J}$ et si 1 'on considère

$$
\begin{aligned}
\mathrm{x}_{\mathrm{i}} & =\mathrm{Y}_{\mathrm{J}}-\mathrm{x} & \text { si } & i \in J \\
\text { et } \quad \mathrm{x}_{\mathrm{I}} & =\mathrm{Y}_{\mathrm{J}} \cup \mathrm{Y}_{\mathrm{i}} & \text { si } & i \in \mathrm{I}-\mathrm{J}
\end{aligned}
$$

l'on obtient une famille $\left(x_{i}\right)_{i \in I}$ qui vérifie $r\left(x_{K} \sum^{a}{ }_{K}\right.$ pour tout $K \in J^{\prime}(I)$ sauf pour $K=J$.

5.2.2.- Cas où les $\mathrm{x}_{\mathrm{i}}$ sont presque tous de rang infini.

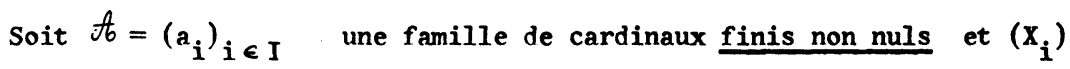
une famille de parties d'un $\varphi$-espace $E$. Alors $B=\left(b_{i}\right)$ où $b_{i}=r\left(x_{i}\right)$ pour tout $i$ est telle que :

pour $K=I_{\beta}^{f}, \quad\left(X_{i}\right)_{i \in K} \quad$ contienne $\quad\left(Y_{i}\right)_{i \in K} \quad \varphi$-disjointe, de type $A^{K}$, vérifiant $r\left(Y_{K}\right)=a_{K}$.

$$
\begin{aligned}
& \text { Si } K \text { est fini, } \quad r\left(Y_{K}\right)=a_{K} \text { est fini } \\
& \text { Si } K \text { est infini, } r\left(Y_{K}\right)=a_{K}=\text { Card } K \text {, et alors si en outre }
\end{aligned}
$$

Card $K \geq a, B$, l'on a vu en 5.1. exemple 1 que $\left(X_{i}\right)_{i \in I \text { ne contient pas er }}$ général de famille disjointe de type to. 
Par contre si Card $K<a_{\beta}$, considérant $\mathrm{E}$ comme $\Psi$-espace où $\Psi=\varphi_{Y_{K}}$, pour tout $i \in I_{\beta}^{\omega}, r_{\varphi}\left(x_{i}\right)=r_{\Psi}\left(x_{i}\right)=t_{i} \quad$ est infini, et alors il est raisonnable d'emettre la conjecture suivante :

Soient $\mathscr{A}=\left(a_{i}\right)$ une famille de cardinaux finis non nuls et $\left(x_{i}\right)$ une famille de parties d'un $\varphi$-espace, ayant toutes un rang infini. Comme pour toute partie infinie $\mathrm{J}$ de $\mathrm{I}, \mathrm{a}_{\mathrm{J}}=$ Card $\mathrm{J}$ :

$$
\left(x_{i}\right) \text { est de type } \mathscr{A} \text { si et seulement si }\left(x_{i}\right) \text { est de type } 1
$$

et dans ce cas $\left(x_{i}\right)$ contient une famille disjointe de type $\mathscr{C}$ pour toute famille $\mathscr{C}=\left(c_{i}\right)$ de cardinaux finis non nuls telle que :

$$
\sum\left(c_{i}-1\right) \leq \operatorname{Min}\left(r\left(x_{i}\right)\right)
$$

Voici un exemple de familles $\mathscr{C}$ et $\left(x_{i}\right)$ de type 1 ne vérifiant pas (5) et telle que $\left(x_{i}\right)$ ne puisse contenir de famille disjointe de type $\mathscr{C}:$

exemnle 3 : Soit $\mathscr{C}=\left(c_{i}\right)_{i \in I}$ une famille infinie de cardinaux finis non nuls telle que $c=\Sigma\left(c_{i}-1\right)$ soit infini et $>u_{0}$. Soit alors $E$ un ensemble de cardinal celui de $I, \sigma$ une bijection de $I$ sur $E$ et $X \subset E$ telle que Card $\mathrm{X}<\mathrm{c}$ et Card $\mathrm{X}$ infini

$$
\text { Posant pour tout } i \in I, \quad x_{i}=\sigma(i) \cup x, \quad\left(x_{i}\right) \text { est une famille de }
$$

type 1 et $\mathscr{C}$ qui ne peut contenir de famille disjointe de type $\mathscr{C}$.

(*) Cn identifie le cardinal a et la famille $\mathscr{t}=\left(a_{i}\right)$ telle que $a_{i}=a$ pour tout i. 
5.3.- Réduction de l'étude d'une famille $\left(X_{i}\right)_{i \in I}$ quelconque.

Quand on se donne a priori une famille $\left(x_{i}\right)_{i \in I}$ de parties d'un $\varphi$-espace $E$, on peut chercher quel est $l^{\prime}$ ensemble $\mathcal{H}^{\prime}$ des familles $t b^{\prime}=\left(a_{i}\right)$ telles que $\left(x_{i}\right)$ soit de type $t$, ou mieux quel est $l$ 'ensemble $\mathcal{H}$ des familles $t_{t}=\left(a_{i}\right)$ telles que $\left(x_{i}\right)$ contienne une famille $\varphi$-disjointe $\left(Y_{i}\right)$ de type of.

Soit $\mathcal{Y}$ l'ensemble des familles $\left(Y_{i}\right) \varphi$-disjointes, incluses dans $\left(X_{i}\right)$. $\mathscr{Y}$ est inductive, donc admet des éléments maximaux et $\left(\mathrm{Y}_{\mathbf{i}}\right) \rightarrow\left(\operatorname{dim} \varphi\left(\mathrm{Y}_{\mathbf{i}}\right)\right)$ est une application de $\varphi$ sur $\mathscr{H}$. Cependant $\mathscr{H}$ n'est pas inductive en général.

Exemple - Soit $E$-espace discret de cardinal infini a et $\left(x_{i}\right)_{i \in I}$ famille de parties de $E$ telle que : card $I>a$ et $x_{i}=E$ pour tout $i \in I$. Il est clair que Hentient toutes les familles $A^{J} J=\left(a_{i}\right)_{i \in I}$ où $a_{i} J=0 \quad$ si $i \notin J, a_{i} J=a$ si $i \in J$ et card $J=a$; mais si $a^{+}$est le successeur de a et si card $\mathrm{J}=\mathrm{a}^{+}, A^{\mathrm{J}} \notin \mathscr{H}$, bien que borne supérieure d'éléments de $\mathscr{H}$ (Notons que ceci peut se produire également quand $\left(\operatorname{dim} \varphi\left(x_{i}\right)\right)_{i \in I}$ est lisse).

\subsection{1.- Si $r\left(X_{i}\right)$ est fini pour tout $i \in I$.}

En convenant que $B=\left(b_{i}\right)_{i} \in I_{1}$ est fini si et seulement si $b_{i}=0$ pour tout $i$ sauf un nombre fini, i.e. si et seulement si $b_{I}<s^{s}$, et en désignant par $\mathscr{f}(\mathbb{f}) \quad l$ 'ensemble des "parties finies" de $\mathscr{A}$, i.e. des $B=\left(b_{i}\right) \leq A=\left(a_{i}\right)$ telles que $b_{i}=0$ pour tout $i \in I$ sauf un nombre fini d'indices $i$ pour lesquels $b_{i}=a_{i}$, alors il résulte du corollaire du théorème 1 que :

$\mathcal{H}$ est de caractère fini et $\quad \underline{H}=H_{1}$

Donc : $\quad$ est inductif et admet des éléments maximaux.

On démontre alors que :

- toute of $\in H_{e s t}$ inférieure ou égale $3 \quad\left(r\left(x_{i}\right)\right)_{i \in I}$

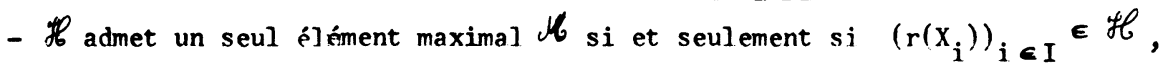


i.e. si et seulement si $\left(x_{i}\right)$ est $\varphi$-disjointe; et dans ce cas $d f=\left(r\left(x_{i}\right)\right)$

- pour une $\mathscr{t} \in \mathscr{H}$, les propriétés suivantes sont équivalentes :

a) - to est maximale dans $H_{\ell}$

b) - Il existe une $\left(Y_{i}\right) \varphi$-disjointe, incluse dans $\left(X_{i}\right)$ et telle que $\operatorname{dim} \varphi\left(Y_{i}\right)=a_{i}$ pour tout $i \in I$ et $Y_{I} \varphi$-engendre $\varphi\left(X_{I}\right)$.

En outre dans ce cas, toute $\left(z_{i}\right) \varphi$-disjointe, incluse dans $\left(x_{i}\right)$ et de type fo est telle que $z_{I} \varphi$-engendre $\varphi\left(X_{I}\right)$ et $\operatorname{dim} \varphi\left(z_{i}\right)=$ a $_{i}$ pour tout $i$; en particulier $a_{I}=\operatorname{dim} \varphi\left(x_{I}\right)$.

Les constructions faites en 4.2 . et 5.2 . permettent alors de construire $\mathscr{Y}$, d'où $\mathscr{H}=H_{\mathcal{C}}^{\prime} \quad$ (il suffit d'en connaître les éléments maximaux).

\section{$\underline{5.3 .2 .- \text { Si pour certains } i \in I, r\left(X_{i}\right) \text { est infini }}$}

Soit $B=\left(\mathbf{r}\left(\mathbf{x}_{\mathbf{i}}\right)\right) \quad$ et $\quad I^{0}=I_{\beta}^{0} \ldots$.

On peut négliger systématiquement $\left(\mathrm{X}_{i}\right)_{i \in \mathrm{I}^{\circ}}$, de sorte que l'on
suppose $\mathrm{I}^{\circ}=\varnothing$ par la suite.

On résoud $\left(x_{i}\right)_{i \in I^{f}}$ comme dans 5.3.1. d'où $\mathscr{H}_{f}$ et $\mathcal{S}_{f}$. On indiquera ci-après comment résoudre $\left(x_{i}\right)_{i \in I} \omega$, d'où $H_{\omega} \subset \mathscr{W}_{\omega}^{\prime}$ et $\mathcal{J}_{\omega}$. Alors deux cas se présentent :

a) - ou bien $\forall i \in I^{\omega}, \quad r\left(x_{I} f^{\prime}<r\left(X_{i}\right)\right.$ et alors :

$$
\text { et } \begin{aligned}
& \varphi \rightleftharpoons \varphi_{f}+\varphi_{w} \\
& H=H_{f}+H_{\omega}
\end{aligned}
$$

où ces notations ont le sens suivant :

$$
\forall\left(Y_{i}\right)_{i \in I} \in \mathcal{Y}, \quad\left(y_{i}\right)_{i \in I^{f}} \in \rho_{f} \quad \text { et } \quad\left(Y_{i}\right)_{i \in I} \in \in \rho_{\omega} \quad \text { d'où } \rightarrow \text {. }
$$


Réciproquement,

$$
\forall\left(\mathrm{Y}_{i}\right)_{i \in I^{f}} \in \mathcal{Y}_{f}, \quad \forall\left(\mathrm{Y}_{\mathbf{i}}\right)_{i \in I} \omega \in \mathcal{S}_{\omega} \quad \text { en général }\left(\mathrm{Y}_{i}\right)_{i \in I} \notin \mathcal{Y},
$$

mais on peut modifier $\left(Y_{i}\right)_{i \in I} \omega$ de façon qu'en fin de compte $\left(Y^{\prime}{ }_{i}\right)_{i \in I} \in \mathcal{S}$ où $\quad Y^{\prime}{ }_{i}=Y_{i} \quad \forall i \in I^{f}$. Mieux, on peut le faire de manière que $\operatorname{dim} \varphi\left(Y_{i}\right)=\operatorname{dim} \varphi\left(Y_{i}\right) ;$ signalons cependant que, si certains $\operatorname{dim} \varphi\left(Y_{i}\right)$ pour $i \in I^{\omega}$ sont $<\sum \operatorname{dim} \varphi\left(Y_{i}\right) \quad\left[i \in I^{f}\right]$, il peut être nécessaire de changer complètement ces $Y_{i} \quad$ (sinon il suffit de prendre $Y^{\prime}{ }_{i} \varphi_{Y_{I} f}$-base de $\varphi\left(Y_{I f} \cup Y_{i}\right)$, incluse dans $\left.\mathbf{Y}_{\mathbf{i}}\right)$. D'où la seconde égalité et

b) - ou bien : $\exists i \in I^{\omega}, \operatorname{dim} \varphi\left(x_{I^{f}}\right) \geqslant \operatorname{dim} \varphi\left(x_{i}\right)$

dans ce cas $H_{c} \mathscr{H}_{f}+H_{\omega} \quad l$ 'inclusion étant stricte en général.

Nous verrons sur un exemple (au 5.5. quand I est dénombrable) comment on peut mener cette étude. Signalons qu'il est bon en général de se ramener à a) en restreignant les $x_{i}$ pour $i \in I, \quad\left(o u i \in I^{\omega}\right)$. De toute façon on peut toujours raisonner dans $(E, \Psi)$ où $\psi=\varphi_{X_{I^{f}}}$ et y résoudre $\left(X_{i}\right)_{i \in I} \omega$, en passant aux $\Psi$-bases (qui sont $\varphi$-libres), et en accolant $\left(Y_{i}\right)_{i \in I} \omega \in \mathcal{Y}_{f}$ on a alors une solution pour I. D'où

$$
\varphi_{\leftarrow} \varphi_{1}(\operatorname{pour} \varphi)+\mathscr{S}_{\omega} \quad\left(\text { pour } \varphi_{\mathrm{x}_{\mathrm{I}^{\mathrm{f}}}}\right)
$$

5.3.3.- Réduction de $\left(\mathrm{X}_{\mathrm{i}}\right)$

La relation : $i \propto j$ si et seulement si $\varphi\left(X_{i}\right) \subset \varphi\left(X_{j}\right)$ définit un préordre sur $I$, et si $p$ est la relation d'équivalence canoniquement associée, soit $\mathrm{L}=\mathrm{I} / \mathrm{P},\left(\mathrm{x}_{\ell}\right)_{\ell \in \mathrm{L}}$ oì $\mathrm{x}_{\ell}=\varphi\left(\mathrm{x}_{\mathbf{i}}\right) \forall \mathrm{i} \in \ell$ :

$"\left(x_{i}\right) \quad$ est de type $\left(a_{i}\right) " \Longleftrightarrow "\left(x_{\ell}\right)$ est de type $\left(a_{\ell}\right) "$

où $a_{\ell}=\sum_{i \in \ell} a_{i}$, et on passe facilement de $\varphi_{1} \quad\left(\right.$ pour $\left(x_{l}\right)$ ) à $\varphi$ (pour $\left(x_{i}\right)$ ) et inversement (on "transporte" les partitions de cardinaux finis ou 
infinis représentées par les sommes cardinales).

On peut donc toujours se ramener à $l^{\prime}$ 'étude des familles $\left(x_{i}\right)$ telles que $\varphi\left(x_{i}\right) \neq \varphi\left(x_{j}\right)$ pour tout $i \neq j$. De telles familles sont dites réduites (c'est la généralisation de la notion de réduction vue quand I est fini en 4.4 ).

\section{4.- Familles $t$ de cardinaux infinis}

\subsection{1.- La topologie sur I et le théorème fondamental}

$$
\text { Soit } t=\left(a_{i}\right)_{i \in I} \text { une famille de cardinaux infinis. }
$$

L'application qui ̀̀ toute partie $J$ de $I$ associe :

$$
\bar{J}=\left\{i \mid i \in I \text { et } \exists K<J \text { tel que Sup } a_{j}[j \in J]=a_{i}\right\}
$$

est une fermeture topologique, qui définit la topologie $\varpi_{I}=t_{I}^{t}$ sur $I$. $\pi: \mathrm{i} \longrightarrow \mathrm{a}_{\mathbf{i}}$ est une application de $I$ dans l'ensemble $\mathrm{E}_{a}$ des cardinaux inférieurs ou égaux à $a=a^{t}=\operatorname{Sup~} a_{i}[i \in I]$, qui se restreint au but en une surjection $\pi_{0}$ de I sur L .

Par $\mathscr{L} \doteq(\ell)_{\ell \in L}, I$ est muni comme ci-dessus d'une topologie $\mathscr{q}_{L}^{\mathcal{L}}=q_{L}$, et $\mathscr{G}_{I}$ est l'image réciproque par $\pi_{0}$ de $\mathscr{G}_{L}$ (au sens de $[\mathrm{C}, \mathrm{II}, 1], \S 2,3$, exemple I, p. 30).

De même pour tout cardinal a, $\mathrm{E}_{\mathrm{a}}$ est muni de la topologie $\mathscr{E}_{\mathrm{a}}$, et, si $L \subset E_{a}, \mathscr{G}_{L}$ est induite par $\mathscr{G}_{a}$ sur $L$, et $\mathscr{G}_{I}$ est I'image réciproque par $\pi$ de $b_{a}$ :

$$
\forall \mathrm{J} \subset \mathrm{I}, \quad \overline{\mathrm{J}}=\pi^{-1} \overline{(\pi(\mathrm{J}))}=\pi_{0}^{-1} \frac{\left(\pi_{0}(\mathrm{~J})\right)}{4}
$$

\section{Etude sommaire de la topologie $\varnothing_{a}$}

Une partie $X$ de $E_{a}$ est fermée si et seulement si elle vérifie 1 'une des propriétés équivalentes :
1) $\forall Y \subset X, \quad$ Sup $Y \in X$
2) $\forall \mathrm{b} \in \mathrm{E}_{\mathrm{a}}, \quad \sup \ell[\ell \in \mathrm{x}$ et $\ell<\mathrm{b}] \in \mathrm{x}$ 
or, pour tout $l \in E_{a},\left[U_{0}, l\right]$ et $[\ell, a]$ sont fermés ; donc : $\left.\forall \ell \in \mathrm{E}_{\mathrm{a}}, \forall \mathrm{m} \in \mathrm{E}_{\mathrm{a}}, \ell<\mathrm{m}, \quad\right] \ell, \mathrm{m}[$ est ouvert et $[\ell, m]$ est fermé.

et

$\left.\left.\forall \ell \in E_{a}-a,\left(\left[U_{0}, \ell\right],\right] \ell, a\right]\right)$ est une partition de $E_{a}$ en deux ensembles à la fois ouverts et fermés, donc $\mathscr{E}_{a}$ est totalement discontinue. En particulier $\{\ell\}$ est fermé, mais n'est ouvert que si $l$ a un prédécesseur.

$\dot{\sigma}_{a}$ est compacte : on vérifie qu'elle est séparée. Soit en outre

$\left(x_{i}\right)_{i \in I}$ une famille de fermés de $E_{a}$ telle que :

$\forall \mathrm{J} \in \mathcal{F}(\mathrm{I}), \quad \cap \mathrm{x}_{\mathbf{i}}[\mathrm{i} \in \mathrm{J}] \neq \varnothing$

Alors $a^{J}=\operatorname{Max}\left(\cap x_{i}[i \in J]\right)$ existe pour tout $J \in \mathscr{H}(I)$

et $\quad a=\operatorname{Min} a^{J}[J \in J(I)]$ est atteint pour un $J \in \mathscr{F}(I)$

au moins : $a=a^{J}$. Comme

$$
\forall \mathrm{K} \in \mathcal{F}(\mathrm{I}), \quad \mathrm{K} \supset \mathrm{J}, \quad \mathrm{a} \leqslant \mathrm{a}^{\mathrm{K}} \leqslant \mathrm{a}^{\mathrm{J}}, \quad \mathrm{a}^{\mathrm{K}}=\mathrm{a}^{\mathrm{J}}=\mathrm{a}
$$

donc a $\in \cap x_{i}[i \in I]$ et l'axiome (C") de $[C, I I, 1], \S 9,1$.

Définition.- Soit $A_{b}=\left(a_{i}\right)_{i} \in I$ une famillle de cardinaux et $L^{\omega}$ l'ensemble des cardinaux infinis. On appelle caractère fermé de $t 6$ (resp. de $L^{c}$ ) le plus petit ordinal $\alpha$ tel oue :

pour tout $M C I^{\omega}$ tel que $M \cup\{\operatorname{Sup} M\}$ fermé, on a : $\operatorname{Ord}(M)<\alpha$ et l'on écrit $\underline{C F\left(t_{t}\right)}$ (resp. $C F\left(L^{\omega}\right)$ ) le caractère fermé de to (resp. $L^{\omega}$ ).

On appelle caractère ouvert de to (resp. de $\mathrm{L}^{\omega}$ ), l'ordinal :

$$
\mathrm{C} \cap(\mathrm{t})=\mathrm{C} \cap\left(\mathrm{L}^{\omega}\right)=\text { ord }\left(\mathrm{O}\left(\mathrm{L}^{\omega}\right)\right)
$$

où $\left(^{*}\right): \quad O\left(\mathrm{~L}^{\omega}\right)=\left\{\ell \mid \ell \in \mathrm{L}, U_{0}<\bar{l}<\ell\right.$ et $\operatorname{Sup~} \mathrm{m}[\mathrm{m} \in \mathrm{L}$ et $\left.\mathrm{m}<\ell]=\ell\right\}$

( $^{*}$ Si $a=U_{\alpha}$, J'on désigne par $\omega_{\bar{\alpha}}$ le caractère final de $\omega_{\alpha}$ et $\bar{a}=U_{\alpha}$. 
Soit $\lambda=\operatorname{Sup}(\operatorname{Ord} M)\left[M \subset L^{\omega}\right.$ et $M \cup\{\operatorname{Sup} M\}$ fermé $\}$.

Si la borne supérieure est atteinte, $\operatorname{CF}\left(f_{t}\right)=\lambda+1 ; \operatorname{sinon}, \operatorname{CF}\left(t_{t}\right)=\lambda$.

Si $I^{\omega}$ est fini, $\quad \operatorname{CF}\left(t_{t}\right)=$ Ord $\left(L^{\omega}\right)$

Si $L^{\omega}$ est infini, $\operatorname{Cf}\left(t_{t}\right) \geqslant \omega_{0}$, , mais $\operatorname{CF}\left(t_{t}\right)$ peut être un ordinal infini absolument quelconque $\leqslant \operatorname{Ord}\left(L^{\omega}\right)+1$.

Le but de ce paragraphe est de démontrer le :

Théorème 2.- Si $t=\left(a_{i}\right)$ est une famille lisse (resp. si l'on admet l'hypothèse du continu généralisé et si to est une famille faiblement lisse), et si to vérifie la propriété :

(CF) $\quad \operatorname{CF}\left(t_{t}\right)<\omega_{1}$ et $\operatorname{co}\left(t_{t}\right)<\omega_{2}$.

Alors toute famille de parties d'un $\varphi$-espace $E$, de type $A_{0}$, contient une famille $\varphi$-dis jointe de type $t 6$.

Ce théorème résulte de 5.3 et du :

Corollaire.- Toute famille $\left(x_{i}\right)$ de parties de rang infini d'un $\varphi$-espace $E$ telle que $\left(r\left(x_{i}\right)\right)$ soit lisse (resp. telle que $\left(r\left(x_{i}\right)\right)$ soit faiblement lisse et si on admet $l$ 'hypothèse du continu généralisé) et telle que en outre $\left(\mathbf{r}\left(\mathbf{x}_{\mathbf{i}}\right)\right)$ vérifie la propriété $(C F)$, contient une famille disjointe $\left(\mathrm{Y}_{\mathbf{i}}\right)$ telle que : $\forall i \in I, \quad r\left(x_{i}\right)=r\left(Y_{i}\right)$

Toute famille dénombrable de cardinaux infinis est faiblement lisse et vérifie la propriété (CF) . En outre, on verra au lemme 4 que l'hypothèse du continu généralisé $n$ 'est plus nécessaire quand pour le cardinal, dénombrable, et pour 'les $a_{i}$ non dénombrables, la famille est lisse. D'où le résultat indépendant de l'hypothèse du continu généralisé : 
Théorème 3.- Toute famille dénombrable $\left(x_{i}\right)$ telle que $r\left(x_{i}\right)$ soit infini pour tout $i$ contient une famille disjointe $\left(Y_{i}\right)$ telle que :

$$
\forall i \in I \quad r\left(Y_{i}\right)=r\left(x_{i}\right)
$$

Pour démontrer le corollaire et le théorème 3 , l'on va d'abord établir quelques lemmes de théorie des ordinaux et des cardinaux, puis l'on utilisera une récurrence transfinie sur $L$ pour construire $\left(Y_{i}\right)$.

\subsection{2.- Applications et familles fortes}

Soit $t_{b}=\left(a_{i}\right)_{i \in I}$ une famille de cardinaux infinis.

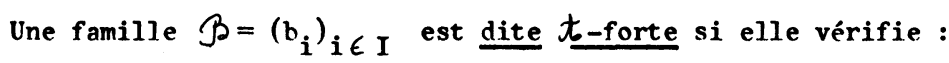

$\left(\mathrm{FC}_{1}\right) \quad \forall i \in I, \quad U_{0} \leq b_{i}<a_{i}$ ou $a_{i}=U_{0}=b_{i}$

et

$$
\begin{aligned}
& \left(\mathrm{FC}_{2}\right) \quad \forall \ell \in \mathrm{L}_{\mathbb{t}}, \quad \forall \mathrm{J}<\mathrm{UI} \mathrm{I}_{\mathrm{m}}[\mathrm{m}<\ell] \text { tel que } \operatorname{Sup}_{\mathrm{i}}[\mathrm{i} \in \mathrm{J}]=\ell \\
& \text { on } a \quad \quad \quad \quad \sup b_{i}[i \in J]=\ell
\end{aligned}
$$

et

$\left(\mathrm{FC}_{3}\right)$ Si $\mathrm{L}_{f_{0}} \mathrm{n}^{\prime} \mathrm{a}$ pas de plus grand élément, $\forall J \subset I$ tel que $\left\{a_{i} \mid i \in J\right\}$ soit cofinal à $L_{t}, \quad\left\{b_{i} \mid i \in J\right\}$ est cofinal à $L_{t}$

La famille $A_{6}$ est dite forte s'il existe au moins une famille $\mathscr{B}$ qui est $t_{b}$-forte.

Un ordinal $\alpha$ est dit fort si la famille $\left(U_{\nu}\right)_{\nu \in \alpha}$ est forte, i. e. s'il existe une application $f$ de $\alpha$ dans lui-même ayant les propriétés :

$$
\left(\mathrm{FO}_{1}\right) \quad f(0)=0 \text { et pour tout } \xi \in \alpha-\{0\}, f(\xi)<\xi
$$

et

$\left(\mathrm{FO}_{2}\right) \quad \forall \xi \in \alpha+1, \forall 0<\xi$ cofinal ̀े $\xi, \mathrm{f}(0)$ est cofinal ̀̀ $\xi$. Une telle application $f$ est dite $\underline{\alpha-f o r t e}$. Cette définition se transporte à un ensemble $E$ hien ordonné, d'où $E$ fort et $f$ F-forte. 
Lemme 1.- Un ordinal $\alpha$ est fort si et seulement si $\alpha<\omega_{1}$, i.e. si et seulement si $\alpha$ est fini ou dénombrable

démonstration

a) $\omega_{1}$ n'est pas un ordinal fort.

En effet s'il existe une application $f \omega_{1}$-forte de $\omega_{1}$ dans luimême, soit $\alpha_{1}=1$,

$$
\begin{array}{ll}
\alpha_{2}=\operatorname{Min} \xi & {\left[\forall \eta \in \omega_{1}-\xi, f(\eta)>\alpha_{1}\right]} \\
\ldots & \\
\alpha_{n}=\operatorname{Min} \xi & {\left[\forall \eta \in \omega_{1}-\xi, f(\eta)>\alpha_{n-1}\right]} \\
\ldots &
\end{array}
$$

$$
\text { Daprès }\left(\begin{array}{ll}
F & 0_{1}
\end{array}\right) \text { et }\left(\begin{array}{ll}
F & 0_{2}
\end{array}\right) ; \quad \alpha_{n} \text { existe pour tout } n \in \omega \text { et : }
$$

$\alpha_{1}<f\left(\alpha_{2}\right)<\alpha_{2}<f\left(\alpha_{3}\right)<\ldots$

donc $\quad\left(\alpha_{n}\right)_{n \in \omega}$ est strictement croissante, et :

$$
\forall \alpha \in \omega_{1}, \operatorname{si} f(\alpha) \leqslant \alpha_{n} \quad, \quad \alpha<\alpha_{n+1}
$$

On en déduit par récurrence transfinie sur $\alpha$ que tout $\alpha \in \omega_{1}$ est inférieur à un $\alpha_{n}$ au moins. $\left(\alpha_{n}\right)_{n \in \omega}$ serait donc cofinal à $\omega_{1}$, d'où la contradictión (ces raisonnements sont identiquẹs à ceux de $[0,1,3], \S 6$, exercice 23 ).

b) Si $\left(\alpha_{\nabla}\right)_{\nu e \alpha}$ est une famille d'ordinaux forts $>0$, indiciée par un ordinal fort, alors $\sum_{v \in \alpha} \alpha_{v}$ est un ordinal fort.

(*) $\omega_{1}-\xi$ est une difference ensembliste ici 


$$
\text { En effet soit } F=\sum_{\nu \in \alpha} \alpha_{\nu}=\beta_{\alpha} \text { et } \forall \nu \in \alpha, \beta_{\nu}=\sum_{\lambda \in \nu} \alpha_{\lambda}
$$

Alors, $\forall \nabla \in \alpha \quad$ Ord $\left(\left[E_{\nu}, E_{v+1}[)=\alpha_{v}\right.\right.$

et $\quad\left(\left[E_{v}, E_{v+1}[)_{v \in \alpha}\right.\right.$ est une partition de $\beta$

Donc : $\forall \lambda \in \beta, \exists v \in \alpha, v$ unique tel que $\lambda \in\left[\beta_{v}, \beta_{\nu+1}[\right.$

on écrit $v(\lambda)$ cet ordinal.

Soit $f^{\alpha}$ une application $\alpha$-forte et pour tout $v \in \alpha$, soit $f_{v}$ une application $F_{\nabla}, E_{v+1}[$-forte. Alors l'application $f$ de $\beta$ dans lui-même définie par :

$$
\begin{aligned}
\forall \lambda \in \beta \quad & \text { ou } \lambda>E_{v(\lambda)} \text { et } & f(\lambda)=f_{v(\lambda)}(\lambda) \\
& \text { ou } \lambda=R_{v(\lambda)} \neq 0 \text { et } & f(\lambda)=R_{f^{\alpha}(v(\lambda))}
\end{aligned}
$$

est une application f-forte.

$$
\begin{aligned}
& \left(F 0_{1}\right) \text { est trivialement vérifié. Démontrons }\left(F 0_{2}\right) \text { : } \\
& \forall \lambda \in F+1, \quad \forall 0 \subset \lambda, 0 \text { cofinal à } \lambda
\end{aligned}
$$

ou bien $\lambda>\beta_{v(\lambda)}$ et $\quad 0^{\prime}=0 . \cap(] \beta_{v(\lambda)}, \lambda[)$ est cofinal a $\lambda$, donc $f\left(0^{\prime}\right)=f_{v(\lambda)}\left(0^{\prime}\right)$ est cofinal à $\lambda$, et à fortiori $f(0)$

ou bien $\quad \lambda=\beta_{v(\lambda)}$ où $\quad v(\lambda) \in \alpha+1$ et $v(\lambda)$ a un prédecesseur. Alors soit $\left.U_{\lambda}=\right] \beta_{v(\lambda)-1}, \beta_{v(\lambda)}\left[\quad 0 "=0_{n} U_{\lambda}\right.$ est

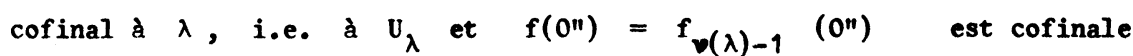
à $u_{\lambda}$, i.e. à $\lambda$, donc a fortiori $f(0)$ 
ou bien $\lambda=\beta_{v(\lambda)}$ où $\quad v(\lambda) \in \alpha+1$ et $v(\lambda) \quad$ n'a pas dé prédecesseur.

$$
\begin{aligned}
\text { Alors soit } & 0_{1}=\left\{v / v \in v(\lambda) \text { et } \beta_{\nabla} \in 0\right\} \\
\text { et } & \left.0_{2}=\left\{v / v \in v(\lambda) \text { et } 0 \cap(] \beta_{\nabla}, \beta_{\nu+1}\right) \neq \emptyset\right\} .
\end{aligned}
$$

$0_{1}$ ou $0_{2}$ est cofinal à $v(\lambda)$

Si $0_{1}$ est cofinal à $v(\lambda)$ :

Sup $f(0) \geqslant \operatorname{Sup} f\left(\beta_{v}\right)\left[v \in 0_{1}\right] \geqslant \operatorname{Sup} \beta_{f^{\alpha}(\nu)}\left[v \in 0_{1}\right] \geqslant \beta_{v(\lambda)}$

Si $0_{2}$ est cofinal à $v(\lambda)$ :

$\forall v \in 0_{2} \quad, \quad \exists \gamma_{v} \in 0_{:} \cap \cap(] \beta_{v}, \beta_{v+1}[) \quad$ et

Sup $f(0) \geqslant \operatorname{Sup} f\left(\gamma_{v}\right)\left[v \in 0_{2}\right] \geqslant \operatorname{Sup} f\left(\beta_{v}\right)\left[v \in 0_{2}\right] \geqslant \beta_{v(\lambda)}$

et comme $f(0) \subset \beta_{V(\lambda)}=\lambda \quad, f(0)$ est cofinal à $\lambda$.

c) Tout ordinal dénombrable est fort

Tout ordinal fini est trivialement fort. $\omega$ est fort car

$$
\left\{\begin{array}{l}
f(n)=n-1 \quad \text { si } n>0 \\
f(0)=0
\end{array}\right.
$$

est $w$-forte.

Il est alors aisé de démontrer par récurrence transfinie dénombrable qque tout ordinal dénombrahle $\alpha$ est fort.

Supposons en effet que tout $f<\alpha$ soit fort et $\alpha$ dénombrable.

Si $\alpha$ a un prédécesseur $\alpha-1, \alpha=(\alpha-1)+1$

Si a n'a pas de prédécesseur, le caractère final de $\alpha$ est $\omega$, et l'on peut construire une famille cofinale à $\alpha$ ainsi :

soit $n \longrightarrow \xi_{n}$ une bijection de $\omega$ sur $\alpha$ et soit : 


$$
\begin{aligned}
& \eta_{1}=\xi_{1} \\
& \cdots \ldots \ldots \ldots \\
& \eta_{n}=\operatorname{Min}\left(\xi_{k}\right)\left[\xi_{k}>\eta_{n-1}\right]=\xi_{f(n)}
\end{aligned}
$$

$\left(\eta_{n}\right)_{n \in \omega}$ est strictement croissante et cofinale à $\alpha$

(voir fo), Livre I, chap. II, 39, page 83). D'où :

$$
\alpha=\sum_{n \in \omega} \text { ord }\left(\eta_{n+1}-\eta_{n}\right) \quad \text { en posant } \eta_{0}=0
$$

Donc $\alpha$ est toujours somme d'ordinaux forts $>0$, indiciée par un ordinal fort, et d'après b) $\alpha$ est fort.

d) Les ordinaux $\alpha \geqslant \omega_{1}$ ne sont pas forts

En effet tout ordinal inférieur à un ordinal fort est lui-même fort, d'où le résultat en utilisant a).

e) Construction d'applications fortes.

exemple d'application $\omega^{\omega}$-forte :

$$
f(p \omega+q)=\left\{\begin{array}{l}
(p-1) \omega \quad \text { si } q=0 \\
p \omega+q-1 \text { si } q \geq 1
\end{array} \quad(p \in \omega \text { et } q \in \omega)\right.
$$

$$
\begin{aligned}
& f\left(\omega^{2}\right)=\omega, f\left(\omega^{2}+\alpha\right)=\omega^{2}+f(\alpha) \text { si } 0<x<\omega^{2} \\
& \ddot{f}\left(\omega^{n}\right)=\omega^{n-1}, f\left(\omega^{n}+\alpha\right)=\omega^{n}+f(\alpha) \quad \text { si } \quad 0<\alpha<\omega^{n} \\
& \ddot{f}\left(\omega^{\omega}\right)=\omega
\end{aligned}
$$


FAMILLES DISJOINTES INCLUSES (INFINIES)

135

D'une manière générale soit $\alpha$ un ordinal et

$$
\alpha_{\omega}=\left\{\xi / \xi \quad \text { ordinal et } \omega^{\xi}<\alpha\right\}
$$

Si $g$ est une application $\alpha_{\omega}$-forte, on construit une application $f$-forte ainsi :

$$
\forall \lambda \in \alpha \text {, soit } \lambda=n_{1} \omega^{\xi_{1}}+\ldots+n_{p} \omega^{\xi_{p}}
$$

la décomposition polynomiale de $\lambda$ (voir $[\mathrm{C}, \mathrm{I}, 3], \S 6$, exercice 12, page 100) où $n_{i}$ sont des entiers $>0$, p est un entier $>0$ et $\xi_{1}, \ldots, \xi_{p}$ sont des ordinaux $\geq 0$ strictement décroissants. Alors :

$$
f(\lambda)=n_{1} \omega^{\xi_{1}}+\ldots+n_{p-1} \omega^{\xi_{p-1}}+\left(n_{p}-1\right) \omega^{\xi}+\omega^{g\left(\xi_{p}\right)}
$$

C Q F D

lemme 2.- Soit $A$ une famille de cardinaux infinis.

si $\quad C F(t)>\omega_{1}, A_{t}$ 'est pas corte

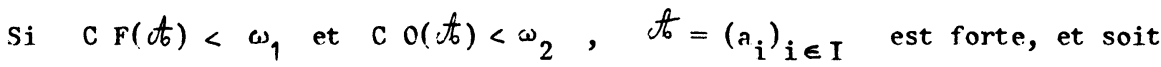

$$
\begin{aligned}
& \mathrm{L}=\left\{\mathrm{a}_{\mathrm{i}} / \mathrm{i} \in \mathrm{I}\right\} \quad \text { et } \mathscr{L}=(\ell)_{\ell \in \mathrm{L}} \text {. Nlors il existe } \\
& \mathscr{C}=\left(\mathrm{c}_{\ell}\right)_{\ell \in \mathrm{L}} \quad \mathscr{L} \text {-forte telle que pour tout } \ell \in \mathrm{L}, \\
& \text { Card }\left(\left\{\mathrm{m} / \mathrm{m} \in \mathrm{L}, \mathrm{m}>\ell \quad \text { et } \mathrm{c}_{\mathrm{m}}<\ell\right\}\right)<\operatorname{Min}\left(U_{2}, \ell\right)
\end{aligned}
$$

Démonstration

a) $t=\left(a_{i}\right)$ est forte si et seulement si $\mathscr{L}=(l)_{\ell \in I}$ est forte

$$
\text { S'il existe } \beta=\left(b_{i}\right) \quad \text { Af-forte, sojt pour } l \in L \quad i(l) \in I_{l} \text {. }
$$

Alors $\ell=\left(h_{i}(\ell)\right)_{\ell \in L} \quad$ est $\mathscr{L}$-forte.

Inversement si $\mathscr{L}$ est forte, il existe $\mathscr{C}=\left(\mathrm{c}_{\ell}\right) \mathcal{L}$-forte ; donc si l'on pose pour tout $i \in I, \ell(j)$ tel oue $i \in I_{\ell}(i)$, 


$$
B=(C \ell(i))_{i \in I \quad \text { est }} \text { of-forte. }
$$

b) Si L est forte, CF $(t) \leqslant \omega_{1}$

Fn effet si $\mathcal{L}$ est forte, il existe $\beta=\left(b_{\ell}\right) l \in L \quad \mathcal{L}$-forte et si $C F(t)>\omega_{1}$, il existe $M \in I$ tel que $M U\{$ Sup $M$ p soit fermé et Ord $M=\omega_{1} \quad$ (donc $M$ non fermé).

Soit $\longrightarrow m_{v} \quad l$ 'isomorphisme canonique de $\omega_{1}$ sur $M$

$$
f: v \longrightarrow f(v)=\sup \lambda \quad\left[\lambda e \omega_{1} \text { et } m_{\lambda} \leqslant b_{m_{v}}\right]
$$

où l'on convient Sup $\lambda[\lambda \in \varnothing]=0$, est une application $\omega_{1}$-forte

$$
\begin{aligned}
& \text { En effet } f(0)=0 \text { et pour tout } v \in \omega_{1}-\{0\}, \\
& m_{f(v)} \leqslant b_{m_{v}}<m_{v}, \quad \text { donc } f(v)<v
\end{aligned}
$$

En outre :

$$
\forall \xi \in \omega_{1}+1, \forall 0 \in \xi \quad, 0 \text { cofinal à } \xi \text {, }
$$

On a Sup $m_{\lambda} \quad[\lambda \in 0]=m_{\xi} \quad\left(m_{\omega_{1}}=\operatorname{Sup} M\right)$

donc Sup $b_{m_{\lambda}} \quad[\lambda \in 0] \quad=m_{\xi}$

et Sup $f(0)=\sup \lambda\left[\lambda \in \omega_{1}\right.$ et $\left.\exists v \in 0, m_{\lambda} \leqslant b_{m_{v}}\right]=\xi$

D'où la contradiction car $\omega_{1}$ n'est pas un ordinal fort.

c) $\mathrm{Si} \mathrm{CF}(\mathcal{L})<\omega_{1}$ et $\mathrm{CO}(\mathcal{L})<\omega_{2}, \mathcal{L}$ est forte.

Soient $S(L)$ l'ensemble des a $\in L$ tels que : 
a $=\operatorname{Min} \mathrm{L}$ ou Sup $\ell \quad[l \in \mathbf{L}$ et $\ell<\mathrm{a}]<\mathrm{a} \quad$ (1),

$R(L)$ l'ensemble des $a \in I$, a sans prédecesseur, $\bar{a}>U_{0}\left(^{*}\right)$ et tel que :

$$
\text { Sup } \ell \quad\left[\ell \in \mathbf{L}-S_{(}(\mathrm{L}) \text { et } \ell<\mathrm{a}\right]<\mathrm{a}
$$

et $\quad I(I)=S(L) \cup R(L)$

L'on peut alors définir $\quad L_{0}=I(L), L_{1}=I\left(L-L_{0}\right), \ldots$,

$$
\mathrm{I}_{v}=I\left(\mathrm{~L}-\bigcup_{\lambda \in v} \mathrm{~L}_{\lambda}\right), \ldots
$$

et l'on note $\gamma$ le plus petit ordinal tel que $L_{\gamma}=\emptyset$

$$
\left(L_{v}\right)_{v \in X} \text { est alors une partition de } L \text {. }
$$

Soit $b=$ Sup $L$ Dans l'ensemble $E_{b}$ des cardinaux infinis $\leqslant b$, un cardinal $a \in \mathrm{L}$ est de trois types possibles :

type I : a $=\hat{U}_{0}$ ou a a un prédécesseur. Alors $a \in S(\mathrm{~L})$ et a $\in \mathrm{I}_{0^{\circ}}$

tyoe II : a n'a pas de prédécesseur et $\bar{a}=u_{0}$.

Si a n'a pas de prédécesseur dans L, ou plus généralement si l'on a $(1), a \in L_{0} ;$ sinon $a \in L_{v}$ pour $v>0$.

Soit $M$ une partie fermée de $L$ telle que ord $M=\omega^{v}+1$, et $\lambda \longrightarrow m_{\lambda}$ I'isomorphisme canonique de $\omega^{v}+1$ sur $M$. Pour tout $\lambda \in \omega^{\nu}+1, \lambda$ sans prédécesseur, $m_{\lambda}$ est de type II. En outre on a Sup $M=\operatorname{Max} M \in L^{\nu}, \quad C F(\mathcal{L})>\omega^{v}$, d'où, posant $\gamma-=\operatorname{Sup} \lambda[\lambda \in \gamma]$, on a :

$$
\omega^{\gamma-} \leq \text { c F }(\mathcal{Z}) \leq \omega^{(\gamma-)+1}
$$

et en particulier $\quad x<\omega_{1}$

Soit $a \in I$, de type $I$ ou de type II. Il existe $v \in \gamma$ tel que $a \in L_{v}$ et, dans $L^{v}=L-\bigcup_{\lambda \in v} L_{\lambda}, \quad$ on $a$ : 
$a-=\sup l\left[l \in L_{v}\right.$ et $\left.l<a\right] \leqslant \sup l \quad\left[l \in L^{\nu}\right.$ et $\left.l<a\right]<a$ Donc a $-<a$ et il n'existe pas de cardinal $l \in L_{v}$ tel que : a $<\ell<a$. En particulier a- est le prédécesseur de a dans $L_{v}$, s'il existe

type III a n'a pas de prédécesseur et $\bar{a}>U_{0}$

type III a : a n'est pas limite dans $L$, i.e. a a un prédécesseur asinon $a \in L_{v}$ et il résulte de (2) dans $L^{\nu}$ que, soit :

$$
\text { a - }=\sup l \quad\left[\ell \in \mathrm{L}^{\nabla}-S\left(\mathrm{~L}^{\nu}\right) \text { et } \ell<\mathrm{a}\right]
$$

on a : a $a<a$

et

$$
N=] a-, a\left[\cap L^{\nu}=\right] a-, a\left[\cap L_{v}\right.
$$

vérifie $C F(N) \leqslant \omega_{0}$, ou mieux $\bar{N} \cap L=N$. L'on exprime encore ceci en disant que $\mathrm{N}$ est discret pour la topologie $\mathcal{C}_{0}$ définie sur $\mathrm{I}$ par la fermeture :

$$
M \longrightarrow \bar{M}=\left\{m / m \in L \quad \text { et } \exists N \subset M, \text { Card } N \leq i l_{0} \text { et Sup } N=m\right\}
$$

Alors on définit :

$$
\mathrm{a}^{+}=\sup \ell\left[\ell \in \mathrm{R}\left(\mathrm{I}^{\nu}\right) \text { et }[\mathrm{a}-, \ell] \cap \mathrm{L}^{\nu} \text { est } \ell_{0} \text { discrète }\right]
$$

On a

$$
a-<a \leq a+
$$

Il résulte de (1) dans $L^{\nu}$ que $\mathrm{N}$ est cofinal à a .

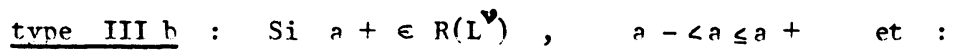

$$
\left.\left.\left.\mathrm{v}_{\mathrm{a}}=\right]_{a-}, \mathrm{a}^{+}\right] \cap \mathrm{L}^{\nu}=\mathrm{Ja-}, \mathrm{a}^{+}\right] \cap \mathrm{L}_{\boldsymbol{v}} \text { est } \mathscr{C}_{\mathrm{o}} \text {-discret. }
$$


type III c : Si $a+\notin R\left(L^{\nu}\right), \quad a-<a<a+$ et :

$$
\left.N_{a}=\quad\right] a-, a+\left[\cap L^{\nu}=\right] a-, a+\left[\cap L_{v} \text { est } e_{0}\right. \text {-discret, }
$$

Dans les deuxples pour tout $\lambda \in \gamma, \lambda>v$, on a $L_{\lambda} \cap N_{a}=\varnothing$.

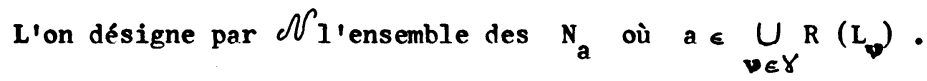

c1) Toute famille $\mathscr{L}_{0}=(\ell)_{\ell \in N}$ où $N$ est $\ell_{0}$-discret et Card $N<\omega_{2}$ est forte.

Ceci se démontre aisément par récurrence transfinie sur $\alpha=$ ord $\mathrm{N}$, en considérant une famille $\left(\alpha_{\vartheta}\right)_{\vee \in \omega_{\beta}}$ strictement.croissante et oofinale à $\alpha$ d'ordinaux de $\alpha$ (c'est trivial si $\alpha$ a un prédécesseur), et en utilisant un procédé analogue à celui de la démonstration du lemme $1, b)$, sachant que, $\longrightarrow \ell_{\boldsymbol{v}}$ étant l'isomorphisme de $\alpha \operatorname{sur} N,\left(\ell_{\alpha_{v}}\right) v \in \omega_{\beta}$ est forte. En effet, c'est trivial si $\omega_{\beta}=\omega_{0}$. Si $\omega_{\beta}=\omega_{1}$, comme on peut toujours choisir les $\alpha_{v}$ de caractère final dénombrable au plus, il suffit de prendre :

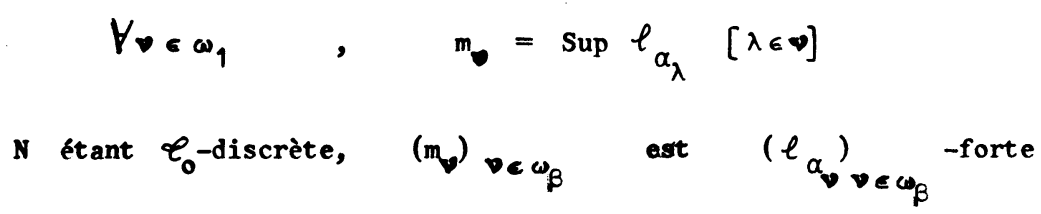

Remarque : Un raisonnement identique à celui du lemme 1, démonstration, a) permet de démontrer qu'une famille $\mathscr{L}_{0}=(l)_{\ell \in N}$ où $\mathrm{N}$ est $\ell_{0}$-discret, mais $\ell_{1}$-fermé, où $\ell_{1}$ est la topologie définie par la fermeture :

$$
X \longrightarrow \bar{X}=\left\{x / x \leq S \text { Sup } X \text { et } \exists Y \subset X, \text { Sup } Y=x \text { et } \operatorname{cf}(X)=\omega_{1}\right\} \quad \text { U X }
$$

ou cf $(X)$ est le caractère final de $X$.

(Remplacer $\omega_{1}$ par $\omega_{2}$ et $\omega_{0}$ par $\omega_{1}$ dans les raisonnements, la suite $\left(\alpha_{n}\right)_{n \in \omega_{1}}$ serait alors cofinale à $\left.\omega_{2}\right)$. 


\section{$c_{2}$ Construction d'une famille $\mathscr{L}$-forte}

Soit $f$ une application $\gamma$-forte et pour tout $\mathrm{N} \in \mathcal{N}$,

une famille $\left(c_{\ell}(\mathrm{N})\right) \quad l \in N \quad(l)_{l \in N^{- \text {forte }}}$

$$
\text { Soit alors } \quad l \in \mathbf{L}: l \in \mathrm{I}_{\lambda}
$$

Si $l$ est de type $I, \quad c_{l}=\ell-$

Si $\ell$ est de type II, soit $\left.\left.N_{\ell}=\right] \ell-, \ell\right] \cap \mathrm{L}$. L'on peut faire pour $N$ la partition $\left(\mathrm{N}_{\ell, \nu}\right)_{\nu \in \lambda+1}$, comme pour $\mathrm{L}$; l'on a $\operatorname{Min} \mathrm{N}_{\ell, \lambda}=\ell$ et l'on pose :

$$
c_{\ell}=\operatorname{Min}{ }^{N}, f(\lambda)
$$

Si $l$ est de type III a, l'on pose $c_{\ell}=\ell_{-}$

Si $\ell$ est de type III b ou III c, l'on pose $c_{\ell}=c_{\ell}(\mathrm{N})$

où $N \in \mathcal{N}$ est tel que $l \in N$, quạnd $l$ n'est pas borne supérieure dans $\mathrm{N}_{\mathrm{a}} \cdot$

$\left(F c_{1}\right)$ est trivialement vérifié

Vérifions $\left.\left(F c_{2}\right): \forall l \in L, \forall 0 c\right] \leftarrow, l[n \mathrm{~L}$ tel que sup $0=\ell$, a-t-on $\operatorname{Sup}_{\text {.c }}[\ell \in 0]=l$ ? $\quad l$ ne peut être que de type II ou III.

$$
\left.l \in I_{\nu} \text { et } 0^{\prime}=0 \cap\right] \ell-, l\left[\text { est cofinal à } l: 0^{\prime} c \underset{\lambda \in \nu}{\cup} L_{\lambda}\right.
$$

\section{Si $\ell$ est de type II :}

ou bien il existe $\lambda \in D$ tel que $O \cap I_{\lambda}$ soit cofinal à $l$ et deux cas :

$\left.1^{\circ}\right)$ il existe une famille strictement croissante $\left(\ell_{n}\right)_{n \in \omega}$ de cardinaux de type I, II ou III a dans $0 \cap \mathrm{L}_{\lambda}$ telle que sup $\ell_{n}=\ell$. Alors $\ell_{n} \leq c^{c} \ell_{n+1}$ pour tout $n$, et $\sup c_{\ell_{n}}=\ell$

20) il existe une famille strictement croissante $\left(l_{n}\right)_{n \in \omega}$ de cardinaux de type III b ou III c telle que Sup $\ell_{n}=l$. 
A partir d'un certain rang tous les $\ell_{n}$ sont de type III c et situés dans un NeN, $\ell=\ell_{n}+$, donc $\operatorname{Sup} c_{l_{n}}(\mathrm{~N})=\ell$

ou bien pour tout $\lambda \in \mathcal{O}, O \cap \mathrm{L}_{\lambda}$ n'est pas cofinal à $l$.

Alors il existe une suite strictement croissante $\left(\nu_{n}\right)_{n \in \omega}$ telle que sup $\nu_{n}=0$, et une suite strictement croissante $\left(\ell_{n}\right)_{n \in \omega}$ telle que $\ell_{n} \in L_{\nu_{n}}$ pour tout $n$ et Sup $\ell_{\mathrm{n}}=\ell$.

Comme $\ell_{n}<\ell_{n+1}$ et $\nu_{n}<\nu_{n+1}$, on a $\ell_{n+1} \leq l_{\bar{n}}$, et comme la suite $\left(\ell \bar{n}_{n \in \omega}\right.$ ne peut décroitre indéfiniment, à partir d'un rang $p$, on a $l_{\bar{n}}=l_{\bar{p}}=l_{-}$pour tout $n \geqslant p$, donc pour tout $n \in \omega$,

$$
\ell_{n}=\operatorname{Min}\left(\mathrm{N}_{\ell, \nu}\right)
$$

et $\mathrm{f}$ étant $\gamma$-forte, on $\mathrm{a}$ :

$$
\text { Sup } \ell_{n}=l \Rightarrow \sup c_{l_{n}}=l
$$

Si $l$ est de type III :

$$
\text { Alors } l \in L_{\nu} \text { et, comme Card } \nu \leqslant U_{0} \text {, il existe } \lambda \in \nu \text { tel que }
$$

Sup $O \cap I_{\lambda}=l$, et en outre $\left.0^{\prime}=0 \cap I_{\lambda} \cap\right] l-, l[$

Sup $0^{\prime}=\ell$

Or, pour tout $n \in 0^{\prime}, \quad m_{n}=\operatorname{Min}\left\{m / m \in N_{n}\right.$ et $\left.m>n\right\} \quad$ est tel que

$$
c_{n}\left(N_{l}\right)<n<m_{n} \quad \text { et } \quad c_{n}\left(N_{\rho}\right)<c_{n}<m_{n}
$$

donc $l=\operatorname{Sup} m_{n}\left[n \in 0^{\prime}\right]=\operatorname{Sup} n\left[n \in 0^{\prime}\right]=\operatorname{Sup}_{n}\left(N_{l}\right)\left[n \in 0^{\prime}\right]=\operatorname{Sup} c_{n}\left[n \in 0^{\prime}\right]$

C Q F D

$c_{3\}}$ Pour tout $l \in \mathrm{L}, \mathrm{c}_{\ell}=\left\{\mathrm{m} / \mathrm{m} \in \mathrm{L}, \mathrm{m}>\ell\right.$ et $\left.\mathrm{c}_{\mathrm{m}}<\ell\right\}$ a pour cardinal $U_{1}$ au nlus

En effet soit $l \in L_{\nu}$. Si $\lambda \in \nu, \quad c_{\ell}{ }^{n L_{\lambda}}=\varnothing$. 
Si $\lambda \geqslant \nu$ :

il existe au plus un $m>\ell$ de type I, II ou III a dans $L_{\lambda}$ tel que $m-<\ell$, donc tel que $f(\ell)<\ell$ éventuellement.

il existe au plus un $\mathrm{N} \in \mathcal{N}, \mathrm{N} \subset \mathrm{L}_{\lambda}$ tel que $\left.\mathrm{N}=]_{a}, \mathrm{~b}\left[\cap \mathrm{L}_{\lambda} \text { ou } \mathrm{N}=\right]_{\mathrm{a}, \mathrm{b}}\right] \cap \mathrm{L}_{\lambda}$ et $\mathrm{a} \leqslant l \leqslant \mathrm{~b}$.

Donc Card $\left(c_{l} \cap L_{\lambda}\right) \leqslant U_{1}$

D'où card $c_{l} \leqslant U_{1}$ pour tout $l$

En outre on peut toujours choisir $c_{l} \geq U_{1}$ pour tout $l>U_{1}$, d'où le résultat du lemme 2 .

Conjecture : Il est fortement probable aue le leme 2 puisse être etendu au cas où $\mathrm{C} F(t)=\omega_{1}$. 


\subsection{3. - Autres lemmes préliminaires}

Soit $E$ un ensemble de cardinal infini a et $\mathscr{E} l$ 'ensemble des parties $\mathcal{H}$ de $\beta$ (E) vérifiant :

(S E P). $\forall \mathbf{X} \in \mathcal{H}, \forall \mathbf{Y} \in \mathcal{H}, \mathbf{X} \neq \mathbf{Y}, \operatorname{Card} \mathbf{X}=\operatorname{Card} \mathbf{Y}=\mathbf{a}$ et $\operatorname{Card}(\mathbf{X} \cap \mathbf{Y})<\mathbf{a}$.

Le cardinal :

$$
\mathbf{a}^{\mathbf{s}}=\operatorname{Sup}(\text { Card } \mathscr{H}) \quad\left[H \in\left[\begin{array}{lll}
H & \in
\end{array}\right]\right.
$$

est appellé le degré de séparabilité de a.

Il est aisé de vérifier que cette borne supérieure est atteinte quand $a^{s} n^{\prime}$ est pas un cardinal inaccessible, et le fait que $a^{r}=a$ entraine $a^{s} \geq a$.

lemme 3.- Pour tout cardinal infini a, $a^{s} \geq a$. En outre, pour tout cardinal

a tel que :

$$
\text { Sup } 2^{b}[b<a] \leq a \quad \text {, on } a \quad a^{s}=2^{a}>a \text {. }
$$

C'est en particulier toujours le cas si l'on admet l'hypothèse du continu généralisé, et alors la borne supérieure est toujours atteinte.

Démonstration : Soit $a=V_{\alpha}$ un cardinal infini tel que

$$
\begin{gathered}
\text { Sup } 2^{b}[b<a] \leq a \\
\text { Alors } E=U 2^{\lambda}\left[\lambda \in \omega_{\alpha}\right] \quad \text { est un ensemble de cardinal : } \\
\text { Card } E=\sum_{\lambda \in \omega_{\alpha}} 2^{\operatorname{card}(\lambda)}=\sum_{b<a} 2^{b}=a
\end{gathered}
$$

Considérons alors pour toute $f \in 2^{\omega^{\omega}} \alpha$, l'ensemble $H_{f}$ des restrictions de $f$ aux $\lambda \in \omega_{\alpha^{*}}$ Alors $\mathcal{H}=\left\{\mathrm{H}_{\mathrm{f}} / \mathrm{f} \in 2^{\omega} \alpha\right\}$ vérifie la propriété (S E P) pour E, donc

$$
a^{s} \geqslant 2^{a}
$$

Comme on a trivialement $a^{s} \leqslant 2^{a}: \quad a^{s}=2^{a}$ 
Si on admet 1 'hypothèse du continu généralisé, pour tout cardinal infini a, Sup $2^{b}[b<a]=a$, donc $a^{s}=2^{a}=a+$ successeur de $a$. lemme $40-$ Soit $\left(x_{i}\right)_{i \in I}$ une famille de parties d'un $\varphi$-espace $E$ telle que, a étant un cardinal infini on a :

$$
\forall i \in I \quad, \quad r\left(x_{i}\right) \geqslant a \quad \text { et } \quad \text { Card } I<a^{s}
$$

Alors il existe une famille $\left(Y_{i}\right)_{i \in I}$ disjointe, incluse dans $\left(X_{i}\right)$ telle que $r\left(Y_{1}\right)=a$ pour tout $i$.

Cette propriété est encore vraie si Card $I=a$ quand a $n$ 'a pas de prédécesseur

\section{Démonstration}

a) Si a est régulier et Card $I<a^{s}$, toute famille $\left(x_{i}\right)_{i \in I}$ telle que $r\left(x_{i}\right) \geq a$ pour tout $i$, contient une famille $\varphi$-disjointe de type a.

$$
\text { Soit } a=U_{\alpha} \text { et } \operatorname{Card} I=U_{\beta}<a^{s} \text {. L'on considère } I=\omega_{\beta}
$$

$(\beta \leq \alpha)$. D'où $\left(x_{\lambda}\right)_{\lambda \in \omega_{\beta}}$

L'on va construire par récurrence transfinie sur $\lambda \in \omega_{\beta}$ une partie $T_{\lambda}$ de cardinal a de $x_{\lambda}$, de sorte que :

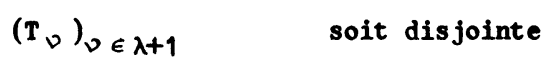

et

$$
\forall \nu \in \omega_{\beta} \quad, \quad \nu>\lambda \quad r\left[\bigcup_{\mu \leq \lambda} T_{\mu}\right] \quad\left(x_{\nu}\right) \geqslant a
$$

Soit $\lambda \in \omega_{\beta}$ et supposons que l'on aic défini $T_{\mu}$ pour $\mu \in \lambda$ ayant les propriétés ci-dessus. En vertu du caractère fini de la disjonction,. $\left(T_{\nu}\right)_{\nu \in \lambda}$ est disjointe. En outre, comme a est régulier et Card $\lambda<$ Card $I \leqslant a$, en vertu du lemme 5 ci-dessus, 


$$
\begin{aligned}
\text { si } \quad r^{\prime}=r\left[\bigcup_{\mu \in \lambda} T_{\mu}\right], \\
\forall \nu<\omega_{\beta}, \quad \nu \geqslant \lambda \quad, \quad r^{\prime}\left(x_{\nu}\right) \geq a
\end{aligned}
$$

En particulier il existe une partie $r^{\prime}$-libre $z$ de cardinal a de $x_{\lambda}$. Soit alors $\mathcal{H}$ une partie de $\bar{q}(z)$, vérifiant (S E P) et telle que Card $H>$ Card I . Or pour tout $\nu \in \omega_{\beta}, \nu>\lambda$, il existe au plus un $\mathrm{T} \in \mathcal{H}$ tel que

$$
r_{T}^{\prime}\left(x_{0}\right)<a
$$

(il n'en existe certainement pas si $r\left(X_{v}\right)>a$ ), en vertu du lemme $1 d u$ chapitre IV, et l'on a :

$$
\text { Card }\left(\omega_{\beta}\right)<\operatorname{Card} \nVdash
$$

donc :

il existe $\mathrm{T} \in \mathcal{X}$ tel que pour tout $\nabla \in \omega_{\beta}, \nu>\lambda$, on ait $\quad r_{T}^{\prime}\left(x_{\nu}\right) \geqslant a$

Il suffit donc de choisir $T_{\lambda}=T$

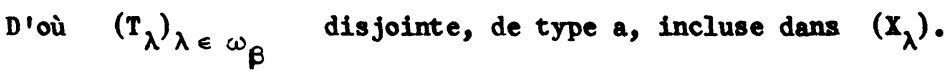

b) Si a n'a pas de prédécesseur, toute famille $\left(x_{i}\right)_{i \in I}$ telle que $r\left(x_{i}\right)=$ Card $I=a$ pour tout $i$, contient une famille disjointe de type a.

si $a=U_{0}$, ceci résulte du lemene 3 et de a) $\operatorname{car} a^{s}=2^{a}>a$.

si a $>U_{0}, a=u_{\alpha}$ où $\alpha$ n'a pas de prédécesseur, et, $\omega_{\beta}$ étant le caractère final de $\alpha \quad\left(\omega_{\beta} \leq \alpha\right)$, il existe une famille $\left(a_{\nu}\right)_{\nu} \in \omega_{\beta}$ strictement croissante de cardinaux $<a$ telle que sup a $\left[\nu \in \omega_{\beta}\right]=a$. 
146

Chapitre v

De meme il existe une famille $\left(I_{\nu}\right)_{\nu} \in \omega_{\beta}$ strictement croissante de parties de $I$ telles que Card $I_{\nu}=a_{\nu}$ pour tout $\nu$, et telle que

$$
U I_{\nu}\left[\nu \in \omega_{\beta}\right]=I \text {. }
$$

Soit en outre pour tout $\nu \in \omega_{\beta}, b_{\nu}$ le successeur de $a_{\nu} \cdot$

L'on va construire par récurrence transfinie sur $\lambda \in \omega_{\beta}$ une famille $\left(Y_{i}^{\lambda}\right) \quad\left[i \in I_{\lambda}\right]$ disjointe et telle que :

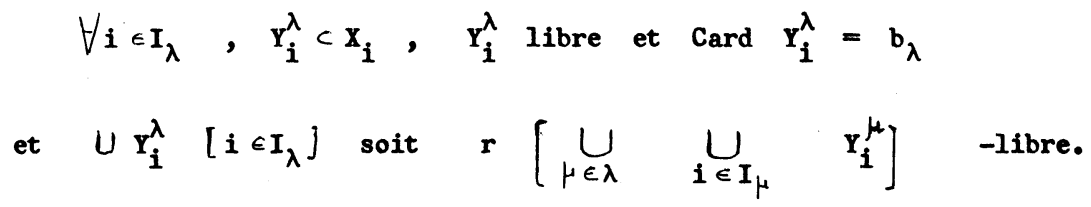

En effet supposons ces familles construires pour tout $\lambda \epsilon \mu$.

Alors :

$$
r\left(\bigcup_{\lambda \in \mu} \bigcup_{i \in I_{\mu}} \mathbf{r}_{i}^{\mu}\right) \leq \sum_{\mu \in \lambda} a_{\nu} \cdot b_{\nu}=\sum_{\mu \in \lambda} b_{\nu}<b_{\lambda}<a
$$

$$
\begin{array}{cl}
\text { donc si } & r^{\prime}=r \quad\left[\bigcup_{\mu \in \lambda} \bigcup_{i \in I_{\mu}} Y_{i}^{\mu}\right] \\
& \forall i \in I \quad, r^{\prime}\left(x_{i}\right)=r\left(x_{i}\right) \geqslant a
\end{array}
$$

Or $b_{\mu}$ est régulier et $\operatorname{Card} \mu<\operatorname{Card} \omega_{\beta} \leqslant a$, donc d'après a), il existe :

$$
\begin{aligned}
& \left(Y_{i}^{\mu}\right)_{i \in I_{\mu}} \quad \text { disjointe telle que } \\
& \forall \mathbf{i} \in \mathbf{I}_{\mu}, \quad \mathbf{y}_{\mathbf{i}}^{\mu} \subset \mathbf{x}_{\mathbf{i}}, \quad \mathbf{y}_{\mathbf{i}}^{\mu} \quad \mathbf{r}^{\prime} \text {-libre et } \quad \operatorname{Card} \mathbf{y}_{\mathbf{i}}^{\mu}=\mathbf{b}_{\mu} . \\
& \text { Donc } U \mathbf{Y}_{\mathbf{i}}^{\mu} \quad\left[i \in I_{\mu}\right] \quad \text { est } \mathbf{r}^{\prime} \text {-libre } \\
& \text { Il est clair alors que : } \\
& \left(Y_{i}^{\lambda}\right) \quad\left[(i, \lambda) \in \bigcup_{\lambda \in \omega_{\beta}} I_{\lambda} \times \lambda\right] \quad \text { est } \quad r \text {-disjointe, et pour tout } i \in I \text {, }
\end{aligned}
$$




$$
\begin{aligned}
& Y_{i}=U Y_{i}^{\lambda}\left[\lambda \in \omega_{\beta} \quad \text { et } \quad i \in I_{\lambda}\right] \\
& Y_{i} \text { est libre et Card } Y_{i}=a \text {; et on a }\left(Y_{i}\right)_{i \in I}
\end{aligned}
$$

$r$-disjointe, de type $a$, inčluse dans $\left(x_{i}\right)$

\section{Q F D}

leme 50- Soit a un cardinal infini, $\left(x_{1}\right)_{i \in I}$ une famille disjointe de parties libres d'un $\varphi$-espace $E$ telles que $r\left(x_{i}\right) \leq a$ pour tout $i$, et $X$ une partie de rang a .

si

$$
r\left[x_{I}\right](x)<a \text { et } \text { Card } I \leq a
$$

on a au moins l'une des trois situations suivantes :
(A) $\exists i \in I$ et $\exists y_{i} \subset x_{i}$ tel que $r\left[x_{I}-Y_{i}\right]$ (X) $=a$
(B) $\exists J \subset I$ tel que Card $J=-$ et $\exists\left(Y_{i}\right)_{i \in J} \subset\left(x_{i}\right)_{i \in J}$ tels que :

(1) $\forall i \in J, r\left(Y_{i}\right)<\bar{a}$; les $r\left(Y_{i}\right)$ sont distincts entre eux ; et

$$
\text { Sup } r\left(Y_{i}\right)[i \in J]=a
$$

(2)

$$
r\left[x_{I}-y_{J}\right] \quad(x)=a
$$

En outre si on tronque $J$ pour tout $b<a$ en :

$$
J_{b}=\left\{i / i \in J \text { et } r\left(Y_{i}\right)>b\right\} \text {, la famille }\left(Y_{i}\right)\left[i \in J_{b}\right]
$$

vérifie aussi les propriétés (1) et (2).

(C) $\exists j \subset I$ tel que Card $J=a$ et $\exists\left(x_{i}\right)_{i \in J} \in\left(x_{i}\right)_{i \in I}$ tel que

$$
r\left[x_{I}-\left\{x_{i} / i \in I\right\}\right] \quad(x)=a
$$

et pour tout $K \subset J$ tel que Card $(J-K)<a, \quad\left(x_{i}\right)_{i \in K}$ a ces propriétés. 
Démonstration :

L'on peut supposer que $X$ est libre.

Soit $z$ une $r[X]$-base de $x_{I}$ et $Y$ une $r\left[x_{I}\right]$-base de $X$. $X \cup Z$ et $X_{I} \cup Y$ sont deux bases de $x_{I} \cup X$, et :

$$
X \cap Z=x_{I} \cap Y=\varnothing \text {. Donc : }
$$

Card $(X \cup Z)=$ Card $\left(X_{I} \cup Y\right)$, et comme Card $Y<a$,

Card $(X-Y)=\operatorname{Card}\left(X_{I}-Z\right)=$ Card $X=a$

Toute partie c de $x_{I}-z$ est une $r\left[\left(x_{I}-c\right) \cup Z \cup Y\right]$-base de $x$. Donc :

$$
\mathbf{r}\left[X_{I}-c\right](X) \geq \mathbf{r}\left[\mathrm{Y} \cup Z \cup\left(X_{I}-c\right)\right](x)=\text { Card } c
$$

et :

Pour toute partie $c$ de $x_{I}-z$ telle que Card $c=a$, on a $\underline{\mathbf{r}}\left[\mathbf{x}_{I}=\mathbf{c}\right](\mathbf{x}) \geqslant \mathbf{a}$.

Comme Card $\left(x_{I}-z\right)=a$, on a trois cas :

10) S'il existe $i \in I$ tel que Card $\left(x_{i}-z\right)=a$, on a la situation (A).

20) Sinon, pour tout $i \in I$, Card $\left(x_{i}-z\right)<a$; mais si

$$
J=\left\{i / i \in I \text { et } x_{i}-z \neq \varnothing\right\} \text { est tel que Card } J=a,
$$

on a la situation (c) (prendre $x_{i} \in x_{i}-z \quad \ldots$ )

$\left.3^{\circ}\right)$ Enfin si : $\forall i \in I$, Card $\left(x_{i}-z\right)<a$ et Card $J<a$, alors :

$$
a=\sum_{i \in J} \text { Card }\left(x_{i}-z\right)
$$

donc a est singulier, Card $J \geqslant \bar{a}$ et $\operatorname{Sup}_{i \in J}\left(\operatorname{Card}\left(X_{i}-z\right)\right)=a$ et il est alors aisé d'expliciter la solution (B). 


\subsection{4.- Demonstration du théorème 2}

Soit $\left(x_{i}\right)_{i \in I}$ une famille de parties d'un $\varphi$-espace $E$ telle que $r\left(x_{i}\right)=a_{i}$ soit infini pour tout $i \in I$, et telle que $A=\left(a_{i}\right)_{i \in I}$ soit faiblement lisse (resp. soit lisse, en admettant l'hypothèse du continu généralise) et vérifie (C F).

Soit $\alpha=$ Ord (L) et $\nu \longrightarrow l_{\nu}$ l'isomorphisme de $\alpha$ sur L. Pour tout $\nu \in \alpha$, on note $I_{\nu}$ (resp. $J_{\nu}$ ) l'ensemble des $i \in I$ tels que $r\left(x_{1}\right)=l_{\nu}$ $\left(\right.$ resp $\left.<l_{\nu}\right)$ et $J_{\nu}^{\prime}=J_{\nu} \cup I_{\nu}$.

Coume $\mathscr{L}=\left(l_{\nu}\right)_{\nu \in \alpha}$ vérifie (C F), il existe une famille $\mathcal{M}=\left(m_{\nu}\right)_{\nu \in \alpha}, \mathscr{L}$-forte.

Nous allons construire par récurrence transfinie sur $\nu \in \alpha$ une famille $\left(z_{i}^{\nu}\right) \quad\left[i \in J_{0}^{\prime}\right]$ disjointe, où chaque $z_{i}^{\nu}$ est une partie libre de $x_{i}$ telle que :

$$
\operatorname{Card} z_{i}^{\nu}=r\left(x_{i}\right)=a_{i}=\ell_{\tau(i)}
$$

ces familles vérifiant :

$$
\forall \nu \in \alpha, \forall i \in J_{\nu},\left(z_{i}^{\lambda}\right) \tau(i) \leq \lambda \leq \nu \text { est décroissante et }
$$

Card $\left(\bigcap_{\tau(i) \leqslant \lambda<0} z_{i}^{\lambda}-z_{i}^{\nu}\right)$ est nul pour presque tout 1 , sauf Min $\left(U_{1}, m \tau(i)\right)$ au plus où il est égal à $m_{\tau(i)}$.

a) D'après le lemue 4 , il existe une famille $\left(z_{i}^{0}\right) \quad\left[i \in I_{0}\right]$ disjointe telle que, pour tout $i \in I_{0}, z_{i}^{0}$ soit une partie libre de $x_{i}$ et Card $z_{i}^{0}=\ell_{0}$

b) Supposons que l'on ait construit ces familles pour $\lambda \in \nu$

b) Alors si o n'a pas de prédécesseur : 
150

Chapitre v

$$
\forall \mathbf{i} \in J_{\nu} \quad, \quad \mathbf{T}_{\mathbf{i}}=\cap \mathbf{z}_{\mathbf{i}}^{\lambda} \quad[\tau(\mathbf{i}) \leqslant \lambda<\nu]
$$

est une partie libre de $x_{i}$ telle que :

$$
\text { Card }\left(z_{i}^{\tau(i)}-T_{i}\right) \leqslant m_{\tau(i)} \text { et Card } T_{i}=a_{i}
$$

et $\quad\left(T_{i}\right)_{i \in J}$ est disjointe.

Considérons sur $I_{\nu}$ un bon ordre de type $\beta$, naturel si $I_{\nu}$

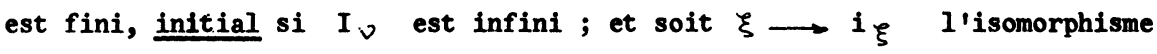
de $\beta$ sur $I_{\nu}$. Il est alors aisé de construire par récurrence transfinie sur $\xi$ une famille

$$
\left(\left(T_{i}^{\zeta}\right)_{i \in J_{\nu}}\right)_{\xi \in \beta}
$$

telle que

$$
\begin{aligned}
& \forall i \in J_{\nu} \quad, \quad\left(T_{i}^{\xi}\right)_{\xi \in \beta} \quad \text { soit décroissante. } \\
& \forall \xi \in \beta \quad, \quad \mathrm{T}_{i} \supset \mathrm{T}_{i}^{\xi} \text { et } \operatorname{Card}\left(\mathrm{T}_{i}-\mathrm{T}_{i}^{\xi}\right) \leqslant \operatorname{Card} \xi \times \mathrm{m}_{\tau(i)} \text {; } \\
& \text { et } \quad \forall \eta \in \xi+1, \quad \mathbf{r}\left[\bigcup_{\mathbf{j} \in \mathbf{J}_{\nu}} \mathbf{r}_{\mathbf{j}}^{\xi}\right] \quad\left(\mathbf{x}_{\mathbf{i}_{\eta}}\right)=l_{\nu}
\end{aligned}
$$

en utilisant le lemme 5 pour chaque $\xi$.

En outre, en prenant soin de ne diminuer plus avant $T_{i}^{\xi}$ que si Card $\xi<m_{\tau(i)}$, et meme de ne jamais diminuer $T_{i}^{\xi}$ si $i \in J^{\prime}$, , on obtient pour tout $i \in J_{\nu} \quad$ :

$$
z_{i}^{\nu}=\cap T_{i}^{\xi} \quad[\xi \in \beta]
$$

telle que : Card $\left(T_{i}-z_{i}^{\nu}\right) \leq m_{\tau(i)}$, donc Card $z_{i}^{\nu}=l_{\tau(i)}$

Alors $\left(z_{i}^{\nu}\right)_{i \in J_{\nu}}$ est disjointe, et, soit $r^{\prime}=r\left[U z_{i}^{\nu}\left[j \in J_{\nu}\right]\right]$ :

$$
\forall i \in I_{\nu}, \quad r^{\prime}\left(x_{1}\right)=l_{\nu}=a_{i} .
$$


FAMILLES DISJOINTES INCLUSES (INFINIES)

151

$\mathrm{b}_{2} \mid \underline{\mathrm{si}} \vee$ a un prédécesseur $\cup-1$ :

$$
r\left(U z_{i}^{\nu-1}\left[i \in J_{0}\right]\right)=\sum_{\lambda \in \nu} l_{\lambda} \operatorname{card} I_{\lambda}=\ell_{\nu=i}
$$

Soit $r^{\prime}=r \quad\left[U z_{i}^{\nu-1}\left[i \in J_{\nu}\right]\right]$; comme $\ell_{\nu}>\ell_{\nu-1}$, on a :

$$
\forall i \in I_{\nu} \quad, \quad r^{\prime}\left(x_{i}\right)=l_{\nu}
$$

L'on pose : $\forall i \in J_{\nu} \quad, \quad z_{i}^{\nu}=z_{i}^{\nu-1}$

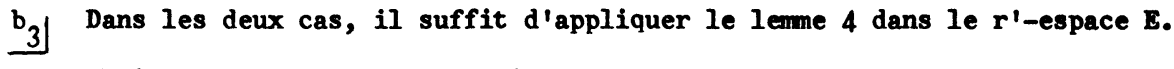

L'on obtient une famille r'disjointe

$$
\left(z_{i}^{\nu}\right)_{i \in I_{\nu}} \quad \text { telle que pour tout } i \in I_{\nu}, z_{i}^{\nu} \text { soit une }
$$

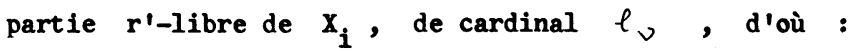

$$
\left(z_{i}^{\nu}\right) \quad\left[i \in J^{\prime}{ }_{\nu}\right]
$$

c) Construction de la solution : Soit pour tout $i \in I$

$$
z_{i}=n z_{i}^{\nu} \quad[\tau(i)<\nu<\alpha]
$$

$\left(z_{i}\right)_{i \in I}$ est disjointe et pour tout $i \in I, z_{i}$ est une partie libre de $x_{i} \cdot$ En outre, si on a choisi $\mathcal{M} \mathscr{L}$-forte vérifiant la condition du lemme 2 , on a :

$$
\forall \nu \in \alpha, \quad \text { Card }\left\{\lambda / \lambda>\nu \quad \text { et } m_{\lambda} \geqslant \ell_{\nu}\right\} \leqslant u_{1}
$$

Donc pour tout $i \in I \quad$ :

$$
\begin{aligned}
& \text { ou bien } i \in J^{\prime}, \quad \text { et } \quad z_{i}=z_{i}^{\tau(i)}: \operatorname{Card} z_{i}=a_{i} \\
& \text { ou bien } i \in I-J^{\prime}, \text { et } \\
& \text { Card }\left(z_{i}^{\tau(i)}-z_{i}\right) \leqslant U_{1} \cdot m_{\tau(i)}<a_{i} \\
& \text { donc Card } z_{i}=a_{i} \text {. }
\end{aligned}
$$


5.5. - Familles dénombrables quelconques.

On déduit des théorìmes 1 et 2 , et de la réduction de l'étude d'une famille $\left(x_{1}\right)$ quelconque les corollaires suivants :

Soit $\left(x_{i}\right)_{i \in I}$ une famille dénombrable de parties d'un $\varphi$-espace $E$, et $N$ (resp. $K$; resp. I') l'ensemble des $i \in I$ tels que $\operatorname{dim} \varphi\left(x_{i}\right)=1^{5}$ 。 $\left(\right.$ resp. $<d^{\prime}$; resp. $\left.>d_{0}^{\prime}\right)$ :

Corollatire 1.- Toute solution sur I - I' se prolonge en une solution sur $I_{2}$ 1.e. pour toute $\left(a_{i}\right)_{i \in I-I}$ et toute $\left(z_{i}\right)_{i \in I-I l} \varphi$-disjointe où $z_{i} \subset X_{i}$ et dim $\varphi\left(z_{i}\right)=a_{i}$, il existe $\left(z_{i}\right)_{i \in I} \varphi$-disjointe, incluse danss $\left(x_{i}\right)$ et telle que dim $\varphi\left(z_{i}\right)=\operatorname{dim} \varphi\left(x_{1}\right)$ pour les $i$ restant.

Corollaire 2.- Si l'une des conditions $n_{N}=\not n$ ou $n_{K}$ est fini" est réalisée, alors $\left(X_{1}\right)$ contient $\left(Y_{i}\right) \varphi$-disjointe telle que $\operatorname{dim} \varphi\left(Y_{1}\right)=\operatorname{dim} \varphi\left(X_{i}\right)$ pour tout $i \in I-K$ et $\operatorname{dim} \varphi\left(Y_{i}\right)=a_{i}$ pour $i \in K$, si et seulement si : pour tout $J$ fini, $J \subset K, \operatorname{dim} \varphi\left(x_{J}\right) \geq a_{J}$

Corolleire 3.- si Card $K=d^{4}$ et $N \neq \emptyset$, soit $\left(a_{i}\right)_{i \in K}$ telle que pour tout $J$ fini, $J \subset K$, dim $\varphi\left(x_{J}\right) \geq a_{J}$. Soit, de plus, $\left(Y_{i}\right)_{i \in K} \varphi$-disjointe où $Y_{i} \subset X_{1}$ et dim $\varphi\left(Y_{i}\right)=a_{i}$ (c.f. théorème 1).

En posant $Y=Y_{K}$ et $H$ l'ensemble des $i \in N$ tels que $\left.\operatorname{dim}_{Y} \varphi_{Y}\left(x_{K}\right)<\right\}_{0}$;

a) $\left(Y_{1}\right)_{1 \in K}$ se prolonge en une solution pour I si et seulement si $H=\varnothing$;

b) si $H \neq \phi_{2}$ il n'existe de solution sur I que si $\operatorname{dim}_{Y} \varphi_{Y}\left(x_{K}\right)=f_{0}$;

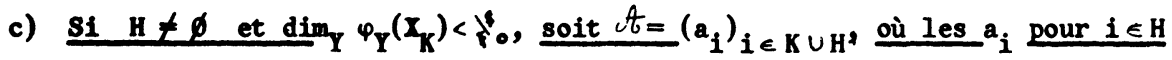
sont finis suffisamment grands [ainsi pour $a_{H}>d i m_{Y} \varphi_{Y}\left[X_{H} \cup K\right]$, alors $\left(X_{i}\right)$ est 
de type tot ne contiant aucune famille $\varphi$-dis jointe de type $t$.

Ces corollaires résolvent le cas dénombrable dans sa généralité. Il reste uniquement à étudier le cas où $I^{\prime}=\varnothing$, Card $K=l_{0}^{\prime}$ et $N \neq \varnothing$ :

$$
\left(x_{i}\right)_{i \in I} \text { est une famille de parties de }(E, \varphi) \text { telle que : }
$$

I admet une partition $\left(I^{1}, I_{1}\right) ; I^{1}$ étant dénombrable, telle que

$\operatorname{dim} \varphi\left(x_{i}\right)<i_{0}$ pour $i \in I^{1}$ et

$\operatorname{dim} \varphi\left(x_{i}\right)=s^{5}$. pour $i \in I_{1}$

choisissant une solution "maximale" $\left(Y_{i}\right)$ de $\left(x_{i}\right)_{i \in I^{1}}$,

$\varphi\left(Y_{I^{1}}\right)=\varphi\left(X_{I^{1}}\right)$ et il nous suffit d'étudier "exactement comme ${ }^{\left(X_{i}\right)_{i \in I}}$ "la

famille $\left(X_{i}\right)_{i \in I_{1}}$ dans $E$ considéré comme $\varphi_{X_{I}{ }^{1}}$-espace (on prend $\varphi_{Y_{I}{ }^{1}}$ si

$\left(Y_{i}\right) n^{\prime}$ 'est pas maximale). On se ramène de proche en proche à l'étude d'une famille $\quad\left(\left(x_{i}\right)_{i \in I_{\lambda}}\right)_{0 \leqslant \lambda<v}$ de familles de type ci-dessus ... où $v$ est un ordinal dénombrable. 
Chap. VI Un théorème d'échange; circuits

généralisés; Application a une conjecture de Rado

\section{1. - Le théorème d'échange pour un élément.}

Problème : Soit $A$ un sous $\varphi$-espace de $E, B$ une base de $A$ et $x \in A-B$. A quelle condition existe-t-il $y \in B$, et quels sont tous les $y \in B$ tels que : $B^{\prime}=B-y+x$ soit une nouvelle base de $A ;$ et en outre que deviennent les $B_{z}$ quand $z \in A$.

Voici d'abord quelques propriétés de la fonction $L_{x}$ définie au paragraphe $1.4 \cdot 3$

Soit $\mathrm{L}$ une partie libre de $(E, \varphi)$ :

a) $M$ étant une seconde partie libre et $x \in \varphi(L) \cap \varphi(M)$, on a les équivalences logiques :

$$
\left(L_{x}=M_{x}\right) \Longleftrightarrow\left(L_{x} \subset M\right) \Longleftrightarrow\left(M_{x} \subset L\right) \Longleftrightarrow(x \in \varphi(I \cap M))
$$

b) $(M, N)$ étant une partition de $L$, considérons $E$ comme $\psi$-espace où $\psi=\varphi_{N^{*}}$ On sait qu'alors $M$ est une $\Psi$-base de $\varphi(L)$, et 1 'on a :

$$
\forall z \in \varphi(L), M_{z}(\operatorname{pour} \psi)=M \cap L_{z}(\operatorname{pour} \varphi)
$$

Ces propriétés se vérifient aisément sur les circuits et en utilisant les résultats sur $\varphi_{A}$ vus en 2.1 .3 .

Théorème 1.- Soient $L$ une partie libre de $(E, \varphi), y \in L$ et $x \in \varphi(L)$, alors on a l'équivalence :

$$
\left(L^{\prime}=L-y+x \quad \text { est } \varphi-I \text { ibre }\right) \longleftrightarrow\left(y \in L_{x}\right)
$$

et en outre dans ce cas :

a) I' est une $\varphi$-base de $\varphi(L)$

b) Pour tout ze $\varphi(L)=\varphi\left(L^{\prime}\right)$, on a :

$$
\left(\mathrm{L}_{\mathrm{z}}=\mathrm{L}^{\prime}{ }_{\mathrm{z}}\right) \Longleftrightarrow\left(\mathrm{y} \& \mathrm{~L}_{\mathrm{z}}\right) \Longleftrightarrow\left(\mathrm{x} \notin \mathrm{L}_{\mathrm{z}}^{\prime}\right) \Longleftrightarrow\left(\mathrm{z} \in \varphi(\mathrm{L}-\mathrm{y})=\varphi\left(\mathrm{L}^{\prime}-\mathrm{x}\right)\right)
$$


c) Pour tout $z \in \varphi(L)$ tel que $y \in L_{z}$, on a :

$$
L_{z}^{\prime}=\left(L_{x} \cup L_{z}-K\right)+x \text { où } y \in K \text { et } K \in L_{x} \cap L_{y}
$$

L'on verra ci-dessus que ce théorème est vrai (avec la même généralité) dans les espaces vectoriels. Voici d'abord un corollaire qui résoud le problème posé ci-dessus :

Corollaire.- Soit B une base d'un sous-espace A de (E, $\varphi$ ). Etant donnés $\underline{x \in A}-\mathrm{B}$ et $y \in B$, les propriétés suivantes sont équivalentes :

(1) $-B^{\prime}=B-y+x$ est une base de $A$

(2) $-\quad y \in B_{x}$

En outre dans ce cas, pour $z \in \varphi(L)$ on a :
a) $-\left(y \notin B_{z}\right) \longleftrightarrow\left(B_{z}^{\prime}=B_{z}\right) \longleftrightarrow(z \in \varphi(B-y))$
b) $-\underline{\left(B_{z}=\{y\}\right) \longleftrightarrow\left(B_{z}^{\prime}=B_{x}-y+x\right) \longleftrightarrow(z \in \varphi(Y)-\varphi(\phi))}$
c) - Si $y \in B_{z}, B_{z}^{\prime}=\left(B_{x} \cup B_{z}-K\right)+x$ où $y \in K$ et $K \subset B_{x} \cap B_{z}$

Autrement dit, étant donné $x \in A$ et $B$ base de $A(x \notin B)$, on ne peut échanger $x$ avec un élément de $B$ pour obtenir une nouvelle base de $A$ que d'un nombre fini de manières, i.e. en choisissant un élément quelconque de $B_{x}$. Les équivalences précisent les cas où : a) $B_{z}$ est inchangé ; et b) $B_{z}$ est changé "comme dans un circuit". On retrouve en particulier le théorème 5 de 1.2.5.

Démonstration : Nous allons d'abord démontrer que $L$ ' est une $\varphi$-base de $\varphi(L)$ si et seulement si $y \in L_{x}$. En effet si $L^{\prime}$ est $\varphi$-base de $\varphi(L)$, $L^{\prime}$ est libre, donc $x \notin \varphi(L-y)$ et comme $x \in \varphi(L), y \in L_{x}$. Inversement si $y \in L_{x}, x \notin \varphi(L-y)$, et considérant $E$ comme $\psi$-espace où $\Psi=\varphi_{L_{-y}}=\varphi_{L^{\prime}-x^{\prime}}\{y\}$ est une $\psi$-base de $\varphi(L)$, donc $\{x\}$ aussi : $L-y+x$ est une $\varphi$-base de $\varphi(I)$.

Soit maintenant $x \in \varphi(L)$ et $y \in L_{x} \cdot L^{\prime}=L-y+x$ est une $\varphi$-base de $\varphi(L)$, et pour tout $z \in \varphi(L)$.

$-y \notin L_{z}$ si et seulement si $z \in \varphi(L-y)$, i.e. si et seulement si $L_{z}=L_{z}^{\prime}$. 
D'où les équivalences b) en remarquant la symétrie entre $L$ et $L^{\prime}$, sachant que $L_{y}^{\prime}=L_{x}-y+x$.

- Si $y \in \mathrm{I}_{z}$,

$\left.1^{\circ}\right)-\left(y \in L_{z}\right) \longleftrightarrow\left(x \in L_{z}^{\prime}\right)$ d'après les équivalences b)

20) - On a: $L_{z}^{\prime} \cap\left(L-L_{x}\right)=L_{z} \cap\left(L-L_{x}\right)$.

$\left.3^{\circ}\right)$ - Soit $M=I_{x}-\left(L_{x} \cap L_{z}\right)+y$ et $N=I-M$.

On a $M_{z}=M \cap L_{z}=\{y\} \quad\left(\right.$ pour $\left.\psi=\varphi_{N}\right)$, donc $\{y, z\}$ est un $\psi$-circuit (on suppose $y \neq z$, sinon c) est trivial). Or $M_{x}=M$, donc $M^{\prime}=M-y+x$ est une $\psi$-base de $\varphi(L)$. Comme $\{y, z\}$ est un $\psi$-circuit, $M_{z}^{\prime}=M_{y}^{\prime}=M^{\prime}$, donc

$$
L_{z}^{\prime} \supset M^{\prime}=\left(L_{x}-L_{x} \cap L_{z}\right)+x \cdot
$$

D'où c) : $L_{z}^{\prime}=\left(L_{x} \cup L_{z}-K\right)+x$ où $K \subset L_{x} \cap L_{z}$ et $y \in K$.

C.Q.F.D.

On ne peut espérer obtenir un résultat meilleur (i.e. préciser K) sans hypothèses supplémentaires. Nous allons le démontrer dans le cas des espaces vectoriels :

Exemple : Soient $\mathrm{L}=\left\{\ell_{0}, \ell_{1}, \ldots, \ell_{\mathrm{p}}\right\}$ une partie libre ayant p+1 éléments d'un espace vectoriel E sur un corps $k$, et :

(1) $\quad x=\sum_{i=0}^{p} \lambda_{i} \quad \ell_{i}$ où les $\lambda_{i}$ sont tous dans $k^{\prime}=k-\{0\}$

(2) $\quad y=\ell_{0} \quad$ et $z=\sum_{i=1}^{q} \quad \mu_{i} \quad \ell_{i} \quad$ où $q \leq p \quad$ et les $\mu_{i} \in k^{\prime}$

(le corps $k$, les entiers $p$ et $q$ tels que $0 \leqslant q \leqslant p$ et $\left(\lambda_{i}\right),\left(\mu_{i}\right)$ variant, on a ici le cas le plus général (à un isomorphisme près) concernant les espaces vectoriels, quand on néglige ce qui se passe en dehors de $L_{x}$ ).

Alors $\mathbf{L}_{\mathbf{x}}=\mathbf{L}, \mathrm{L}_{\mathrm{z}}=\left\{\mathrm{y}=\ell_{0}, \ell_{1}, \ldots, \ell_{\mathrm{q}}\right\}$ et $\mathrm{L}$ et $I^{\prime}=\left\{x, l_{1}, \ldots, \ell_{p}\right\} \quad$ sont deux bases du sous-espace vectoriel $F$ engendré par L. On se propose de déterminer $L^{\prime}, p, q,\left(\lambda_{i}\right)$ et $\left(\mu_{i}\right)$ étant des paramètres. 
L' étant une base de F, z s'écrit de manière unique :

(3)

$$
\begin{aligned}
& z=\nu_{0} x+\sum_{i=1}^{p} \nu_{i} \ell_{i} \text { où les } \nu_{i} \in k \\
& \text { Des relations }(1),(2),(3) \text { on tire : } \\
& z=\sum_{i=0}^{q} \mu_{i} l_{i}=\nu_{0}\left(\sum_{i=1}^{p} \lambda_{i} \ell_{i}\right)+\sum_{i=1}^{p} \nu_{i} l_{i}
\end{aligned}
$$

et, d'après l'unicité de la décomposition de $z$ en combinaison linéaire des $\ell_{i}$ :

$$
\begin{aligned}
& \begin{cases}\nu_{0} \lambda_{0}=\mu_{0} & \\
\nu_{0} \lambda_{0}+\nu_{i}=\mu_{0} & \text { pour } i=1, \ldots, q \\
\nu_{0} \lambda_{i}+\nu_{i}=0 & \text { pour } i=q+1, \ldots, p\end{cases} \\
& \text { or } \lambda_{0} \mu_{0} \neq 0 \text {, donc } \nu_{0} \neq 0 \text { et } x \in L_{z}^{\prime} \text {; et pour. } \\
& i=q+1, \ldots, p, \nu_{i}=\nu_{0} \quad \lambda_{i} \neq 0:\left\{e_{q+1}, \ldots, e_{p}\right\} \subset L_{z}^{\prime} \cdot
\end{aligned}
$$

Donc :

$$
L_{z}^{\prime}=\left\{x, e_{q+1}, \ldots, e_{p}\right\} \cup\left\{l_{i} / i \in\{1, \ldots, q\} \text { et } H_{i} \lambda_{0}-H_{0} \lambda_{i} \neq 0\right\}
$$

sachant que $\nu_{i}=\mu_{i}-\nu_{0} \lambda_{i}=\frac{1}{\lambda_{0}}\left(\mu_{i} \lambda_{0}-\mu_{0} \lambda_{i}\right)$ pour $i=1, \ldots, q$ D'où :

$$
L_{z}^{\prime}=\left(L_{x} \cup L_{z}-K\right)+x
$$

où $K=\{y\} \cup\left\{l_{i} / i \in\{1, \ldots\right.$, q $\}$ et $\left.H_{i} \lambda_{0}-H_{0} \lambda_{i}=0\right\}<L_{x} \cap L_{z}$

Mais il est bien clair que, $0 \leqslant q \leqslant p$ étant donnés, pour tout $J \subset\{1, \ldots, q\}$, il existe des couples $\left(\left(\lambda_{i}\right),\left(\mu_{i}\right)\right)$ tels que $K=J$.

En conclusion, le théorème 1 est vrai sans amélioration dans les espaces vectoriels : d'un autre point de vue, on peut dire que le théorème 1 sur les espaces vectoriels s'étend sans changement aux $\varphi$-espaces. 
6.2.- Circuits généralisés; La fonction $\mathrm{L}_{\mathrm{X}}$; Bases d'un circuit généralisé.

6.2.1.- Définitions

Définition 1.-

a) Etant donné un ensemble $\mathscr{C}$ de circuits de $(E, \varphi)$, on appelle base de $\mathscr{C}$ toute partie libre $\mathrm{L}$ de $\mathrm{E}$ telle que chacun des circuits de $\mathscr{C}$ ait au plus un élément hors de $L$.

b) On appelle circuit généralisé (on écrit c.g. en abrégé) de $(E, \varphi)$ tout ensemble $\mathscr{C}$ de circuits admettant au moins une base. $\mathscr{C}$ est dit propre si tous ses éléments ont au moins deux éléments ; sinon il est dit impropre.

$\{\{x\} / x \in \varphi(\varnothing)\}$ est un c.g., et toute partie libre $L$ de $E$ en est une base. Aussi ne considérera-t-on que des circuits ayant au moins deux éléments par la suite.

Des résultats de 1.4 .3 . on déduit que :
Tout c.g. $\mathscr{C}$ de base $\mathrm{L}$ dans $(E, \varphi)$ est de la forme :

$$
\zeta=\left\{\mathbf{l}_{\mathbf{x}}+\mathbf{x} / \mathbf{x} \in \mathbf{N}\right\}
$$

où $\mathrm{N}$ est l'ensemble des éléments des circuits de $\mathscr{C}$ qui ne sont pas dans $\mathrm{L}$. En outre l'application qui à tout circuit de $\mathscr{C}$ associe son élément en dehors de $\mathrm{L}$ (i.e. dans $N$ ) est une bijection de $\mathscr{C}$ sur $N$.

D'où la "correspondance Galoisienne" :

Théorème 2.- Soit $L$ une partie libre de $(E, \varphi)$. L'ensemble $\tau_{L}$ des c.g. de base $L$ est un sous-treillis du treillis des parties de $₹(E)$ et l'application :

$$
\mathscr{C} \longrightarrow N=\{x / \exists c \in \mathscr{C}, x \in c-L\}=\left(\bigcup_{c \in \mathscr{C}} c\right)-L
$$

est un isomorphisme (de treillis) de $\varepsilon_{L} \operatorname{sur} ₹(\varphi(L)-L)$, les circuits générali- 
Sés propres correspondant aux parties de $\varphi(L)-(L \cup \varphi(\varnothing))$.

La correspondance inverse est :

$$
\mathrm{N} \longrightarrow \mathscr{C}=\left\{\mathrm{L}_{\mathrm{x}}+\mathrm{x} / \mathrm{x} \in \mathrm{N}\right\}
$$

\subsection{2.- La fonction $\mathrm{L}_{\mathrm{X}}$.}

Voici maintenant un théorème qui précise les relations entre $\mathscr{C}$ et $\mathrm{N}$. Il se présente comme une extension des propriétés de la fonction $I_{x}$.

Théorème 3.- Soit I une partie libre de $(E, \varphi)$. Pour tout $A \subset \varphi(L)$, il existe une partie libre minimale $L(A)$ et une seule de $L$ telle que $A \subset \varphi(L(A))$. Cette application $X \rightarrow L(X)$ de $\beta(\varphi(L))$ dans $\beta(L)$ est aussi définie par :

$$
L(A)=\cap L^{\prime} \quad\left[L^{\prime} \subset L \quad \text { et } \quad A \subset \varphi\left(L^{\prime}\right)\right]
$$

et

$$
L(A)=L_{A}=U L_{x} \quad[x \in A]
$$

$x \rightarrow I_{x}$ est un homomorphisme de treillis de parties, et en particulier pour toute famille $\left(A_{i}\right)$ de parties de $\varphi(L), L\left(\cap A_{i}\right)=\cap L\left(A_{i}\right)$ et $L\left(\cup A_{i}\right)=\cup L\left(A_{i}\right)$; mais ce n'est un isomorphisme que si $\varphi(L)=L$.

$$
\text { L'existence de } L(A) \text { définie par (1) résulte de 1.4.3. }
$$$$
\text { Alors } \forall x \in A, \quad x \in \varphi(A)<\varphi(L(A)) \text {, donc } L_{x} \in L(A) \text { et } L_{A} \subset L(A) \text {; }
$$

inversement $A \subset \varphi\left(L_{A}\right)$, donc $L(A) \subset L_{A}$; d'où l'égalité (2). Le reste du théorème 3 résulte alors aisément de (2).

$$
\text { Comme d'après (1), } L(A)=L(\varphi(A)) \text {, on a le : }
$$

(*) - On utilisera indifféremment $L_{A}$ ou $L(A)$, surtout pour éviter les indices de second ordre. 
Corollaire 1.- Pour tout $A \subset \varphi(L), L_{A}=L \varphi(A)$. En outre, si A et B sont deux parties de $\varphi(L)$, on a :

$$
\begin{aligned}
& \varphi(\mathrm{A}) \subset \varphi(\mathrm{B}) \Longrightarrow \mathrm{L}(\mathrm{A}) \subset \mathrm{L}(\mathrm{B}) \\
& \text { et } \quad \varphi(\mathrm{A})=\varphi(\mathrm{B}) \quad \Longrightarrow \quad \mathrm{L}(\mathrm{A})=\mathrm{L}(\mathrm{B})
\end{aligned}
$$

mais les réciproques sont fausses.

Soit $B$ une $\varphi$-base de $\varphi(A), L_{B}=L_{A}$, et comme $A \subset \varphi\left(L_{A}\right)$, $\operatorname{dim} \varphi(A) \leq \operatorname{dim} \varphi\left(L_{A}\right)=\operatorname{card} L_{A}$.

Si $A \subset \varphi(\varnothing), \quad L_{A}=\varnothing$ et $\operatorname{dim} \varphi(A)=\operatorname{card} L_{A}=0$

Si $\operatorname{dim} \varphi(A)=$ card $B$ est infinie :

Card $L_{A}=\operatorname{Card} L_{B} \leqslant \sum \operatorname{Card} L_{x}[x \in B]=\operatorname{Card} B$

donc dim $\varphi(A)=$ Card $L_{A^{*}}$ D'où le :

Corollaire 2.- Pour tout $A \subset \varphi(L), \underline{\operatorname{Card} L(A) \geq \operatorname{dim} \varphi(A)}$ (3) .

$\underline{L}(\mathrm{~A})$ est fini si et seulement si dim $\varphi(A)$ l'est, et on a l'égalité dans (3) quand dim $\varphi(A)$ est nulle ou infinie.

Notons qu'en général l'inégallté est stricte dans (3) quand dim $\varphi(A)$ est finie non nulle.

Corollaire 3.- Soit $I$ une partie libre de $(E, \varphi)$ et $A \subset \varphi(L)$.

a) $M$ étant une seconde partie libre telle que $A \subset \varphi(M)$, on a :

$$
\left(L_{A}=M_{A}\right) \Longleftrightarrow\left(L_{A} \subset M\right) \Longleftrightarrow\left(M_{A} \subset L\right) \longleftrightarrow(A \subset \varphi(L \cap M))
$$

b) ( $M, N)$ étant une partition de $L$ et considérant $E$ comme $\psi$-espace où $\psi=\varphi_{N}$, $M$ est une $\psi$-base de. $\varphi(L)$ et l'on a :

$$
M_{A}(\operatorname{pour} \psi)=M \cap L_{A}(\operatorname{pour} \varphi)
$$




\subsection{3.- Bases d'un circuit généralisé.}

Jusqu'ici $\mathrm{L}$ est fixé et on étudie les $\mathscr{C}$ admettant $\mathrm{L}$ comme base. On se propose maintenant d'étudier le problème suivant :

Etant donné un ensemble $\mathscr{C}$ de circuits de $E$, quelles sont toutes les bases de $\mathscr{C}$, et en particulier à quelle condition $\mathscr{C}$ est-il un c.g. ?

On suppose toujours $\mathscr{C}$ propre

On déduit aisément des définitions ci-dessus le :

Lemme 1.- Soit $\mathscr{C}$ un ensemble de circuits de $(E, \varphi)$. Si L est une base de $\mathscr{C}$, L $\cap \bar{b}$ (où $\overline{\mathscr{C}}=U \mathrm{C}[\mathrm{c} \in \mathscr{C}]$ ) est une base minimale de $\mathscr{C}$. Inversement toute base $L_{0}$ de $\mathscr{C}$ incluse dans $\overline{\mathscr{C}}$ est une base minimale de $\mathscr{C}$ et toute partie libre L contenant $L_{0}$ est une base de $\mathscr{C}$.

Il nous suffit donc d'étudier les bases de $\mathscr{C}$ incluses dans $\overline{\mathscr{C}}$. On démontre alors le :

Lemme 2.-
$\mathscr{C}=\left\{c_{1}, \ldots, c_{n}\right\} \cdot$ Les propriétés suivantes sont équivalentes :

a) $\mathscr{C}$ est un circuit généralisé

b) pour $k=2, \ldots, p, \operatorname{dim} \varphi\left(\bigcup_{i=1}^{k} c_{i}\right)=\operatorname{Card}\left(\bigcup_{i=1}^{k} c_{i}\right)-k$

b') " " " "

c) $\forall \mathrm{J} \subset\{1, \ldots, n\}, \operatorname{dim} \varphi\left(c_{J}\right)=\operatorname{Card} c_{J}-k$

c') $\forall \mathrm{J} \subset\{1, \ldots, n\}, \operatorname{dim} \varphi\left(c_{J}\right) \geq \operatorname{Card} c_{J}-k$

et dans ce cas l'ensemble des bases de $\mathscr{B}$ incluses dans $\overline{\mathscr{C}}$ est

$$
\begin{aligned}
& \beta=\left\{\bar{C}-\left\{e_{1}, \ldots, e_{n}\right\} / \text { pour } i=1, \ldots, n, l_{i} \in c_{i}-\bigcup_{j} c_{j}\right\} \neq \varnothing \\
& \bar{C}=c_{1} \cup \ldots \cup c_{n} .
\end{aligned}
$$


a) $\Rightarrow$ c). Car si $\mathscr{C}$ est un c.g., soit $I$ une base de $\mathscr{C}$, incluse dans $\overline{\mathscr{C}}$. Or $c_{i} \rightarrow \ell_{i} \in c_{i}-L$ est une bijection de $\mathscr{C}$ sur $N=\bar{C}_{-} L$, donc pour $i=1, \ldots, n$ et pour tout $j \neq i, \ell_{i} \notin c_{j}$, et $L=\bar{C}_{-}\left\{\ell_{i}, \ldots, \ell_{n}\right\} \in \beta$. De plüs, $\forall \mathrm{J} \subset\{1, \ldots, \mathrm{n}\}=\mathrm{I}$

$\mathrm{L}_{\mathrm{J}}=\bigcup_{i \in J} \mathrm{~L}_{\ell_{i}}=\mathrm{c}_{\mathrm{J}}-\left\{\ell_{i} / \mathrm{i} \in \mathrm{J}\right\}$ est une $\varphi$-base de $\varphi\left(\mathrm{c}_{\mathrm{J}}\right) ;$ d'où $\left.\mathrm{c}\right)$.

Inversement, si $L \in \oiint_{b}$, I $\varphi$-engendre $\varphi(\bar{\zeta})$ et

Card $L=\operatorname{Card} C_{I}-\operatorname{Card} I=\operatorname{dim} \varphi\left(C_{I}\right)$, donc $L$ est une $\varphi$-base de $\varphi\left(C_{I}\right): L$ est une base de $\mathscr{C}$. D'où la fin du lemme 2 .

c) $\Rightarrow$ b) : il suffit de prendre $J=\{1, \ldots, k\}$ où $k=2, \ldots, p$. Il est clair que b) $\left.\rightarrow b^{\prime}\right), \quad$ c) $\rightarrow c^{\prime}$ ) et $\left.\quad c^{\prime}\right) \rightarrow b^{\prime}$ ).

$b^{\prime} \rightarrow$ a) se démontre par récurrence sur l'entier $n$.

Ceci est trivial si $n=1$.

Supposons la propriété vraie jusqu'à $n-1$ et soit

$\mathscr{C}=\left\{c_{1}, \ldots, c_{n}\right\} \quad$ vérifiant $\left.b^{\prime}\right) . \quad b^{\prime}=\left\{c_{1}, \ldots, c_{n-1}\right\}$ vérifie aussi $\left.b^{\prime}\right)$, donc $\zeta^{\prime}$ est un c.g., et $\zeta^{\prime}=\left\{L^{\prime} l_{i}+\ell_{i} / l_{i} \in N^{\prime}\right\}$ où $L^{\prime}$ est une base de $\ell^{\prime}$ incluse dans $\bar{b}^{\prime}$ et $N^{\prime}=\bar{\zeta}^{\prime}-L^{\prime}=\left\{l_{1}, \ldots, \ell_{n-1}\right\}$.

Comme :

$\operatorname{dim} \varphi(\bar{b}) \geq \operatorname{Card} \bar{\zeta}-n=\operatorname{Card}\left(\bar{b}-\bar{b}^{\prime}\right)+\left(\operatorname{Card} \bar{b}^{\prime}-n+1\right)-1$,

$\operatorname{dim} \varphi(\bar{\zeta}) \geqslant \operatorname{dim} \varphi\left(\bar{\zeta}^{\prime}\right)+\left[\operatorname{Card}\left(\bar{\zeta}-\bar{\zeta}^{\prime}\right)-1\right]$

et comme $\bar{b}^{\prime} \subset \bar{b}$, Card $\left(\bar{b}-\bar{b}^{\prime}\right) \geq 1: \bar{b} \geqslant \bar{b}^{\prime}$. Soit alors $\ell_{n} \in c_{n}-\bar{b}^{\prime}$,

et $L=L^{\prime} \cup\left(c_{n}-l_{n}\right): L$ est une base de $\ell$, d'où a).

Nota : La condition $\operatorname{dim} \varphi\left(\bigcup_{i=1}^{n} c_{i}\right)=\operatorname{card} \bigcup_{i=1}^{n} \quad c_{i}-n \quad$ n'est pas suffisante en général

(quand $n \geq 3$ ) pour que $\zeta$ vérifie a). 
Théorème 4.- Soit $\mathscr{C}=\left(C_{i}\right)_{i \in I}$ une famille de circuits de $(E, \varphi)$ $\left(i \longrightarrow C_{i}\right.$ étant une $\underline{\text { injection }}$. Les propriétés suivantes sont équivalentes:

a) - $C$ est un circuit généralisé

b) $-\exists\left(\ell_{i}\right)_{i \in I}$ telle que : $\forall i \in I, \ell_{i} \in c_{i}-c_{I-i}$, et $\bar{b}-\left\{l_{i} / i \in I\right\}$ est libre

c) $-\forall K \in \mathcal{F}(\mathrm{I}), \operatorname{dim} \varphi\left(\mathrm{c}_{K}\right)=\operatorname{Card} \mathrm{c}_{K}$ - Card $\mathrm{K}$

c') $-\forall K \in \mathcal{F}(\mathrm{I}), \operatorname{dim} \varphi\left(\mathrm{C}_{K}\right) \geqslant \operatorname{Card} \mathrm{C}_{K}-\operatorname{Card} K$ où on note pour tout $K \in I, c_{K}=\cup c_{i}[i \in K]$ et $\overline{\mathscr{C}}=c_{I}$.

En outre quand l'une de ces propriétés est vérifiée (donc toutes) 1 'ensemble des bases de $\mathscr{C}$ incluse dans $\overline{\mathscr{C}}$ est :

$$
B=\left\{\bar{C}-\left\{\ell_{i} / i \in I\right\} \quad \forall \forall i \in I, \ell_{i} \in c_{i}-c_{I-i}\right\} \neq \varnothing .
$$

Une partie libre $\mathrm{L}$ est une base de $\mathscr{C}$ si et seulement si $\mathrm{L} \cap \bar{\zeta}$ en est une. En particulier toute base de $\mathscr{C}$ contient :

$$
B_{0}=U\left(C_{i} \cap C_{j}\right) \quad\left[\{i, j\} \in F_{2}(I)\right]
$$

Si $\mathscr{C}$ est un c.g., tout $\mathscr{C}^{\prime} \subset \mathscr{C}$ est aussi un circuit généralisé (prendre la meme base), en particulier les $\mathscr{C}^{\prime}$ finis ; d'où d'après le leme 2, a) $\Longrightarrow$ c), a) $\Longrightarrow c^{\prime}$ ) et $\left.c^{\prime}\right) \Longrightarrow$ c). On démontre comme au lemme 2 que a) $\Rightarrow$ b) et que toute base de $\mathscr{C}$, incluse dans $\overline{\mathscr{b}}$, appartient à $\beta$.

$$
\text { c) } \Longrightarrow \text { a). D'après } c) \text {, pour tout } K \in \mathcal{J}(I), \quad \varphi^{K}=\left\{c_{i} / i \in K\right\} \text { possède }
$$
la propriété c) du lemme 2 , donc $\zeta^{K}$ est un c.g. et pour tout $i \in K$, il existe $\ell_{i} \in \mathrm{C}_{i}-\mathrm{C}_{\mathrm{K}-\mathrm{i}}$. Or pour tout $i \in \mathrm{I}, \mathrm{c}_{\mathbf{i}}$ est fini, done il existe $\ell_{i} \in \mathrm{C}_{\mathbf{i}}-\mathrm{C}_{\mathrm{I}-\mathrm{i}}$, et $L=\bar{b}-\left\{l_{i} / i \in I\right\} \in B$ est une base de $\mathscr{C}$. En effet pour tout $M \in \mathcal{F}_{(L)}$, il existe $K \in \mathscr{F}(I)$ tel que $M \subset C_{K}-\left\{l_{i} / i \in K\right\}$. Or $c_{K}-\left\{l_{i} / i \in K\right\}$ est une base de $\zeta^{K}$, donc libre, et à fortiori $M$ aussi : L est $\varphi$-libre, donc base de $\mathscr{C}$. D'où a) et le reste du théorème 4 (en utilisant aussi le lemme 1). 
On a démontré en particulier le :

Corollaire.- La propriété "C est un circuit généralisé" est de caractère fini, i.e. $\mathscr{C}$ est un circuit généralisé si et seulement si tout $\mathscr{C}^{\prime} \in \mathcal{F}(\mathscr{C})$ en est un.

\subsection{4.- Propriétés caractéristiques des circuits généralisés possibles. Appli- cations.}

La notion de circuit généralisé admet pour cas particulier celle de "système fondamental de circuits" de H. Whitney dans [11] . Voici comment sont faits les c.g. quand $\varphi$ varie :

Soit $(E, \varphi), \underline{C}=\left\{B_{x}+x / x \in E-B\right\}$ où $B$ est une $\varphi$-base de $E$ est un $\varphi$-circuit généralisé maximal $\left(\varphi-c_{. g . m .}\right)$, et cette propriété est en fàit

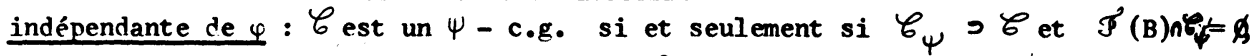
et dans ce cas B est une $\psi$-base de $E, \mathscr{C}$ un $\psi$-c.g.m., et tout $\psi$-circuit $x \notin \mathscr{C}$ est te] que Card $(X-B) \geq 2$.

La donnée d'un $\varphi$ - c.g.m. $G$ fournit déjà de bons renseignants sur la structure $(E, \varphi)$; mais en général il y a de nombreuses structures $(E, \psi)$ telles que $\mathscr{C}$ soit un $\psi$ - c.g.m. Il est intéressant d'essayer de construire une $\psi$ canoniquement associée à $\mathscr{C}$.

Mieux, la donnée de $\mathscr{C}$ est en fait indépendante de $\varphi$ : étant donné un ensemble $E$, on dit que $\mathscr{C}$ est un.c.g.p. (c.g. possible ou en puissance) de E si $\mathscr{C}$ est un ensemble de parties finies de $\mathbf{E}$ de la forme : $\mathscr{b}=\left\{B_{x}+x / \exists B \subset E, x \in E-B\right.$ et $\left(B_{x}\right)_{x \in E-B}$ famille de parties finies de $\left.B\right\}$

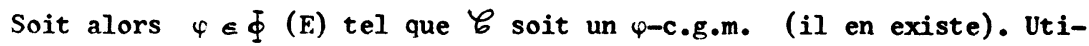
lisant $I^{\prime}$ axiome $\left(C^{\prime \prime}\right)$ des circuits, si $x, y \in E-B$ sont tels que $B_{x} \cap B_{y} \neq \varnothing$, $\forall z \in B_{x} \cap B_{y}, \quad\left(B_{x} \cup B_{y}-z\right) \cup\{x, y\}$ est réunion de circuits, d'où en particulier un ou plusieurs circuits $C$ tels que $:\{x, y\} \in C$ et $C \subset\left(B_{x} \cup B_{y}-z\right) \cup\{x, y\}$. On peut très bien vérifier $\left(C_{2}\right)$ en n'introduisant qu'un circuit $\left(B_{x}{ }^{\circ} B_{y}\right) \cup\{x, y\}$. 
D'une manière générale, posons :

$$
\mathscr{C}_{0}=\left\{\mathrm{C} / \mathrm{C} \in \mathcal{F}(\mathrm{E}) \text { et } \exists \mathrm{X} \in \mathcal{F}(E-B) \text { tel que } \mathrm{C}=\mathrm{X} \cup\left(\Sigma^{1} \mathrm{~B} \times \underline{[\mathrm{x} \in \mathrm{X}])\}}\right.\right.
$$

où $\Sigma^{1^{1}} B_{x}[x \in X]$ est $l$ 'ensemble des $z$ appartenant à un nombre impair de $B_{x}$ quand $x$ décrit $x_{0}$ Alors $\mathscr{C}_{0}$ vérifie $\left(c_{1}\right)$ et $\left(c_{2}\right)$, et définit sur $E$ une structure de O-espace tel que $\mathscr{C}$ soit un $\theta-c . g . m$.

Voici une autre manière de construire $\theta$ canoniquement :

Soit $\mathscr{C}$ un c. g. p. sur $\mathrm{E}$ et B une base de $\mathscr{C}$. $\sigma$ étant la bijection $x \underset{(B)}{\longrightarrow} \ell_{x}$ de $B$ sur $l a$ base canonique $\left(\ell_{x}\right)_{x \in B}$ de $l$ 'espace vectoriel libre $k$ (B) engendré par $B$ sur $k$, on prolonge $\sigma$ en une application (non injective en général) $f$ de $E$ dans $k^{(B)}$ telle que :

$$
x \in E-B \longrightarrow f(x)=\Sigma \lambda_{x}^{y} e_{y} \quad\left[y \in B={ }_{x}\right]
$$

où $\forall x \in E-B, \forall y \in B_{x}, \lambda_{x}^{y_{1}} \in k-\{0\}$ (prendre les $\lambda_{x}^{y}=1$ par exemple). $\sigma(\mathscr{C})$ est un $\varphi_{0}-c_{0} g_{0} m_{0}$ du $\varphi_{0}$-espace $k^{(B)}$ associé à la structure d'espace vectoriel, et l'image réciproque $\varphi$ de $\varphi_{0} \underline{\text { par } f}$ est alors telle que $\mathscr{C}$ soit un $\varphi-c . g . m$. .

Quand $k$ est grand, on a beaucoup de manières de choisir $f$, d'où en général beaucoup de $\varphi \in \Phi(E)$, représentables (pour une extension de $k$ ), telles que $\zeta$ soit un $\varphi-c . g . m$. ; mais si $k=F_{2}$ n'a que deux éléments $\underline{f}$ est entièrement déterminée, d'où canoniquement la structure de $\theta$-espace sur $E$.

Remarque 1.- Quand on considère les c. g. p. $\mathscr{C}$ sur $E$ tels que $x \rightarrow{ }^{B}$ soit une injection de $E$ dans $\mathcal{F}^{(B)} \quad$ (y compris $B_{x}=\{x\}$ si $x \in B$ ), on obtient ainsi toutes les $\varphi \in \Phi(E)$ qui sont $F_{2}$-représentables. On constate donc que la propriété pour $\mathscr{C}_{\varphi}$ :

$$
\forall\left\{x_{1}, \ldots, x_{n}\right\} \quad \varphi \text { - c.g., } \quad \Sigma^{1} x_{i}[i=1, \ldots, n] \quad \text { est un } \varphi \text {-circuit, }
$$

caractérise les $\varphi \quad{ }_{2}$-représentables, et cette propriété est vraie si et seulement si elle l'est pour toute partie finie d'un $\varphi-$ c.g. m. $C$ tel que $x \rightarrow{ }^{B} x$ soit injective.

D'une manière générale les c. g. p. $\mathscr{C}$ sur E définissent canoniquement $(E, \theta)$ k-représentable où $k$ est un corps de caractéristique 2 ayant un cardianl suffisamment grand. 
On améliore ainsi le résultat de $\mathrm{H}$. Whitney [11] appendice.

Remarque 2.- Des procédés comme ceux du type (1) permettent de transformer le problème de k-représentation de $(E, \varphi)$ en l'étude d'un système d'équations fau sens le plus général, i.e. $f \neq 0, f=0, f_{1}$ ou $f_{2}=0 \ldots$ ) ayant en général plus d'inconnues (les $\lambda_{x}^{y}$ ) que d'équations. On sait cependant que de tels systèmes n'ont pas toujours de solution.

\section{3.- Un algorithme dour résoudre une conjecture de Rado}

Dans une communication au Congrès de Stockolm (1962), M. Rado a présenté un théorème sur les espaces vectoriels, théorème dont la démonstration $n$ 'a pu jusqu'ici s'étendre aux " $\varphi$-espaces" (i.e. aux "matroids" au sens de H.Whitney, c. f. [11]) . M. Rado conjecturait alors l'une des deux éventualités suivantes : ou bien ce théorème n'est pas vrai sur les $\varphi$-espaces, et sa validité constitue un axiome supplémentaire qui nous rapproche des axiomes des $\varphi$-espaces représentables dans un espace vectoriel, ou bien on peut étendre ce théorème aux $\varphi$-espaces.

Le but de ce paragraphe est de lever définitivement ce doute en démontrant le (*) :

Théorème 5.- Soit $E$ un $\varphi$-espace, pour qu'une partie $S$ de $E$ admette une partition formée de $k$ parties $\varphi$-libres, où $k$ est un entier $>0$, il faut et il suffit que : $S$ vérifie l'une des propriétés équivalentes :

$$
\begin{aligned}
& \left(P_{k}\right): \text { Pour tout } x \in G^{\prime}(S),|X| \leq k . r(X) \text { et }|S| \geq k \\
& \left(P{ }_{k}^{\prime}\right): \text { Pour tout sous-espace } A, \quad|A \cap S| \leq k \cdot r \quad(A \cap S) \text { et }|S| \geqslant k
\end{aligned}
$$

où $\mathbf{r}$ est le rang sur $E$ associé à $\varphi$.

(*) - Après cette communication, M. R. Rado a également trouvé un raisonnement sur les espaces vectoriels qui s'étend aux "matroids". 
Les conditions $\left(P_{k}\right)$ et $\left(P_{k}\right)$ sont équivalentes. En effet :

$$
\left(P_{k}\right) \Longrightarrow\left(P_{k}\right) \quad \text { trivialement }
$$

inversement si $\left(P_{k}\right)$ est vérifiée, pour tout $x \in \mathcal{F}^{\prime}(s)$, d'où $\left(\mathbf{P}_{\mathbf{k}}\right)$

$$
|x| \leq|\varphi(x) \cap s| \quad k \cdot r(\varphi(x) \cap s)=\operatorname{kor}(x)
$$

La condition $\left(P_{k}\right)$ est nécessaire car, si $\left(S_{i}\right)_{i}=1, \ldots, k$ est une partition de $s$ en $k>0$ parties libres $s_{i}$, pour tout $x \in \mathcal{F}(s)$,

$$
|x|=\sum\left|x \cap s_{i}\right| \quad[i=1, \ldots, k] \leqslant k . \quad\left(\operatorname{Max}\left|x \cap s_{i}\right| \quad[i=1, \ldots, k]\right) .
$$

et comme $r(x) \geq\left|X \cap s_{i}\right|$ pour tout $i \quad\left(\operatorname{car} s_{i}\right.$ est $\varphi-1$ libre $),|x| \leq k r(x)$.

La difficulté essentielle est la réciproque.

Comme pour le problème de disjonction, la démonstration que nous allons donner est essentiellement constructive.

La réćiproque est connue quand $k=1$, car $s$ est libre si et seulement si : $\forall x \in T^{f}(s),|x| \leq r(x) \quad$ (alors $|x|=r(x)$ ).

Nous supposons donc désormais $k \geq 2$, et démontrons le :

Lemme 3.- Soit $S$ une partie finie d'un $\varphi$-espace $E$ et $k$ un entier 22 . SI $S$ vérifie $\left(P_{k}\right)$, pour toute famille $\left(x^{i}\right)_{i \in I}$ de parties libres $x_{i}$ telle que $y x^{i} \quad[i \in I] \leqslant s(*)$ et $I=\{1, \ldots, k\}$, il existe une famille $\left(r^{i}\right)$ i $\in I$ de parties $\varphi$-libres $\mathrm{Y}^{i}$ telle que :

$$
\underline{\underline{u}} \mathrm{X}_{\mathbf{i}}[\mathrm{i} \in \mathrm{I}] \subsetneq \underline{\mathrm{U}} \mathrm{Y}_{\mathbf{i}}[\mathrm{i} \in \mathrm{I}] \subset \mathrm{S} \quad \text { et } \quad \forall \mathrm{i} \in \mathrm{I} \quad \varphi\left(\mathrm{X}^{\mathbf{i}}\right) \subset \varphi\left(\mathrm{Y}^{\mathbf{i}}\right)
$$

(*) - Pour une famille $\left(x_{i}\right)_{i \in I}$ de parties de $E$, le terme $\underline{U} x_{i}[i \in I]$ désigne $U x_{i}[i \in I]$ et implique que les $x_{i}$ sont disjoints deux à deux. 
En itérant $|S|$-fois au plus le procédé du lemme 3 à partir de $(\phi)$, on obtient la réciproque du théorème 5 quand $S$ est fini.

Voici comment on construit $\left(Y^{i}\right)$ à partir de $\left(x^{i}\right)$ :

Soit $S \subset E, k \geqslant 2, I=\{1,2, \ldots, k\}$ et $\left(x^{i}\right){ }_{i \in I}$ une famille de parties libres de $E$ telle que $U x^{i}[i \in I] S S$.

Posant $S_{0}=S-\left(U X^{i}[i \in I]\right)=A_{0}$. S'il existe $i_{0} \in I$ tel que $S_{\circ} \notin \varphi\left(x^{i_{\bullet}}\right)$, pour tout $x_{\bullet} \in S_{\odot}-\varphi\left(x^{i_{\circ}}\right)$, la famille $\left(Y^{j}\right)_{j \in I}$ où $Y^{j}=x^{j}$ si $j \in I-i_{0}$ et $Y^{i_{0}}=X^{i_{0}}+x_{0}$ répond au lemme 1 sans autres hypothèses sur $S$ et les $x^{i}$. Il ne reste donc à étudier que le cas où :

$$
\left(a_{0}\right): s_{0} \subset A \text { où } A=n_{\varphi}\left(x^{i}\right)[i \in I]
$$

Soit alors pour tout $i \in I, A_{i}=x^{i}\left(S_{0}\right)$. S'il existe $i_{1} \in I$ tel que $A_{i_{1}} \notin A$, soit $i_{0} \in I \quad\left(i_{0} \neq i_{1}\right.$ nécessairement $)$ tel que $A_{i_{1}} \notin \varphi\left(x_{i_{0}}\right)$ :

$$
\forall x_{1} \in A_{i_{1}}-\varphi\left(x^{i_{0}}\right), \exists x_{0} \in S_{0} \text { tel que } x_{1} \in x_{x_{0}}^{i_{1}}
$$

donc $y^{i}=x_{x_{0}}^{i}-x_{1}+x_{0}$ est $\varphi$-libre (théorème 1),

$$
\mathrm{y}^{\mathbf{i}_{0}}=\mathrm{x}^{i_{0}}+\mathrm{x}_{1} \text { est } \varphi \text {-libre }\left(\mathrm{x}_{1} \notin \varphi\left(\mathrm{x}^{\mathbf{i}_{0}}\right) \text {, et posant } \mathrm{y}^{\mathbf{j}}=\mathrm{x}^{\mathbf{j}}\right. \text { pour }
$$

$j \in I-\left\{i_{0}, i_{1}\right\}$, on a $\left(Y^{j}\right)$. Il ne reste donc à étudier que le cas où $\left(a_{0}\right)$ et

$$
\left(a_{1}\right): \forall i \in I, A_{i}=x^{i}\left(s_{0}\right) \subset A
$$

D'une manière générale, pour tout entier $q \geq 0$, soit $I_{q} I$ 'ensemble des $\alpha=\left(i_{1}, \ldots, i_{q}\right) \in I^{q}$ tels que $i_{k} \neq i_{k+1}$ pour $k=1, \ldots, q-1$, donc $I_{0}=\{0\}$ et $I_{1}=I ;$ et pour tout $\alpha=\left(i_{1}, \ldots, i_{q}\right) \in I_{q}$, et tout entier $k$ tel que $0 \leqslant k \leqslant q$, soit $\alpha^{k}=\left(i_{1}, \ldots, i_{k}\right)$ et $\alpha^{0}=0$.

Supposons alors que $S$ et $\left(X^{i}\right)$ vérifient : pour $q=0,1, \ldots, p-1$, on $a(*)$

(*) Il s'agit ici de composition de fonctions $L_{X}$. On écrira de même par la suite $\alpha(k)=\alpha^{k} \ldots i(k)=i_{k}$ quand aucune confusion ne sera à craindre. 
PARTITIONS EN PARTIES LIBRES

169

$$
\left(a_{q}\right): \forall \alpha=\left(i_{q}, \ldots, i_{q}\right) \in I_{q}, A_{\alpha}=x^{i_{q}} \circ x^{i_{q}-1} \ldots \ldots . x^{i_{1}}\left(s_{0}\right) \subset A
$$

où $A=\cap_{\varphi}\left(x_{i}\right) \quad[i \in I]$.

En vertu des hypothèses successives $\left(a_{0}\right),\left(a_{1}\right) \ldots\left(a_{p-1}\right)$

${ }_{\alpha}$ est défini par récurrence ainsi :

$$
\begin{aligned}
& \quad \text { pour } q=0,1, \ldots, p-1 ; \text { pour } k=1, \ldots, q \text { et } \forall a \in I_{q} \text { : } \\
& A_{0}=s_{0} \text { et } A_{\alpha} k=x^{i_{k}}\left(A_{\alpha} k-1\right) .
\end{aligned}
$$

Alors soit pour tout $i \in I$, et pour tout entier $q, 0 \leqslant q \leqslant p$ :

$$
A_{i}^{q}=U A_{\alpha} \quad\left[\alpha=\left(i_{1}, \ldots, i_{q-1}, i\right) \in I_{q}\right] \quad\left(A_{i}^{0}=A_{0}\right)
$$

et $A^{q}=U A_{i}^{q} \quad[i \in I]=U A_{\alpha} \quad\left[\alpha \in I_{q}\right]$

Nous allons démontrer que :

$$
A^{1} \varsubsetneqq A^{2} \subsetneq \cdots \subsetneq A^{p-1} \subsetneq A^{p}
$$

10) Démontrons que $A^{1} \subset A^{2} \subset \ldots \subset A^{p}$. Ceci résulte du fäit que, pour tout i $\in I$,

$$
A_{i}^{1} \subset A_{i}^{2} \subset \ldots \subset A_{i}^{p}
$$

En effet en vertu du théorème 3 et de son corollaire 2, pour tout $(j, i) \in I_{2}, x^{i} \circ x^{j}\left(s_{0}\right) \supset x^{i}\left(s_{0}\right)$, donc $A_{i}^{1} \subset A_{i}^{2}$.

D'une manière générale, $A_{i}^{q} \subset A_{i}^{q+1}$ car pour tout $\alpha=\left(i_{1}, \ldots, i_{q-1}, i\right) \in I_{q}$, soit $f=\left(i_{1}, \ldots, i_{q-1}, j, i\right) \in I_{q+1}$ tel que $i \neq j \neq i_{q-1} \neq i$ on a ${ }_{\alpha}^{A} \subset A_{F}$ quand $I$ a trois éléments au moins. Quand I n'a que deux éléments : $I=\{1,2\}, I_{q}$ n'a que les deux éléments $\alpha_{q}=(1,2,1, \ldots)$ et $f_{q}=(2,1,2, \ldots)$ et il est alors aisé de démontrer par récurrence sur $q$ 
que : $\quad A_{\alpha_{q}} \subset A_{B_{q+1}}$ et $\quad A_{B_{q}} \subset A_{\alpha_{q+1}}$

$\left.2^{\circ}\right)$ Supposons maintenant que pour un entier $r, 1 \leqslant r \leqslant p-1$, on ait $A^{r}=A^{r+1}$. Alors pour tout $i \in I, A_{i}^{r}=A_{i}^{r+1}$. Donc pour tous $i, j$ dans $I, i \neq j$,

$$
\varphi\left(A_{i}^{r}\right)=\varphi\left(A_{j}^{r}\right)=U
$$

$U$ est un sous-espace ayant les $A_{i}^{r}$ pour bases et $U \supset S_{0}$. Comme $S$ est fini, $v=$ UnS est fini et :

$$
|v|=k \cdot r(v)+\left|s_{0}\right|>k \cdot r(v)
$$

ce qui contredit le fait que $S$ vérifie $\left(P_{k}^{\prime}\right)$

Donc on a bien une suite strictement croissante.

\section{Conclusion :}

Comme $S$ est fini et vérifie $\left(P_{k}\right)$, la propriété $\left(a_{p}\right)$ ne peut être vraie pour tout entier $p \geq 0$.

\section{Donc il existe un entier $p \geq 0$ tel que :}

$$
\text { et } \begin{cases}\left(a_{q}\right) & \text { est vraie pour } q=0,1, \ldots, p-1 \\ \left(a_{p}\right) & n ' \text { 'est pas vraie. }\end{cases}
$$

Construction de $\left(Y_{i}\right) \quad$ ( $L$ 'on suppose $p \geq 1$ )

Comme $\left(a_{p}\right) \cdot n$ 'est pas vérifiée,

$$
\begin{gathered}
\exists \alpha=(i(1), \ldots, i(p)) \in I_{p} \text { et } \exists i(p+1) \in I \quad \text { tels que : } \\
A_{\alpha} \notin \varphi\left(x^{i(p+1)}\right)
\end{gathered}
$$

Alors :

$$
\begin{array}{lll}
\forall x^{p} \in A_{\alpha}-\varphi\left(x^{i(p+1)}\right), & x^{p} \in x^{i(p)} \\
\forall x^{p-1} \in A_{\alpha(p-1)} \quad \text { tel que } & x^{p} \in x^{i(p)}\left(x^{p-1}\right) \\
\forall x^{k} \in A_{\alpha(k)} \quad \text { tel que } & x^{k+1} \in x^{i(k+1)}\left(x^{k}\right) \\
\text { pour } k=p-1, p-2, \ldots, 0 &
\end{array}
$$


on obtient une suite $\left(x^{0}, x^{1}, \ldots, x^{p}\right)$ telle que pour $k=1, \ldots, p$, $x^{k} \in x^{i(k)} \quad$ et $\quad x_{0}^{0} \in S_{0}$

Considérons maintenant le processus d'échanges successifs dans les $x^{i}$ où on fait passer $x^{p}$ de $x^{i(p)}$ dans $x^{i(p+1)}$. D'où :

$$
\begin{aligned}
T_{p}^{i(p)} & =x^{i(p)}-x^{p}, T_{p}^{i(p+1)}=x^{i(p+1)}+x^{p} \\
\text { et } \quad T_{p}^{i} & =x^{i} \quad \text { pour } i \in I-\{i(p), i(p+1)\}
\end{aligned}
$$

Puis par récurrence descendante, pour $k=p, p-1, \ldots, 1$

$$
\begin{aligned}
T_{k}^{i(k)} & =T_{k+1}^{i(k)}-x^{k}, T_{k}^{i(k+1)}=T_{k+1}^{i(k+1)}+x^{k} \\
\text { et } \quad T_{p}^{i} & =x^{i} \quad \text { pour } i \in I-\{i(k), i(k+1)\}
\end{aligned}
$$

Et finalement :

$$
T_{0}^{i(1)}=T_{1}^{i(1)}+x^{0} \text { et } T_{0}^{i}=T_{1}^{i} \text { pour } i \in I-i(1)
$$

est telle que $\left(\mathrm{Y}^{i}\right)=\left(\mathrm{T}_{0}^{i}\right) \quad$ est solution

En effet on vérifie par récurrence descendante sur $k$ que :

pour $k=p, p-1, \ldots, 0$ :

$$
\forall x \in A^{k^{\prime}-1} \quad, \quad T_{k}^{i(q)}(x)=x^{i(q)}(x)
$$

(utiliser le théorème 1 et les $\varphi_{\mathrm{A}} \mathrm{k}$ )

et en particulier pour $k=\mathrm{p}, \mathrm{p}-1, \ldots, 1$

$$
x^{k} \in A^{k}-A^{k-1}
$$

Donc les $\mathbf{r}_{\mathbf{k}}^{\mathbf{j}} \quad$ sont bien des parties libres

$$
\text { C.Q.F.D. }
$$

Remarque 1.- Quand $S$ vérifie $\left(P_{k}\right)$, la suite $A^{1} \subset \ldots \subset A^{q} \subset \ldots$ est strictement croissante tant qu'on peut la définir, et, au fur et à mesure de la construction, elle ne peut que s'allonger, un élément $x$ de $A^{q}$ ne pouvant passer que à $A^{q+k}$ où $k>0$. 
Remarque sur cet algorithme fini.

Soit $S$ une partie finie vérifiant $\left(P_{k}\right)$ où $k$ entier $\geq 2$. Quand on utilise l'algorithme indiqué, on construit sans permutation :

$$
\begin{array}{lll}
\mathrm{X}^{1} & \varphi \text {-base de } & \varphi(\mathrm{S}) \text { incluse dans } \mathrm{S} \\
\mathrm{x}^{2} & \varphi \text {-base de } & \varphi\left(S-\mathrm{X}^{1}\right) \text { incluse dans } S-\mathrm{X}^{1}
\end{array}
$$

$x^{p} \quad \varphi$-base de $\varphi\left(S-\underline{U}_{i=1}^{p-1} x^{i}\right)$ incluse dans $S-\underline{U}_{i=1}^{p-1} x^{i}$

pour $p=1,2, \ldots, k$

On obtient ainsi sans permutation $: x=\frac{\mathrm{U}}{\mathrm{p} I} \mathrm{x}^{\mathrm{p}} \subset \mathrm{S}$

Si on a de la chance, $X=S$ et l'algorithme n'est pas nécessaire; mais en général $X \varsubsetneqq S$. Cependant $X$ est une fraction assez grande de $S$ que nous allons essayer de minorer. En effet d'après $\left(\mathbb{P}_{k}\right)$ :

$$
k\left|x^{1}\right| \geq|s| \quad \text { et de manière cénérale, }
$$

pour $p \in I$,

$$
k\left|x^{p}\right|=k \cdot r\left(s-\underline{u}_{i=1}^{p-1} x^{i}\right) \geq|s|-\sum_{i=1}^{p-1}\left|x^{i}\right|
$$

Soit alors pour tout $p \in I,\left|x^{p}\right|=b_{p}|s|: b_{p}$ est un rationnel ayant $|s|$ comme dénominateur, et $0<b_{p} \leqslant 1$. Comme $|x|=|s|\left(b_{1}+\ldots+b_{k}\right) ;$ il nous suffit de minorer $b_{1}+b_{2}+\ldots+b_{k}$.

(1) s'écrit : $b_{1} \geq 1 / k, \ldots, b_{p} \geq 1 / k \quad\left(1-\sum_{i=1}^{p-1} b_{i}\right), \ldots$ donc

$$
b_{2} \geqslant 1 / k(1-1 / k), \quad b_{3} \geqslant 1 / k\left(1-1 / k^{2}, \ldots\right.
$$

et on démontre aisément nar récurrence sur $p=1, \ldots, k$ que

$$
b_{p} \geq 1 / k\left(1-1 / k^{n}\right)^{n-1}
$$


donc

$$
b_{1}+b_{2}+\ldots+b_{k} \geq 1-\left(1-1 / k^{k}\right.
$$

or la fonction $(1-1 / x)^{x}$ croit quand $x$ augmente $(x \geq 2)$ et quand $x$ tend vers $+\infty$, elle tend vers $1 / \ell$, d'où le résultat :

l'algorithme indiqué permet d'obtenir sans permutations

$$
x=U x^{i} \quad[i \in I] \text { telle que : }
$$

$$
|x| \geq\left(1-\left(1-1 / k^{k}\right)|s|\right.
$$

Si $k=2$, on obtient ainsi au moins les 7/8-ième de $S$, quand $k$ augmente, on en a une fraction qui va en diminuant, mais toujours supérieure a A $-1 / \ell \geq 0,63$.

Cas où $S$ est infini.

Soit $S$ une partie infinie de $E, S$ vérifiant $\left(P_{k}\right), \forall T \in \mathscr{T}(s)$, l'ensemble $P L(T)$ des partitions $\left(T_{i}\right)_{i \in I}$ de $T$ en $k$ parties libres est non vide $(I=\{1, \ldots, k\})$ et soient $T \subset U \in \mathcal{J}(S) .\left(U_{i}\right) \in \operatorname{PL}(U) \rightarrow\left(U_{i} \cap T\right) \in P L(T)$ est une application $f_{T U}$ de $\operatorname{PL}(U)$ dans $P L(T)$, le système (PL(T), $\left.f_{T U}\right)[T \in \mathcal{F}(S)]$ est un système projectif d'ensembles, et il nous suffit de démontrer que :

$$
\mathrm{PL}(\mathrm{S})=\lim \text { proj. }\left(\mathrm{PL}(\mathrm{T}), \mathrm{f}_{\mathrm{TU}}\right) \quad\left[\mathrm{T} \in \mathscr{F}^{(}(\mathrm{S})\right] \neq \emptyset .
$$

Nous avons déjà rencontré au chapitre $v$ un problème analogue: La démonstration se fait de la même manière ainsi :

On se ramène à une limite projective surjective en sorte que :

$$
\mathrm{PL}(\mathrm{S})=\lim \operatorname{proj}\left(\mathrm{QL}(\mathrm{T}), \mathrm{g}_{\mathrm{TU}}\right) \quad\left[\mathrm{T} \in \mathcal{F}^{\mathrm{F}}(\mathrm{S})\right]
$$

où $Q L(T) \subset P L(T)$ et $g_{T U}$ est la restriction de $f_{T U}$ à $Q L(T)$ en procédant de la même manière :

$$
\forall \mathrm{T}, \quad \mathrm{PL}^{1}(\mathrm{~T})=\cap \mathrm{f}_{\mathrm{TU}} \quad(\mathrm{PL}(\mathrm{U})) \quad[\mathrm{U} \in \mathcal{F}(\mathrm{S}), \quad \mathrm{U}>\mathrm{T}] \neq \varnothing
$$

d'où un système projectif $\left(\mathrm{PL}^{1}(\mathrm{~T}), \mathrm{f}^{1} \mathrm{TU}\right)$ où $\mathrm{f}^{1} \mathrm{TU}$ est la restriction de $\mathrm{f}_{\mathrm{TU}}$. 
Puis $\mathrm{PL}^{2}(\mathrm{~T}), \ldots$ et $\mathrm{QL}(\mathrm{T})=\cap \mathrm{PL}^{\mathrm{n}}(\mathrm{T}) \quad[\mathrm{n} \geq 1] \neq \emptyset$

Soit $\mathcal{P}_{\text {l'ensemble des familles }}\left(\mathrm{x}^{\mathrm{i}}\right)_{i \in I}$ de parties de $S$ telles que : $\forall \mathrm{T} \in \mathcal{F}(\mathrm{s}), \quad\left(\mathrm{x}^{\mathrm{i} \cap \mathrm{T})}, \mathrm{i} \in \mathrm{I} \in \mathrm{QL}(\mathrm{T}) \quad\right.$ (alors les $\mathrm{x}^{i}$ sont libres et $\left.U S_{i}[i \in I] \subset S\right)$. $\mathcal{S}$ ordonné par inclusion $\left(\left(X^{i}\right) \subset\left(Y^{i}\right)\right)$ est inductif, et (théorème de zorn) tout élément maximal $\left(M^{i}\right)_{i \in I}$ de $\mathcal{S}$ est tel que

$u M^{i} \quad[i \in I]=s$, i. e. $\left(M^{i}\right) \in \mathrm{PL}(s)$.

En effet si $M=U M^{i} \varsubsetneqq S$, soit $x \in S-M$. Pour tout $U \in J^{\prime}(M)$, l'ensemble $I_{U}$ des $i \in I$ tels que $U \cap S_{i}+x$ soit libre $n^{\prime} e s t$ pas vide, car $g_{U, U+x}$ est surjective. Donc $I_{x}=\cap I_{U}\left[U \in J^{\prime}(M)\right] \neq \varnothing \quad$ (car si $I_{x}=\varnothing$, on aurait $\varnothing=I_{U_{1}} \cap I_{U_{2}} \cap \ldots \cap I_{U_{n}}=I_{U_{1} \cup U_{2}} \ldots \cup U_{n}$, et $\forall i \in I_{x}$, $M^{i}+x$ est libre, et $\left(M^{i}\right)$ n'est donc pas maximale. 
Chap. VII Changements de bases. Applications aux matrices

Les théorèmes d'échange classiques montrent l'existence de bases ayant certaines propriétés, mais ne se préoccupent pas de la manière dont on peut les obtenir. Le but de cette section est d'étudier comment on peut construire de proche en proche une application $u$ d'une base $L$ dans une base $L^{\prime}$ en sorte que, par échanges successifs on ait toujours des bases. La première difficulté consiste à bien formuler la notion d'échanges successifs.

\section{1.- Lemmes et remarques préliminaires}

Soient $X$ et $L$ deux parties d'un ensemble $E, \leq$ un bon ordre sur $X$ et $u$ une application de $X$ dans $L$. Le procédé d'échanges successifs entre $X$ et $L$ défini par < et $u$ se conduit par récurrence transfinie dans $X$ ainsi :

- $\alpha$ étant le premier élément de $x, \quad L^{\alpha}=L$

- si $x$ a un prédécesseur $y, \quad L^{x}=L^{y}-u(y)+y$

- si $x$ n'a pas de prédécesseur, $L^{X}$ est l'ensemble des $z \in X \cup L$ pour lesquels il existe $y>x$ tel que pour tout $t \in] y, x\left[, \quad y \in L^{t}\right.$.

$L^{X}$ est bien défini par ces conditions car pour tout $z$ il n'y a qu'un nombre fini (trois au plus) de passages (soustraction ou addition) effectifs. En effet :

1) Si $z \in X-L, z \in L^{t}$ pour tout $t>z$ ( $z$ est ajouté une fois pour toute)

2) Si $z \in I-X$, ou bien $u^{-1}(z)=\emptyset$ et $z \in I^{t}$ pour tout $t$ ou bien $u^{-1}(z)=\varnothing$ et $x^{0}$ étant le plus petit élément de $\left.\left.u^{-1}(z), \forall t \in\right] \leftarrow, x^{0}\right], z \in L^{t}$ et $\left.\forall t \in\right] x^{0}, \rightarrow\left[, z \notin L^{t}\right.$

(Il se peut que $\left|u^{-1}(z)\right|>1$, globalement on échange alors $z$ et $u^{-1}(z)$, mais $l$ 'échange "successif" n'est "effectif" que pour $\left.x^{0}\right)$

3) Si $z \in X \cap L$, on note $x^{0}$ le plus petit élément de $u^{-1}(z)$ s'il existe. 3a) Si $u^{-1}(z)=\varnothing$, alors $z \in L^{t}$ pour tout $t$

3b) Si $u^{-1}(z) \neq \varnothing$, et $\left.\left.x^{0}>z, \forall t \in\right] \leftarrow, x^{0}\right], z \in L^{t}$ et $\left.\forall t \in\right] x^{0}, \rightarrow\left[, z \notin L^{t}\right.$

3c) Si $u^{-1}(z) \neq \varnothing, x^{0}<z$ et $\left.u^{-1}(z) c\right] \leftarrow, z[$, alors :

$\left.\left.\left.\left.\forall t \in] \leftarrow, x^{0}\right], z \in I^{t} ; \forall t \in\right] x^{0}, z\right], z \notin I^{t} ; \forall t \in\right] z, \rightarrow\left[, z \in L^{t}\right.$ 
3d) Si $u^{-1}(z) \neq \varnothing, \quad x^{0}<z, u(z) \neq z$ et $\left.u(z) \notin\right] \leftarrow, z\left[\right.$, en notant $x^{1}$ le plus petit élément $x$ de $u^{-1}(z)$ tel que $x>z$, on $a$ :

$\left.\left.\left.\left.\left.\forall t \in J \leftarrow, x^{0}\right], \quad z \in L^{t} ; \forall t \in\right] x^{0}, z\right], z \notin L^{t} ; \forall t \in\right] z, x^{1}\right], z \in L^{t} ;$ $\forall t \in] x^{1}, \rightarrow\left[, z \notin \mathrm{L}^{\mathrm{t}}\right.$.

Il reste à étudier les cas où $z \in u^{-1}(z)$, i.e. tels que $u(z)=z$ : on note alors $x^{\circ}$ le plus petit élément de $u^{-1}(z)$ et $x^{1}$ le plus petit $x \in u^{-1}(z)$ tel que $x>z$ s'il existe .

3a') $u^{-1}(z)=\{z\}: z \in L^{t}$ pour tout $t$

3b') $x^{0}=z$ et $x^{1}>z$ : alors $\forall t \leqslant x^{1}, z \in L^{t}$ et $\forall t>x^{1}, z \notin L^{t}$

$\left.3 c^{\prime}\right) x^{0}<z$ et $z$ est le plus grand élément de $u^{-1}(z)\left(x^{1}\right.$ n'existe pas) :

$\left.\left.\left.\left.\forall t \in] \rightarrow, x^{0}\right], z \in L^{t} ; \forall t \in\right] x^{0}, z\right], z \notin L^{t} ; \forall t \in\right] z, \rightarrow\left[, z \in L^{t}\right.$

3d') $x^{0}<z<x^{1}$ : alors

$\left.\left.\left.\left.\left.\forall t \in J \leftarrow, x^{0}\right\}, z \in L^{t} ; \forall t \in\right] x^{0}, z\right], z \notin L^{t} ; \forall t \in\right], z, x^{1}\right\}, z \in L^{t} ;$

$\forall t \in] x^{1}, \rightarrow\left[\quad, z \notin L^{t}\right.$

On constate un très grand arbitraire dans cette construction; mais comme on veut obtenir en fin de compte une partie $L^{X}$ contenant $X$, en ajoutant un plus grand élément $x^{\omega}$ à $X, L^{X}=L^{x \omega}$ si $X$ n'a pas de plus grand élément et $L^{X}=L^{X \omega}=L^{X}-u(x)+x$ si $X$ a $x$ pour plus grand élément, en particulier $L^{X}$ contient $X \cap L$; donc les cas $3 b), 3 d), 3 b^{\prime}$ ) et $3 d^{\prime}$ ) ne peuvent se produire. Les cas restant sont caractérisés par :

$$
\left.\forall z \in X \cap L, \quad u^{-1}(z) \subset J \leftarrow, z\right]
$$

En outre il est naturel d'exiger que l'échange soit "êffectif", i.e.

- ou bien $u(x) \in L^{x}$ et $x \notin L^{x}-u(x)$

- ou bien $u(x) \notin L^{x}$ et $x \in L^{x}-L^{x}-u(x)$

Alors nécessairement dans les cas 2) et $3 a) u^{-1}(z)$ a au plus un élément; dans le cas $3 c) u^{-1}(z)=\left\{x^{0}\right\}$; dans le cas $\left.3 a^{\prime}\right) u^{-1}(z)=z$; et le cas $\left.3 c^{\prime}\right)$ doit être écarté car $z \in \mathrm{L}^{2}$, donc $\mathrm{L}^{2+} \supsetneqq \mathrm{L}^{2}$. On constate alors aisément que :

Un prodécé d'échanges successifs tel que $L^{X} \supset X$ et tel que 1 'échange soit toujours effectif est caractérisé par le fait que : u est une injection et i $z \in X \cap I, \quad u^{-1}(z) \leqslant z$

Fn outre dans ce cas, en examinant chacun des cinq cas 1), 2), 3a), 3c) et $3 a^{1}$ ), on vérifie que : 
$\forall z \in X \cup L, \forall t \in X+X^{\omega}, z \in I^{t}$ si et seulement si $z \in M^{t}$

où $u^{t}=L-u\left(x_{t}\right)+x_{t}$ où $\left.x_{t}=\right] \leftarrow, t[$

Donc $\forall t \in X+x^{\omega}, I^{t}=u^{t}=L-u\left(x_{t}\right)+x_{t}$

Leme 1.- Soient $X$ et $L$ deux parties libres de $(E, \varphi)$ telles que $X \subset \varphi(L)$, $u$ une applijcation de $X$ dans $L, \leq$ un bon ordre sur $X$ et $\left(L^{X}\right)_{x \in X+x^{w}}$ associé au procédé d'échanges successifs $(u, \leq)$ de $X$ dans $L$. Alors $L^{X}=L^{X \omega}$ contient $X$ si et seulement si :

$$
\left.\left.\forall z \in X \cap L, \quad u^{-1}(z) \in\right]_{\leftarrow}, z\right]
$$

In outre les propriétés suivantes sont équivalentes :

a) $(u, \leq)$ vérifie (Ech) et $\forall x \in X+x^{\omega}, L^{x}$ est une base de $\varphi(L)$

b) $(u, \leq)$ vérifie (Ech') : $\forall x \in X \cap L, u(x) \notin u\left(x_{x}\right)$ où $\left.x_{x}=\right] \rightarrow, x[$; et $\forall x \in x+x^{\omega}, u^{x}=L-u\left(x_{x}\right)+x_{x}$ est une base de $\varphi(L)$

Si l'une de ces propriétés est vérifiée, u est une injection telle que : $\forall z \in I \cap L, u^{-1}(z) \leqslant z$ et pour tout $x \in X+x^{\omega}, \quad L^{x}=L-u\left(x_{x}\right)+x_{x}$

a) $\Longrightarrow\left(b, u\right.$ injectif et $L^{x}=x^{x}$ pour tout $\left.x\right)$

Dn raisonnant dans le $\varphi_{L^{x}-u(x)}$-espace $E$, on constate que l'échange est effectif pour tout $x \in X$, et corme $u$ vérifie (Ech), $u$ est une injection telle que : $\forall z \in X \cap L$, quand $u^{-1}(z) \neq \emptyset, \quad u^{-1}(z) \leq z$, et $\forall x \in X+x, L^{x}=u^{x}$. D'où aisément b).

b) $\Longrightarrow$ (a, u injectif et $L^{x}=u^{x}$ pour tout $\left.x\right)$

Il suffit de démontrer que : $u$ est une injection telle que : pour tout $z \in X \cap L, \quad u^{-1}(z) \leq z$ quand $u^{-1}(z) \neq \varnothing$.

$u$ est injective si et seulement si pour tout $x \in X, u(x) \notin u\left(x_{x}\right)$. Donc si u n'est pas injective, soit $x \in X$ le plus petit $y \in X$ tel que $u(y) \in u\left(x_{y}\right)$. Alors $x_{x} \neq \varnothing$, la restriction de $u \& x_{x}$ est injective et $u(x) \in u\left(x_{x}\right)$. Or $y^{x t}=L-u\left(x_{x}+x\right)+x_{x}+x$ et $M^{x}=L-u\left(x_{x}\right)+x_{x}$ sont deux bases de $\varphi(L)$, et $u^{x+}=u^{x}+x$, donc $x \in u^{x}: x \in X \cap L$, ce qui contredit (Ech').

Pour tout $z \in X \cap L$ tel que $u^{-1}(z) \neq \varnothing, u^{-1}(z) \leq z$. Sinon soit $x$ le plus petit $z \in X \cap L$ tel que $u^{-1}(z) \neq \emptyset$ et $u^{-1}(z)>z$. La restriction $u^{x}$ de $u$ a $x_{x}$ 
défini un procédé d'échanges successifs $\left(u^{x}, \leq\right.$ sur $\left.x_{x}\right)$ de $x_{x}$ dans $L$ tel que $x_{x} \subset L$ et (Ech). Donc $I^{t}=M^{t}$ pour tout $t<x$.

Comme $u(x)=x^{1} \notin x+x$ (sinon, $u^{-1}(x)=x$ ou $x^{1} \in X \cap L$ et $u^{-1}\left(x^{1}\right)=$ $x>x^{1}$, et $x$ ne serait pas minimal), $M^{x+}=M^{x}-u(x)+x$. Or si $x \in M^{x}$ (i.e. $\left.x \notin u\left(x_{x}\right)\right), u(x) \notin M^{x}$, donc $u(x) \in u\left(x_{x}\right)$, ce qui contredit (Ech'). De plus si $x \notin M^{x}, \quad x \in u\left(x_{x}\right) ;$ il existe donc $y_{1}<x$ tel que $u\left(y_{1}\right)=x$, mais $u\left(x_{1}\right)=x$, ce qui dontredit $u$ injective.

$$
\text { C.Q.F.D. }
$$

Remarque 1.- Si u est injective et vérifie (Ech), u vérifie (Ech'); mais en général, quand $u n^{\prime}$ 'est pas injective, même si (Ech) est vérifiée, (Ech') ne l'est pas. Inversement, il est aisé de construire u injective (donc vérifiant (Ech')), mais ne vérifiant pas (Ech), et a fortiori u non injective. Les conditions (Ech) et (Ech') sont donc indépendantes.

Remarque 2.- La condition (Ech') s'introduit naturellement pour $\left(M^{x}\right)$. En effet si $\left(M^{X}\right)$ est une base de $\varphi(L)$ pour tout $x \in X+x^{\omega}$

- ou $x \in X-I$ et $u(x) \notin u\left(x_{x}\right) \cup x_{x}$

- ou $x \in X \cap L$ et $x \notin M$ et $u(x) \notin u\left(x_{x}\right) \cup x_{x}$

- ou $x \in X \cap L$ et $x \in M^{X}$, alors

ou bien $u(x) \in M^{x}$, donc $u(x)=x$

ou bien $u(x) \notin M^{x}$, alors on peut choisir arbitrairement $u(x)$ dans $L-M^{x}$, i.e. dans $u\left(x_{x}\right)-x_{x} \cdot(E c h ')$ écarte donc cet arbitraire.

Remarque 3.- Si $(u, \leq)$ vérifie a) ou $b), u^{-1}(z) \neq \varnothing$ pour tout $z \in X \cap L$, car ou bien $u^{-1}(z) \cap x_{z}=\varnothing$ et $u(z)=z$, donc $u^{-1}(z)=z$, ou bien $u^{-1}(z)<z$.

\section{2.- Changements de bases.}

Définition 1.- $X$ et $L$ étant deux parties libres d'un $\varphi$-espace $E$ telles que $X \subset \varphi(L)$, on appelle changement de base (en abrégé : $c d b$ ) de source $X$, de but dans $L$, tout terme $(u, \leq)$ formé d'une application $u$ de $X$ dans $L$ et d'un ordre $\underline{\text { total }} \leqslant$ sur $X$ tels que : $u$ soit une injection de $X$ dans $L$ vérifiant $\forall x \in X, L-u\left(X_{x}\right)+X_{x}$ est une base de $\varphi(L)$ où $\left.x_{x}=\right] \leftarrow, x[$ et $\quad L-u(X)+X$ est une base de $\varphi(L)$. 
Théorème 1.- Soient $X$ et $L$ deux parties libres de $(E, \varphi)$ telles que $X \in \varphi(I)$, $\leq$ un bon ordre sur $X$ et $u$ une application de $X$ dans L. Alors les propriétés suivantes sont équivalentes :

1) $(u, \leq)$ est un $c d b$ de source $x$, de but dans $L$

2) $\forall x \in X+x^{\omega}, L^{x}$ est une base de $\varphi(L)$

et $\left.\forall x \in X \cap L, u^{-1}(x) \subset \quad J \leftarrow, x\right]$

3) $\forall x \in X+x^{\omega}, L-u\left(x_{x}\right)+x_{x}$ est une base de $\varphi(L)$

et $\forall x \in X \cap L, u(x) \& u\left(x_{x}\right)$

et alors, pour tout $x \in X \cap L, u^{-1}(x) \neq \varnothing$ et $u^{-1}(x) \leq x$;

et $\forall x \in X+x^{\omega} ; L^{x}=L-u\left(x_{x}\right)+x_{x}$

Justification de la terminologie : changements de bases.

Soit $(u, \leq)$ un $c d b$ de $X$ dans $L$. Alors $B=L$ et $B^{\prime}=L-u(X)+X$ sont deux bases de $\varphi(L)$, et u se prolonge trivialement, a savoir $v(x)=x$ pour tout $x \in L-u(x)$, en un $c d b \quad\left(v, s^{\prime}\right)$ de $B$ sur $B^{\prime}$ pour tout bon ordre $s^{\prime}$ sur $B$ induisant celui de $X$.

$\checkmark$ permet alors essentiellement de construire de proche en proche la base B' à partir de $B$, en n'ayant par échanges successifs que des bases intermédiaires; mais seule la restriction de $\mathrm{v}$ à $X$, $i . e . ~ u$, fait réellement des échanges, en sorte qu'il est techniquement préférable de raisonner sur $u$.

L'on note $\mathscr{C} \nless$ l'ensemble des $\mathrm{c} d \mathrm{~b}$ de $(E, \varphi) . \mathscr{C} \nless$ est ordonné par la relation : $(u, \leq)$ (resp. $\left(u^{\prime}, \leq^{\prime}\right)$ ) étant un $c d b$ de $X$ dans $L$ (resp. de $X '$ dans $\left.L^{\prime}\right)$, on dit que $(u, \leq)\left(u^{\prime}, s^{\prime}\right)$ et on lit u est restriction de $u^{\prime}$, ou u' prolonge $u$, si et seulement si :

$X \in X^{\prime}, L \in L^{\prime}, \leqslant$ induit $\leqslant$ sur $X$

et $u$ est la restriction de $u^{\prime} \grave{d} X$

Si $(u, \leq)$ est un $c d b$ de $x$ dans $L$, tout $\left(v, \leq^{\prime}\right)$ restriction de $(u, \leq)$ est aussi un $c d b$. Inversement :

Lemme 2.- Tout c d b $(u, \leq)$ de $X$ dans $I$ tel que $X$ ne soit pas une base de $\varphi(L)$ se prolonge en un $c d b \quad\left(v, s^{\prime}\right)$ de $X+x$ dans $L$ où $: x$ est un élément quelconque de $\varphi(L)-\varphi(X)$ donné à priori et $\leqslant$ ' le bon ordre sur $X+X$ prolongeant $\leqslant$ et pour lequel $x$ est le plus grand élément. 
En effet comme $L^{X}=L-u\left(X_{x}\right)+X_{x}$ est une base de $\varphi(L)$, il suffit de prendre $v(x) \in L^{x}$ tel que $v(x) \geqslant x$ si $x \in X \cap L$. Or :

si $x \in \mathrm{L}^{\mathrm{x}}$, on choisit $v(x)=x$

si $x \notin L^{x}$, comme $x+x$ est une partie libre, $L^{x}{ }_{x} \notin X^{\prime}{ }_{x}$ et on choisit $v(x) \in \mathrm{L}^{\mathrm{x}}{ }_{\mathrm{x}}-\mathrm{X}^{\prime}{ }_{\mathrm{x}}$

\section{3.- Propriétés des $\mathrm{c} \mathrm{d} \mathrm{b}$}

Le principal intérêt des $\mathrm{c} d \mathrm{~b}$ est qu'on peut en démontrer l'existence dans un grand nombre de cas. En effet le leme 2 permet de prolonger un peu tout c $d$ b de $X$ dans $L$ quand $\varphi(X) \neq \varphi(L)$; mais nous allons voir que $C$ n'est pas un ensemble inductif, en sorte que l'existence et la construction de $c \mathrm{~d} b$ pour les bons ordres infinis est délicate. Cette étude semble bien augurer de l'existence de $\mathrm{c} d \mathrm{~b}$ pour tout bon ordre sur $\mathrm{x}$. Voici une notion plus faible.

Soient $X$ et $L$ deux parties libres telles que $X \subset \varphi(L), \leq$ un bon ordre sur

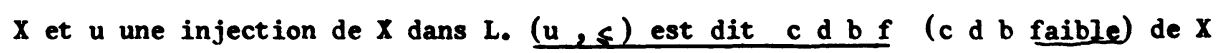
dans L si :

$\forall x \in X$, la restriction de $u$ à $X^{\prime}{ }_{x}$ est un $c d b$ de $x^{\prime}{ }_{x}$ dans $L$.

Soient $\leqslant$ un bon ordre sur $X$, u une application de $X$ dans $L .(u, \leq)$ est un $\mathrm{c} d \mathrm{~b} f$ de $X$ dans $L$ si et seulement si on a les propriétés :

(1) $X$ et $L$ sont des parties libres telles que $X \subset \varphi(L)$

(2) $\forall x \in X, I-u\left(X_{x}\right)+X_{x}$ et $L-u\left(X^{\prime} x_{x}\right)+X^{\prime}{ }_{x}$ sont des bases de $\varphi(L)$.

(3) $L-u(X)+X$ est une partie libre de $\varphi(L)$.

Donc un $c d b$ f $(u, \leq)$ est un $c d b$ si et seulement si $L-u(X)+X$-engendre $\varphi(L)$ (c'est toujours le cas si $(X, S)$ a un plus grand élément).

Leme 3.- Soit $(u, s)$ un $c d$ b $f$ de $X$ dans $L$ et a le premier élément de $X$. Si $L_{a}$ a deux éléments au moins, i.e. si :

$$
\begin{aligned}
L_{x} & =\left\{e_{0}, e_{1}, \ldots, e_{q}\right\} \text { où } q \geq 1 \\
\text { et où } a & =a_{0}=u^{-1}\left(e_{0}\right)<a_{1}=u^{-1}\left(e_{1}\right)<\ldots<a_{q}=u^{-1}\left(e_{q}\right)
\end{aligned}
$$


Alors, notant W I'application de $X$ dans $I$ définie par :

$W\left(a_{1}\right)=e_{0}, W\left(a_{0}\right)=e_{1}$ et $W(x)=u(x)$ pour les autres,

$(W, \leq)$ est un $c d b$ de $X$ dans $L$.

Ceci est faux en général si on échange les valeurs de $a_{i}$ et $a_{j}$ où $(i, j) \neq\{0,1\}$

\section{Démonstration.}

(1) et (3) de la proposition 1 sont vérifiées. Il suffit donc de démontrer que :

$$
\forall x \in X, L-W\left(X_{x}\right)+X_{x} \text { et } L-W\left(X^{\prime}{ }_{x}\right)+X^{\prime} x_{x} \text { sont des bases de } \varphi(L) \text {, }
$$

i.e. que :

$$
\forall x \in X, \quad a_{0}<x \leq a_{1}, N^{x}=L-W\left(x_{x}\right)+x_{x} \text { est une base de } \varphi(L) \text {. }
$$

or $\forall x \in X, \quad a_{0}<x \leq a_{1}, L^{X}=L-u\left(X_{x}\right)+x_{x}$ est une base de $\varphi(L)$, donc $N^{X}=L^{X}-e_{1}+e_{0}$ est une $\varphi$-base de $\varphi(L)$ si et seulement si $e_{0} \phi \varphi\left(L^{X}-e_{1}\right)$. Or si $e_{0} \in \varphi\left(L^{x}-e_{1}\right)$, comme $L_{a}+a-\left\{e_{0}, e_{q}\right\} \subset L^{x}-e_{1}$,

$$
\mathbf{L}_{\mathbf{a}}+\mathbf{a}-\mathbf{e}_{1} \subset \varphi\left(\mathbf{L}^{\mathbf{X}}-\mathbf{e}_{1}\right)
$$

donc $e_{\uparrow} \in \varphi\left(L^{x}-e_{1}\right)$, ce qui contredit $L^{x}$ libre.

L'on sait que en général les "valeurs" de $a_{i}$ et $a_{j}$ ne peuvent etre permutées dans les autres cas (considérer les matrices inversibles).

Lemme 4.- Soit $(u, \leq)$ un $c d$ b f de $X$ dans $L$ et $B$ une $\varphi_{L-u(X)+X^{-b a s e} \text { de }}$ $\varphi(L)$ incluse dans $u(X)-X$. Si $u n^{\prime}$ est pas $c d b, b \neq \varnothing$ et pour $b \in B$, soit $u^{-1}(b)=x_{0}$, il existe une suite $\left(x_{n}\right)_{0 \leqslant n<w}$ strictement croissante d'éléments de $x$ telle que :

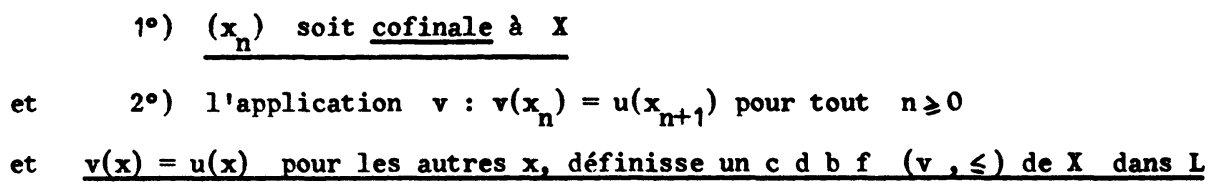

a) $\forall x \in X, \exists y \in X, y \geqslant x$ tel que $v(x)=u(y)$

et b) $\quad L-v(X)+X=(L-u(X)+X)+b$ démonstration. Posons $u_{0}=u_{\text {. }}$ En raisonnant dans le $\varphi_{X_{x_{0}}}$-espace $E$, et en uti-
lisant le lemme 3 , on déduit que : 
$\exists\left(x_{1}>x_{0}\right.$ tel que l'application $u_{1}$ :

$u_{1}\left(x_{0}\right)=u\left(x_{1}\right), u_{1}\left(x_{1}\right)=u\left(x_{0}\right)$ et $u_{1}(x)=u(x)$ pour les autres ( $x_{1}$ est unique si on le choisit successeur de $x_{0}$ dans $\left.u^{-1}\left(L_{x_{0}}-x_{x_{0}}\right)\right)$, définisse $\left(u_{1}, \leqslant\right)$ $c d$ b f de $X$ dans $L$.

On itère avec $x_{1}$, d'où $u_{2}$ et $x_{2}$ à partir de $u_{1}$ et $x_{1}$, puis avec $\left[x_{2}, u_{2}\right] \ldots$. On construit ainsi de proche en proche une suite $\left(x_{n}\right)_{n \geq 0}$ strie tement croissante dans $X$ telle que, pour tout entier $n \geqslant 1, l^{\prime a p p l i c a t i o n} u_{n}$ :

$$
\left\{\begin{array}{l}
u_{n}\left(x_{p}\right)=u\left(x_{p+1}\right) \text { pour } p=0,1, \ldots, n-1 \\
u_{n}\left(x_{n}\right)=b \text { et } u_{n}(x)=u(x) \text { pour les autres }
\end{array}\right.
$$

définisse un $c d$ b $f\left(u_{n}, \leqslant\right)$ de $X$ dans $L$.

Pour tout $n \geqslant 0$, la restriction $w_{n}$ de $u_{n}$ à $x_{x_{n}}$ définit un c $d$ b de $x_{x_{n}}$ dans L. On a ainsi une suite croissante de $c d b \quad\left(w_{n}, \leqslant\right)$, donc :

$$
\begin{aligned}
& W=\bigcup_{n} w_{n} \text { est unc d b f de } x^{\prime}=\bigcup_{n \geqslant 0} x_{x_{n}} \text { dans } L . \\
& \text { Si }\left(x_{n}\right)_{n \geqslant 0)} \quad n \text { 'est pas cofinale à } x \text {, soit } x=\operatorname{Sup~} x_{n}[0 \leqslant n<\omega] .
\end{aligned}
$$

On a $\mathrm{X}_{\mathbf{X}}=\mathrm{X}^{\prime}$ et $\mathrm{L}-\mathrm{W}\left(\mathrm{X}_{\mathbf{X}}\right)+\mathrm{X}_{\mathbf{X}}$ est $\varphi$-libre. Or $L-u\left(\mathrm{X}_{\mathbf{X}}\right)+\mathrm{X}_{\mathbf{X}}$ est une $\varphi$-base de $\varphi(L)$, d'où la contradiction puisque

$$
\begin{aligned}
& L-w\left(x_{x}\right)+x_{x} \supsetneqq \quad L-u\left(x_{x}\right)+x_{x} \\
& \left.\left.\left.D^{\prime} \text { où } 1^{\circ}\right) \text { et } 2^{\circ}\right), \quad \text { a) et } b\right), \text { et le : }
\end{aligned}
$$

Théorème 2.- L'ensemble des $c \mathrm{~d} b$ sur $(E, \varphi)$ muni de la relation d'ordre de prolongement $n$ 'est pas inductif. Plus précisément soit $\left(u_{i}, \leqslant_{i}\right)_{i \in I}$ une famille bien ordonnée de $c d b$ de $x_{i}$ dans $L_{i}$. Alors si le caractère final* de I n'est pas dénombrable,

$\left(u=\bigcup_{i} u_{i}, \leqslant=U_{i} \leqslant i_{i}\right)$ est unc d b de $x=\bigcup_{i} x_{i}$ dans $L=U L_{i}$; mais si le caractère final de $I$ est dénombrable, alors $(u, \leq)$ est un $c d$ b $f$ de $X$ dans $\mathrm{L}$ qui, en général, $n$ 'est pas un $c \mathrm{~d} b$.

*) voir A. Tarski, quelques théorèmes sur les alephs, Fund. Math. Vol. 7 (1929) p. 2 
Théorème 3.- Soient $X$ et $L$ deux parties libres telles que $X \subset \varphi(L)$. Alors pour tout bon ordre $\leq$ sur $X$ tel que :

$$
\alpha=\operatorname{Ord}(x, \leqslant)<\omega_{1}
$$

il existe une injection $u$ au moins de $X$ dans $L$ telle que $(u,<)$ soit un $c d b$ de $X$ dans $L$.

Corollaire.- Soient $X$ et $I$ deux parties libres telles que $X \subset \varphi(L), \leqslant$ un bon ordre sur $X$ et $Y$ un segment de $X$. Alors, si ord $(X-Y)<\omega_{1}$ on peut prolonger tout $c d b \quad(u, \leqslant Y)$ de $Y$ dans $L$ en un $c d b \quad\left(u^{\prime}, \leqslant\right)$ de $X$ dans $L$ d'au moins une manière.

Il suffit de raisonner dans $E$ considéré conme $\varphi_{\mathbf{Y}}$-espace.

Démonstration du théorème 3.

L'on va raisonner par récurrence transfinie dénombrable sur $\alpha$. Supposons le démontré pour tout ordinal $\lambda<\alpha$. Si $\alpha$ a un prédécesseur, il suffit d'appliquer le lemme 2. Sinon il existe une suite strictement croissante $\left(\alpha_{n}\right) 0 \leqslant n<\omega$ d'ordinaux $\alpha_{n}<\alpha$ telle que :

$$
\alpha=\operatorname{Sup} \alpha_{\mathrm{n}} \quad[0 \leqslant n<\omega]
$$

Soient $L^{\prime}$ une $\varphi_{X}$-base de $\varphi(L)$ incluse dans $L, Y=L-L^{\prime}:$ on a $L^{\prime}=I-Y$. Soit en outre $\leqslant$ un bon ordre sur $X$ tel que Ord $(X, \leqslant)=\alpha$.

Alors si

$X_{0}$ est le segment de $X$ isomorphe à $\alpha_{0}$

...

$x_{n}$ est le segment de $x-\bigcup_{p<n} x_{p}$ isomprphe à $\alpha_{n}-\alpha_{n-1}$

$\left(x_{n}\right)_{0 \leqslant n<\omega}$ est une partition de $x$.

Raisonnons dans le $\varphi_{0}$-espace $E$ où $\varphi_{0}=\varphi_{L^{\prime}} \quad$ : il existe $u_{0} c \mathrm{~d}$ b de $X_{0}$ dans $Y_{\text {. }}$

..

Raisonnons dans le $\varphi_{n}$-espace $E$ où $\varphi_{n}=\varphi_{n-1} x_{n-1}$, il existe $u_{n} c d$ b de $X_{n}$ dans $Y-U u_{p}\left(X_{p}\right) \quad[0 \leqslant p<n]$.

Finalement $u=\oplus u_{n}$ est un $c d$ b fe $X$ dans $L$ tel que $u(X) \subset Y$. Donc $\mathrm{L}-\mathrm{u}(\mathrm{X})+\mathrm{X}$ est une partie libre contenant $\mathrm{L}-\mathrm{Y}+\mathrm{X}: \mathrm{c}^{\prime}$ est une base de $\varphi(\mathrm{L})$.

$$
\text { C. Q. F. D. }
$$




\section{4.- Application aux matrices.}

Soit $A=\left(a_{i}^{j}\right) \quad[(i, j) \in I \times J] \quad$ une matrice sur un corps $k$, i.e. une application de $I \times J$ dans $k$ telle que :

$$
\forall j \in J, A^{j}=\left(a_{i}^{j}\right) \quad[i \in I] \in k^{(I)}
$$

(ce sont les vecteurs lignes). On note $\left(e_{i}\right)_{i \in I}$ la base canonique de $k^{(I)}$ et on suppose ici $J \subset I$ systématiquement.

Posant $L=\left\{e_{i} / i \in I\right\}$ et $X=\left\{A^{j} / j \in J\right\}, I$ 'on sait que $M$ est régulière si et seulement si $X$ est libre (famille libre) ce que l'on suppose désormais.

Soit un bon ordre $\leqslant$ sur $J$, d'où

$J=O(\alpha)$ et $\quad X=\left(A^{\nu}\right) \quad 0 \leqslant><\alpha$

Toute injection $u$ de $X$ dans $L$ définit une injection $\sigma$ de $J$ dans $I$ par $u\left(A^{\nu}\right)=A^{\sigma(\nu)}$ et inversement.

Alors $(u, \leq)$ est un $c d b$ si et seulement si :

(1) $\forall \nu<O(\alpha), L-u\left(\left\{A^{\lambda} / \lambda<\nu\right\}\right)+\left\{A^{\lambda} / \lambda<\nu\right\}$

est une base de $k^{(I)}$ ainsi que $L-u(X)+x$.

Si $\vee$ n'a pas de prédécesseur, on a une condition "forte", mais si $\vee$ a un prédécesseur, soient $v_{-1}, v_{-2}, \ldots, v_{-p}$ les prédécesseurs successifs de $\nu$ (où $\nu_{-p}$ n'a plus de prédécesseur), alors $(-1)$ pour $\nu$ est équivalente à : $\operatorname{det}\left(a_{i}^{j}\right) \quad\left[\sigma^{-1}(i), j=\nu_{0}, \nu_{-1}, \ldots, \nu_{-p}\right] \neq 0$

Du théorème 3 on déduit alors

Proposition 1 : Soit $A=\left(a_{i}^{j}\right) \quad[(i, j) \in I \times J]$ où $J=\{0,1, \ldots, n, . f \in I$ une matrice régulière sur un corps $k$. Alors il existe au moins une permutation $\sigma$ des recteurs colonnes de $A$ (i.e. une injection $\sigma$ de $J$ dans $I$ ) telle que :

a) Pour tout entier $p \geqslant 0$, notant $J_{p}=\{0,1, \ldots p\}$, on a

$$
\operatorname{det}\left(a_{\sigma(i)}^{j}\right) \quad\left[(i, j) \in J_{p} \times J_{p}\right] \neq 0
$$

et b) la matrice extraite $A_{0}=\left(a_{\sigma(i)}^{j} \quad[(i, j) \in J \times J]\right.$ soit inversible.

Quand $\mathrm{J}$ est fini, on retrouve un résultat classique. 
Remarque .- Soit A une matrice carrée d'ordre $n: I=J=\{1, \ldots, n\}$

La donnée d'un bon ordre $\leqslant$ sur $J$ est équivalente à celle d'une permutation $\sigma_{c}$ de $J$ et celle d'une innjection de $I$ dans $J$ à une permutation $\sigma_{p}$ de $I$. Alors $"\left(\sigma_{c}, \sigma_{\rho}\right) "$ est un c d b f pour tout couple de permutations si et seulement si tous les déterminants mineurs de $A$ sont non nuls, ce qui est en particulier le cas quand les $\left(a_{i}^{j}\right) \quad[(i, j) \in I \times J]$ sont algèbriquement libres sur le corps premier $k_{0}$.

\section{5.- Notions plus fortes}

Définition 2.- Soient $X$ et $L$ deux parties libres de $(E, \varphi)$ telles que $X \subset \varphi(L)$ et $u$ une injection de $X$ dans $L$. On dit que $u$ est un $c d b F$ (c $d$ b fort) de $X$ dans L si :

$\forall \mathrm{Y} \in \mathrm{X}, \mathrm{L}-\mathrm{u}(\mathrm{Y})+\mathrm{Y} \quad$ est une base de $\varphi(\mathrm{L})$.

Soient $X$ et $L$ deux parties libres de $(E, \varphi)$ telles que $X \subset \varphi(L)$ et $u$ une application de $X$ dans $L$. Les propriétés suivantes sont équivalentes :

(1) $u$ est un $c d$ b $F$ de $X$ dans $L$

(2) Pour tout ordre total $\leqslant \operatorname{sur} X,(u, \leq)$ est un $c d$ b de $X$ dans $L$.

(3) Pour tout bon ordre sur $x,(u, \leq)$ est un $c d b$ de $X$ dans $L$.

En général les $\mathrm{c} d \mathrm{~b}$ ne sont pas des $\mathrm{c} d \mathrm{~b} F$. On le constate aisément même pour $X$ ayant deux éléments.

La propriété pour u d'être un $c d$ b $F$ 'est pas de caractère fini en ce sens que si :

$\forall \mathrm{Y} \in \mathcal{f}(\mathrm{X}), \mathrm{u} / \mathrm{Y}$ est un $\mathrm{c} \mathrm{d} b \mathrm{~F}$ de $\mathrm{Y}$ dans $\mathrm{L}$,

Alors $u \quad n^{\prime}$ est pas en général un $c d$ b $F$ (ainsi considérer $L=\left(e_{n}\right)_{n} \geqslant 0$ base canonique de $\mathrm{K}^{(\mathrm{N})}$,

$$
x=\left\{x_{n} \mid x_{n}=e_{n+1}-e_{n} \text { et } n \geqslant 0\right\}
$$

et pour tout $\left.n \geq 0, u\left(x_{n}\right)=e_{n+1}\right)$.

Reprenons les notations du paragraphe 7.4 :

Une matrice $A=\left(a_{i}^{j}\right) \quad[(i, j) \in I \times J]$ est dite forte s'il existe une injection $\sigma$ de $J$ dans $I$ qui fait de $u$ un $c d b$ de $X$ dans $L$.

Il est aisé de vérifier la : 
Proposition 2.- Une matrice $A=\left(a_{i}^{j}\right) \quad[(i, j) \in I \times J]$, où $J$ est fini, est forte si et seulement si il existe une injection $\sigma$ de $J$ dans $I$ (d'où permutation des vecteurs colonnes) telle que tous les déterminants mineurs :

$$
\operatorname{det}\left(a_{\sigma(i)}^{j}\right) \quad\left[(i, j) \in K^{2}\right]
$$

où $K$ est une partie quelconque de $J$, sont non nuls.

Toutes les matrices régulières $2 \times 2$ sont fortes.

Les matrices régulières $3 \times 3$ non fortes sont toutes, à une permutation des lignes ou des colonnes près, et à une constante multiplicative non nulle près, de la forme :

$$
A=\left(\begin{array}{ccc}
1, & a, & 0 \\
b, & a b, & \lambda b \\
0, & a \mu, & \lambda \mu
\end{array}\right) \quad a b \lambda \mu \neq 0
$$

Ces matrices sont caractérisées par le fait que :

10) deux de ses éléments sont nuls

et $2^{\circ}$ ) deux de ses déterminants mineurs $2 \times 2$ sont nuls et deux seulement.

Démonstration : Si $\mathrm{A}$ est une matrice régulière $3 \times 3$ :

- on peut, par permutation des colonnes ou des linges, rendre les éléments de la diagonale principale non nuls.

- il y a au plus trois déterminants mineurs $2 \times 2$ nuls. Une matrice régulière $3 \times 3$ non forte est donc équivalente à :

$$
A^{\prime}=\left(\begin{array}{ccc}
1, & a & \lambda \\
b, & a b, & \mu \\
\lambda^{\prime} & \mu^{\prime} & c
\end{array}\right) \quad \begin{gathered}
a b c \neq 0 \\
(b \lambda-\mu)\left(\mu^{\prime}-a \lambda^{\prime}\right) \neq 0
\end{gathered}
$$

alors $A^{\prime}$ est faible si et seulement si :

$\lambda \mu=0, \lambda^{\prime} \mu^{\prime}=0$ et un second déterminant mineur $2 \times 2$ extrait de manière symétrique par rapport à la diagonale principale est nul.

\section{Matrices régulières $4 \times 4$ non fortes}

$1^{\circ)}$ Si une ligne ou une colonne a trois zéros, les matrices $4 \times 4$ régulières non fortes sont, à une permutation des lignes ou des colonnes près, et à une constante multiplicative non nulle près de la forme : 


$$
\left(\begin{array}{cccc}
c & 0 & 0 & 0 \\
a & 1 & a & 0 \\
\beta & b & a b & \lambda b \\
\gamma & 0 & a \mu & \lambda \mu
\end{array}\right) \quad \text { ou } \quad\left(\begin{array}{cccc}
c & \alpha & \beta & \gamma \\
0 & 1 & a & 0 \\
0 & b & a b & \lambda b \\
0 & 0 & a \mu & \lambda \mu
\end{array}\right)
$$

où $a$ b c $\lambda \mu \neq 0$

20) Sinon : on vérifie que toutes les matrices régulières $4 \times 4$ ayant huit éléments nuls sont fortes. Voici un exemple de matrice non forte :

$$
A=\left(\begin{array}{cccc}
a a^{\prime}, & 0, & a^{\prime}, & 0 \\
0 & b b^{\prime}, & b^{\prime}, & 0 \\
0, & 0, & c, & c c^{\prime} \\
a d, & b d^{\prime}, & 0 & -d c^{\prime}
\end{array}\right)
$$

où $a b c d a^{\prime} b^{\prime} c^{\prime} \neq 0$

$\left.3^{\circ}\right)$ Voici un exemple de matrice non forte n'ayant aucun coefficient nul :

$$
A=\left(\begin{array}{rrrr}
1 & 1 & 1 & 1 \\
1 & -1 & 1 & 1 \\
1 & 1 & -1 & -1 \\
1 & -1 & 1 & -1
\end{array}\right)
$$


Index terminologique

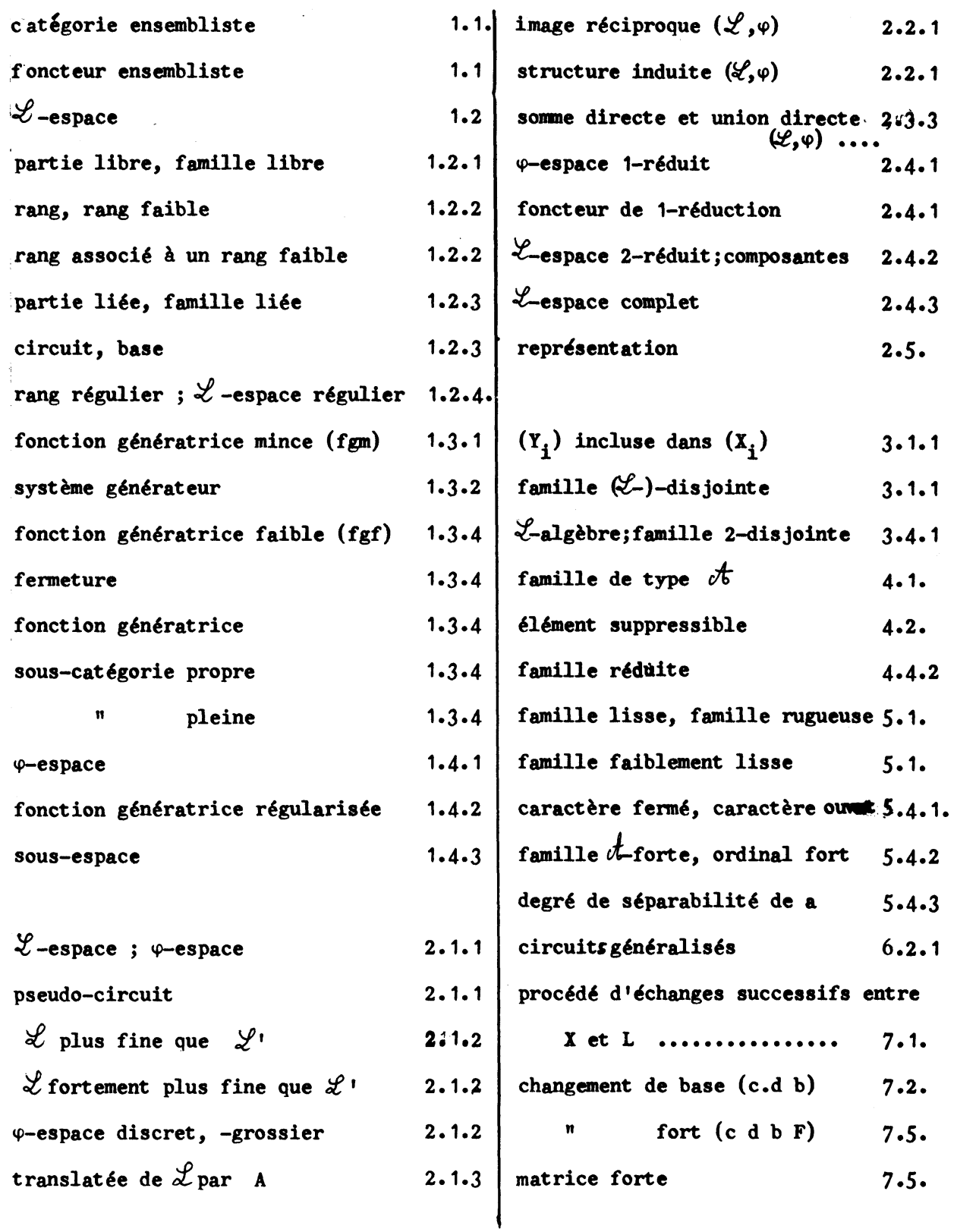




\section{Index des notations}

Les symboles, notations et terminologies de Bourbaki seront utilisées sans rappel, ainsi que les notations ensemblistes suivantes :

Notation utilisée

$\mathbf{X}+\mathbf{Y}$

$\mathbf{x}+\mathbf{x}$

$x-\mathbf{x}$

$x-x+y-z$
Notation Bourbakiste correspondante

$$
\begin{gathered}
X \cup Y \\
X \cup\{\mathbf{x}\} \\
X-\{x\} \\
(((X-\{x\})+\{y\})-\{z\})
\end{gathered}
$$

Elles seront utilisées quand aucune confusion ne sera à craindre avec des lois de composition.

En outre $\omega_{\alpha}$ : est l'ordinal initial d'indice $\alpha, U_{\alpha}$ est le cardinal aleph $\alpha, \omega_{\bar{\alpha}}$ le caractère final de $\omega_{\alpha}$ et si $a=U_{\alpha}, \bar{a}=U_{\bar{\alpha}}$ est le cardinal de finalité de $U_{\alpha^{*}}$ Tout ordinal $\nu$ est l'ensemble bien ordonné des ordinaux qui lui sont strictement inférieurs.

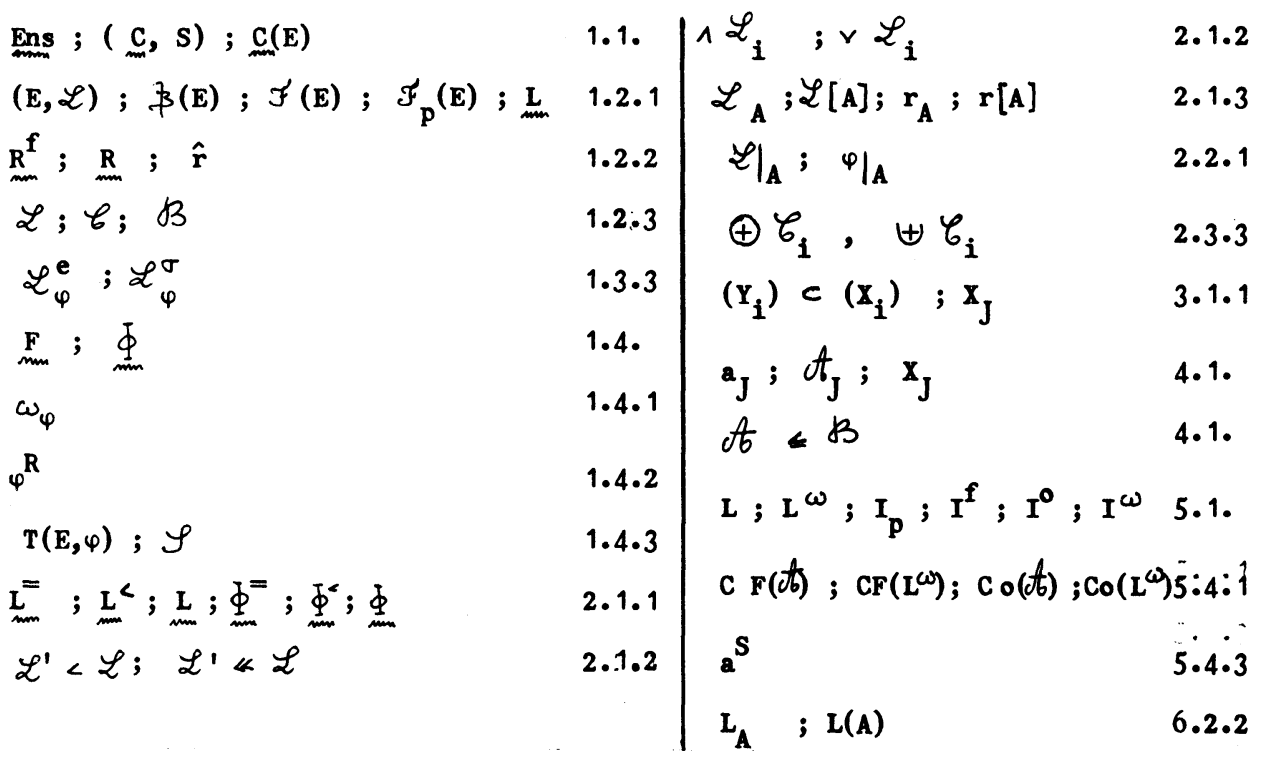




\section{BIBLIOGRAPHIE}

\section{Mémoires, notes et publications}

[1] HALL P., On representations of subsets, J. London Math. Soc., 10, 1934, p. 26

[2] HALMOS P.R. et VAUGHAN H.E., The marriage Problems, Amer. J. of Math., 72, 1950, p. 26.

[3] HAUPT 0., NOBELING et PAUC, 181, 1939, pp. 193-217. Uber abhangigkeitsraïme, J. für die reine und ang. Math,

[4] JONSSON B., Lattice-Theoretic approach to projective and affine geometry, Symposium on the Axiomatic Method, North-Holland publ. Comp., Amst erdam, 1959, p. 188.

[5] KONIG D. et VALKO S., über mehrdeutige Abbildungen von Mengen, Math.Annalen, 95, 1926, p. 135 .

[6] KUHN H.W., The Hungarian method for the assignement problem, Naval research quart erley, 2, 1955, p. 83 .

[7] MAC LANE S., A lattice formulation for transcendance degrees and p-bases, Duke Math. J., 4, 1938, pp. 455-468

[8] ORE 0., Graphs and matching theorems, Duke Math. J., 22, 1955, p. 625

[9] RADO R., A theorem on independence relations, Quaterley J. of Math. 13; 1942, pp. $83-89$

[10] RADO R., Axiomatic treatment of rank of infinite sets, Canadian J. of Math., 1, 1949, p. 337

[11] WHITNEY H., On the abstract properties of linear dependence, Amer. J. of Math., 57, 1935, p.p. 509-533.

[12] WHITNEY H., Non-separable and planar graphs, Trans. of the Amer. Math. Soc., 34, 1932, p. 339 .

[13] ROBERT P., Theorie générale de la disjonction, Comptes Rendus de l'Acad. des Sciences, 258, 1964, p.p.34-37. 
[14] ROBERT $P_{j}$, Sur un problème de disjonction (ensembliste, algébrique, etc.), Comptes Rendus de l'Acad. des Sciences, 258, 1964, p.p.6045-6048.

[15] TARSKI A., Quelques théorèmes sur les aleph, Fund. Math. Vol. 7 (1925)

[16] SAMUEL P., Modèles Boolèiens et Hypothèse du Continu (Résultats de P. Cohen par la méthode de P. Scott et R. Solovay), Séminaire Bourbaki, 1966/67. 317

[17] COHEN P., The independence of the continuum hypothesis, Proc. Nat. Acad. Sa1. USA, 50 (1963), 1143-1158 et 51 (1964), 105-110.

[18] ROBERT P., Méthodes fonctorielles sur l'axiomatique des systèmes générateurs, des rangs,...., Comptes Rendus de l'Acad. des Sciences, Paris, 260, 1965, pp. 4291-4294

[19] ROBERT P., idem, 260, 1965, pp. 4661-4664

[20] ROBERT P., idem, 260, 1965, pp. 5983-5986

[21] ROBERT P., Cours d'Algèbre, Faculté des Sciences de Caen, 1966-67.

[22] sMITH Jürgen, Eine verallgemeinerte Wohlordnung und die Endlichkeitsbedingungea der Wohlordnungstheorie, Arch, Math. 6 (1955) 374-381 


\section{Traités et Monographies}

[A] ARTIN E., Algèbre géométrique, Interscience Publ. New-York, 1957, chap.II, pp. 51-104

[B] BERGE C., Théorie des graphes et ses applications, 2e éd. Dunod, 1963

[C] BOURBAKI N., Eléments de Mathématiques. La référence $[\mathrm{C} ; \mathrm{v}, 4]$ indique le chapitre 4 du libre $v$ de la première partie. Il ne sera pas fait de références à la seconde partie. Acutalités Scientifiques et Industrielles, Hermann, Paris. Dernières éditions au 1-10-66.

[D] DENJOY A., L'énumération transfinie, Gauthier-Villars, Paris, 1946

[E] DUBREIL-JACOTIN M.L., LESIEUR L. et CROISOr R., Théorie des treillis, des structures algébriques ordonnées et des treillis géométriques, Gauthier-Villars, Paris, 1953.

[F] HALL M. $\mathrm{J}^{\mathbf{r}}$, The théory of groups, Macmillan $\mathrm{C}^{\circ}$, New-York, 1959.

[G] KONIG D., Theorie der endlichen und unendlichen Graphen, Leipzig, 1936 (Akàd. Verl. M.B.H.) et New-York, 1950 (Chelsea).

[H] SAMUEL P., MǦthọdes d'algèbre abstraite en géométrie algébrique, SpringerVerlag, Ergebnisse der Math., 1955.

[I] STEINITZ E., Algebraische Theorie der Körper, édité par R. Baer et H.Hasse, 1930.

[J] VAN DER WAERDEN, Modern algebra, 2e éd., Berlin, 1940

[K] WEIL A., Foundations of algebraic geometry, A.M.S., Colloq. publ.,XXXIX, 1962

[L] SAMUEL P. et ZARISKI 0., Commutative Algebra, Van Nostrand, 1958.

[M] GODEMENT R., Topologie algébrique et Théorie des Faisceaux, Hermann, A.S.I. 1252 , Paris 1958

[N] TSCHEBOTAROW et SCHWERDFEGER, Grundzüge der Galois'schen Theorie, Noordhoff, 1950, Groningen.

[0] BAER R., Linear algebra and Projective Geometry, Acad. Press. Inc. Pub., New-York, 1952

[P] BENABOU J., Structures algébriques dans les catégories, Thèse, 1966.

[Q] COHN P.M. Universal Algebra, Harper and Row, New-York, 1965.

[R] EHRESMANN C., Catégories et Structures, Dunod, Paris, 1965. 